Ciepielewska-Kaczmarek/ Jentges / Tammenga-Helmantel

\title{
Landeskunde im Kontext
}

Die Umsetzung von theoretischen Landeskundeansätzen in DaF-Lehrwerken

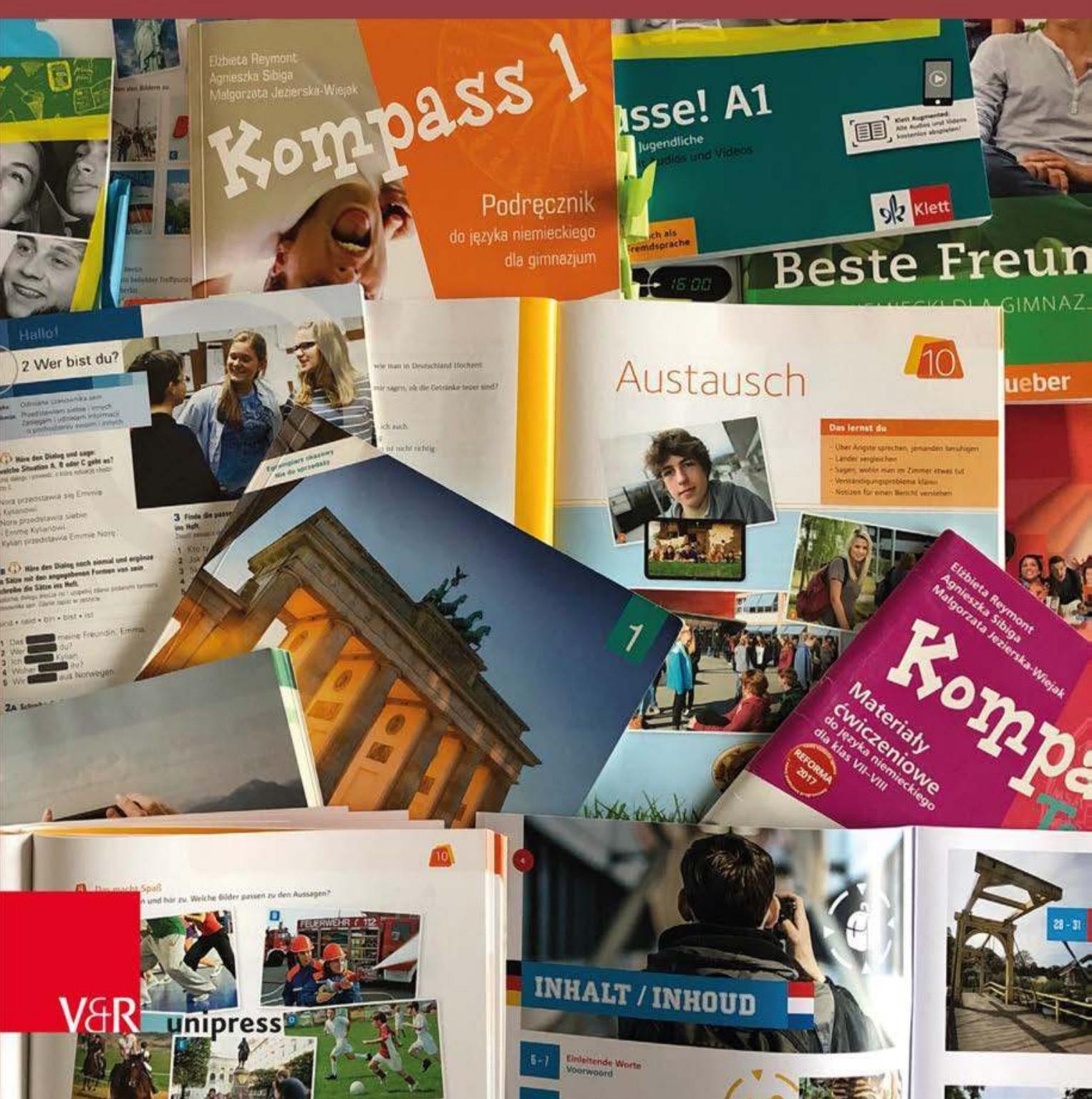




\section{V\&R unipress}

Open-Access-Publikation im Sinne der CC-Lizenz BY-NC-ND 4.0

(C) 2020, Vandenhoeck \& Ruprecht GmbH \& Co. KG, Göttingen

ISBN Print: 9783847111214 - ISBN E-Lib: 9783737011211 
Open-Access-Publikation im Sinne der CC-Lizenz BY-NC-ND 4.0

() 2020, Vandenhoeck \& Ruprecht GmbH \& Co. KG, Göttingen

ISBN Print: 9783847111214 - ISBN E-Lib: 9783737011211 
Luiza Ciepielewska-Kaczmarek / Sabine Jentges / Marjon Tammenga-Helmantel

\section{Landeskunde im Kontext}

Die Umsetzung von theoretischen Landeskundeansätzen in DaF-Lehrwerken

Mit 58 Abbildungen

V\&R unipress 
Bibliografische Information der Deutschen Nationalbibliothek

Die Deutsche Nationalbibliothek verzeichnet diese Publikation in der Deutschen Nationalbibliografie; detaillierte bibliografische Daten sind im Internet über https://dnb.de abrufbar.

Gedruckt mit freundlicher Unterstützung von Meesterchapsteam Vakdidactiek MVT und dem Institut für Angewandte Linguistik der Adam-Mickiewicz-Universität.

@ 2020, Vandenhoeck \& Ruprecht GmbH \& Co. KG, Theaterstraße 13, D-37073 Göttingen Dieses Werk ist als Open-Access-Publikation im Sinne der Creative-Commons-Lizenz BY-NC-ND International 4.0 (»Namensnennung - Nicht kommerziell - Keine Bearbeitungen«) unter dem DOI 10.14220/9783737011211 abzurufen. Um eine Kopie dieser Lizenz zu sehen, besuchen Sie https://creativecommons.org/licenses/by-nc-nd/4.0/. Jede Verwertung in anderen als den durch diese Lizenz zugelassenen Fällen bedarf der vorherigen schriftlichen Einwilligung des Verlages.

Umschlagabbildung: @ Luiza Ciepielewska-Kaczmarek

Vandenhoeck \& Ruprecht Verlage | www.vandenhoeck-ruprecht-verlage.com

ISBN 978-3-7370-1121-1 


\section{Inhalt}

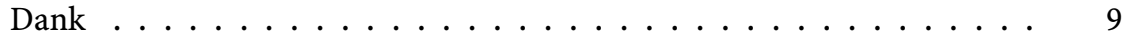

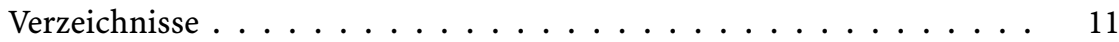

Abbildungsverzeichnis ................... . . . 11

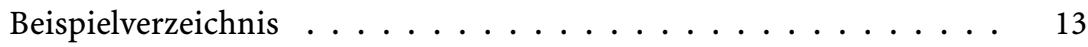

Abkürzungsverzeichnis . . . . . . . . . . . . . . 13

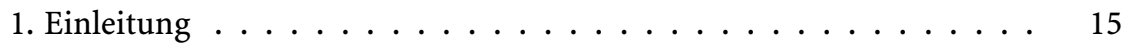

2. Rolle der Lehrwerke für den fremdsprachlichen Unterricht . . . . . 19

2.1 Vom Lehrbuch zum Lehrwerk . . . . . . . . . . . . . . . . . . . . . . 19

2.2 Lehrwerke heute . . . . . . . . . . . . . . . . 22

2.3 »Zankapfel Lehrbuch« - Diskussion über das Für und Wider der

Lehrwerkverwendung im FSU . . . . . . . . . . . . . . . . 25

2.4 Lehrwerkanalyse und Lehrwerkkritik . . . . . . . . . . . . . 28

3. Landeskundliche und kulturelle Inhalte im Fremdsprachenunterricht. 33

3.1 Landes- und Kulturkunde: Inhalte und Ansätze . . . . . . . . . 34

3.2 ABCD und DACH: Integration der Vielfalt . . . . . . . . . . . . . 37

3.3 Zielgruppen-Orientierung als Prinzip . . . . . . . . . . . . . 42

3.4 Weitere Ansätze: Integrative und Erlebte Landeskunde . . . . . . 45

3.4.1 Das Tübinger Modell einer integrativen Landeskunde . . . . 46

3.4 .2 Erlebte Landeskunde . . . . . . . . . . . . . . . . . . . . . . 50

3.5 Interkulturelles Lernen . . . . . . . . . . . . . . . . . . 52

3.5.1 Interkulturelles vs. kulturreflexives Lernen . . . . . . . . . . . . 58

3.5.2 Diskursive Landeskunde . . . . . . . . . . . . . . 59

3.5.3 Spatial turn: »Räume sagen mehr als tausend Worte» . . . 63

3.5.4 Kontaktdidaktik . . . . . . . . . . . . . 67

3.6 Ausblick: Landes- und Kulturkunde in DaF-Lehrwerken . . . . . . 71 
4. Lehrmaterial-Analysen . . . . . . . . . . . . . . . . . . . . . . . . . . . . . 75

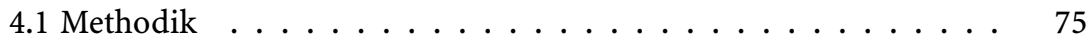

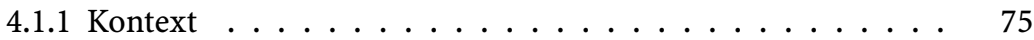

4.1.2 Analyseverfahren . . . . . . . . . . . . . . . 76

4.2 Landeskunde in niederländischen Lehrwerken . . . . . . . . . . . . . 78

4.2.1 Neue Kontakte. . . . . . . . . . . . . . . . . . 80

4.2.1.1 Stellung und Art der Vermittlung von Landeskunde

in Neue Kontakte . . . . . . . . . . . . . . . . 81

4.2.1.2 Landeskundeansätze und Prinzipien in Neue

Kontakte . . . . . . . . . . . 83

4.2 .2 Na klar! . . . . . . . . . . . . . . . . . 89

4.2.2.1 Stellung und Art der Vermittlung von Landeskunde in Na klar! . . . . . . . . . . . . . . . . . . . . 90

4.2.2.2 Landeskundeansätze und Prinzipien in Na klar! . . . 94

4.2 .3 TrabiTour . . . . . . . . . . . . . . . . . . 98

4.2.3.1 Stellung und Art der Vermittlung von Landeskunde in TrabiTour . . . . . . . . . . . . . . . . . 99

4.2.3.2 Landeskundeansätze und Prinzipien in TrabiTour . . 102

4.2.4 Fazit: Niederländische Lehrwerke . . . . . . . . . . . . 106

4.3 Landeskunde in polnischen Lehrwerken . . . . . . . . . . . 107

4.3 .1 Mit links! . . . . . . . . . . . . . . . . . . . . . . . . 109

4.3.1.1 Stellung und Art der Vermittlung der Landeskunde in Mit links! . . . . . . . . . . . . . . . . . . . . 110

4.3.1.2 Landeskundeansätze und Prinzipien in Mit links! . . 111

4.3 .2 Kompass Team . . . . . . . . . . . . . . . . . . 118

4.3.2.1 Stellung und Art der Vermittlung von Landeskunde in Kompass Team . . . . . . . . . . . . . . . . . . 119

4.3.2.2 Landeskundeansätze und Prinzipien in Kompass

Team . . . . . . . . . . . . . 120

4.3.3 Meine Deutschtour . . . . . . . . . . . . . . . . 123

4.3.3.1 Stellung und Art der Vermittlung von Landeskunde in Meine Deutschtour . . . . . . . . . . . . . . . . . . 124

4.3.3.2 Landeskundeansätze und Prinzipien in Meine Deutschtour . . . . . . . . . . . . . . 127

4.3.4 Fazit: Polnische Lehrwerke . . . . . . . . . . . . . . . 134

5. Good practice . . . . . . . . . . . . . . . . . . . . 137

5.1 Kulturelle und sprachliche Vielfalt des deutschen Sprachraums . . 138

5.2 Kontaktdidaktik: Verbindungen zwischen dem vermeintlich fremden und eigenen kulturellen und sprachlichen Raum entdecken . . . . . . . . . . . . . . . . . . 154 
5.3 Kulturreflexivität und Diskursivität . . . . . . . . . . . . . 165

6. Ausblick . . . . . . . . . . . . . . . . . . 175

Literaturverzeichnis . . . . . . . . . . . . . . . . . 179 
Open-Access-Publikation im Sinne der CC-Lizenz BY-NC-ND 4.0

() 2020, Vandenhoeck \& Ruprecht GmbH \& Co. KG, Göttingen

ISBN Print: 9783847111214 - ISBN E-Lib: 9783737011211 


\section{Dank}

Ermöglicht wurde die Fertigstellung dieser Monographie mit Hilfe von verschiedenen Kollegen und Kolleginnen sowie Institutionen, denen wir Dank schulden. Bedanken möchten wir uns bei Camilla Badstübner-Kizik, Minna Maijala, Stefanie Ramachers, Paul Sars und Gabriela Gorąca-Sawczyk für kritisches Feedback und inhaltliche Tipps und Hinweise. Unser Dank gilt auch Iris Schoonen und Loes Verswijveren, für Unterstützung bei Recherche und formalen Arbeiten, sowie Marko Gorupec für Hilfe bei digitalen Aufgaben. Dank sagen wir ebenfalls den Lehrwerksverlagen dafür, dass sie eine Abdruckgenehmigung für ihre Aufgabenmaterialien und Abbildungen erteilten. Für finanzielle Unterstützung bedanken wir uns ganz herzlich beim Meesterschapsteam Vakdidactiek MVT (dem niederlandeweiten Expertenteam für Fremdsprachendidaktik, https://modernevreemdetalen.vakdidactiekgw.nl), Frau Prof. Izabela Prokop, der Leiterin des Instituts für Angewandte Linguistik an der AdamMickiewicz-Universität (www.ils.amu.edu.pl) und dem CLS (Centre for Language Studies, https://www.ru.nl/cls/) der Radboud Universiteit Nijmegen. Dem Vandenhoeck \& Ruprecht Verlag, insbesondere Herrn Oliver Kätsch und Frau Julia Schwanke, danken wir für die Aufnahme des Bandes ins Verlagsprogramm und die hervorragende Kooperation.

Poznań, Nimwegen, Groningen, im Dezember 2019 Luiza Ciepielewska-Kaczmarek, Sabine Jentges, Marjon Tammenga-Helmantel 
Open-Access-Publikation im Sinne der CC-Lizenz BY-NC-ND 4.0

() 2020, Vandenhoeck \& Ruprecht GmbH \& Co. KG, Göttingen

ISBN Print: 9783847111214 - ISBN E-Lib: 9783737011211 


\section{Verzeichnisse}

\section{Abbildungsverzeichnis}

\begin{tabular}{|c|c|}
\hline Abb. 1 & Kaffeehäuser \\
\hline Abb. 2 & Man spricht Deutsch \\
\hline Abb. 3 & Wie soll das Kind denn heißen \\
\hline Abb. 4 & Wortbedeutung "privat« und »öffentlich» \\
\hline Abb. 5 & Wortbedeutung "Heimat" \\
\hline Abb. 6 & $\begin{array}{l}\text { Reflexive Auseinandersetzung mit } \\
\text { »remden«: Spione unter uns }\end{array}$ \\
\hline Abb. 7 & $\begin{array}{l}\text { Reflexive Auseinandersetzung mit } \\
\text { »Fremden«: Gegenseitige Wahrnehmung }\end{array}$ \\
\hline Abb. 8 & $\begin{array}{l}\text { Relativierung der »eigenen« Kultur: } \\
\text { Wertvolle Zeit }\end{array}$ \\
\hline Abb. 9 & $\begin{array}{l}\text { Relativierung der »eigenen« Kultur: } \\
\text { Typische Eigenschaften? }\end{array}$ \\
\hline Abb. 10 & Typisch Mann, typisch Frau? \\
\hline Abb. 11 & $\begin{array}{l}\text { Erkundung des eigenen Raums: } \\
\text { Sprachspurensuchen }\end{array}$ \\
\hline Abb. 12 & $\begin{array}{l}\text { Erkundung des eigenen Raums: } \\
\text { Fotochallenge Memory }\end{array}$ \\
\hline Abb. 13 & Kontaktdidaktik: Stadtführung \\
\hline Abb. 14 & Kontaktdidaktik: Tour durch die Schule \\
\hline Abb. 15 & $\begin{array}{l}\text { Der gymnasiale Bildungsweg in Polen, den } \\
\text { Niederlanden und Deutschland }\end{array}$ \\
\hline Abb. $16 a$ & Schwytzerdütsch \\
\hline Abb. $16 b$ & Schwytzerdütsch \\
\hline Abb. 17 & So quatscht Berlin \\
\hline Abb. $18 \mathrm{a}$ & Literatur - Das Wunder von Bern \\
\hline Abb. $18 b$ & Literatur - Ernst Jandl \\
\hline Abb. 19 & Die Sächsische Schweiz und Aussprache \\
\hline Abb. 20 & Deutsch auf Bildern \\
\hline Abb. 21 & Rätsel Kuchen \\
\hline Abb. 22 & Begrüßungsfloskeln in Europa \\
\hline
\end{tabular}

Bayerlein 2013: 54-55

Matecki 2017: 10-11

Hansen/Zuber 1996: 41

Behal-Thomsen et al. 1993: 45

Behal-Thomsen et al. 1993: 126

Hansen/Zuber 1996: 35

Lundquist-Mog 1996: 14

Hansen/Zuber 1996: 39

Lundquist-Mog 1996: 15

Altmayer 2016: 22

Immers 2017: 13

Immers 2017: 23

Immers 2017: 18

Immers 2017: 19

Tab.

Neue Kontakte 3/A: 175

Neue Kontakte 3/A: 176

Neue Kontakte 3/B: 99

Na klar 3A: 170

Na klar 3A: 171

Na klar 3B: 18

TrabiTour 1: 6

TrabiTour AB-A: 54

Mit links! KB 1: 6 
Abb. 23 Bezug zur Lebenswelt der Jugendlichen am Mit links! KB 1: 73

Beispiel des Themas Sport: Eine Recherche-

Aufgabe zu Stadien in Polen

Abb. 24 Stimulierung einer kulturreflexiven

Mit links! KB 1: 70

Haltung am Beispiel des Themas Feste

Abb. 25 Einführung der Jahreszahlen als Beispiel für Meine Deutschtour KB 8: 57

Sprach- und Landeskundeinhalte

integrierende Aufgabe

Abb. 26 Relevanz der deutschen Sprache im europäischen bzw. weltweiten Kontext

Abb. 27 Beispiel für Aufgaben zur Stimulierung

Meine Deutschtour KB 7: 14-15

einer kulturreflexiven Haltung am Beispiel

des Themas Schulbeginn

Abb. 28

Beispiel für Aufgaben zur Stimulierung

Meine Deutschtour KB 7: 141

einer kulturreflexiven Haltung am Beispiel

des Themas Schulsystem und

Schulnotensystem

Abb. 29 Häuser in Deutschland und in Polen

Meine Deutschtour KB 8: 139

Abb. 30 D-A-CH-L

Abb. 31 DACH-Länder: persönlicher Zugang

Abb. 32 Die deutsche Sprache in Deutschland,

Team Deutsch 3: 6-7

Team Deutsch 2: 6-7

Österreich und der Schweiz

Abb. 33

Sprachenpolitik

Pilaski et al.: 8

Abb. 34

Musik aus Liechtenstein

Abb. 35

Die deutschen Dialekte

Abb. 36a

DACH Memory-Karten

Abb. 36b

DACH Memory-Karten

Abb. 37

Typisch DACH: Klischees

Pilaski et al.: 14

Zugspitze TAB 4 vwo, 7-8

Deutsch echt einfach A1: 57

Jentges/Holtland: 11

Jentges/Holtland: 12

Team Deutsch 3: 10

Abb. 38 Was ist das? Rätselbilder

Dimensionen Magazin 2: 92-93

Abb. 39

Landeskunde: Borkum und Reit im Winkel

Abb. 40

Städte und Landschaften

Abb. 41

Das ist mein Land

Abb. 42

Blick aus dem Fenster

Klasse A1: 125

Abb. 43

Das ist unsere Stadt

Abb. 44

Das ist unser Lieblingsort

Abb. 45

Sprachen in meinem Land

Abb. 46a Sprachenportraits

Dimensionen Magazin 2: 16-17

Beste Freunde A1.1: 25

Prima Plus A1.2: 52

Beste Freunde A 1.2: 39

Beste Freunde A2.2: 21

Team Deutsch 3: 64

Abb. $46 \mathrm{~b}$

Sprachenportraits

Abb. 47

Obst in Deutschland

Dimensionen Magazin 3: 72

Dimensionen Magazin 3: 73

Abb. 48

Schilderwelten

Abb. 49

Karten, Karten, Karten

Abb. 50

Austauschberichte

Klasse A1: 65

Altmayer, Claus (Hg.) (2016): KV 8

Altmayer, Claus (Hg.) (2016): KV 50

Prima Plus A2.2: 27

Abb. 51

Sprachen im DACH-Alltag

Team Deutsch 3: 57

Abb. 52

Küß die Hand

Abb. 53

Wie geht's

Abb. 54

In meinem Kühlschrank

Hansen/Wendt 1996: 45

Hansen/Wendt 1996: 48

Dimensionen Magazin 3: 30-31 


\section{Beispielverzeichnis}

Beispiel 1 Das besondere Detail

Beispiel 2 Rätsel: Länder und Städte

Beispiel 3 Die deutschsprachigen Länder und Regionen

Beispiel 4 Musik

Beispiel 5 DACH-Memo-Spiel: Aufgabe

Beispiel 6 Städte und Landschaften

Beispiel 7 Warum lernt ihr Deutsch?

Beispiel 8 Video über eure Stadt

Beispiel 9 Werbeplakat

Beispiel 10 Souvenir, Souvenir; Touristenführung; Dokumentation

Beispiel 11 Die Buchkritiker

Beispiel 12 Die Schule international

Beispiel 13 Der fremde Blick von außen

\section{Abkürzungsverzeichnis}

$\begin{array}{ll}\text { NeKo } & \text { Neue Kontakte } \\ \text { NK } & \text { Na klar! } \\ \text { TT } & \text { TrabiTour } \\ \text { MD } & \text { Meine Deutschtour } \\ \text { ML } & \text { Mit links! } \\ \text { KT } & \text { Kompass Team } \\ \text { KB } & \text { Kursbuch } \\ \text { AB } & \text { Arbeitsbuch }\end{array}$


Open-Access-Publikation im Sinne der CC-Lizenz BY-NC-ND 4.0

() 2020, Vandenhoeck \& Ruprecht GmbH \& Co. KG, Göttingen

ISBN Print: 9783847111214 - ISBN E-Lib: 9783737011211 


\section{Einleitung}

Landeskundliche Inhalte und kulturelle oder interkulturelle bzw. kulturreflexive Aspekte dürften zweifelsohne als fester Bestandteil des modernen Fremdsprachenunterrichts zu bezeichnen sein. Doch wie sieht das in der Praxis aus? Welche didaktisch-methodischen Konzepte oder Ansätze stehen hinter der Vermittlung solcher Inhalte im Fremdsprachenunterricht? Welche Aufgabenoder Übungstypen bieten sich an, um bestimmte Lernziele im Bereich von Landeskunde, Kulturvermittlung, Interkulturalität und Kulturreflexivität im Unterricht umzusetzen? Unterscheiden sich diese in verschiedenen Ländern bzw. in den dort produzierten Lehrmaterialien?

Die fremdsprachige Unterrichtspraxis ist - neben curricularen Voraussetzungen - stark durch die jeweils zur Verfügung stehenden und ausgewählten Lehrwerke bzw. das jeweils eingesetzte Lehrwerk geprägt; der (curriculare) Kontext, in dem ein Lehrwerk erscheint, hat einen großen Einfluss auf die Inhalte und Schwerpunkte sowie die jeweils im Lehrwerk getroffenen didaktischen Entscheidungen. Dieser Band bietet eine Darstellung theoretischer Grundlagen zur Lehrwerk- bzw. Landeskundeforschung und erkundet dann deren konkrete Umsetzung in einer kontextualisierten Lehrwerkanalyse. Untersucht werden jeweils drei gegenwärtig frequent im schulischen Deutsch-als-FremdspracheUnterricht ( $\mathrm{DaF})$ eingesetzte Anfänger-Lehrwerke für Jugendliche, die für den niederländischen und den polnischen Markt produziert wurden. Eine Analyse von Lehrwerken aus eben diesen beiden Ländern (Polen und die Niederlande) ist insofern relevant und äußerst interessant, als dass es sich um Nachbarländer des deutschsprachigen Gebiets handelt; beide mit vergleichsweise hohen prozentualen Anteilen Deutsch lernender Schüler und Schülerinnen, jedoch mit jeweils deutlich unterschiedlichen kontextuellen und curricularen Rahmenbedingungen und Traditionen im Fremdsprachenunterricht.

Der vorliegende Band gliedert sich in sechs Kapitel. Anschließend an das einleitende erste Kapitel fokussiert Kapitel 2 auf die Bedeutung der Lehrwerke und der Lehrwerkanalysen für den fremdsprachlichen Unterricht. Thematisiert wird die Entwicklung vom Lehrbuch als Kursbuch mit Texten, Aufgaben und 
einer Grammatikübersicht zum heutigen Lehrwerk als Materialienpaket, das auch aus einer ganzen Reihe von Zusatzkomponenten mit unterschiedlicher didaktischer Funktion besteht. Ebenfalls werden Diskussionen um Lehrmaterial, wie Vor- und Nachteile von Lehrwerken und die Relevanz des Zielgruppenbezugs angesprochen. U.a. beschränkter Zielgruppenbezug hat dazu geführt, dass neben globalen Lehrwerken auch regionale Lehrwerke entstanden, die meist kontrastiv angelegt sind. Schließlich folgt eine Auseinandersetzung zur Geschichte der Lehrwerkforschung, die seit gut 40 Jahren als eines der zentralen Arbeitsgebiete in der Fremdsprachendidaktik gesehen wird und für die Unterrichtspraxis vor allem bei der Einschätzung, inwieweit ein Lehrwerk für eine bestimmte Lernergruppe ${ }^{1}$ und in einem bestimmten Unterrichtskontext geeignet ist, äußerst relevant ist.

Kapitel 3 bietet eine Übersicht der Geschichte der Landes- und Kulturkundeforschung und deren Entwicklung im Bereich der Fremdsprachendidaktik. Neben `traditionellen` Ansätzen der Landeskunde im fremdsprachlichen Unterricht werden weitere Entwicklungen präsentiert, wie die ABCD-Thesen und das DACH-Prinzip, die Einsichten aus der Integrativen und Erlebten Landeskunde und der Begriff des interkulturellen und kulturreflexiven Lernens. Auch neueste Entwicklungen wie etwa die Diskursive Landeskunde und der sogenannte spatial turn werden vorgestellt. Konkrete Beispiele aus Lehrwerken veranschaulichen die präsentierten Ansätze und Theorien.

Kapitel 4 ist der Landeskunde in Lehrwerken gewidmet. Die konkrete Umsetzung von landeskundlichen Inhalten in jeweils drei DaF-Lehrwerken aus Polen und den Niederlanden wird auf Basis der im vorherigen Kapitel vorgestellten, möglichen landes- und kulturkundlichen Konzepte und Ziele dahin gehend analysiert, wie sich diese in den einzelnen Lehrwerken widerspiegeln. Hierbei wird ausführlich auf den Unterrichtskontext von DaF in diesen beiden Ländern eingegangen. Der Aufbau der Analysen erfolgt wie folgt: Lehrwerkbeschreibung, die den Aufbau, die Thematik und die Position, die Landeskunde im Lehrwerk hat, zeigt; Darstellung, welche Art der Landeskundevermittlung das Lehrwerk bietet, ob diesbezüglich überwiegend explizit oder vielmehr implizit, additiv, isoliert bzw. integrativ vorgegangen wird; auch wird auf die Visualisierung und den Anschluss zur Lebenswelt der Zielgruppe von landeskundlichen Inhalten eingegangen.

Kapitel 5 stellt eine Sammlung von good practice-Beispielen dar. Aus einer Skala an internationalen DaF-Lehrwerken und Zusatzmaterialien, die den Blick von DaF- Experten erweitern können, die in der Regel gut im Bilde sind, was im eigenen Land an Lehrmaterialien vorliegt, werden in übersichtlicher Weise ge-

1 Zugunsten der Lesbarkeit sind im vorliegenden Band alle Personenbezeichnung generisch zu verstehen, es sind stets alle Geschlechter gemeint. 
lungene Beispiele für die Umsetzung unterschiedlicher landeskundlicher Inhalte und Lernziele präsentiert, so dass Anregungen für ein breiteres Spektrum an Aktivitäten vorgestellt werden, die direkt in die eigene Praxis umgesetzt werden können. Vorgestellt werden Aufgaben,

- die dem DACH-Prinzip entsprechend die kulturelle und sprachliche Vielfalt des deutschen Sprachraums zeigen und erfahren lassen,

- die nach den Einsichten der Kontaktdidaktik Lernenden die Möglichkeit bieten, Verbindungen zwischen dem vermeintlich fremden und eigenen kulturellen Raum zu entdecken,

- in denen angestrebt wird, eine kulturreflexive Haltung bei Lernenden $\mathrm{zu}$ fördern und es den Lernenden ermöglicht wird, am zielsprachigen Diskurs teilzunehmen,

- die Erlebte Landeskunde ermöglichen und Möglichkeiten eröffnen, wie Lernende die zielsprachige Kultur direkt und affektiv erfahren können.

Abgeschlossen wird der Band mit einer kurzen Zusammenfassung und einem Ausblick. Das Buch bietet sowohl erfahrenen sowie angehenden Lehrpersonen des Faches Deutsch als Fremdsprache (DaF) im In- und Ausland als auch Fachdidaktikern und Forschern an Universitäten und Hochschulen im DaFBereich eine reiche, verständliche und inspirierende Quelle für ihre tägliche (Unterrichts)praxis und die Ausbildung von Lehrpersonen. Geboten wird eine mehrperspektivische Übersicht zum Thema \Landes- und Kulturkunde` und deren Umsetzung in Lehrwerken, wobei das Angebot an Möglichkeiten für den Landes- und Kulturkundeunterricht aus Theorie und (Lehrwerk-)Praxis präsentiert, verglichen und kontextualisiert wird. Damit möchte der vorliegende Band Anregungen bieten, wie eine abwechslungsreiche, relevante und unterschiedliche Lernziele verfolgende Integration von landes- und kulturkundlichen Inhalten im Fremdsprachenunterricht erfolgen kann. 
Open-Access-Publikation im Sinne der CC-Lizenz BY-NC-ND 4.0

() 2020, Vandenhoeck \& Ruprecht GmbH \& Co. KG, Göttingen

ISBN Print: 9783847111214 - ISBN E-Lib: 9783737011211 


\section{Rolle der Lehrwerke für den fremdsprachlichen Unterricht}

Fremdsprachen gehören im institutionalisierten Lernen inzwischen zum Fächerkanon und die Alterseinstiegsgrenze rückt weltweit immer weiter nach unten. Der Lernmaterialienmarkt boomt schon seit mehreren Jahren, was unter anderem an der Zahl der jedes Jahr erscheinenden Neubearbeitungen und Neuveröffentlichungen im Bereich des Fremdsprachenlernens sichtbar wird. Die weltweit große Nachfrage nach Fremdsprachenkenntnissen ${ }^{2}$ ist vor allem eine der Folgen der Globalisierung im wirtschaftlichen wie im privaten Bereich. Die Internationalisierung des Arbeitsmarktes, berufliche und private Mobilität, neue Zielgruppen (wie beispielsweise Kleinkinder oder Senioren), Deutsch nach Englisch-Lernende erfordern stetig neue Veränderungen des Fremdsprachenlehr- und -lernangebots.

Im folgenden Kapitel wird versucht, die Rolle zu bestimmen, die gegenwärtig das Lehrwerk im institutionalisierten Unterrichtsprozess einnimmt. Unser Augenmerk gilt auch dem Stellenwert und der Funktion der Lehrwerkanalysen im Rahmen der Unterrichtsentwicklung und -optimierung.

\subsection{Vom Lehrbuch zum Lehrwerk}

Der Einsatz der Lernmaterialien im Sprachlernprozess hat eine lange Tradition. Der Begriff Lernmaterial umfasst nach Rösler und Schart (2016: 483) »Material zu bestimmten Themen oder sprachlichen Phänomenen, das unter didaktischen Gesichtspunkten produziert, ausgewählt oder adaptiert worden ist«. Mit Lernmaterialien sind einzelne Materialien wie Lehrwerke, Texte, Abbildungen, Spiele, Filme, Zeitungsartikel, Radiosendungen gemeint, die sowohl für den Unterricht als auch für ganz andere Zwecke verfasst wurden, und dann für das Sprachenlernen eingesetzt werden (vgl. Rösler/Würffel 2014: 12). Der Begriff

2 Es muss an der Stelle angemerkt werden, dass große Nachfrage an Fremdsprachenkenntnissen sich nicht per se in höheren Deutschlernerzahlen widerspiegelt. 
Lehrwerk referiert auf ein Materialienpaket, in dem die einzelnen Komponenten systematisch aufeinander bezogen sind und einen Medienverbund bilden (vgl. Krumm/Ohms-Duschenko 2001: 1029). Rösler und Schart (2016: 483) sprechen dem Lehrwerk die wichtige Rolle zu, »den Lernprozess für einen bestimmten Zeitraum zu begleiten oder zu steuern und die Vielfalt der sprachlichen und kulturellen Phänomene der Zielsprache zu behandeln und unter didaktischen Gesichtspunkten in Beziehung zu bringen«. Das Lehrwerk als ein »komplexes Medienverbundsystem " (Funk 2016: 441) verschiedenster Unterrichtsmaterialien, das wir heute so kennen, ist in der Geschichte des Fremdsprachenlernens relativ neu. Noch bis in die 1960er Jahre des 20. Jahrhunderts beanspruchte die überwiegende Zeit im fremdsprachlichen Unterricht die Arbeit mit dem Lehrbuch, einem in sich abgeschlossenen "Druckwerk mit fest umrissener didaktischer und methodischer Konzeption (Zielsetzung; Lernstoffprogression; Unterrichtsverfahren), in dem alle zum Lernen benötigten Hilfsmittel (Texte; Übungen; Grammatikdarstellung; Vokabular, etc.) rzwischen zwei Buchdeckeln enthalten sind (Neuner 2003: 399).

Die Loslösung vom Lehrbuch als unterrichtsdominierendem Medium ist einerseits auf den rasanten technologischen Fortschritt, andererseits auf die Entwicklung der fremdsprachlichen Lehrmethoden zurückzuführen. Die Dominanz von Lesen und Textarbeit in der Grammatik-Übersetzungsmethode, die zur Beherrschung der Sprache über die Kenntnis von Wörtern und grammatischen Regeln führte, korrespondierte noch mit dem Lehrbuchkonzept. Erst im Rahmen der audiolingualen und audiovisuellen Methode nahmen technische Hilfsmittel (Kassettenrecorder; Overhead-Projektor) und mit ihnen zusätzliche Arbeitsmaterialien einen bedeutenden Platz ein. Die Entwicklung der Kommunikativen Didaktik Mitte der 1970er Jahre und das neue Lernziel der kommunikativen Kompetenz brachten einen Wandel vom Lehrbuch- zum Lehrwerkkonzept. Die Zielsetzung in der kommunikativen Methode - die Ausbildung der sprachlichen Fertigkeiten des Lesens, des Schreibens, des Sprechens und des Hörverstehens - erforderte neue Bestandteile des Lehrwerks.

Ein Lehrwerk besteht von da an aus einer ganzen Reihe von Zusatzkomponenten mit unterschiedlicher didaktischer Funktion. Eine zentrale Stütze des institutionalisierten Fremdsprachenunterrichts stellt heute überwiegend das Kursbuch (auch Schülerbuch genannt) dar, das Texte enthält, in denen die Sprache in verschiedenen Situationen präsentiert wird. Storch (1999: 282) spricht in diesem Zusammenhang von Lehrwerken, die all das enthalten, was "zum Erlernen einer fremden Sprache als Kommunikationsmittel erforderlich ist: den Stoff, d.h. die in die Progression eingebundenen Texte und Übungen zur Einführung und Festigung von Wortschatz, Grammatik und Redemitteln, ebenso wie die Verstehens- und Äußerungsanlässe zur Förderung der kommunikativen Fertigkeiten«. Ergänzende, weiterführende und vertiefende Übun- 
gen und Aufgaben sowohl für den Unterricht als auch zum selbständigen Lernen zu Hause werden in einem Übungs- oder Arbeitsbuch angeboten. Kurs- und Arbeitsbuch können separat oder in einem Band vorliegen.

Allerdings liegen kulturelle Unterschiede im Bereich der Lehrwerksgestaltung vor (Harwood 2013). So bieten beispielsweise niederländische, schwedische und finnische DaF-Lehrwerke im Textbuch meist ausschließlich Texte, Vokabeln und eine Grammatikübersicht an. Das Arbeitsbuch, das im Unterricht und für Hausaufgaben bzw. Selbststudium leitend ist, enthält die Aufgaben und bietet Verweise zum Textbuch, das vielmehr als Nachschlagewerk funktioniert. In anderen Regionen, darunter auch in Polen, gilt, was für Lehrwerke, die im deutschsprachigen Raum für den weltweiten Einsatz produziert wurden, zutrifft: Das Kursbuch ist meist unterrichtssteuernd konzipiert und kann unabhängig vom Arbeitsbuch eingesetzt werden. Das Arbeitsbuch bietet vertiefende und wiederholende Übungsangebote, die zum Selbststudium bzw. als Hausaufgaben eingesetzt werden, oft auch optionale Extras.

Zum Medienverbund gehören heute außerdem CDs mit Audio-Dateien oder weiteren Lese- und Hörtexten, mit interaktiven Wortschatz- und/oder Grammatikübungen, DVDs mit Video-Dateien (u.a. mit Landeskundefilmen). Zu anderen, ergänzenden Komponenten gehören Glossare, Vokabel- und Grammatiktrainer, Lesehefte etc. Nahezu zu jedem Lehrwerk wird ein Lehrerhandbuch angeboten, das von einem (kommentierten) Lösungsschlüssel bis hin zu einer umfangreichen Handreichung mit kommentierten Unterrichtsvorschlägen, fertigen Kopiervorlagen für den Unterricht und Tests reichen. Viele Verlage stellen Lehrenden und Lernenden zu ihren Lehrwerken nicht nur einen digitalen Unterrichtsassistenten, sondern auch Onlineangebote wie Downloadmaterialien, Online-Tests, Zusatztexte oder weiterführende Links zur Verfügung. Diese können sowohl im Unterricht als auch zu Hause abgerufen und bearbeitet werden. Auch wird das multimediale Angebot immer häufiger mit augmented learning angereichert. Mit einer Augmented-App können die Dateien aus dem Kursbuch eingescannt und auf dem Smartphone oder Tablet abgespielt werden. Diese technologische Neuerung ist erstmals in den Lehrwerken großer Verlagshäuser implementiert worden.

Das Angebot der niederländischen Verlage geht da eindeutig weiter; alle Kursbücher stehen den Lernenden sowohl in Buchform als auch digital zur Verfügung. Dazu bieten die Verlage auf ihrer Online-Plattform u. a. adaptive Grammatik- und Wortschatztrainer, extra Anleitungsvideos, diagnostische Tests, Leseund Hörtexte zum Lehrwerk und Transkripte zu den Hörtexten.

Die Multimedialisierung der Lehr- und Lernmaterialien, also dass das »ursprünglich rein schriftliche Medium um eine Fülle auditiver, visueller und interaktiver Elemente zu einem multimedialen Verbund« (Thaler 2011: 22) erweitert wurde, ist zweifellos dem technischen Fortschritt zu verdanken. Durch 
diese Expansion, wie Thaler (2011: 21) die "zahlenmäßige Ausdehnung» der Lehrwerke auf zahlreiche Begleitmaterialien sowohl für Lehrer als auch für Lerner bezeichnet, erhofft man sich ein effektiveres Erreichen der jeweiligen Lernziele. Thaler (2011: 21) macht aber zugleich auf eine Gefahr der »Materialschwemme" aufmerksam, die diese "Aufblähung des Lehrwerks" mit sich bringen kann. Auch Grünewald (2017: 36) sieht es als eine Herausforderung für Lehrpersonen, die neuen Medien tatsächlich als eine Bereicherung und Erweiterung des bisherigen Medienrepertoires zu sehen und vor allem entsprechend im Unterricht einzusetzen.

Der Einsatz dieses breiten Angebotes in die alltägliche Unterrichtspraxis muss im jeweiligen länderspezifischen Kontext gesehen, d.h. diskutiert und ausgewählt werden (vgl. dazu Kap. 4).

\subsection{Lehrwerke heute}

Lehrwerke können seit gut 20 Jahren als ein Programm zur Entfaltung der Lehrund Lernarbeit, in dem allgemeine didaktische Prinzipien, methodische Kategorien und pädagogische Leitvorstellungen berücksichtigt werden müssen (vgl. Neuner 1995), gesehen werden. Die fremdsprachlichen Lehrwerke orientieren sich inzwischen nicht nur europa-, sondern bereits weltweit, an sechs Niveaustufen des Gemeinsamen europäischen Referenzrahmens (GER): A1, A2, B1, B2, C1, C2. Dabei gibt es heute auf dem Markt nur wenige Lehrwerke, die durchgängig von A1 zu C2 führen. Die meisten Lehrwerke sind so konzipiert, dass sie das Erreichen eines bestimmten Sprachniveaus anstreben. Das größte Angebot findet man bei Lehrwerken für die Niveaustufen A1 bis B1 und das sowohl für die Zielgruppe Kinder, Jugendliche als auch für erwachsene Lerner. Rösler (2012: 43) betont, dass wenn Lehrwerke den Anspruch haben, mehr als eine Sammlung von Lehrmaterial zu sein,

dann müssen sie einen inneren Aufbau haben, der den Lernenden den Erwerb erleichtert. Dazu gehört, dass Wortschatz, Grammatik, Aussprache, Landeskunde, Textsorten, Redeintentionen usw. nicht einfach nebeneinanderstehen, sondern in einer ästhetisch interessanten, unterhaltsamen und doch lehrreichen Weise aufeinander bezogen werden. Dies geschieht u. a. dadurch, dass das Lehrwerk in Lektionen aufgeteilt wird, die nach einem bestimmten Gesichtspunkt wie Handlungsort oder Thema ein Profil bekommen.

Dem Lehrwerk kommt viel mehr zu als nur die Aufgabe Texte zu liefern: Ein Lehrwerk soll »die Gesamtheit der Anforderungen an Spracharbeit in den Bereichen Aussprache, Lexik, Wortschatz, Grammatik, Textarbeit usw. abdecken und mit einer Übungs- und Aufgabenvielfalt verbinden« (Rösler/Schart 2016: 485). Ein gutes Lehrwerk sollte darüber hinaus Identifikationsmöglichkeiten 
bieten, denn wie Seliger (1977: 275, zitiert in: Butzkamm 1993:11) unterstreicht, »Being exposed to language is not like being exposed to a virus. One doesn't catch it automatically ... The learner must do something active that involves him cognitively in the process". Aus diesem Grund sieht Pfeiffer (2008: 60) universelle Lehrwerke kritisch und betont, dass nur ein Lehrwerk, das für konkrete Adressaten (Lernende wie Lehrende), konkrete Unterrichtsbedingungen und konkrete Unterrichtsziele entwickelt wurde, den Anspruch erheben kann, für diese ein optimales Werkzeug zu sein. Auch Maijala (2007: 557) betont die bedeutende Rolle, die ein klarer Bezug zur Zielgruppe bei der Erstellung von Lehrwerken einnimmt. Zielgruppenbezug umfasst u.a.

- den Bezug zum Alter der Lernenden - demnach unterscheidet man grob zwischen Lehrwerken für Kinder, Jugendliche und Erwachsene; es kann aber noch weiter differenziert werden, z. B. für junge Erwachsene, für Kinder im Primarbereich,

- den Bezug zum angestrebten Sprachniveau - demnach gibt es Lehrwerke, die für Anfänger konzipiert wurden, was nach dem GER den Niveaustufen A1, A2, B1 entspricht und Lehrwerke für die Mittelstufe als auch für fortgeschrittene Lernende - Niveaustufen B2, C1 und vereinzelt auch für C2,

- den Bezug zu Lernzielen, demnach kann es sich um allgemeinsprachliche Lehrwerke handeln als auch um fachsprachenbezogene wie z. B. Wissenschaftsdeutsch, Wirtschaftsdeutsch oder Deutsch für Mediziner. Es sind gegenwärtig auf dem Markt Lehrwerke erhältlich, die sowohl die Alltagssprache als auch die Fachsprache vermitteln wollen, als auch solche, deren Fokus auf Vermittlung von Fachsprache liegt.

In Bezug auf die Zielgruppe wird zwischen einsprachigen und kontrastiven Lehrwerken unterschieden, die des Weiteren in globale/überregionale, regionalisierte und regionale DaF-Lehrwerktypen unterteilt werden können. Überregionale Lehrwerke werden für den globalen Markt produziert. Es handelt sich hier vor allem um innerhalb des deutschsprachigen Raums produzierte einsprachige Lehrwerke. Was im Zusammenhang mit überregionalen, einsprachigen Lehrwerken nicht selten bemängelt wird, ist die Tatsache, dass sie für ein globales, nicht-spezifisches Publikum zusammengestellt werden (vgl. Maijala/ Tammenga-Helmantel 2016: 537) und dadurch weder auf die sprachlichen und kulturellen Kenntnisse der Lernenden noch ausreichend auf die bisher gemachten Sprachlernerfahrungen eingehen können (vgl. Rösler 2013: 216). Auch Tomlinson (2012: 158) sieht für das globale Publikum verfasste Lehrwerke kritisch: "Many global coursebooks are not considered to be sufficiently engaging or relevant for their actual users. In attempting to cater for all students at a particular age and level, global coursebooks often end up not meeting the needs and wants of any«. 
Bei der Verwendung globaler Lehrwerke können Missverständnisse entstehen, besonders dort, wo unterschiedliche Lehr- und Lerntraditionen aufeinandertreffen. Rösler und Würffel (2014: 88) sprechen von Unzufriedenheit, Abwehrhaltungen oder sogar von offenen Konflikten, wenn Unterrichtsmaterialien und -methoden unbedacht in einen bestimmten Kontext, in dem z.B. andere Lern-/Lehrtraditionen existieren, importiert werden. Wenn globale Lehrwerke zum Einsatz kommen, passiert es deshalb nicht selten, dass die Lehrpersonen vor der durchaus herausfordernden Aufgabe stehen, das Lehrwerk hinsichtlich der Lerntraditionen einer konkreten Lernergruppe vor Ort methodisch-didaktisch anzupassen (vgl. Maijala/Tammenga-Helmantel 2016: 537). Maijala und Tammenga-Helmantel (2016: 540) nennen in diesem Kontext beispielsweise kulturelle Vergleiche, die Lehrende anregen müssen oder zusätzliches Input für die kommunikativen Übungen.

Nicht zuletzt als Folge dieses Defizits entstehen regionale Lehrwerke, die kontrastiv angelegt sind. Rösler (2012: 45) macht darauf aufmerksam, dass bei der Charakterisierung durch das Attribut `kontrastiv` eine große Bandbreite von Realisierungen möglich ist. Sie umfasst

- regionalisierte Lehrwerke, die Adaptionen einsprachiger Lehrwerke darstellen, die u. a. darin bestehen, dass sie z. B. in der Instruktions- und Beschreibungssprache die Sprache der Lernenden aufnehmen sowie

- regionale Lehrwerke, die meist aus der kontrastiven Analyse der Ausgangsund Zielsprache und -kultur das Material aufbauen und im Vorgehen die Sprachlerngewohnheiten und Lerntraditionen der Lernenden in einem bestimmten Land berücksichtigen. (vgl. Rösler 2012: 45)

Dass die Ausgangs- sowie weitere gelernte Sprachen für das Fremdsprachenlernen nicht irrelevant sind, zeigen die Arbeiten u.a. von Butzkamm und Caldwell (2009) sowie von Maijala und Tammenga-Helmantel (2016). Maijala und Tammenga-Helmantel (2016: 558) weisen auf die Vorteile hin, die Nutzung der Ausgangssprache bei der Behandlung von Grammatik mit sich bringt: sie kann die Lernenden dabei unterstützen, »die Sprache(n) zu vergleichen und über die Unterschiede und Gemeinsamkeiten zu reflektieren«. Die Autorinnen weisen auch darauf hin, dass dank dem kontrastiven Einsatz der Ausgangssprache die Palette der Grammatikübungen breiter ist (vgl. Maijala/TammengaHelmantel 2016: 558).

Trotz vieler Vorteile ist das Angebot an regionalen und speziell zielgruppenorientierten im Vergleich zu globalen Produkten eher rar. Wenn man nach diesbezüglichen Gründen sucht, muss der Kostenfaktor erwähnt werden: die Markterwartung einer medialen Vollausstattung von Lehrwerken auf A1 bis B1Niveau führt zu Vorkosten im sechsstelligen Bereich (vgl. Funk 2016: 440). 
Diesen Aufwand können sich hauptsächlich große Verlage leisten und auch nur in solchen Ländern, in denen es entsprechend viele Lernende (lies: Schüler) gibt.

Aber nicht nur ein mangelnder Zielgruppenbezug ist der Grund für die entfachte Diskussion über den Lehrwerkeinsatz. Auch die Ausrichtung neu erscheinender Lehrwerke an normierte Standards, Prüfungen und Kompetenzorientierung bringt Lehrwerke immer wieder in die Kritik.

\section{3 »Zankapfel Lehrbuch« - Diskussion über das Für und Wider der Lehrwerkverwendung im FSU}

Die Diskussion um die Rolle des Lehrwerks im Lernprozess ist so alt wie das Medium selbst. "Zankapfel Lehrbuch« - so der Titel des Beitrags von Gießing (2004) - geriet als Lehr- und Lernmedium besonders im Zusammenhang und infolge der Umsetzung der Forderungen - leider nicht selten missverständlich u. a. nach Autonomie und Authentizität zu Beginn des 21. Jahrhunderts wieder neu in die Kritik (u.a. Freudenstein 2001; Gießing 2003, 2004; Schäfer 2003; Thaler 2004; Vences/Freudenstein 2002). Wie Butzkamm (2005: 93) bemerkt, "[d]as Thema Lehrbücher erinnert uns wieder an die Hauptschwierigkeit des Unterrichts: es allen zugleich recht zu machen«. Vielau (2005: 145) fasst ironisch all das zusammen, was über den Lehrwerkeinsatz kritisch ausgesprochen wird:

Der implizite Lerner ist meistens ungemein fleißig, begreift und weiß sofort alles, was in den Lektionen vorher dran war, ist ein kleiner Linguist, der terminologie-gespickte Regelformulierungen und Arbeitsanweisungen spontan versteht, ist ein umfassend gebildeter Akademiker, der zielkulturelle Spielregeln und Handlungsabläufe aus eigener Erfahrung kennt bzw. intuitiv auffasst, ist experimentierlustig und extrovertiert in Lernspielen, neigt nicht zu Frustration bei viel zu schweren authentischen Hörmaterialien, liebt thematisch anspruchsvolle Texte im Anfängerkurs und kann die Sprache immer gleich anwenden, die er eigentlich erst lernen soll.

Die Kritik des Lehrwerkeinsatzes (für einen Überblick u. a. Funk 2004; Thaler 2011) betrifft vor allem:

- den mangelnden Zielgruppenbezug: die Interessen und Bedürfnisse der Lerner vor Ort sowie ihre Sprachlernerfahrung würden nicht genügend berücksichtigt oder sogar außer Acht gelassen;

- mangelnde Themenvielfalt: die Themenbereiche würden sich in allen Lehrwerken überschneiden ${ }^{3}$, unabhängig davon, für welche Zielsprache das Lehrwerk konzipiert wurde;

3 Der Angleich der Themen in den Lehrwerken dürfte auf den Einfluss des GER zurückzuführen sein. 
- die Progression der Lerninhalte: die inhaltliche und sprachliche Progression wäre an der Grammatik ausgerichtet, Folge wären zu grammatiklastige und zugleich sinnentleerte Texte, wogegen die kommunikativen Aufgaben zu kurz kommen würden;

- Abarbeitungsdruck;

- Kurzlebigkeit: schnell veraltende Texte und Abbildungen;

- Zunahme der Zahl unterschiedlicher Lehrwerke für die gleiche Zielgruppe bei gleichzeitiger Tendenz zur Vereinheitlichung der Inhalte.

Im Jahre 2000 gründete Scott Thornbury ELT Dogme, eine online Diskussionsgruppe, die zum Verzicht auf den Lehrwerkeinsatz im Fremdsprachenunterricht aufrief. In dem im Jahr 2009 erschienenen Buch »Teaching Unplugged: Dogme in English Language Teaching" stellen Meddings und Thornbury zehn Prinzipien für einen Unterricht ohne Lehrwerkeinsatz vor. Auch wenn die Forderung Thornburys (2000: 2): »Teaching - like talk - should centre on the local and relevant concerns of people in the room, not on the remote world of coursebook characters, nor the contrived world of grammatical structures", pädagogisch nachvollziehbar ist, so muss auf die Schwierigkeiten und Gefahren hingewiesen werden, die (gesteuertes) Sprachenlernen ohne jegliche Materialien mit sich bringt. Rösler (2013: 216) sieht in selbstproduziertem Material die Gefahr, »die nötige Komplexität auf thematische Belange zu reduzieren, während professionelle Lehrwerke komplexe Gesamtheiten darstellen, die unterschiedliche sprachsystembezogene Erwägungen (Aussprache, Wortschatz, Grammatik, Pragmatik, landeskundliche Plurizentrik) sowie eine Vielfalt von Übungen und Arbeits- und Sozialformen und eine möglichst lernerrelevante Themenauswahl in eine produktive Balance zu bringen versuchen «. McIver (2009: 419), der eine Besprechung Meddings und Thornburys Buch verfasste, erinnert humoristisch seine erste Erfahrung mit der Welt der Dogme:

Many moons ago, a naive young teacher trainer was awaiting dinner in a rather imposing north Italian hotel restaurant. The proprietor approached:

Prop Buona sera. What would you like?

NYTT Could I see the menu please?

Prop We don't have a menu. We have a chef. He's got it, he cooks it. Now, what would you like?

Disconcerting or what? But I soon got the hang of it ... And it was a wondrous experience! It was also, in retrospect, perhaps my first unwitting entrée into the world of Dogme.

McIver (2009: 421) trifft es auf den Punkt, wenn er konstatiert: »If you are going to get rid of the menu, make sure that you have got a damn fine chef«. Auch Thaler (2011: 27) sieht die >Türklinken-Didaktikı (oder >Auto-Didaktikı) als 
verantwortungslos, eine permanente `DIY^-Erstellung (Do it yourself!) von Arbeitsmaterialien zu zeitaufwändig, unsystematisch - und unprofessionell. Diese Meinung scheinen auch die Schüler zu vertreten. Wie Sambanis (2009: 11) aufgrund ihrer Untersuchung feststellen konnte, ist die Akzeptanz von neueren Lehrwerken unter Sekundarstufenschülern groß. $95 \%$ bis $100 \%$ der von ihr befragten Sekundarschüler gaben an, mit den Lehrwerken (für Englisch und Französisch) zufrieden oder sogar sehr zufrieden zu sein. Auch die workbooks wurden insgesamt positiv von den Schülern bewertet, sie seien "super zum Üben« und »besser als kopierte Arbeitsblätter« (Sambanis 2009: 11).

In der fachdidaktischen Diskussion werden zahlreiche Argumente für einen lehrwerkgestützen Unterricht eingebracht. Besonders im institutionalisierten Kontext, bei dem bestimmte vergleichbare Kompetenzstandards erwartet werden, kann das Lehrwerk »eine Grundlage für die Vergleichbarkeit von Prüfungsergebnissen « bieten, es macht die Unterrichtsinhalte überschaubar, kann die Progression der Lernenden organisieren und überprüfen, den Lernenden als Strukturierungshilfe dienen und selbständiges Lernen auch (ohne Lehrer) außerhalb des Unterrichts ermöglichen (vgl. Maijala 2007: 543f.). Auch Börner et al. (2011: 42) sehen als Vorteil in den Lehrwerken die Struktur. Diese geben individuellen Lernprozessen eine Richtung (Progressionen), indem Lernabläufe durch Units, Themes und spirales Fortschreiten rhythmisiert werden, Lernprozesse durch strukturiertes Lernen optimiert werden und die Beraterrolle des Lehrers optimiert wird (vgl. Börner et al. 2011: 42).

Zweifelsohne besteht heute sowohl unter Praktikern als auch Forschern Konsens darüber, dass die »Heterogenität bei Lehrkräften und Schülern zu groß [ist], das Bedingungskonglomerat aus fachdidaktischen, ökonomischen und administrativen Bedürfnissen zu komplex" (Thaler 2011: 27), als dass ein universelles Lehrwerk entstehen könnte. Die "Gemachtheit» (vgl. Nieweler 2017a: 207) eines Lehrwerkes wird sich zwar nicht leugnen lassen, dennoch bietet ein mündiger Umgang mit dem Lehrwerk viele Vorteile: Nieweler (2010: 14) bezeichnet das Lehrwerk als `katalysierend ‘ bei der Umsetzung neuer didaktischer Erkenntnisse. Funk (2004: 42) sieht in Lehrwerken, »besonders dann, wenn Theorie und Praxis zusammenarbeiten, ein gestuftes, geordnetes, theoretisch fundiertes Lernprogramm [...], eine Leitlinie, die Theorie- und Praxiserfahrung enthält und verfügbar macht, nicht aber ein alles umfassendes Angebot für den Unterricht«. Die vieldiskutierten Verwendungsweisen von Lehrwerken - vom Steinbruch bis zur Unterrichtsdeterminierung - sind nach Rösler (2013:217) in unterschiedlichen Konstellationen unterschiedlich sinnvoll. Er sieht eine Mittelposition als sinnvoll an. Auch Koenig (2010: 180) fordert Lehrkräfte dazu auf, ein Lehrwerk als ein Angebot zu sehen, das Lehrkräfte im Sinne einer Lehrwerkbewusstheit zusammen mit ihren Lernern entdecken und erarbeiten sollten, um das in ihm verborgene Potenzial zu entdecken und nutzen zu können. 
Dazu bedarf es aber eines souveränen Umgangs mit dem Lehrwerk und bestimmter Kompetenzen seitens der Lehrkraft. Es darf nicht vergessen werden, »Unterricht als Lehrer-Lerner-Lehrwerk-Interaktion ist ein komplexes Geflecht interdependenter und kaum separierbarer Faktoren, die in ihrer Summe die Prozess- und Ergebnisqualität von Unterricht ausmachen « (Funk 2010: 366). In ähnlichem Ton spricht sich Waldemar Pfeiffer aus. Er weist darauf hin, dass die fremdsprachigen Lehr- und Lernprozesse komplexer und komplizierter Natur sind und dass sie von vielfältigen Relationen zwischen den am Unterrichtsprozess beteiligten didaktischen Größen abhängen: Lehrer - Schüler - Sprache Lehrwerk - Unterrichtsmethode - Unterrichtsumgebung (vgl. Pfeiffer 2012: 20). »Es sind die Qualitäten und Kompetenzen der Lehrer, die in diesem Bedingungskonglomerat am Erfolg im großen Maße mitentscheidend sind «. Auch Thaler spricht dem Lehrer große Verantwortung zu: »Wie die darin steckenden Potentiale genutzt werden, hängt allerdings nicht vom Lehrbuch ab, sondern von der Lehrkraft, die das Lehrbuch ge- oder missbraucht« (Thaler 2011: 19). Da die Unterrichtspraxis stark durch das jeweils eingesetzte Lehrwerk mitbestimmt wird (vgl. u. a. Luke et al. 1989; Tomlinson 2012; Guerrettaz/Johnston 2013), und ein Lehrwerk per se nicht gut oder schlecht ist, müssen den (zukünftigen) Lehrkräften Orientierungshilfen an die Hand gegeben werden, „damit ein Lehrwerk nicht nur nach Daumenprobe eingeführt wird «(Thaler 2011:25). Eine prominente Rolle bei der Erfüllung dieser Forderung kommt der Lehrwerkanalyse und Lehrwerkkritik zu.

\subsection{Lehrwerkanalyse und Lehrwerkkritik}

Das Lehrmaterialangebot, insbesondere für Deutsch-als-Fremdsprache, ist als groß, wahrscheinlich sogar unüberschaubar groß zu bezeichnen. Trotz oder gerade wegen dieses Umfangs und der Vielfalt stehen Lehrer oft vor der Schwierigkeit, ein geeignetes Lehrwerk für ihren Kontext zu finden. Nach Funk (2016: 437) erwarten die Lehrkräfte von den publizierten Lehrmaterialien eine Unterstützung bei der Untergliederung der Lerninhalte, motivierende Gestaltung und transparente Strukturierung des Lehr- bzw. des Lernprozesses durch die Bereitstellung von Unterrichtsinhalten und Übungsanlagen - auf der Grundlage sprachlehr-/-lernwissenschaftlicher Expertise. Die Einschätzung, inwieweit ein Lehrwerk für eine bestimmte Lernergruppe geeignet ist, soll durch Kriterienraster, Lehrwerkanalysen und -kritiken erleichtert werden.

Die systematische Auseinandersetzung mit Lehrwerken ist in der Geschichte der wissenschaftlichen Forschung im Bereich Deutsch als Fremdsprache relativ jung. Bis in die 1960er Jahre erübrigte sich jede wissenschaftliche Diskussion über Lehrwerke, denn in den führenden Fremdsprachenfächern gab es jeweils 
ein Lehrwerk, das eine Art Monopolstellung einnahm. Mit der beginnenden Pluralität der Lehrmethoden in den 1960er Jahren, im Übergang vom audiolingual/audiovisuellen zum kommunikativen Fremdsprachenunterricht (vgl. Funk 2010: 364) sowie mit den gesellschaftlich-politischen und institutionellen Veränderungen in der Bildungs- und Schulpolitik in den späten 1950er und 1960er Jahren entstanden neue Lehrwerke mit unterschiedlicher didaktischmethodischer Konzeption. In den 1970er Jahren wurde ein Arbeitskreis zur Lehrwerkforschung gegründet, 1977 und 1979 wurden erste Kriterienkataloge für die Analyse von Lehrwerken für Deutsch als Fremdsprache - das Mannheimer Gutachten (Engel et al. 1979) - konzipiert. Im Jahr 1980 folgte ein auf eine konkrete Zielgruppe abgestimmtes Gutachten »Deutsch für ausländische Arbeiter. Gutachten zu ausgewählten Lehrwerken« (Barkowski et al. 1980). In den Folgejahren wurden weitere Kriterienkataloge entwickelt, wie das Hamburger Gutachten für fachsprachliche Lehr- und Lernmaterialien (Beier/Möhn 1981), der Stockholmer Kriterienkatalog (Krumm et al. 1985), der Brünner Kriterienkatalog (Jenkins et al. 1997) oder ein von Funk (2004) erstelltes Raster mit Qualitätsmerkmalen von Lehrwerken.

Inzwischen gehört die Lehrwerkforschung zu einem festen Bestandteil der Fremdsprachenlehr- und -lernforschung, wenn auch in den letzten Jahrzehnten unterschiedlich starkes Interesse an dem Thema in der fachdidaktischen Debatte zu beobachten war und ist.

Unterschiedliche Erkenntnisinteressen haben dazu geführt, dass in Bezug auf Form und Ziele der Lehrwerkforschung zwischen Lehrwerkanalyse, Lehrwerkkritik und Lehrwerkbegutachtung unterschieden wird. Das Ziel der Lehrwerkanalyse ist die hermeneutische "Erforschung von Inhalten und Konzepten von Lehrmaterialien, in der Regel durch quantitative Erhebungen und Evaluation auf der Basis definierter Einzelkriterien oder umfassender Kriterienkataloge (Funk 2010: 364). Nach Nieweler (2017b: 208) ist die Lehrwerkanalyse eine »in erster Linie systematische, oft vergleichende, auch exemplarisch angelegte Untersuchung eines oder mehrerer Gegenstandsbereiche von Lehrwerken«.

Wenn die Lehrwerkanalyse um Lehrwerkbeurteilung ergänzt wird, »mit den Zielen der Aus- und Weiterbildung von Lehrpersonal in Bezug auf methodische Innovationen sowie der möglichen Empfehlung für die Einführung von Lehrmaterialien für eine konkrete Zielgruppe« (Funk 2010: 364), so spricht man von Lehrwerkkritik. Lehrwerkkritik, die weitgehend gleichbedeutend mit der Lehrwerkevaluation ist, beabsichtigt wissenschaftliche Beurteilung der vorgefundenen Sachverhalte (vgl. Nieweler 2017b). Funk (vgl. 1997: 17) sieht als Aufgabe der Lehrwerkkritik, ein valides Raster von Beurteilungskriterien zu entwickeln, die Beurteilung eines Lehrwerkes nach festgelegten Kriterien vorzunehmen und Empfehlungen zum Einsatz des Lehrwerks unter den vorgegebenen zielgruppenspezifischen Bedingungen zu erarbeiten. Ansätze der Lehr- 
werkanalyse und -kritik folgen nach Funk (2010: 364) den drei folgenden Grundschemata:

1) Abgleich eines bestimmten didaktisch-methodischen oder spracherwerbstheoretischen Ansatzes mit der Konzeption und deren Ausführung in einem bestimmten Lehrwerk oder im Vergleich mehrerer Lehrwerke;

2) (Vergleichende) Analyse von Einzelkomponenten in Lehrwerken;

3) Zielgruppen- und schulformorientierte Eignungsstudien, die von mehr oder weniger genau erhobenen Eignungsmerkmalen einer Zielpopulation ausgehen.

Die 1990 von Kast erwähnten Beschränkungen und Aussagen der Lehrwerkkritik haben bis heute nicht an ihrer Aktualität verloren:

- Jedes Beurteilungsraster ist subjektiv;

- Jede Beurteilung aufgrund eines solchen Rasters bleibt subjektiv;

- Eine Gewichtung von einzelnen Kriterien ist von vielen Faktoren abhängig;

- Jede Lehrwerkbeurteilung ist von den Realitäten des DaF-Unterrichts in einem Land, einem Institut, einem Kollegium abhängig. (vgl. Neuner 1994: 22)

Auch wenn Lehrmaterialanalyse seit gut 40 Jahren als eines der zentralen Arbeitsgebiete in der fremdsprachendidaktisch-orientierten Forschung gesehen werden kann, ist damals wie heute ein Defizit an unterrichtsbezogener Forschung zu Lehrwerken zu konstatieren. Lehrwerkforschung geht überwiegend werkanalytisch vor: Lehrwerke werden als Texte oder multimediale Angebote analysiert. Rezeptionsanalytische Zugänge, bei denen es darum geht, wie Lehrende und Lernende mit den Lehrwerken umgehen, sind eher selten (vgl. u.a. Rösler/Schart 2016: 490f.; Garton/Graves 2014: 654; Tomlinson 2013: 44).

In den letzten Jahren entstanden viele weitere Kriterienkataloge und Analysen konkreter Lehrwerke. Es wurden u. a. landeskundliche und interkulturelle Aspekte diskutiert als auch lexikalische oder grammatische Inhalte analysiert ${ }^{4}$, auch der Zielsprachengebrauch sowie die Frage nach der Authentizität der Sprache in den Lehrwerken wurde problematisiert (Hoch et al. 2016).

Die dritte Dimension der Lehrwerkforschung stellt die Lehrwerkbegutachtung dar. Als Ziel der Lehrwerkbegutachtung wird Einführungs- bzw. Freigabeempfehlung bzw. deren Verweigerung in Bezug auf eine konkrete Zielgruppe genannt (vgl. Funk 2010: 364). Das Zulassungsverfahren (wenn es überhaupt

4 U.a. Ciepielewska-Kaczmarek (2016) zu Landeskunde, Maijala/Tammenga-Helmantel (2017) zu Grammatikübungen; Tammenga-Helmantel/Maijala (2018a, b) zu der Position von Grammatik bzw. Grammatikprogression; Maijala/Tammenga-Helmantel/Donker (2016) und Tammenga-Helmantel/Ciepielewska-Kaczmarek/Jentges (2017) zu Kultur/Landeskunde. 
eins gibt) für den Einsatz eines Lehrwerks an Schulen läuft in nahezu jedem Land anders. Beispielsweise in Deutschland, einem bildungsföderalen Staat, lassen sich in Bezug auf die Lehrwerkzulassung gravierende Unterschiede feststellen. In jedem der 16 Bundesländer entscheiden die jeweiligen Kultusministerien mit Hilfe unabhängiger Gutachter über die Einführung eines neuen Lehrwerkes. In den Niederlanden gibt es kein entsprechendes Verfahren, während die Lehrwerke in Polen einem landesweiten staatlichen Zulassungsverfahren unterliegen.

Unabhängig davon, wer über die Einführung eines Lehrwerkes entscheidet,

Classroom materials are an integral part of second language classrooms. Teachers everywhere rely, often heavily, on textbooks, workbooks, reference materials, and other kinds of materials as sources of linguistic input, explanations of language, and opportunities for students to practice new or previously learned language. (Guerrettaz/ Johnston 2013: 779)

Kurtz (2011: 6) hebt hervor, „Optimale Lehrwerkverwendung bedeutet nicht maximale Lehrwerkbindung«. Das Lehrbuch gilt nicht als ein »seitenumblätterndes Medium ", sondern als "Sammlung von Unterrichtsbausteinen, der man folgen kann, die man aber auch in Abstimmung auf die Lernergruppe durch geeignete Materialien ergänzen bzw. ersetzen kann« (Nieweler 2017a: 207). Diese Kompetenz muss aber gelernt werden. Lehrwerkanalysen und -kritik sollten aus diesem Grund im Lehrplan der Lehrerausbildung fest verankert sein. Die angehenden Lehrkräfte sollten für einen kritisch-reflektierten Umgang mit Lehr-/Lernmaterialien sensibilisiert und befähigt werden, die vorliegenden Materialien entsprechend der Bedürfnisse der Lerner vor Ort möglichst gut anzupassen bzw. einzusetzen.

Eine besondere Rolle in der Lehrwerkanalyse kommt dem landeskundlichen Aspekt zu, denn beim Sprachenlernen kommt man an der Kultur nicht vorbei, wie Krumm (1998: 524) betont, "Sprachenlernen ist immer Kulturlernen«. Die $\mathrm{zu}$ vermittelnden landeskundlichen und interkulturellen Inhalte, die zum Aufbau interkultureller Kompetenzen führen sollen, werden in den Lehrwerken verschieden umgesetzt. Lütge (2016: 458) sieht eine wichtige Aufgabe der Lehrkräfte darin, bestehende Lehrwerke kritisch zu prüfen, selbständig Lernmaterialien zu kombinieren und passende Aufgabenstellungen zu entwickeln, die eine Bewusstmachung interkultureller Thematiken unterstützen.

Nach einer Übersicht der Geschichte der Landes- und Kulturkundeforschung und deren Entwicklung im Bereich der Fremdsprachendidaktik wird in Kapitel 4 die konkrete Umsetzung von landeskundlichen Inhalten in jeweils drei Lehrwerken für Deutsch als Fremdsprache in den Niederlanden und in Polen analysiert. Für eine bessere Verständlichkeit und einen besseren Vergleich wird 
im Folgenden die verwendete Terminologie in Bezug auf die Struktur der Lehrwerke geklärt.

Unter Medienverbund verstehen wir die Gesamtheit aller Komponenten, die zu einer Lehrwerkreihe gehören, darunter Kurs- und Arbeitsbuch, CDs, Lehrerhandbuch als auch weitere Zusatzangebote, die entweder als Print oder digital vorliegen, wie z. B. optionale Grammatik- und Testhefte, DVDs, Lernposter usw. $=$ Lehrwerk sensu largo.

Das Lehrwerk sensu stricto (auch Lehrwerkreihe) bilden diejenigen Bestandteile, die im Unterricht obligatorisch eingesetzt werden. Dazu gehören Kurs- und Arbeitsbuch, CDs und Lehrerhandbuch.

Das Kursbuch (im Deutschen auch Schülerbuch, Lehrbuch, im Polnischen podręcznik) bietet Lese- und Hörtexte, Wortschatz- und Grammatikübersichten und Aufgaben. Ein Kursbuch besteht aus mehreren (meist thematischen) Einheiten (auch Kapitel). Die Kapitel sind wiederum in kleinere Lektionen unterteilt.

Das Arbeitsbuch enthält meist vertiefende Übungen für die eigenständige Arbeit, z. B. zu Hause.

Für die Niederlande sieht die Zusammenstellung eines Lehrwerkes etwas anders aus. Die Lernenden arbeiten sowohl zu Hause als auch in der Schule mit einem Textbuch, das Texte, Grammatik und Wortschatz bietet, und einem Arbeitsbuch, das die Aufgaben zum Textbuch enthält. Manchmal bieten Verlage ein integriertes Text-Arbeitsbuch an. Optionale oder vertiefende Aufgaben werden nicht, wie in Polen und Deutschland, als Arbeitsbuch angeboten, sondern stehen den Lernenden als digitales Angebot zur Verfügung. 


\section{Landeskundliche und kulturelle Inhalte im Fremdsprachenunterricht}

Publikationen zur und über die Vermittlung landeskundlicher ${ }^{5}$ Inhalte im Deutsch-als-Fremdsprache-Unterricht existieren heutzutage (zumindest im deutschsprachigen Raum) zahlreich. Auch in Lehrwerken stellen landeskundliche und kulturelle Aspekte einen festen Teil der Inhalte dar. Die Relevanz solcher kulturellen Inhalte dürfte weder von Lehrkräften noch Fremdsprachendidaktikern in Frage gestellt werden, geht das Lernen einer Sprache doch immer - bewusst oder unbewusst - auch einher mit dem Lernen über oder von kulturellen Inhalten, die die jeweiligen zielsprachigen Lebenswelten prägen und letztlich bleibt Sprache »schon in ihrer Bedingung für die Möglichkeit von Erfahrung und Denken, immer Teil, ja Ausdruck der Kultur « (Bleyhl 1994: 9). Und auch im GER wird der kulturelle Kontext abgedeckt, in den eine Sprache eingebettet ist, und spielt Kultur, insbesondere im so genannten interkulturellen Kontext eine wichtige Rolle:

Als sozial Handelnder geht jeder Mensch Beziehungen mit einem sich ständig erweiternden Geflecht überlappender sozialer Gruppen ein, was insgesamt seine >Identität definiert. In einem interkulturellen Ansatz ist es ein zentrales Ziel fremdsprachlicher Bildung, eine günstige Entwicklung der gesamten Persönlichkeit des Lernenden und seines Identitätsgefühls als Reaktion auf die bereichernde Erfahrung des Andersseins anderer Sprachen und Kulturen zu fördern. (GER, Kapitel 1.1)

5 Trotz aller und zweifelsohne auch berechtigter Kritik an dem Begriff "Landeskunde« verwenden wir diesen, da er im Rahmen der unterrichtspraktischen Diskussion gängig ist. »Der Begriff Landeskunde ist $[\ldots]$ nicht ganz unproblematisch: erstens, weil der Begriff "Land « in der Regel mit einem Festhalten an nationalstaatlichen Grenzen einhergeht und daher am ehesten noch mit dem [...] faktenbezogenen Ansatz vereinbar wäre; zweitens, weil je nach argumentativen Standpunkt die Zusammenhänge zwischen Landeskunde und weiteren im fremdsprachenwissenschaftlichen Diskurs verwendeten Begriffen unterschiedlich bestimmt werden kann." (Hoch 2016: 4). Gemeint ist hiermit, dass unterschiedliche Auffassungen vorliegen, ob Landeskunde beispielsweise als Vorläufer des interkulturellen Lernens zu sehen ist, als parallel neben diesem existierenden Konzept oder als integrativer Bestandteil von interkulturellem Lernen (vgl. Koreik/Pietzuch 2010: 1447). 
Trotz dieser unumstrittenen Bedeutung, die Kultur für das Sprachenlernen zugeschrieben werden kann, ist festzuhalten, dass die Vermittlung kultureller Aspekte der Zielsprachengebiete keineswegs länderübergreifend fest in Lehrplänen oder Rahmencurricula verankert ist. So findet sich beispielsweise in den curricularen Vorgaben für den DaF-Unterricht in Polen lediglich ein allgemeiner Verweis auf die Vermittlung von Elementen der zielsprachigen und der eigenen Kultur(en), im Sinne eines interkulturellen Kontextes und der europäischen Integration $^{6}$; in den niederländischen zentral geregelten Abschlussqualifikationen fehlt ein solcher Hinweis auf Landes- und Kulturkunde gänzlich (vgl. Tammenga-Helmantel et al. 2017: 32). Eine grundlegende Frage, die sich nicht nur den Verfassern offizieller bildungspolitischer Vorgaben, sondern ebenso Lehrwerksautoren sowie Fremdsprachenlehrkräften stellt, und die durchaus auch in der Fremdsprachendidaktik kontrovers diskutiert wird, ist die, was überhaupt unter Kultur und Landeskunde im Fremdsprachenunterricht zu verstehen ist und welche Konzepte sowie Inhalte hinter ausgewählten landeskundlich-kulturellen Aspekten im Fremdsprachenunterricht stehen sowie welche Position Landeskunde in einem Lehrwerk einnimmt.

\subsection{Landes- und Kulturkunde: Inhalte und Ansätze}

Es gibt sowohl in der Unterrichtspraxis von Lehrpersonen als auch im fachwissenschaftlichen Diskurs der Fremdsprachendidaktik sehr unterschiedliche Ansichten darüber, was Landes- und Kulturkunde im Fremdsprachenunterricht ist, sein kann oder sein könnte; bekannt dürften vor allem diejenigen Aussagen sein, die Landeskunde auf die eine oder andere Weise als Problem bezeichnen. Landes- und kulturkundliche Inhalte im Fremdsprachenunterricht sind ein kaum einzugrenzendes Gebiet, das sich zudem noch ständig in Veränderung befindet. So ist Mog sicher auch heute noch zuzustimmen, wenn er konstatiert:

Die Landeskunde steht vor einer unlösbaren Aufgabe: Sie soll Fremde mit der Geschichte, den politischen Strukturen und Institutionen, den sozialen und kulturellen Verhältnissen, mit Philosophie, Literatur und Kunst eines Landes vertraut machen, sie soll selbstverständlich auch Einblick in die Mentalität, die Lebensweise und den Alltag seiner Bewohner geben. Selbst wenn es so enzyklopädisch Gebildete unter den Sprachund Landeskundelehrenden gäbe, ihre Aufgaben sind damit noch keineswegs er-

6 Vgl. Rahmencurriculum für moderne Sprachen: https://podstawaprogramowa.pl/Szkolapodstawowa-IV-VIII/Jezyk-obcy-nowozytny. Hier heißt es: Der Schüler verfügt über: 1) Grundkenntnisse über Länder, Gesellschaften und Kulturen von Gemeinschaften, die eine bestimmte Fremdsprache verwenden, und über das Heimatland, einschließlich des lokalen, europäischen und globalen Kontexts, 2) das Bewusstsein für den Zusammenhang zwischen eigener und fremder Kultur und interkulturelle Sensibilität. 
schöpft. Sie haben ja nicht nur Wissen zu vermitteln, sie sollen darüber hinaus zugleich Vorurteile abbauen, Stereotype auflösen, Völkerverständigung fördern; sie sollen über das Fremdverstehen die Selbsterfahrung der ausländischen Lernenden schärfen, Haltungen und Wahrnehmungen verändern. (Mog 1992: 9)

Das große Spektrum an Anforderungen sowie die beträchtliche Themenvielfalt für den landes- und kulturkundlichen Fremdspracheunterricht sind kaum und noch weniger zur gleichen Zeit in der Unterrichtspraxis einzulösen. Auch die traditionell sehr unterschiedlichen Auffassungen darüber, was Landeskunde ist, lassen sich in großen Teilen im obigen Zitat von Mog wiederfinden, nämlich Landeskunde im Sinne von kognitivem Faktenwissen, meist bezogen auf die so genannte hohe Kultur (großes »K«) und Landeskunde im Sinne von praktischem Alltagswissen und kommunikativen Strategien bezogen auf Alltagskultur (kleines »k«). Darüber hinaus fordert der seit Ende der 1980er Jahre für den Fremdsprachenunterricht populär gewordene interkulturelle Ansatz (vgl. Bausch et al. 1994), Fremdverstehen und Selbstreflexion $\mathrm{zu}$ initiieren. Grob vereinfacht können mit Pauldrach (1992) stets noch drei klassische Ansätze der Landeskunde-Didaktik unterschieden werden. Diese drei Konzepte, die jeweils Tatsachenvermittlung (faktisch-kognitiver Ansatz), kommunikative Kompetenz im Alltag (kommunikativer Ansatz) und kommunikative Kompetenz in interkulturellen Interaktionssituationen (interkultureller Ansatz) in den Mittelpunkt stellen, lassen sich am deutlichsten in älteren Lehrbüchern wiederfinden und unterscheiden (vgl. Cerri/Jentges 2015b: 99-106; Schweiger et al. 2015: 3):

- Faktisch-kognitiver Ansatz: Im Mittelpunkt steht die Vermittlung beispielsweise politischer, wirtschaftlicher, geographischer oder kultureller Fakten, die scheinbar ein objektives Bild der zielsprachigen Kultur zeigen. Es geht um die Präsentation der »hohen Kultur« oder touristischer Sehenswürdigkeiten, zu denken wäre an eine Lebensbeschreibung Goethes, das politische System eines deutschsprachigen Landes, eine Übersicht von Größe, Einwohnerzahl und Landeshauptstädten der 16 deutschen Bundesländer oder eine mit Fakten unterbaute Präsentation der Sehenswürdigkeiten einer Stadt im deutschsprachigen Raum. Oft werden solche Lehrbuchtexte dann kontextlos in den Dienst grammatischer Übungen gestellt, z. B. um Adjektivendungen oder Verben im Präteritum zu behandeln, ohne überhaupt auf die dargebotenen Inhalte einzugehen. Die Vorstellung solcher Inhalte ermöglicht es, dass Lernende Fakten über die Zielsprachenkultur(en) erhalten, bleibt es jedoch bei diesem reinen Faktenwissen, erhalten sie aber keinen wirklichen Einblick in das Leben vor Ort oder Hinweise zur Bewältigung des Alltags dort.

- Kommunikativer Ansatz: Im Mittelpunkt stehen kommunikative Handlungsbeispiele aus dem Alltagsleben, das Spektrum ist breit, vom Tomatenkauf auf dem Markt, über die sprachliche Realisierung von Geburtstags- 
glückwünschen in der Zielsprache bis hin zu einer Panne auf der Autobahn. Präsentiert wird, was einem vor Ort in der zielsprachigen Umgebung als Sprachhandlung begegnen könnte. Praktische Handlungssituationen aus dem Alltag, im Sinne vom kleinen »k«, werden mit den dazugehörigen sprachlichen Mitteln vermittelt. Fokus auf die Beherrschung von Redemitteln und Orientierungsfähigkeit im Alltag zeichnen diesen Ansatz aus, in dem präsentierte Inhalte vor allem als Mittel zum Zweck der Umsetzung dieser kommunikativen Ziele zu sehen sind.

- Interkultureller Ansatz: Persönliche Wahrnehmung, Sensibilisierung für vermeintlich Eigenes und Fremdes sowie Reflexion hierüber werden in den Mittelpunkt gestellt. Die Reflexion soll über rein kontrastives Gegenüberstellen der Ausgangs- und Zielkulturen hinausgehen und somit zu einem besseren Verständnis der zielsprachigen Kulturen, ebenso zu einer Reflexion der eigenen Kultur anregen. Grundlegender Gedanke zur Vermittlung interkultureller Kompetenz im Fremdsprachenunterricht ist hierbei, dass wir auf Basis unserer Werte, unseres Vorwissens und unserer Erfahrungen ständig interpretieren. Wahrnehmung wird somit als »aktive, perspektivische Konstruktion der jeweiligen subjektiven Wirklichkeit des Individuums" verstanden und ist hierdurch immer selektiv, persönlich und gruppenspezifisch, eine aktive Interpretation und von den eigenen Erfahrungen geprägte (evtl. kulturspezifische) Wertung (vgl. Biechele/Meinert 2001: 10). Aus fremdsprachendidaktisch orientierter Perspektive wird interkulturelle Kompetenz daher in der Regel als aus mindestens drei Teilbereichen bestehende Handlungskompetenz beschrieben:

- kognitive Dimension (knowledge): Kulturvergleich und kognitives Wissen,

- affektive Dimension (attitudes): Wahrnehmungsschulung und Bedeutungserschließung,

- pragmatische Dimension (skills): Kommunikationsfähigkeit in interkulturellen Situationen.

Wesentlich ist, dass diese drei Teilkompetenzen ineinander übergreifen und nur im Zusammenspiel zu Handlungsstrategien entwickelt werden können, um eventuell fehlende gemeinsame Standards oder Scripts auszugleichen oder neue auszuhandeln.

Auf sprachlicher Ebene wäre hier beispielsweise an denotative wie auch konnotative - oft kulturgeprägte - Bedeutung von Wörtern oder Floskeln zu denken, inhaltlich daran, dass bestimmte gesellschaftliche oder zwischenmenschliche Ereignisse unterschiedlichen Normen und Erwartungen unterliegen: Wem gratuliert man zum Geburtstag? Was wünscht man sich? Was schenkt man? Wie und mit wem feiert man? Letztlich liegt dem interkulturellen Ansatz eine Integration von sowohl faktischem als auch kommunikativem Wissen zu 
Grunde (vgl. u. a. Zeuner 2010: 1472; Rösler 2012: 204; Schweiger et al. 2015: 3), welches für kulturübergreifende Handlungskompetenz ebenso wichtig ist, wie eine durch Sensibilisierung zu erreichende Fähigkeit zur Empathie vermeintlich Eigen- und Fremdkulturellem gegenüber (siehe hierzu auch Absatz 3.5 und 3.5.1).

Kurz zusammengefasst lässt sich festhalten, dass der faktisch-kognitive Ansatz auf die Vermittlung von Faktenwissen abzielte, die kommunikative Landeskunde sprachliches und situationsangemessenes Zurechtfinden im zielsprachigen Alltag in den Mittelpunkt rückte und interkulturell geprägte Ansätze auf Orientierung zwischen und Vergleich von Eigenem und Fremden fokussierten. Die dargestellten klassischen Ansätze der Landeskunde finden sich in aktuellen DaF-Lehrbüchern mittlerweile nicht mehr in reiner Form (vgl. Cerri/ Jentges 2015b: 106), sondern in einer produktiven Verbindung zueinander, häufig mit einer stärkeren Tendenz zur kommunikativen Landeskunde. Darüber hinaus entwickelt sich immer stärker der Trend, dass landes- und kulturkundliche Inhalte implizit im gesamten Lehrwerk umgesetzt werden und nicht nur in gesonderten Teilen auftreten, die explizit mit der Überschrift "Landeskunde» versehen sind (vgl. Rösler 2012: 198).

\subsection{ABCD und DACH: Integration der Vielfalt}

Zumindest globale, aktuelle Lehrbücher stützen sich außerdem - was die konzeptionelle Gestaltung angeht - oft auf die ABCD-Thesen (vgl. u.a. ABCDThesen 1990; Krumm 1998; Clalüna et al. 2007; Pucharski 2009; Demmig et al. 2013; IDV-Magazin 2017, hier insb. Krumm 2017), die vor fast dreißig Jahren verbindliche methodisch-didaktische Prinzipien für Landeskunde im DaFUnterricht festlegen wollten, und dem hieraus hervorgegangenen $\mathrm{DACH}(\mathrm{L})$ Konzept (vgl. Krumm 2017: 8). Die 22 ABCD-Thesen wurden von einer Arbeitsgruppe bestehend aus Vertretern der Deutschlehrerverbände aus der BRD, der DDR, aus Österreich und der Schweiz erarbeitet und 1990 publiziert. Landeskunde wird hierbei als »ein Prinzip« im Fremdsprachenunterricht gesehen,

das sich durch die Kombination von Sprachvermittlung und kultureller Information konkretisiert und durch besondere Aktivitäten über den Deutschunterricht hinaus wirken soll, z. B. durch Austausch und Begegnung. Insofern ist Landeskunde kein eigenes Fach.

Landeskunde ist nicht auf Staaten- und Institutionenkunde zu reduzieren, sondern bezieht sich exemplarisch und kontrastiv auf den deutschsprachigen Raum mit seinen nicht nur nationalen, sondern auch regionalen und grenzübergreifenden Phänomenen. Ein solches Verständnis von Landeskunde widerspiegelt das Konzept des sich herausbildenden »Europa der Regionen«. (ABCD-Thesen 1990: 15) 
Landeskunde wurde demnach als in der Sprachvermittlung integriertes Prinzip proklamiert, das exemplarische, realistische Einblicke in die deutschsprachigen Länder gibt und durch Interkulturalität sowie Kontrastivität, durch authentische Materialien, projektähnliche Aufgaben und lernerorientierte Unterrichtsgestaltung geprägt ist. Einen wichtigen Aspekt bei den Postulaten der ABCD-Arbeitsgruppe bildet auch die Integration der Vielfalt und regionaler Varietäten auf sprachlicher und kultureller Ebene, nämlich im Sinne einer Integration der verschiedenen deutschsprachigen Länder, in den DaF-Unterricht. So lautet beispielsweise These 5:

Im Deutschunterricht und daher auch in Lehrwerken und Zusatzmaterialien müssen Informationen über den ganzen deutschsprachigen Raum berücksichtigt werden. Dabei sind Vielfalt der Quellen und Transparenz der jeweiligen Standpunkte und Sichtweisen wichtige Kriterien. (Ebd.: 16)

Die zu berücksichtigende Vielfalt an Informationen wird in weiteren Thesen näher spezifiziert und in These 12 auf Sprache sowie in These 15 auf kulturelle Aspekte im weiteren Sinne bezogen:

Die Vielfalt von regionalen Varietäten der deutschen Sprache stellt eine wichtige Brücke zwischen Spracherwerb und Landeskunde dar.

Diese Vielfalt darf nicht zugunsten einheitlicher Normen (weder phonologisch noch lexikalisch noch morpho-syntaktisch) aufgegeben, sondern soll für die Lernenden am Beispiel geeigneter Texte und Materialien erfahrbar werden. (Ebd.: 17)

Ein Charakteristikum der deutschsprachigen Kultur(en) ist, daß sie nicht auf einen Mittelpunkt zentriert ist (sind), sondern sich gerade durch die Vielfalt der deutschsprachigen Regionen, durch ihre bei vielen Gemeinsamkeiten auch unterschiedliche historische, politische, kulturelle und sprachliche Entwicklung auszeichnen.

Sich im Unterricht nur auf eine deutschsprachige Region zu beschränken, widerspricht dem Sinn der Landeskundevermittlung. (Ebd.: 17)

In den abschließenden Thesen wird dann ausdrücklich die Berücksichtigung der Vielfalt der deutschsprachigen Regionen bei Materialerstellung (These 19) sowie im Rahmen der Lehreraus- und -fortbildung (These 21) formuliert, explizit wird für beide Bereiche eine Zusammenarbeit bzw. aktive Beteiligung von Fachleuten verschiedener deutschsprachiger Länder eingefordert. Tatsächlich wurden auf die Empfehlungen der ABCD-Arbeitsgruppe insgesamt drei LandeskundeBände für die DACH-Länder samt einem Begleitband entwickelt (vgl. Clalüna/ Langner 1998; Fischer et al. 1998; Koch 1999; Mitzschke 1998), die vor allem faktisches Hintergrundwissen zu Themen wie beispielsweise Geografie und Geschichte, Bevölkerung oder Bildungswesen des jeweils einzelnen Landes präsentieren und durch ihren parallelen Aufbau zu Vergleichen zwischen den Ländern einladen wollen (vgl. Krumm 2017: 8). Auch in den letzten Jahren erschienen verschiedene Zusatzmaterialien, die landeskundliche Materialien zu 
Deutschland, Österreich und der Schweiz anbieten (u. a. Berger/Martini 2005; Faigle 2006, 2007; Pilaski et al. 2011; Seiffert 2009; Specht et al. 2012; Bayerlein 2013; Matecki 2017). Hierbei handelt es sich um Materialien, denen unterschiedliche Landeskunde-Ansätze zu Grunde liegen, beispielsweise dem eher kommunikativ orientierten und Sprach- und Landeskundearbeit verknüpfenden Band Landeskunde aktiv: Praktische Orientierungen für Deutschland, Österreich und die Schweiz (vgl. Abbildung 1) oder dem eher faktisch-kognitiv orientierten und als Lesebuch bezeichneten Band Dreimal Deutsch. In Deutschland - In Österreich - In der Schweiz (vgl. Abbildung 2).

Letztlich handelt es sich bei einer gemeinsamen und alle deutschsprachigen Regionen integrierenden Materialerstellung um ein Postulat, das bis heute gültig ist und inzwischen als so genanntes DACH-Prinzip (vgl. u. a. Clalüna et al. 2007: 38ff.; Studer 2013: 69f.; Shafer/Baumgartner 2017: $67 \mathrm{ff}$.) bezeichnet wird: Ziel ist »die grundsätzliche Anerkennung der Vielfalt des deutschsprachigen Raumes« (IDV 2013). Dass das DACH-Prinzip bis heute in der Diskussion steht, sich 2007 eine (neue) DACHL-AG - bestehend aus Vertreterinnen und Vertretern von Deutschlehrerverbänden, Mittlerorganisationen sowie Experten und Expertinnen - gründete (vgl. Jarząbek 2017: 15), und gleichzeitig teils als nicht mehr zeitgemäß bezeichnet wird (vgl. Altmayer 2013: 15ff.), legt nahe, dass diese Forderung noch nicht ausreichend bzw. angemessen in der Unterrichtspraxis und in Lehrmaterialien umgesetzt wird. Fraglich bleibt, inwiefern ein Ansatz, der vor allem auf Nationalstaaten fokussiert, überhaupt der - nicht nur regionalen - plurilingualen und -kulturellen Vielfalt im deutschsprachigen Raum also auch in den einzelnen DACH-Ländern - gerecht werden kann (vgl. Fornoff/ Koreik, im Druck). Auch die Annahme, DACH-Konzept bzw. die Berücksichtigung von Plurilingualität und -kulturalität hätten sich heutzutage in Lehrplänen und Rahmencurricula für den Fremdsprachenunterricht verankert, ist ein Trugschluss (vgl. Tammenga-Helmantel et al. 2017: 32f.). Zwar zeigt sich, dass DACH bzw. plurizentrische bzw. pluriareale Varianten durchaus Berücksichtigung in Lehrmaterialien finden, dies erfolgt jedoch meist in additiver Form und nicht integrativ, so dass DACH-Inhalte oft kontextlos nebeneinander gestellt und somit in beliebiger und unsystematischer Form präsentiert werden (vgl. u.a. Tammenga-Helmantel et al. 2017: $33 \mathrm{ff}$.). Das DACH-Prinzip scheint bis heute additives Extra, ein möglicher, zur Wahl stehender Zusatz in der Praxis des DaFUnterrichts zu sein. DACH bzw. die Integration der (nicht nur regionalen) plurilingualen und -kulturellen Vielfalt im gesamten Zielsprachengebiet als allgemeiner fachlicher Grundsatz dürfte nur in wenigen Klassenzimmern Realität sein (vgl. u. a. IDV-Magazin 92, hier insb. Shafer/Baumgartner 2017). Anregungen, wie die deutschsprachigen Länder mit einfachen Mitteln in den Unterricht einbezogen werden können, finden sich beispielsweise auch in anderen Zusatzmaterialien, wie dem aus den 1990er Jahren stammenden Materialien- 


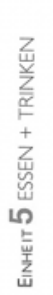

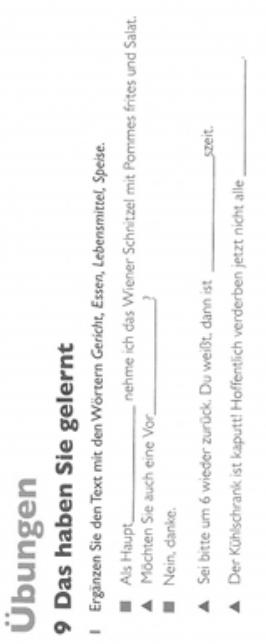

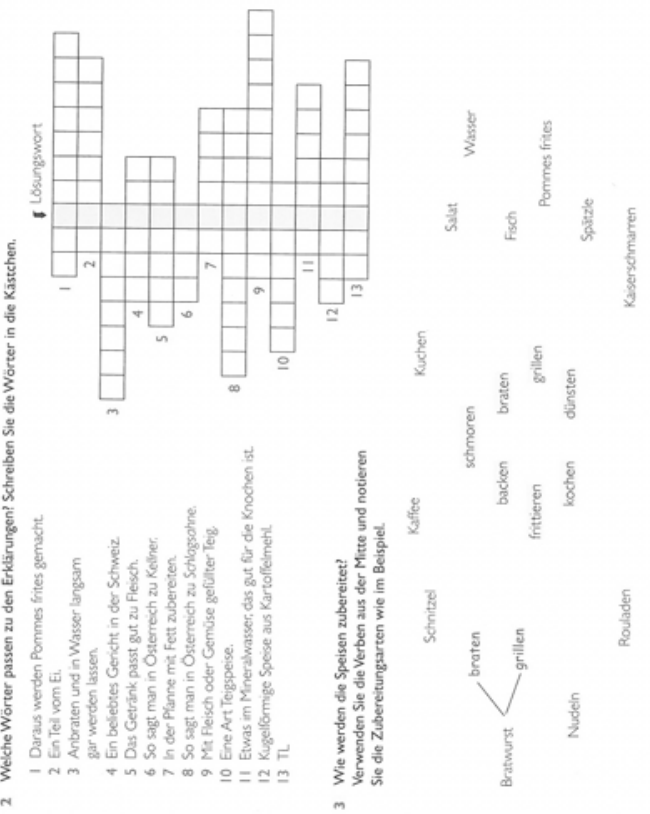
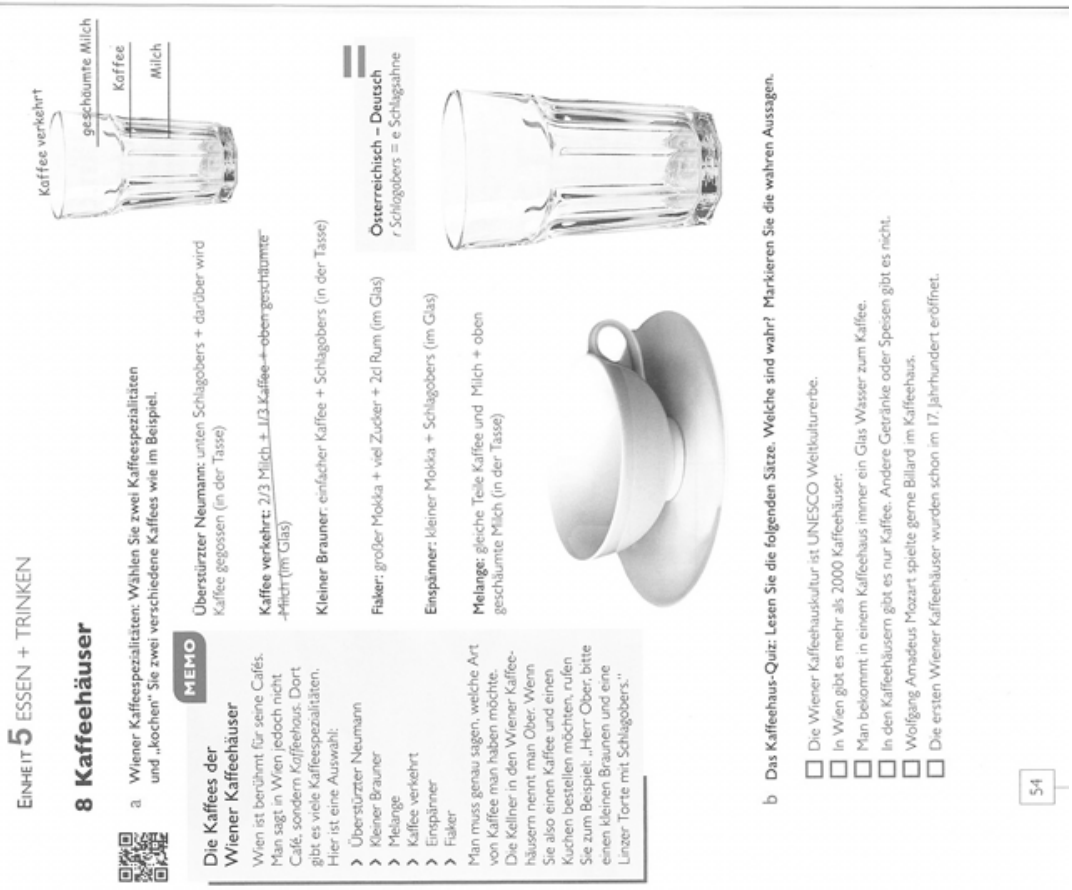

Abbildung 1: Kaffeehäuser (aus: Bayerlein 2013: 54-55) 


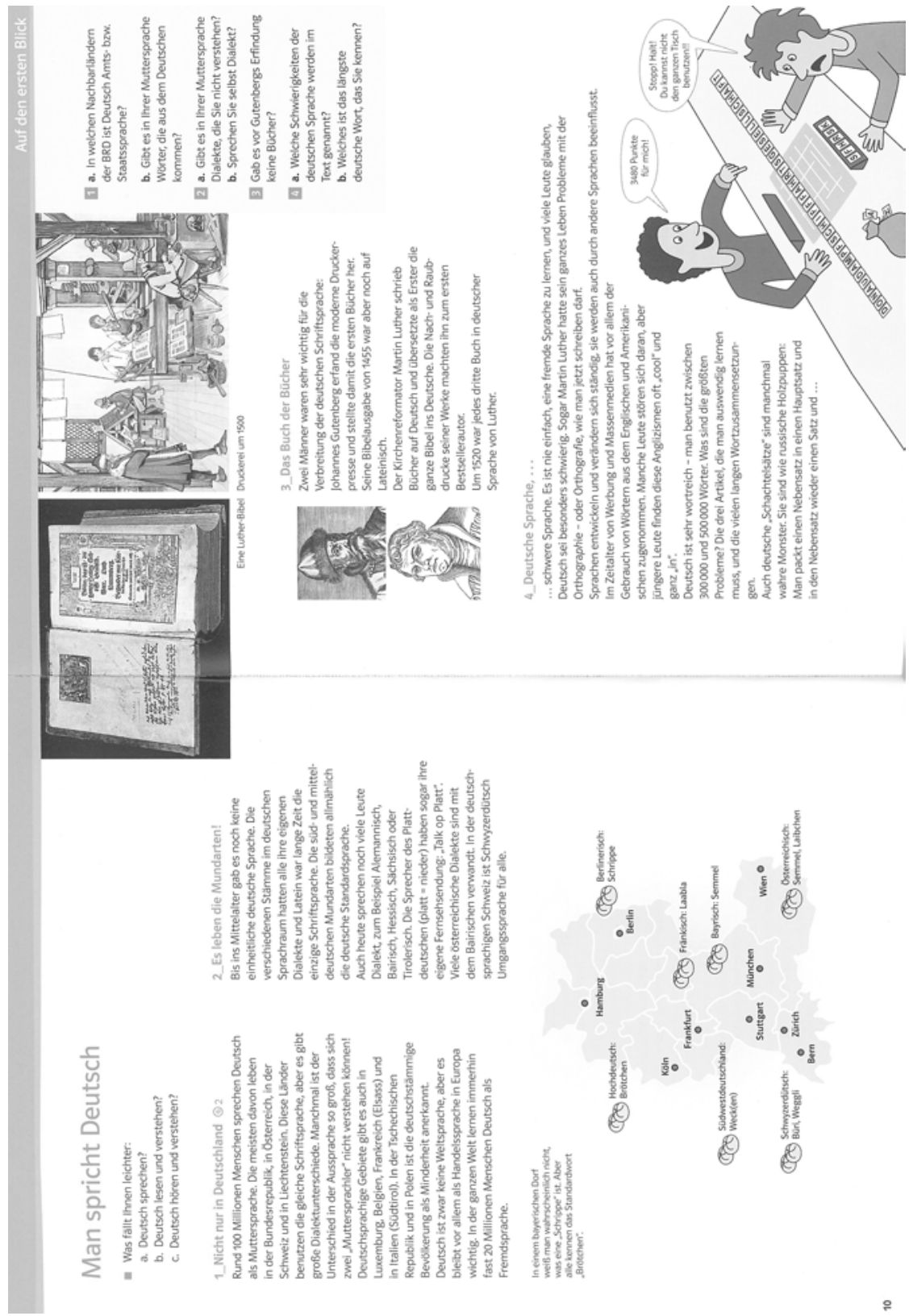

Abbildung 2: Man spricht Deutsch (aus: Matecki 2017: 10-11) 
band Zwischen den Kulturen. Strategien und Aktivitäten für landeskundliches Lehren und Lernen (Hansen/Zuber, vgl. Abbildung 3).

Ob das DACH-Prinzip als allgemein gültiger Grundsatz im DaF-Unterricht umgesetzt werden kann und vor allem, wie dann eine systematische Auswahl von DACH-Inhalten, die nicht beliebig bleibt, erfolgen kann, dürfte - genau wie die Auswahl jeglicher landes- und kulturkundlicher Inhalte (vgl. Rösler 2012: 195ff.) - vor allem von den Rahmenbedingungen des jeweiligen DaF-Unterrichts, also dessen Zielgruppe, Lernort, den Zielsetzungen bzw. Lernzielen und Curricula, den zur Verfügung stehenden Lehrmaterialien, der durchführenden Institution sowie insbesondere der Lernenden und Lehrenden abhängig sein (vgl. Abschnitt 3.3). Welche sprachlichen oder kulturellen Varianten dabei berücksichtigt werden, sollte entsprechend dieser Rahmenbedingungen beschrieben werden, zumindest solange der Unterricht varietätsoffen und pluritätssensibel verläuft und die didaktisch-methodischen sowie inhaltlichen Entscheidungen auf dieser Basis nachvollziehbar sind.

\subsection{Zielgruppen-Orientierung als Prinzip}

Neben der bis heute andauernden Diskussion um die Integration des DACH(L)Konzeptes in den DaF-Unterricht wurde seit Mitte der 1990er Jahre auch über eine fehlende Wissenschaftlichkeit der Landeskunde diskutiert. Frühere Urteile darüber, was Landeskunde sei - ein »Unfach « (Schmidt 1977), ein »unmögliches Fach aus Deutschland " (Gürttler/Steinfeld 1990), eine »unendliche Geschichte» (Pauldrach 1992), das "Monster von Loch Ness der Fremdsprachenphilologie» (Picht 1995) - werden weiterhin als zutreffend angesehen, noch 2007 räumt Altmayer (2007: 8) ein: "Die Landeskunde ist ein Problem«.

Schon diese kurze Auswahl problematisierender Aussagen bezüglich landesund kulturkundlicher Inhalte im Fremdsprachenunterricht sowie mannigfaltige Anforderungen an Inhalte und Zielsetzungen von Landeskunde und Kultur im Unterricht zeigt, dass die Vielfalt von möglichen inhaltlichen Ausrichtungen des Landeskunde-Unterrichts für in der Praxis tätige Lehrpersonen eher Verwirrung-stiftend als Orientierung-bietend wirken kann. So hat Nuffic, die offizielle niederländische Institution für Internationalisierung im Bildungsbereich, in den zwei letzten Jahren im Rahmen des LinQ-Programms ${ }^{7}$ gemeinsam mit einer

7 Programm für Deutsch- und Französisch-Unterricht an weiterführenden Schulen in den Niederlanden, bei dem folgende Basisprinzipien für den schulischen Fremdsprachenunterricht formuliert sind: Zielspracheneinsatz, kommunikativer Unterricht, authentische Sprachsituationen, Integration von Landes- und Kulturkunde und Aktualität, Einsatz von europäischen Sprach(förder)instrumenten (vgl.: https://www.nuffic.nl/onderwerpen/star ten-met-linq/). 


\section{Wie soll dos Kind denn beißen?}

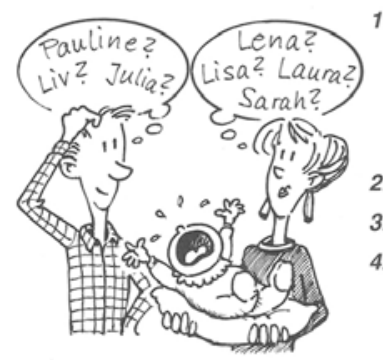

1. Einigen Sie sich in Ihrer Kleingruppe auf drei der unten aufgeführten Aussagen, die Ihrer Meinung nach

a) für Deutschland, b) für die Schweiz, c) für Österreich stimmen, und drei Aussagen, die nicht für diese Länder stimmen. Tragen Sie die Ergebnisse in das Raster ein.

2. Berichten Sie Ihre Ergebnisse im Plenum.

3. Vergleichen Sie Ihre Ergebnisse mit dem Lösungsblatt.

4. Beschreiben Sie anschließend die Sitten in Ihrem Land.

ja

nein

\begin{tabular}{|l|l|l|}
\cline { 3 - 3 } & Deutschland & \\
\hline$\square$ & Schweiz & \\
\hline$\square$ Österreich & & \\
\hline & & \\
\hline
\end{tabular}

1. Den Vornamen des Kindes bestimmen die Eltern.

2. Es gibt, im Verlauf der Jahre wechselnd, sehr beliebte, aber auch weniger beliebte Vornamen.

3. Vornamen können frei nach Phantasie gewählt werden.

4. Namen bestehen immer aus Vor- und Familiennamen.

5. Spitznamen und Kosenamen sind üblich.

6. Menschen- und Tiernamen sind manchmal identisch.

7. Die Frau übernimmt bei der Heirat den Namen des Mannes.
8. Namen können problemlos geändert werden, wenn sie nicht mehr gefallen.

9. Die Familiennamen werden an der Haustür angebracht.

10. Männliche und weibliche Namen sind immer unterschiedlich.

11. Am Namen ist die Schichtzugehörigkeit zu erkennen.

12. Es gibt spezielle Namenstage.

13. Das Kind bekommt seinen Vornamen einen Monat nach seiner Geburt.

14. Ein Name soll Glück bringen.

Verhalten - Rituale und Gewohnheiten

Abbildung 3: Wie soll das Kind denn heißen (aus: Hansen/Zuber 1996: 41) 
Expertengruppe ein Online-Modul entwickelt, das es Deutsch- und FranzösischLehrpersonen in den Niederlanden ermöglichen soll, selbständig eine systematische Lehrlinie (nl. »leerlijn«) für Landeskunde (bzw. Civilisation) in ihrem Unterricht $\mathrm{zu}$ entwickeln (vgl. https://www.internationalisering.nl/modules/ linq-landeskunde-civilisation/ bzw. Jentges 2018).

Letztlich kann nur mit Rücksicht auf Rahmenbedingungen der konkreten Unterrichtssituation und der jeweils verfolgten didaktischen Konzepte entschieden werden, welche Inhalte für die jeweilige Lerngruppe relevant sein können (vgl. auch Kapitel 2.2). Es kann nicht Ziel sein, die eine landes- und kulturkundliche Themenauswahl oder das eine allumfassende Lehrbuch für beliebige Zielgruppen weltweit zu formulieren bzw. zu erstellen. Es geht viel eher darum, ganz konkret vor Ort die jeweiligen Bedürfnisse und Möglichkeiten zu eruieren, wie Rösler bereits vor gut einem Vierteljahrhundert treffend formulierte:

[Es] braucht wahrscheinlich weniger große Modelle und noch integrativere Ansätze, sondern eher Modellversuche, anhand deren Beschreibung zukünftige Lehrer sehen können, wie wichtig es ist, die jeweiligen Individuen im Lernprozeß ernstzunehmen. Je mehr die Diskussion wegkommt von inhaltlichen und prozessuralen allgemeinen Vorgaben durch Didaktiker und Fachwissenschaftler mit ihren jeweiligen aktuellen Vorlieben und je stärker in der Lehreraus- und -fortbildung klar wird, daß landeskundliche Entscheidungen, egal ob implizit als Teil des Sprachlernprozesses oder explizit mit einer gewissen institutionellen Selbständigkeit, sinnvoll immer nur in Lernziel- und Zielgruppenabhängigkeit vor Ort getroffen werden können, desto weniger muß Landeskunde eine >unendliche Geschichte`, reine unlösbare Aufgabe ‘der gar das \Monster von Loch Ness der Fremdsprachenphilologie` (Picht 1989, 54) sein. (Rösler 1994: 71)

Es können kaum allgemeingültige Aussagen für die Auswahl von Inhalten und möglichen didaktischen Konzepten der Landes- und Kulturkundevermittlung im fremdsprachigen Unterricht für die jeweils individuelle Lern-/Lehrsituation zutreffend sein. ${ }^{8}$ Dazu kommt, dass der Gegenstand von Landes- und Kulturkunde - ob man ihn als Kultur im weitesten Sinne oder als die geographischen, ökonomischen und politischen Verhältnisse eines Landes sieht - sich ständig und mit jeder gesellschaftlichen Entwicklung verändert. Nahezu nichts in einer Gesellschaft bleibt längere Zeit konstant. Das Bild der deutschsprachigen Länder, das Lernende und auch Lehrende im Kopf haben, ist keineswegs einheitlich, sondern höchst differenziert und unterschiedlich. Die Bilder der deutschsprachigen Länder sind sowohl bei Lehrenden als auch Lernenden geprägt vom Wissen oder Vorwissen über das Land (z. B. häufig vermittelt durch Medien),

8 Vgl. Kapitel 2.2 zu regionalen und regionalisierten Lehrwerken, die viel stärker die jeweilige Zielgruppe und entsprechende Rahmenbedingungen vor Ort berücksichtigen können. 
von Erfahrungen in dem Land oder mit seinen Bewohnern und von Urteilen bzw. Vorurteilen über das Land. Auch Materialien, wie Lehrwerke und authentische Materialien aller Art sind nicht objektiv oder wahr, sondern je nach Autoren und Urheberschaft von unterschiedlichsten Auswahlkriterien geprägt (vgl. Zeuner 2009: 5).

Gerade diese kurz angerissenen Schwierigkeiten, Landeskunde überhaupt greifbar zu machen, können Landes- und Kulturkunde aber auch sehr spannend und vielleicht zu einem der interessantesten Teile des Fremdsprachenunterrichts machen (vgl. Zeuner 2009: 5), und, sicher zu einem Bereich des Fremdsprachenunterrichts, der didaktisch herausfordert und kreative Konzepte erfordert. Diese sollten jedoch vor allem abhängig von der jeweiligen Zielgruppe, ihren Lernzielen und den Rahmenbedingungen des jeweiligen Fremdsprachenunterrichts getroffen werden, und weniger zugunsten möglichst allgemeingültiger theoretischer Konzepte für eine allgemeine, für Lernende weltweit umfassende Landes- und Kulturkunde-Didaktik. Ähnlich wie Dietmar Rösler formuliert dann auch Uwe Koreik (2013: 182):

In einem modernen, in den Sprachunterricht integrierten Landeskundeunterricht wird auch in Zukunft folgendermaßen unterrichtet werden müssen: adressatenspezifisch und in Abhängigkeit von Lehrzielen und den jeweiligen Curricula sowie den damit verbundenen Prüfungen und Tests $[\ldots]$.

Immer entsteht eine Gratwanderung zwischen informierendem, kommunikativem und zur Lernerautonomie hinführendem, auf Interaktion setzendem und auf interkulturelle Lernziele hinarbeitenden Unterricht, dessen Schwerpunktsetzung (im Rahmen von institutionellen Vorgaben) maßgeblich von der jeweiligen Lehrkraft vorgenommen werden muss.

\subsection{Weitere Ansätze: Integrative und Erlebte Landeskunde}

Über die oben unter Abschnitt 3.1 beschriebenen, als traditionell zu bezeichnenden Ansätze zur Landeskunde-Vermittlung im Fremdsprachenunterricht und den im Abschnitt 3.2 speziell für den DaF-Unterricht umrissenen Forderungen hinsichtlich eines varietätsoffenen und pluritätssensiblen Unterrichts bezüglich der deutschsprachigen Länder hinaus, gab es bereits in den 1990er Jahren praktisch und theoretisch orientierte Impulse zu einer Innovation der Landes- und Kulturkundedidaktik im deutschsprachigen Raum. Zwei innovative Ansätze aus diesem Zeitraum, die auch das Aufgabenangebot im heutigen DaF-Unterricht weltweit bzw. den hierfür bereit gestellten Materialien weiterhin mitprägen, werden im Folgenden vorgestellt. 


\subsubsection{Das Tübinger Modell einer integrativen Landeskunde}

Mog konstatierte Anfang der 1990er Jahre hinsichtlich der Landeskunde-Debatte: »Im Sinne der ihr oft zugemuteten Totalität müßte sie [die Landeskunde] eigentlich das Fach aller erdenklichen Fächer sein, tatsächlich ist sie jedoch keine etablierte Disziplin, in den Wissenschaftsbetrieb nicht eingebunden und oft nur ein Appendix des Sprachunterrichts« (Mog 1992: 9).

Hiervon ausgehend unternahmen Paul Mog und Hans Joachim Althaus zu Beginn der 1990er Jahre den Versuch eines multidisziplinären Ansatzes zur Landeskundekonzeption im deutsch-US-amerikanischen Kontext, das so genannte »Tübinger Modell einer integrativen Landeskunde«. Herausgearbeitet werden sollte ein wissenschaftlich fundierter Ansatz für vergleichende Landesund Kulturkunde zwischen Deutschland und den USA. Kulturelle Traditionen und Profile anderer deutschsprachiger Länder wurden entsprechend nicht thematisiert, auch eine Darstellung zur ehemaligen DDR bzw. den hieraus möglicherweise resultierenden Unterschieden zwischen Ost- und Westdeutschland blieb aus. Ziel des Modells war es,

anhand ausgewählter Themen jenseits von elementarem Lern- und Merkwissen, von nur aktuellen Fragen, von binnendeutschen Spezialistendebatten und über einsinnige Fachperspektiven hinaus, Hintergründe und übergreifende Bezüge herzustellen. Die Darstellung eines solchen, bis in die feinsten Verästelungen des deutschen Habitus hineinreichenden geschichtlichen Lebenszusammenhangs ermöglicht wohl am ehesten das postulierte Verstehen der fremden und damit auch der eigenen Kultur. Vor allem diejenigen Kapitel, die sich mit Mentalitäten und Verhaltensweisen in sozialen Figurationen befassen, bleiben nicht beim sogenannten gesicherten Wissen stehen. Die Darstellung unternimmt interdisziplinäre Erkundungen und lädt damit ein zum produktiven Mit- und Weiterdenken; sie entspricht also der in den letzten Jahren vermehrt betonten Eigentätigkeit und der Prozeßhaftigkeit interkulturellen Lernens. Auch das TM [Tübinger Modell] verzieht die Wende zur Alltagskultur und zu einer verstärkten Erfahrungsorientierung, gelangt jedoch durch die Integration ganz unterschiedlicher Fachperspektiven (Geschichte, Kulturwissenschaft, Anthropologie, Soziologie, Politik, Germanistik) darüber hinaus. Kontroverse Konzepte werden auf diese Weise vermittelt und die Verabsolutierung einzelner Personen relativiert. (Mog 1992: 13)

Das Tübinger Modell wollte somit darauf reagieren, dass in der LandeskundeDebatte fächerübergreifend meist nur im Sinne von additiv und somit unverbunden gearbeitet wurde. Versuche, einen Kernbestand der für die Landeskunde einschlägigen Fächer festzulegen, verliefen weitgehend erfolglos: In der Landeskunde-Diskussion gerieten und geraten bei der zufälligen Themenauswahl von Alltagssegmenten historische sowie soziokulturelle Dimensionen oder die politischen und ökonomischen Strukturen eines Landes leicht aus dem Blick. Laut der Autoren haben sowohl die hohe Kultur als auch die Alltagskultur ihren 
legitimen Anspruch in der Landeskunde. Daher sollten beide "Kulturbegriffe» (kleines »k" und großes »K«) in ihr Modell integriert werden.

Unter integrativer Landeskunde wird im Tübinger Modell darüber hinaus und ist so explizit formuliert durchaus zum damaligen Zeitpunkt als innovativ zu sehen - die Integration von sprachlichem und (inter-)kulturellem Lernen verstanden, für die im das theoretische Modell begleitenden Praxisband Typisch deutsch? (Behal-Thomsen et al. 1993) zahlreiche anschauliche Beispiele präsentiert werden. Abbildung 4 zeigt ein Beispiel zur Sensibilisierung unterschiedlicher Bedeutungsvarianten der Begriffe "privat" und "öffentlich" im deutsch- und englischsprachigen, hier US-amerikanischen Kontext; Abbildung 5 Aufgabenvorschläge für die Erarbeitung des Begriffs »Heimat«.

Integrativ betont im Tübinger Modell zugleich auch den innovativen und wissenschaftlich fundierten Versuch, zentrale Themen der "Deutschlandkunde» in interdisziplinärer Kooperation verschiedener Fachdisziplinen zu beleuchten. Nicht additives Nebeneinander, sondern eine Kooperation unterschiedlicher Fachperspektiven (Geschichte, Kulturwissenschaft, Anthropologie, Soziologie, Politik, Germanistik) soll soziokulturelle Grundmuster für Themenstellungen und Lernziele der Landeskunde herausarbeiten, um so die Alltagskultur auf Basis des Hintergrunds der »hohen Kultur« darstellen zu können. Dieses u.a. kulturhistorische und soziokulturelle Hintergrundwissen wird ausführlich im theoretischen Band „Die Deutschen in ihrer Welt« vor allem für Lehrpersonen und ggf. sehr fortgeschrittene DaF-Lernende, z. B. im Universitätsstudium, aufbereitet. Letztendlich hat sich dieser - trotz aller Einschränkungen in der Umsetzung9 - als vorbildlich zu bezeichnende Versuch, landes- und kulturkundliche Inhalte wissenschaftlich fundiert und Sprach- sowie Inhalts- bzw. Kulturarbeit integriert anzubieten, auf theoretischer Modellebene nicht flächendeckend etablieren können. Die meisten der im Praxisband vorgestellten Aufgabenideen dürften jedoch auch heute noch, gut 25 Jahre nach dessen Erscheinen, für einen integrativen Sprach- und Landeskundeunterricht einsetzbar sein, und sind - trotz ihrer kontrastiven deutsch-englischsprachigen (USamerikanischen) Ausrichtung - mit kleinen Adaptionen auf andere Unterrichtskontexte übertragbar bzw. auch in neueren DaF-Lehrmaterialien als Ideenimpuls zurückzufinden.

Ein wesentlicher, von den Autoren des Tübinger Modells selbst genannter Schwachpunkt dürfte für alle Versuche von Bedeutung sein, die kulturkontrastives Vorgehen im Fremdsprachenunterricht umsetzen wollen: Man wollte

9 Die Autoren selbst geben an, dass es sich nicht um ein mustergültiges Modell für die Fächerauswahl der "Deutschlandkunde» handelt, in den vorgelegten Beschreibungen fehlen zentrale Bereiche wie Wirtschaft, Religion, Sport, Kunst und Technik; auch konnten geschlechtsspezifische Wahrnehmungen und Erfahrungen kaum berücksichtigt werden. 


\section{Wörter und Wendungen}

1. Was assoziieren Sie mit den englischen Wörtern private und public?
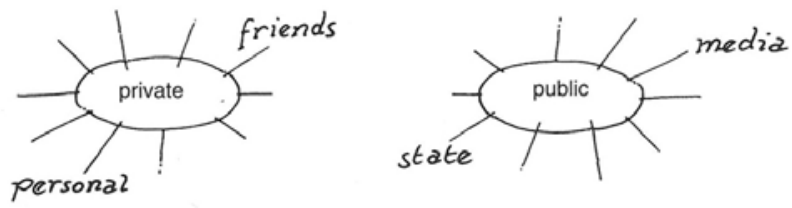

2. Vergleichen Sie bitte Ihre Assoziationen mit denen von anderen in der Gruppe. Diskutieren Sie miteinander über unterschiedliche Vorstellungen und Meinungen. Stellen Sie auch fest, ob bestimmte Assoziationen bei allen oder den meisten übereinstimmen.

3. Wortfelder private und public: Welche verwandten oder abgeleiteten englischen Wörter, Ausdrücke, Redewendungen etc. fallen Ihnen ein?

\begin{tabular}{|c|c|}
\hline private & public \\
\hline $\begin{array}{l}\text { privacy } \\
\text { in private }\end{array}$ & $\begin{array}{l}P R \\
\text { to publish }\end{array}$ \\
\hline$\cdots$ & $\cdots$ \\
\hline
\end{tabular}

4. Welche deutschen Übersetzungen finden Sie im Wörterbuch für private und public und die verwandten Wörter, Ausdrücke etc., die Sie in den Listen oben zusammengestellt haben? Für welche englischen Wörter und Wendungen gibt es im Deutschen genaue Entsprechungen, für welche nicht? Was z. B. finden Sie unter dem Stichwort privacy?

Privat

$$
\begin{aligned}
& \text { pri-vat Adj. auferasmolich, nicht öffentlich, } \\
& \text { persōnlich, vertraulidy nicht stantich (Ge- } \\
& \text { schäf, Unternehmen) - (Aufschrift auf Tar- }
\end{aligned}
$$

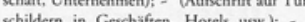

$$
\begin{aligned}
& \text { Angelegen } \\
& \text { Angelogenheiten; - er Eingang; das ist meine } \\
& \text { - } c \text { Meinung (dic ich nicht offentlich außere) } \\
& \text { - c Mitteilung, kann ich bitte Hernn X ein- } \\
& \text { mal - sprechen? [von lat. prinutus -(der Herr- } \\
& \text { schaft) beraubt, gesondert"; zu lat. privare } \\
& \text {,berauben, befreien, sondern"]. } \\
& \text { aus: Gerhard Wahrig. Deutsches Worterbuch. }
\end{aligned}
$$

$$
\begin{aligned}
& \text { privat „persönlich; vertraulich, fami- } \\
& \text { liär; nicht offentlich, außeramtlich": } \\
& \text { Im 18. Jh. aus lat. privătus „(der } \\
& \text { Herrschaft) beraubt; gesondert, furr } \\
& \text { sich stehend; nicht offentlich“ ent- } \\
& \text { lehnt, dem Partizipialadjektiv von lat. } \\
& \text { priväre „berauben; befreien; sondern". } \\
& \text { Stammwort ist lat. privus "für sich } \\
& \text { stehend, einzeln", das als Bestim- } \\
& \text { mungswort in } \rightarrow \text { Privileg erscheint.- } \\
& \text { aus: Duden, Etymologio, } \\
& \text { Bibliographisches Insttut, Mannheim } 1963
\end{aligned}
$$

Abbildung 4: Wortbedeutung "privat« und »öffentlich» (aus: Behal-Thomsen et al. 1993: 45) 


\section{Heimat}

1. Heimat - Welche englische Übersetzung finden Sie im Wörterbuch? Fallen Ihnen ähnliche Begriffe im Englischen ein, die Sie an dieser Stelle im Wörterbuch nicht gefunden haben? Verwandte, aber nicht deckungsgleiche Begriffe im Deutschen sind:

$$
\text { Heimat - Nation - Vaterland }
$$

Gelingt Ihnen eine Abgrenzung der Bedeutungen? Ziehen Sie gegebenenfalls ein Konversationslexikon zu Rate oder suchen Sie Belege oder Anwendungsbeispiele aus Zeitungen, Zeitschriften etc.

2. Durch die Lektüre der folgenden Textpassagen und die Interpretation der gezeigten Bilder können Sie sich der Definition des Begriffs nähern.

Schreiben Sie alle Schlüsselbegriffe, die zu einer Definition beitragen, in die rechten Spalten. Vergleichen und diskutieren Sie Ihre Ergebnisse in der Gruppe.

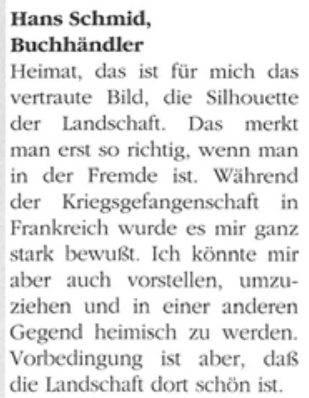

Unter Heimat verstehe ich den Ort, an dem ich aufgewachsen bin, Freunde und Bekannte habe. Das ist so ein Gefühl der Vertrautheit - etwa zu wissen, wo man einkaufen oder Spaziergänge machen kann. Heimat ist auch eng an die Sprache geknüpft. Ich könnte mir aber auch vorstellen, umzuziehen, neue Verbindungen herzustellen und mich einzuleben. Die Jugend ist nicht mehr so stark an ihren Geburtsort gebunden.

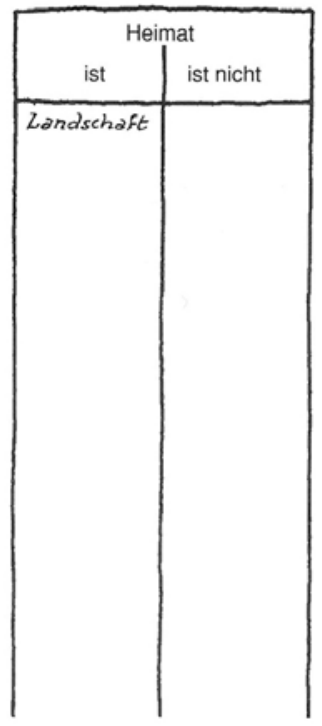

\section{6}

Abbildung 5: Wortbedeutung "Heimat« (aus: Behal-Thomsen et al. 1993: 126) 
Deutschland aus deutscher Sicht darstellen; gleichzeitig sollten aber auch amerikanische Deutschlandbilder und deutsche Amerikabilder einbezogen werden. Diese wurden jedoch durch notwendige Verkürzungen und Verallgemeinerungen zu klischeeanfällig: Amerikanische Deutschlandbilder entpuppten sich immer wieder als Homo- und Heterostereotype, nämlich als deutsche Vorstellungen von amerikanischen Deutschlandvorstellungen, wodurch die kontrastiven Einschübe nicht mehr amerikanische Realität, sondern nur die deutschen Bilder von ihr einfangen konnten (vgl. Mog 1992: 13f).

\subsubsection{Erlebte Landeskunde}

Im Diskurs zur Integration von Landeskunde im DaF-Unterricht etablierte sich Mitte der 1990er Jahre die Einsicht, dass sich die Kultur einer Gesellschaft am eindringlichsten durch direkte, kognitive sowie affektive Erfahrung derselben entdecken lässt, einer der wesentlichen Vorteile, die auch außerschulischen Lernorten zugesprochen werden (vgl. u. a. Gehring/Stinshoff 2010; BurwitzMelzer et al. 2015; Wrobel/Ott 2018). Insbesondere vom Goethe-Institut wurde diese Einsicht der "Erlebten Landeskunde" aufgegriffen und im Rahmen eines Fortbildungsprogramms, das Lehrpersonen, die außerhalb des deutschsprachigen Raums DaF unterrichteten, nach Deutschland brachte und sie durch projektähnliche Erkundungsaktivitäten Land und Leute erleben ließ, ausgebaut (vgl. Biechele/Meinert 2001). Hierbei sollten sie Erkenntnisse und Anregungen für den eigenen Unterricht gewinnen. Für die handlungsorientierte, erlebte Landeskunde spielten die räumlichen Gegebenheiten eine Schlüsselrolle; viele der Aktivitäten sind im thematischen Bereich der Stadterkundung angesiedelt und bringen die teilnehmenden Lehrpersonen dazu, alltagspraktische und kulturelle Besonderheiten der Stadt bzw. des urbanen Raums zu entdecken sowie gleichzeitig darüber zu reflektieren, dass Wahrnehmung subjektiv sowie kulturell- und vorwissensgeprägt erfolgt. Zahlreiche Aktivitäten gehen in diesem Sinne über die typischen Stadtrallyes hinaus, welche meistens mit Faktenfragen zum Abarbeiten funktionieren. So suchen die Teilnehmenden z. B. bei der "Stadterkundung mit offenen Sinnen" (Biechele/Meinert 2001: o. S.) nach Spuren eines selbst gewählten Begriffes, welcher für die Stadt charakteristisch sein könnte (z. B.: Wald für Boppard), und sollen herausfinden, ob sich in diesem Begriff wirtschaftliche, soziale oder kulturelle Aspekte der Stadt widerspiegeln (vgl. ebd.). Oder im Beispiel »Das besondere Detail» (vgl. Biechele/Meinert 2001: o. S. sowie Beispiel 1) bearbeiten sie Wahrnehmungsaufgaben zu einem Ort (Menschen, menschliches Verhalten, Geräusche etc.) und hinterfragen im Anschluss an die Präsentationen die beobachteten Situationen nach ihrer Kulturund/oder Ortsspezifik (ebd.). 


\begin{tabular}{|c|c|}
\hline Ziele & $\begin{array}{l}\text { - Einführen in die Projektarbeit } \\
\text { - Schärfen der Wahrnehmung von konkreten Alltagsdetails } \\
\text { - Reflektieren über deren landeskundlichen Bedeutung } \\
\text { - Konfrontieren mit kulturellen Bedeutungen in eigenen Ländern } \\
\text { - Erstes Kennenlernen des Seminarortes } \\
\text { - Orientieren in der unmittelbaren Umgebung des Seminarlokals }\end{array}$ \\
\hline
\end{tabular}

Günstige Stelle Am 1. oder 2. Seminartag und als 1. Schritt der Stadterkundung

Ablauf

Zeit Erkundung: 90 Minuten

Auswertung: pro Partnergruppe 10 Minuten

\section{Benötigtes Karten mit »besonderen Details«}

Material

Kommentar Die Aufforderung, ihr »Objekt« möglichst genau zu beschreiben, stellt hohe sprachliche Anforderungen. Der Akzent der Auswertung sollte aber nicht in erster Linie auf der Spracharbeit liegen, sondern auf dem, was den Teilnehmern aufgefallen ist und warum es ihnen aufgefallen ist, also auf der Vorbereitung der kontrastiven Landeskundearbeit. In der Regel stellt die Auswertung des »besonderen Details« eine Fundgrube für erste kontrastive Erfahrungen und authentische Materialien dar. Deswegen lässt sich die Erläuterung des Konzepts sehr gut an diese Auswertungsphase anschließen.

Statt Beobachtungsaufgaben kann man auch ausschließlich Höraufgaben stellen, wie z. B. »Geht bitt zum Mariannenplatz, legt euch ins Gras oder setzt euch auf eine Bank, schließt die Augen. Wie klingt der Platz?« (Bahnhof Zoo, Café Möhring, Mensa U-Bahn, Parkanlage usw.)

Beispiel 1: Das besondere Detail (aus: Biechele/Meinert 2001: o. S.)

Dieser handlungsorientierte Ansatz zur serlebten Stadterkundung`, der häufig persönliche Erfahrungen, Wahrnehmung und Reflexion dieser - inkl. Sinneswahrnehmungen - in den Mittelpunkt stellt, bietet heute, im digitalen 21. Jahrhundert, viele unterrichtspraktische Ideen, die auch als Online-Projektaufgaben oder im digitalen sowie realen Austausch für den Bereich der 
Landes- und Kulturerkundung ein- bzw. umgesetzt werden können. In Anlehnung an die oben gemachten Ausführungen, Entscheidungen für die Auswahl landes- und kulturkundlicher Inhalte (vgl. auch Absatz 3.3, Kapitel Lehrmaterialanalyse 4.2 und 4.3) immer vor allem Zielgruppen- und Lernzielabhängig entsprechend der jeweiligen Rahmenbedingungen vor Ort zu treffen, werden im Folgenden weitere Entwicklungen und Vorschläge zum landes- und kulturkundlichen Lernen aus dem 21. Jahrhundert präsentiert.

\subsection{Interkulturelles Lernen}

Interkulturelles Lernen zielt - auf die eine oder andere Weise - auf die Förderung interkultureller Kompetenz. Deardoff (2006:5) versteht interkulturelle Kompetenz als Fähigkeit, „auf Grundlage bestimmter Haltungen und Einstellungen sowie besonderer Handlungs- und Reflexionsfähigkeiten in interkulturellen Situationen effektiv und angemessen zu interagieren«. Zur Beschreibung und Entwicklung interkultureller Kompetenz liegen zahlreiche Modelle vor (vgl. u. a. Rathje 2006; Bolten 2007; Over et al. 2008). Und - zumindest in Deutschland - können interkulturelle Lerninhalte und Arbeitsformen sowie internationale Begegnungen zum festen Bestandteil in Curricula und Lehrplänen fast aller Fächer und Bildungsbereiche - vom Kindergarten bis zur Erwachsenenbildung - gezählt werden. Das Konstrukt des interkulturellen Lernens umfasst unterschiedliche Teilkompetenzen und methodisch-didaktische Prinzipien, zu denen zählen:

- die Fähigkeit, sich mit Fremdheit reflexiv auseinanderzusetzen,

- dabei von der Gleichwertigkeit aller Kulturen und der Relativität der eigenen Kultur auszugehen,

- Angehörigen fremder Kulturen nicht das eigene Modell aufzunötigen, sondern sich der fremden Kultur mit Empathie und der Bereitschaft zur Perspektivenübernahme zu nähern und dabei sich durch Fremdes auch in Frage stellen zu lassen (vgl. Nolda 1995: 94).

Insbesondere die in den 1990er Jahren entstandenen Lehrwerke Sichtwechsel und Elemente sowie die Materialsammlungen zum landeskundlichen Lernen im DaF-Unterricht, Zwischen den Kulturen (Hansen/Zuber 1996) und Spielarten (Lundquist-Mog 1996), beinhalten ansprechende Beispielaufgaben, die Möglichkeiten zur Umsetzung einzelner Teilschritte interkulturellen Lernens aufzeigen. ${ }^{10}$ Die in Abbildung 6 und 7 gezeigten Aufgabenimpulse können dazu

10 Für eine ausführliche Analyse der Konzeption einer »Didaktik des Fremdverstehens« bzw. der didaktisch-methodischen Umsetzung von Teilschritten zum interkulturellen Lernen in den genannten Lehrwerken und Materialienbänden siehe Richter 1998. 
anregen, sich mit Fremdheit oder Andersartigkeit der Zielsprachenkultur(en) auseinanderzusetzen, wobei sich automatisch die Frage stellt, was für den einzelnen Lerner selbst üblich bzw. gewohnt ist, und liefern Gesprächsimpulse, ohne dabei Normen über die Zielsprachenkulturen vorzugeben.

Während sich das Beispiel aus Zwischen den Kulturen (Abbildung 6) international an Deutschlernende richtet und kulturunabhängig zum Nachdenken anregt, was im alltäglichen Leben für den einen sehr wohl, für den anderen aber eben nicht als üblich gilt, und auf den folgenden Seiten im Band auch eine Einschätzung dazu gibt, womit in den deutschsprachigen Ländern gerechnet werden kann (Stand Mitte der 1990er Jahre), bezieht sich Spielarten (Abbildung 7) auf einen deutsch-polnischen Lernprozess. Um eine Sensibilisierung von unterschiedlichen Wahrnehmungen zu initiieren, wird hier zunächst von Aussagen über Deutschland und Deutsche ausgegangen, die weder vermeintlich polnische noch deutsche Perspektive widerspiegeln.

Während mit den oben vorgestellten Aufgabenbeispielen bereits ein erster Schritt hinsichtlich Relativierung der eigenen kulturgeprägten Wahrnehmungen angeregt werden kann, setzen die in Abbildung 8 und 9 präsentierten Aufgabenbeispiele vor allem darauf, für einen selbst selbstverständliche Handlungsmuster und Wahrnehmungen/Empfindungen zu hinterfragen, auch hier wird wieder einmal kulturunabhängig und einmal kulturkontrastiv vorgegangen. 


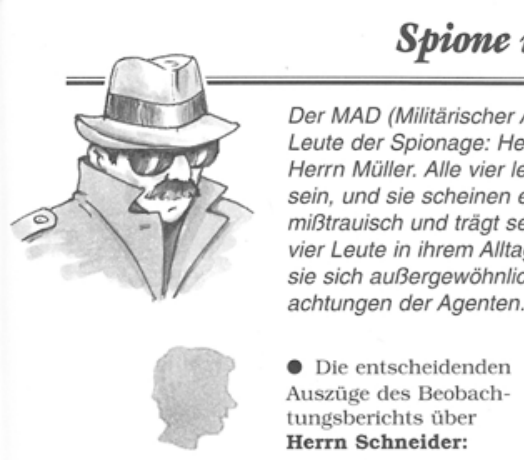

1. Herr Schneider läßt sich zum Bahnhof fahren und versucht, mit Reiseschecks zu bezahlen.

2. Wenn er irgendwo eingeladen ist, bringt er der Gastgeberin immer Blumen mit.

3. Sonntags nach dem Mittagessen mäht er mit dem elektrischen Rasenmăher den Rasen im Garten.

4. Er fragt im Rathaus im ersten Stock nach dem Ausgang.

5. Er trinkt regelmäßig Wasser zum Essen.

6. Er meldet sich am Telefon immer mit "Ja bitte?"

Spion: ja $\square \quad$ nein $\square$

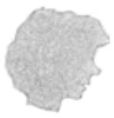

- Die entscheidender Auszüge des Beobachtundsberichts über Frau Lehmann:

1. Frau Lehmann sitzt mit Vorliebe in Cafés und behält dabei immer ihren Hut auf.

2. Wenn sie jemanden zur Mittagszeit in der Firma trifft, sagt sie „Mahlzeit”.

3. Manchmal bezahlt sie ihre Rechnungen in der Bank mit "Öberweisungen".

4. Am 1. Mai ist sie umgezogen. Am 7. meldet sie sich im Polizeirevier um.

5. Vor Benutzung einer U-Bahn oder eines Busses kauft sie nie eine Fahrkarte.

6. Beim Schuhkauf gibt sie die Größe $39^{1 / 2}$ an.

Spionin: $\quad$ ja $\square$ nein $\square$
1. Wenn Frau Meier in ihrem Haus Putzdienst hat, wischt sie den Hausflur und fegt den Bürgersteig.

2. Sie geht am Samstag nachmittag gegen $17^{\circ} \mathrm{Uhr}$ zum Supermarkt einkaufen.

3. Auf einem Kurzurlaub sucht sie eine preiswerte Unterkunft und steigt in einer Pension ab.

4. In einer Drogerie reicht sie ein Rezept von ihrem Arzt ein.

5. Am Montag, dem 13. Juni, steht sie vor dem Eingang des Historischen Museums.

6. Beim Abendessen im Gastraum wünscht sie ihrem Nachbarn „Großen Appetit!“

$$
\text { Spionin: } \quad \text { ja } \square \text { nein } \square
$$

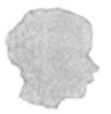

- Die entscheidenden Auszüge des Beobachtungsberichts über Herrn Müller:

1. Herr Mūller sagt immer „Gesundheit”, wenn jemand niest.

2. Etwa einmal im Monat geht er zur Post, um Geld abzuheben.

3. Er geht nie bei Rot über die Straße.

4. In einem fremden Wirtshaus setzt er sich an einen Tisch, auf dem „Stammtisch“ steht.

5. Am Montag, dem 5. April, geht er zum Friseurladen "Schnelle Schere“.

6. Am 8. Juni lāßt er im Cafẻ „Burkhardt" ein Formular_Arbeitserlaubnis" liegen.

$\begin{array}{ll}\text { Spion: } & \text { ja } \square \quad \text { nein } \square \\ & \text { Verhalten - Im Alltag }\end{array}$

Abbildung 6: Reflexive Auseinandersetzung mit »Fremden«: Spione unter uns (aus: Hansen/ Zuber 1996: 35) 


\section{Deutsche und Polen - Cegenseifige Wahrnehmung}

\section{Übersicht}

Das Kapitel soll den Lernenden zur Erkenntnis verhelfen, daß eine Aussage über ein fremdes Land (in dem Fall Deutschland) nicht zuletzt auch von der Heimatkultur des/der Sprechenden oder Schreibenden geprägt wird. Sie sollten sich der eigenen Stereotypen über Deutschland bewußt werden und über den Wahrheitsgehalt von Stereotypen und deren Relativierung nachdenken.

Dann geht es um den wechselseifigen Einfluß beider Länder auf den verschiedenen Ebenen von Geschichte, Sprache und Kultur, wobei Verbindendes und Trennendes zwischen Polen und Deutschland erkennbar werden soll.

Das Kapitel führt schließlich zu der Aufgabe, selbst Möglichkeiten zu finden, die zu einem besseren Verständnis zwischen beiden Ländern verhelfen können.

\section{Fremdwahrnehmung}

1 Lesen Sie die folgenden Aussagen über Deutschland und die Deutschen. Erraten Sie das Herkunftsland des Sprechers/der Sprecherin. Ist es Japan, Griechenland oder sind es die USA?

W USA

A. Ja, die Disziplin, die die Kinder schon lernen. Deutsche Kinder (1) (chlafen gehen. Und um sieben Stunde um sieben Uhr Sandmännchen angucken unde kinder erst mal eine halbe stig im still sein. Dann lassen sie dufrieden, weil das Kind rech muß alles still sein. dann sind sie Bett gewesen ist." "Hier in Deutschunterzuschmeißen. Bei uns ist das anders; man denkt, auf eine Schachtel zigaretten kommt es auch nicht drauf an."

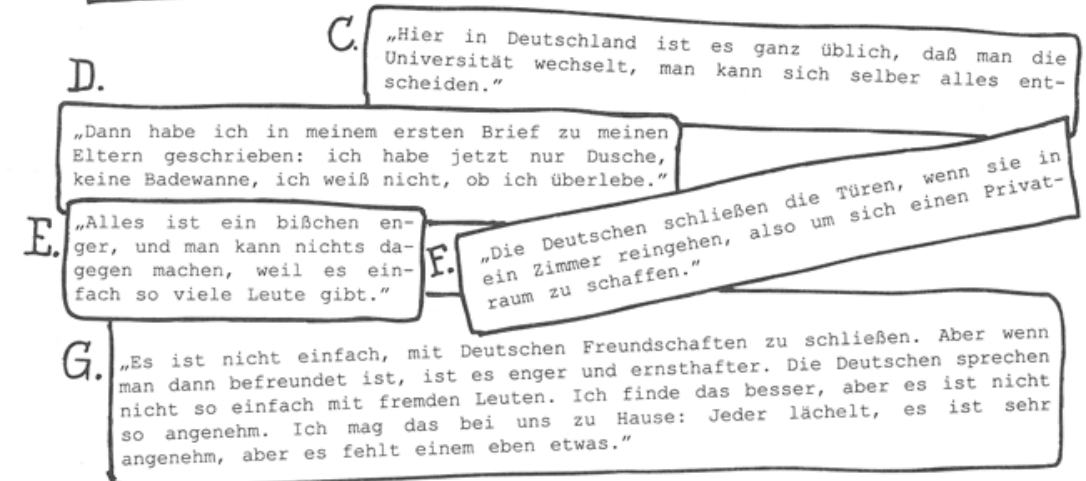

2 Warum kann man in einigen Fällen von den Aussagen direkt auf das Herkunftsland schließen?

\section{4}

Abbildung 7: Reflexive Auseinandersetzung mit »Fremden«: Gegenseitige Wahrnehmung (aus: Lundquist-Mog 1996: 14) 
Versetzen Sie sich in Ihrer Kleingruppe in die unten angegebenen Situationen und stellen Sie jeweils fest, ob Ihnen in der jeweiligen Situation das Warten nichts ausmacht, oder benennen Sie den Zeitpunkt, ab dem Sie anfangen, ungeduldig zu werden. Wie bewerten Sie dann die Situation, sich selbst oder die anderen? Machen Sie Notizen in den dafür vorgesehenen Rubriken.

\section{Wartesituation}

Beispiel: Beim Arzt

1. Der Zug hat Verspätung, und Sie sitzen drin.

2. Sie erwarten Besuch: zum Essen;

zu einem Fest.

3. Sie sind in einem Lokal verabredet:

mit dem Lebenspartner/ der Lebenspartnerin; mit einem guten Freund/ einer guten Freundin; mit einem entfernten Bekannten.

4. Vor einem Jobinterview: Sie sitzen im Vorraum.

5. Sie haben einen beruflichen Termin im Büro Ihres/r Geschäftspartners/in, der/die Sie warten läßt.

6. Sie wollen in den Urlaub fliegen, und das Flugzeug hat Verspätung.

7. Sie haben einen Termin beim Steuerberater.

8. Sie sind an einem Treffpunkt draußen verabredet, und inr/e Partner/in läßt Sie warten.

9. Sie stehen in einer langen Schlange beim Einkaufen.

10. Sie kommen an der Bushaltestelle an, und der Bus ist gerade abgefahren.

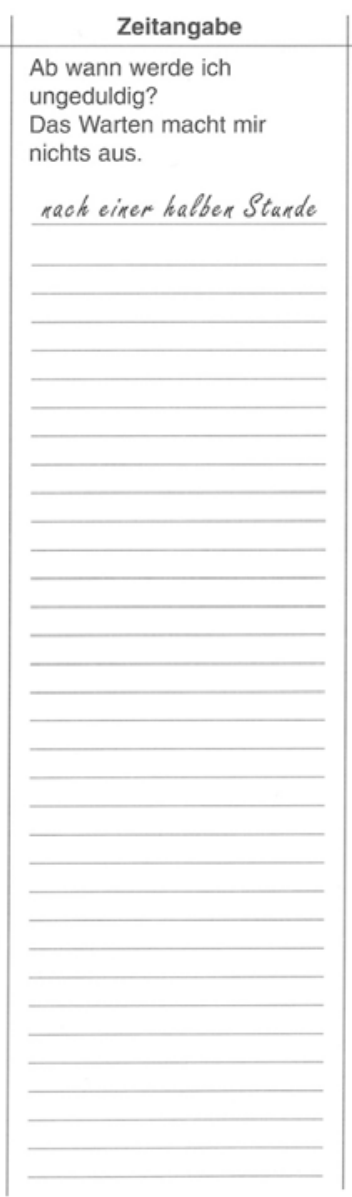

\section{Bewertung}

Wie bewerte ich dann meinen Kommunikationspartner, die Situation und mich selber?

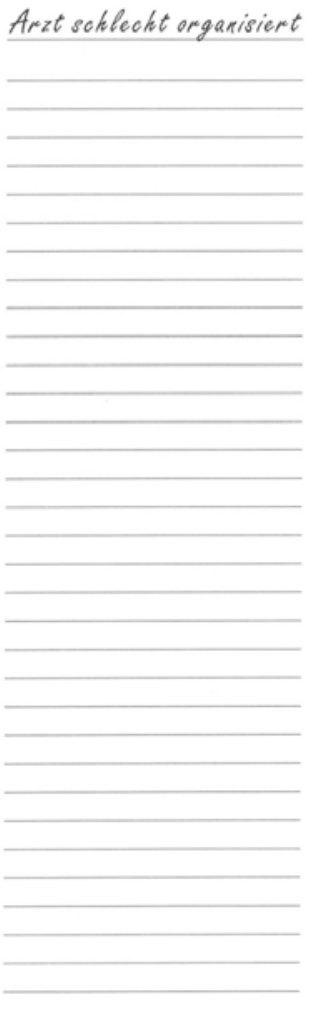

Verhalten - im Alltag

Abbildung 8: Relativierung der »eigenen« Kultur: Wertvolle Zeit (aus: Hansen/Zuber 1996: 39) 


\section{Deutsche und Polen - Gegenseifige Wohmehmung}

\section{Typische Eigenschaften?}

1

a Alle Gruppenmitglieder schreiben auf 4 gleichaussehende Kärtchen:

- eine positive deutsche Eigenschaft

- eine negative deutsche Eigenschaft

- eine positive polnische Eigenschaft

- eine negative polnische Eigenschaft

Die Kärtchen werden anschließend gemischt und neu verteilt. Versuchen Sie nun gemeinsam, die jeweilige Eigenschaft einer der vier Kategorien zuzuordnen.

b Sind Sie immer der gleichen Meinung? Welche Eigenschoften haben Sie als Gruppe einstimmig einer Kategorie zugeordnet? Hätten Sie nicht auch die Eigenschaften anders zuordnen können? Versuchen Sie, auch die scheinbar eindeutigen Eigenschaften im Gespräch differenzierend zu betrachten.

Wenn Sie eine formalisierte Diskussion führen wollen, benutzen Sie die Redemittel und Argumentationsmuster aus dem Köstchen.

\begin{tabular}{|c|c|c|}
\hline Begründen: & Bezweifeln: & Differenzieren: \\
\hline $\begin{array}{l}\text { - darlegen } \\
\text { - herausheben } \\
\text { - sich beziehen ouf } \\
\text { - hinweisen auf } \\
\text { - feststellen } \\
\text { - folgern } \\
\text { - verweisen }\end{array}$ & $\begin{array}{l}\text { - widersprechen } \\
\text { - einwenden } \\
\text { - einschränken } \\
\text { - bestreiten } \\
\text { - Diese Meinung ist nicht haltbar, } \\
\text { du übersiehst dabei ... } \\
\text { - ... scheint mir problematisch. } \\
\text { - Das muß ich in Frage stellen. } \\
\text { - Das sehe ich ganz anders. } \\
\text { - Ich bitte zu bedenken, daß ... }\end{array}$ & $\begin{array}{l}\text { - In einzelnen Punkten kann } \\
\text { ich dir zustimmen, aber ... } \\
\text { - Einerseits kann ich dir bei- } \\
\text { pflichten, daß ... } \\
\text { - Zwar hast du recht, wenn } \\
\text { du das betonst, aber ... } \\
\text { - Zu dem, was du sagst, } \\
\text { müBten noch ganz andere } \\
\text { Aspekte herangezogen } \\
\text { werden, nämlich ... }\end{array}$ \\
\hline
\end{tabular}

Abbildung 9: Relativierung der »eigenen« Kultur: Typische Eigenschaften? (aus: LundquistMog 1996: 15) 


\subsubsection{Interkulturelles vs. kulturreflexives Lernen}

Die den Konzepten zum interkulturellen Lernen zu Grunde liegende Dichotomie von "Eigenem" und "Fremdem" wird zusehends als problematisch gesehen, scheint sie doch davon auszugehen, dass Individuen $\mathrm{zu}$ deutlich voneinander abgrenzbaren Kulturgemeinschaften zuzuordnen wären und eben die Gegenüberstellung von »Eigenem» und »Fremden« zu kurz greift, um heutigen gesellschaftlichen Realitäten gerecht zu werden (vgl. u.a. Altmayer 2008: 28ff.; Rössler 2010: 140). Letztlich liegt einer interkulturellen Landeskunde häufig ein homogener Kulturbegriff zu Grunde, wie dieser u. a. von Alexander Thomas, der Kultur als ein universelles, für eine Gesellschaft sehr typisches Orientierungssystem sieht, in seinem Kulturstandard-Ansatz beschrieben wurde:

[Kulturstandards] sind alle Arten des Wahrnehmens, Denkens, Wertens und Handelns [...], die von einer Mehrzahl der Mitglieder einer bestimmten Kultur für sich persönlich und andere als normal, selbstverständlich, typisch und verbindlich angesehen werden. Eigenes und fremdes Verhalten wird auf der Grundlage dieser Kulturstandards beurteilt und reguliert.

[...] Dieses Orientierungssystem wird aus spezifischen Symbolen gebildet und in der jeweiligen Gesellschaft usw. tradiert. Es beeinflußt das Wahrnehmen, Denken, Werten und Handeln aller ihrer Mitglieder und definiert somit deren Zugehörigkeit zur Gesellschaft. Kultur als Orientierungssystem strukturiert ein für die sich der Gesellschaft zugehörig fühlenden Individuen spezifisches Handlungsfeld und schafft damit die Voraussetzung zur Entwicklung eigenständiger Formen der Umweltbewältigung. (Thomas 1993: 381)

Kultur-Konstruktionen in diesem Sinne repräsentieren eine Vorstellung von Kultur als homogene, in sich abgeschlossene Einheiten, und müssen zwangsläufig zu stark verallgemeinernden Aussagen kommen (vgl. Adamczak-Krysztofowicz et al. 2015: 14). Laut Altmayer kann ein solches Kulturkonzept nicht nur zu einer Verfestigung von Stereotypen führen, sondern ist »geradezu schädlich« (Altmayer 2004: 103).

Davon ausgehend, dass heutzutage »Kulturen nicht als homogene Entitäten zu fassen sind, sondern sich durch Heterogenität und Pluralität auszeichnen, [...] sie nicht statisch zu denken [sind und] [...] der Kulturbegriff ein breites Spektrum lebensweltlicher Phänomene umfasst « (Rössler 2010: 138) wird die Adäquatheit des Begriffs »interkulturell« tatsächlich fragwürdig, denn »wie sähe eine Begegnung inter, d.h. zwischen den »Kulturen« aus, wenn Kulturen selbst heterogen und dynamisch sind? Was wäre eigen, was fremd? (Hoch 2016: 16). Um die Begegnung zwischen Kulturen zu betonen, erscheint in den letzten Jahren des Öfteren der Begriff transkulturell (siehe u. a. Wormer 2004, 2006 und Absatz 3.5.2). Das Präfix "trans« soll darauf hinweisen, dass die Landeskunde Begegnung mit anderen Kulturen anstrebt und dabei immer von der »eigen- 
kulturellen Prägung« ausgeht. Auch Schweiger et al. (2015: 4) fordern ein komplexeres, dynamischeres Verständnis von Kultur, "als es die Unterscheidung zwischen Eigen- und Fremdkultur nahelegt«, nämlich einen kulturwissenschaftlichen Landeskundeansatz (vgl. Absatz 3.5.2), dessen Kern (Kultur-)Reflexivität bietet, für die »[d]ie kritische Beschäftigung mit dem alltäglichen sowie wissenschaftlichen Verständnis von Kultur [...] für eine reflexive Landeskunde zentral [ist] « (Schweiger et al. 2015: 10). Kulturreflexive Ansätze würden somit die vermeintlichen Zielsprachenkulturen nicht nur thematisieren und mit der vermeintlich eigenen Kultur kontrastieren, sondern diese diskutieren.

\subsubsection{Diskursive Landeskunde}

Aktuelle kulturwissenschaftliche Ansätze propagieren die oben bereits beschriebene Orientierung an Kulturreflexion ${ }^{11}$, im Sinne von Kultur als heterogene Deutungsmuster und deren Diskursivität. Als repräsentativer neuerer Versuch einer theoretischen Fundierung von Landes- und Kulturkunde ${ }^{12}$ dürfte im deutschsprachigen Raum vor allem das inzwischen unter dem Begriff »Diskursive Landeskunde« bekannte Modell von Altmayer zu sehen sein.

Davon ausgehend, dass sich Inhalte und Ziele des landeskundlichen Lernens stetig verändern und entsprechend immer wieder diskursiv ausgehandelt werden müssen, versteht Altmayer Kultur als ein heterogenes, vielschichtiges Konstrukt, einen Hypertext, der durch tradierte Muster der Welt- und Wirklichkeitsdeutung zum Ausdruck kommt (vgl. Altmayer u. a. 2002, 2004, 2006, 2007, 2008). Sprache spielt laut Altmayer hierbei eine wesentliche Rolle, da Landeskunde eng mit Sprache zusammenhängt und nur mit und im Sprachgebrauch, in Diskursen, zustande kommt.

>Kulturelles Lernen` findet laut Altmayer dann statt,

11 Für eine kritische Auseinandersetzung mit aktuellen Landes- und Kulturkundekonzepten vgl. u. a. Koreik 2018; Fornoff/Koreik (im Dr.); Fornoff/Koreik (in Vorbereitung).

12 Ein anderes Theorie-Modell wird im Ansatz von Wormer (2004 sowie 2006) vorgestellt, die hier beschriebene transkulturelle und vergleichende Landeskunde möchte sozialwissenschaftliche Erkenntnisse, d.h. empirische Methoden, in die Philologien bringen. Wormer definiert seine transkulturelle vergleichende Landeskunde als »transdisziplinäre, pluriperspektivische Tätigkeit, die in der Lebenswirklichkeit individuellen und institutionellen Handelns ihren Ausgang nimmt und kultur(en)kooperativ eine doppelt integrierende Perspektive einnimmt: das Integrieren sowohl verschiedener Disziplinen als auch verschiedener Kulturen." (Wormer 2006), im Sinne einer Reflexion der eigen- und fremdkulturbedingten Perspektive. Auf Wormers Theoriemodell wird im Folgenden nicht eingegangen, da keine unterrichtsbezogenen Fallstudien oder entsprechende Materialkonzepte vorliegen. 
[...], wenn Lerner [...] veranlasst durch die Auseinandersetzung mit deutschsprachigen >Texten anpassen, umstrukturieren, verändern oder weiterentwickeln, dass sie den kulturellen Deutungsmustern, von denen die Texte Gebrauch machen, weitgehend entsprechen und die Lerner in die Lage versetzen, diesen Texten einen kulturell angemessenen Sinn zuschreiben und dazu angemessen (kritisch oder affirmativ) Stellung nehmen können. (Altmayer 2007: 17f.)

In seiner Habilitationsschrift zeigt Altmayer (2004) am Beispiel einer Fallstudie zu »Ausländer, Deutsch, Integration«, wie kulturelle Deutungsmuster für das Fach Deutsch als Fremdsprache explizit und sichtbar und entsprechend für Mitglieder anderer Kommunikationsgemeinschaften nachvollziehbar gemacht werden können. So interessant diese Fallstudie ist, so komplex erweist sich die interpretativ sehr aufwändige Analyse von zahlreichen Text- und Bildmaterialien; eine Analyse, die von Lehrenden und Lernenden nur bei großem Vorwissen und kompetentem Sprachniveau zu leisten ist. Ansatz und Vorgehen mögen für die Aus- und Fortbildung von Fremdsprachen-Lehrpersonen vorbildlich sein; in der fremdsprachlichen Unterrichtsrealität, z. B. an Schulen auf Anfängerniveau, dürften sie jedoch kaum umsetzbar sein. Dass die Anwendbarkeit der Theorie in der Unterrichtspraxis stark einbüßt, war Altmayer selbst durchaus bewusst: »Kulturwissenschaftliches Wissen in dem Sinn, wie wir es hier entwickelt haben, ist eine zwar notwendige, aber nicht hinreichende Bedingung für die wissenschaftliche Aufwertung der herkömmlichen Landeskunde in der Praxis« (Altmayer 2004: 460).

Das Desiderat nach Anwendungsbeispielen eines aktuellen kulturwissenschaftlichen Ansatzes für den DaF-Unterricht wurde mit der Publikation des Materialienbandes Mitreden - Diskursive Landeskunde für Deutsch als Fremdund Zweitsprache ${ }^{13}$ (Altmayer 2016), die Altmayer selbst herausgegeben hat, eingelöst. Der Band gliedert sich in drei Module (Menschen, Essen und Mobilität), die jeweils auf einem unterschiedlichen Sprachniveaus (A2-B2) einsetzbar sind und Diskursfähigkeit fördern wollen. In der ausführlichen, theoretischen Einleitung zum Band wird erläutert, dass Diskurs als sozialer Prozess gesehen wird, durch den soziale Sinnzusammenhänge entstehen (vgl. Altmayer et al. 2017: 8): Diskurs also im Sinne von Bedeutungszuschreibung in Kommunikation. Davon ausgehend, dass als kognitive Schemata der Welt- und Wirklichkeitsdeutung beschriebene kulturelle Muster in Diskursen ausgehandelt werden, gilt als Lernziel Diskursfähigkeit. Diese wird als Alternative zu übergreifenden fremdsprachlichen Lernzielorientierungen wie kommunikative oder

13 Eine ausführliche Beschreibung und Analyse des Bandes im Sinne einer Umsetzung von Materialien für einen diskursiven vs. interkulturellen Landeskundeansatz bietet Zeuner (2017). 
interkulturelle Kompetenz gesehen und wird in diesem Ansatz in drei sprachbezogene Kernkompetenzen untergliedert:

- »die Fähigkeit von Fremdsprachenlernenden, an Bedeutungskonstruktionen in der Fremdsprache mitwirken, diese erweitern und hinterfragen zu können,

- die Fähigkeit, Diskurspluralität, d.h. das Neben- und Gegeneinander unterschiedlicher Positionen, Perspektiven und Meinungen im Diskurs, anzuerkennen und auszuhalten, und

- die Fähigkeit, die Praktiken der Bedeutungsproduktion im Diskurs zu durchschauen« (Altmayer et al. o. J.: 15).

Mit anderen Worten: Der Fremdsprachenlernende macht sich mit dem »fremden « Diskurs bekannt, durchschaut ihn und kann die eigene und fremde Position bzw. Perspektive im Diskurs kritisch reflektieren.

Um dies zu erreichen, werden folgende methodische Prinzipien einer Diskursiven Landeskunde genannt (vgl. Altmayer et al. 2017: 16f.):

- Authentische und vielseitige Texte

- Multiperspektivität der Texte

- Vorwissen und Erfahrungen der Lernenden ernst nehmen

- Problemorientierung und Reflexion über eigene und andere kulturelle Muster

- Vielfältige Sozialformen und »offene« Methoden (offen für subjektive Bedeutungskonstruktionen).

Zur Illustrationen der Umsetzung dieses zunächst durchaus komplex wirkenden Ansatzes - auch für den Einsatz im (fortgeschrittenen) Anfängerunterricht - sei auf die in Abbildung 10 dargestellte Aufgabenstellung verwiesen, die sich auch leicht für andere Themenbereiche umsetzen lässt.

Dennoch sollte angemerkt werden, dass das im Rahmen dieses Ansatzes formulierte, durchaus erstrebenswerte Ziel, dass anhand »authentischer Diskurse [...] die Fähigkeit der Lernenden gefördert werden [soll], die diskursive Konstruktion von Sinn und Bedeutung zu durchschauen, verschiedene Sichtweisen auszuhalten und schließlich an deutschsprachigen Diskursen des öffentlichen Lebens teilzuhaben« (Altmayer 2013: 108), kaum der Realität in den allermeisten Fällen des schulischen DaF-Unterrichts weltweit gerecht werden dürfte und in solchen auch höchstens punktuell mit Aufgabenbeispielen wie in Abbildung 10 eingelöst werden kann. ${ }^{14}$ Ein globaler Einsatz dieses Kulturvermittlungsansatzes ist zweifelsohne wünschenswert, dürfte aber entsprechend

14 Darüber hinaus sei mit Koreik (2018: 43) darauf hingewiesen, dass Diskurs nur mit einem gewissen Faktenwissen ermöglicht werden kann: »Es klingt sehr gut: Keine Fakten, sondern Diskurs! Nicht Wissen, sondern Fähigkeit zur diskursiven Auseinandersetzung! Aber ein Diskurs setzt zunächst einmal einen gewissen Kenntnisstand, die Fähigkeit zur Fragestellung setzt auch Wissen voraus." 


\section{Typisch Mann, typisch Frau?}

1a. Was ist ,typisch Mann', was ist, typisch Frau'? Ordnen Sie zu. Manche Aussagen passen mehrfach.

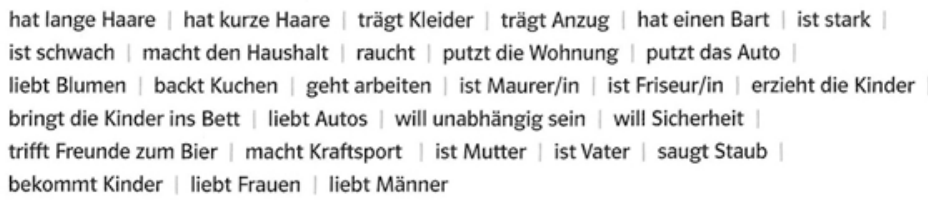

\begin{tabular}{|c|c|c|}
\hline & MANN & FRAU \\
\hline Körper / Aussehen & trägt Anzug & \\
\hline \multicolumn{3}{|l|}{ Charakter } \\
\hline Aufgaben & & ist Mutter \\
\hline \multicolumn{3}{|l|}{ Sonstiges } \\
\hline & & \\
\hline & & \\
\hline & & \\
\hline
\end{tabular}

1b. Vergleichen Sie Ihre Ergebnisse im Kurs. Sind Sie alle einer Meinung? Diskutieren Sie.

Abbildung 10: Typisch Mann, typisch Frau? (aus: Altmayer 2016: 22) 
der realen Rahmenbedingungen vor Ort, in der jeweiligen schulischen Lernsituation, häufig nicht möglich sein, wie auch Fornoff/Koreik (im Druck) konstatieren: "Es scheitert [u.a.] am mehrheitlich erreichten zu niedrigen Sprachniveau, es scheitert am oft von Lernenden nicht ausreichend mitgebrachten Weltwissen. Und es scheitert nicht selten auch an der durch in Heimatländern nicht vermittelten allgemeinen Diskurskompetenz«.

\subsubsection{Spatial turn: »Räume sagen mehr als tausend Worte«}

Ein weiterer Trend, der kulturwissenschaftliche Entwicklungen für den Fremdsprachenunterricht nutzbar machen will, ist in Konzepten zu sehen, die im Zeichen des so genannten spatial turns stehen, der seit Jahren beispielweise in der kulturwissenschaftlich orientierten Germanistik die Diskussion über Räumlichkeit und Raumwahrnehmung zentral stellt (vgl. Bachmann-Medick 2006; Delhey/ Jentges 2013: 179ff.; Cerri et al. 2015: 3ff.). Zu denken ist hierbei an neuere Konzepte aus den Kulturwissenschaften wie Erinnerungsorte, urban palimpseste, urban space, linguistic landscaping, die - wenn auch noch zögerlich - aber doch immer häufiger für den Fremdsprachenunterricht genutzt werden.

Ausgehend von theoretischen Raumkonzeptionen (vgl. hierzu u.a. Delhey/ Jentges 2013; Cerri/Dausend 2015) werden Möglichkeiten zu ihrer didaktischen und methodischen Umsetzung im Unterricht entwickelt. Diese Raumtheorien verstehen Raum und somit Kultur nicht nur als mit Zahlen und Fakten objektiv erfassbare Größe, sondern in verschiedenen Perspektiven differenziert, die auch eine subjektiv-konstruktivistische Wahrnehmung berücksichtigen. Für den Bereich des Fremdsprachenunterrichts, insbesondere des Deutsch-als-Fremdsprache-Unterrichts, finden sich in den letzten Jahren immer mehr didaktische methodische Anregungen zu urbaner Raumwahrnehmung, urbanen Palimpsesten und Kulturraummodellen (vgl. u. a. Delhey/Jentges 2013; Cerri/Jentges 2015a; 2015b) sowie Erinnerungsorten (vgl. u. a. Schmidt/Schmidt 2007; Roche/ Röhling 2014; Badstübner-Kizik/Hille 2015; Fornoff 2016; Koreik 2018, Pasewalk/Neidlinger 2018; Fornoff/Koreik (im Druck)). Raum wird dabei als ein zentraler Aspekt zur Entschlüsselung, zum Verständnis und zur Annäherung an zielsprachige Kulturen im Fremdsprachenunterricht gesehen (vgl. Cerri/Jentges 2015b: 109). Auch sprachliche Aspekte können bei Raumerkundungen im Vordergrund stehen, vgl. hierzu u. a. Spurensuche nach Straßennamen mit Bezügen zur deutsch-polnischen Geschichte (Adamczak-Krystofowicz et al. 2012: 279; Schmidt-Bernhardt et al. 2011), Schriftspaziergänge, Beschriftung einer Stadtfassade, Suche nach eigenen Sprachspuren im Zielsprachenland (Delhey/ Jentges 2013). Die folgenden Aufgabenbeispiele (Abbildung 11 und 12) wurden 
im Rahmen des Interreg-Projekts "Nachbarsprache \& buurcultuur ${ }^{15}$ zum Thema Stadt- und Schulerkundungen (Immers 2017) entwickelt und stehen auf der Projekthomepage (http://www.ru.nl/nachbarsprache/) frei verfügbar zum Download als Word- und PDF-Dokument bereit.

Als eine besondere Form solcher Erkundungen bzw. Dokumentation des urbanen Raums können Linguistic landscape-Ansätze (vgl. u.a. Landry/ Bourhis 1997; Gorter 2006; Androutsopoulos 2008; Pütz/Mundt 2018) gesehen werden, die sich insbesondere für den Fremdsprachenunterricht anbieten, da hierbei sprachliche Zeichen - nicht nur wie im Aufgabenbeispiel in Abbildung 11 gezeigt eigen- und zielsprachige, sondern alle zu findenden Sprachspuren - im Vordergrund stehen (vgl. Cenoz/Gorter 2008).

Der Ansatz wird bisher im deutschsprachigen Raum vor allem als eine Möglichkeit des forschungsnahen Lernens an Universitäten eingesetzt (vgl. u.a. Dresing et al. 2017; Purschke 2017). Es geht um die Auswertung vorgefundener schriftlicher Sprache und Zeichen im öffentlichen Raum und somit um die (visuelle) Darstellung der sprachlichen Situation einer gegebenen Raumeinheit, z. B. einer Stadt, eines Landstrichs, einer Straße etc. (vgl. Jentges/ Sars 2018: 30). Linguistic landscaping ${ }^{16}$ kann somit eine Erweiterung der Perspektive bzw. eine neue Perspektive für die Betrachtung von bekanntem und unbekanntem Raum ermöglichen. Nachdem dieser Ansatz im letzten Jahrzehnt in der soziolinguistisch geprägten Mehrsprachigkeitsforschung an Bedeutung gewann, finden sich aktuell auch erste Publikationen für praktische Anwendung im DaF-Unterricht (vgl. die Sammelbände von Marten/Saagpakk 2017 und Badstübner-Kizik/Janíková 2018 sowie der geplante Band von Ziegler/ Marten, in denen auch unterrichtspraktische Projekte und Anregungen für die Deutsch (als Fremdsprache)-Didaktik gegeben werden).

15 Dieses Projekt wird im Rahmen des INTERREG-Programms von der Europäischen Union und den INTERREG-Partnern, dem Bundesland Nordrhein-Westfalen und der Provinz Gelderland, sowie der Taalunie finanziell unterstützt. Die Projektleitung liegt bei der Radboud Universität in Nimwegen, in Kooperation mit der Universität Duisburg-Essen, am Projekt sind derzeit (Stand Herbst 2019) gut 50 Schulen in der deutsch-niederländischen Grenzregion beteiligt. Für weitere Informationen zum Projekt siehe: http://www.ru.nl/nachbarsprache/ sowie für eine ausführliche Darstellung Sars et al. 2018 sowie Boonen et al. 2018.

16 Wir benutzen im Rahmen des Einsatzes dieses Ansatzes für den Fremdsprachenunterricht die present continuous-/progressive-Form: »linguistic landscaping «, um Nachdruck auf den sich im Verlauf befindlichen Charakter solcher Unterrichtsprojekte zu legen, bei denen keine Ergebnisse zu einer bestimmten »linguistic landscape«, sondern der Prozesse, die zu einer Annäherung an einen Ausschnitt einer »linguistic landscape« führen, offen gelegt werden. 


\section{Sprachspurensuche: auf der Suche nach Sprache!}

Die Niederlande und Deutschland sind zwei Länder, die sehr eng miteinander verbunden sind. Die deutsche und niederländische Sprache sind sich ziemlich ähnlich und deshalb ist es auch gar nicht ungewöhnlich, dass diese Sprachen ihre Spuren hinterlassen. An dich die Aufgabe, diese Sprachspuren in deiner Stadt zu finden!

1. Was sind deiner Meinung nach Sprachspuren?

2. Denkst du, dass du viele Sprachspuren in dieser Stadt findest? Und wieso denkst du das?

Aufgabe: Geht zusammen mit eurer Gruppe in die Stadt und sucht Sprachspuren! Fotografiert alles, was euch begegnet und sorgt dafür, dass ihr innerhalb der verabredeten Zeit wieder zurück seid! Nehmt eure Smartphones in die Hand und geht los!

Tipp: Sprachspuren kann man überall begegnen, denke zum Beispiel an Speisekarten, Werbungstexte oder Geschäftsnamen!

\section{Wenn ihr wieder da seid:}

3. Sammelt eure gefundenen Sprachspuren und versucht daraus eine Übersicht zu machen (z.B. zu kategorisieren oder eine Sprachlandkarte anzufertigen)

4. Was fällt auf?

5. Und haben sich deine Erwartungen bestätigt?

Abbildung 11: Erkundung des eigenen Raums: Sprachspurensuche (aus: Immers 2017: 13) 


\begin{tabular}{|c|c|}
\hline \multicolumn{2}{|l|}{ Fotochallenge: Niederlande-Deutschland Memory! } \\
\hline \multicolumn{2}{|c|}{$\begin{array}{l}\text { Zeit für ein Challenge! Geh zusammen mit deiner Gruppe in die Stadt und fotografiere, was auf diesem } \\
\text { Arbeitsblatt steht! }\end{array}$} \\
\hline \multicolumn{2}{|c|}{ Gruppenmitglieder: } \\
\hline \multicolumn{2}{|l|}{ Seid kreativ!! } \\
\hline \multicolumn{2}{|l|}{ Die Foto-Aufgaben: } \\
\hline Opdracht: & Voltooid: \\
\hline 1. Fotografiere einen Supermarkt & $\square$ \\
\hline 2. Fotografiere eine Bäckerei & $\square$ \\
\hline 3. Fotografiere einen Fahrradweg & $\square$ \\
\hline 4. Fotografiere ein Verkehrsschild & $\square$ \\
\hline $\begin{array}{l}\text { 5. Fotografiere einen Bus und/oder Bushaltestelle oder } \\
\text { Bahnhof }\end{array}$ & $\square$ \\
\hline $\begin{array}{l}\text { 6. Fotografiere einen Teil eines Wohnviertels oder einen } \\
\text { Häuserblock/ein Haus }\end{array}$ & $\square$ \\
\hline 7. Fotografiere einen Briefkasten & $\square$ \\
\hline 8. Fotografiere ein Restaurant & $\square$ \\
\hline 9. Fotografiere ein Hochhaus & $\square$ \\
\hline 10. Fotografiere das Gemeindehaus und/oder eine Kirche & $\square$ \\
\hline 11. Fotografiere eine Reihe parkender Autos & $\square$ \\
\hline 12. Fotografiere eine Ampel & $\square$ \\
\hline 13. Fotografiere etwas nach eigener Wahl & $\square$ \\
\hline
\end{tabular}

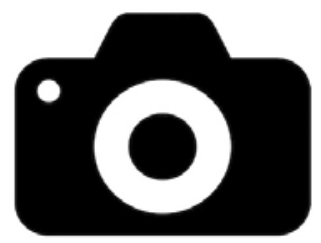

23

Abbildung 12: Erkundung des eigenen Raums: Fotochallenge Memory (aus: Immers 2017: 23) 
Der Reiz dieses Ansatzes [...] liegt in seinem Erkundungscharakter, nämlich, die eigene und fremde Umgebung aus anderen, sozusagen aus `Sprach-Augen`, zu betrachten und kennenzulernen. [...]. [Ziel ist] Sensibilisierung für Sprache an einem inner- oder außerschulischen Lernort, visuelle Wahrnehmung von Sprache und, hiermit einhergehend, Sprach- und Kulturreflexion und somit letztlich eine Möglichkeit für bedeutungsvolle und auf [kulturreflexives] Lernen ausgerichtete Programme im (Fremd)Sprachenunterricht. (Jentges/Sars 2018: 31)

Insbesondere solche mehrperspektivischen Raumerkundungen ${ }^{17}$ können der Gefahr der Entstehung von "monoperspektivistische[n] und eindimensionale[n] Weltbilder[n] « entgegenwirken (Dickel/Glasze 2009: 3), was Lernenden den Weg $\mathrm{zu}$ einer differenzierten Wahrnehmung der eigenen und unbekannten Umgebungen eröffnen und somit kulturreflexives Lernen ermöglichen kann (vgl. Jentges/Sars 2018: 29). Als Brücke in die Praxis können auf Handlungsorientierung und Mehrperspektivismus basierte Unterrichtsmodelle zur Raumerkundung und somit zum Kulturentdecken für verschiedene Zielgruppen dienen. Entsprechende Unterrichtsansätze können entsprechend auch Lehrwerken für Landeskunde Möglichkeiten zu kulturreflexiven Aufgabenimpulsen bieten.

\subsubsection{Kontaktdidaktik}

Die im vorherigen Absatz vorgestellten Ansätze für die Erkundung der eigenen Umgebung im Fremdsprachenunterricht nutzbar zu machen, schließt sehr gut an das 2007 von Badstübner-Kizik vorgestellte Konzept der Kontaktdidaktik an. Hierbei geht es darum, bestehende Kontakte, Verbindungen, bewusste oder unbewusste Bezüge von Lernenden für das Fremdsprachenlernen nutzbar zu machen, sprachlich sowie landeskundlich-kulturell:

Als Kontakt ist dabei zunächst jede Art einer Begegnung zwischen den Lernenden und den (sprachlichen und landeskundlichen) Inhalten sowie Formen der ablaufenden Lernprozesse zu verstehen, Didaktik meint dann das komplexe Feld der adressatengerechten Auswahl, Anordnung, Aufbereitung, Vermittlung und Begleitung dieser Begegnungen. (Badstübner-Kizik 2010: 100)

Theoretischer Hintergrund dieses Konzeptes ist - ähnlich wie bei urban spaceAnsätzen oder der Erlebten Landeskunde - die Annahme eines konstruktivistischen Lernprozesses, der beinhaltet, dass fremdsprachliche und -kulturelle Lernprozesse dann besonders wirkungsvoll und nachhaltig verlaufen, wenn sie,

17 Mehrperspektivisch bezieht sich hierbei sowohl darauf, beispielsweise mit Vertretern der Zielkultur den eigenen und den zielsprachigen Raum zu erkunden, als auch auf solche Raumerkundungen, die diesen aus anderen als den gewohnten Perspektiven betrachten lassen, wie beispielsweise beim linguistic landscaping. 
vom Eigenen und Bekannten ausgehend und immer wieder mittels Kontrastierung "Verbindendes und Trennendes reflektieren und Neues schrittweise erarbeiten« (Badstübner-Kizik 2010: 99).

Kulturelle, historische, geographische, soziale und andere Kontakte zwischen dem eigenen Umfeld und dem bzw. den Zielsprachenland/-ländern stärker im Unterricht zu berücksichtigen, bedeutet nicht nur die Zielkultur in den Mittelpunkt zu stellen, sondern auch das eigene Umfeld der Lernenden, beispielsweise in der Form, dass die eigene Umgebung für Fremde bzw. Gäste erschlossen werden soll (In welches Restaurant gehen wir, welches Essen wählen wir, was unternehmen wir?, Wie präsentieren wir die eigene Umgebung für Gäste?, vgl. u. a. Kistermann 2008; Brade/Krull 2016). Solche Aktivitäten regen zur Reflexion auch über das eigene Lebensumfeld an und verhindern, dass Deutschlernende zu potentiellen Touristen reduziert werden (vgl. Maijala et al. 2015:27). Auch die Wahrscheinlichkeit, dass Deutschlernende in einer realen Kommunikationssituation auf Deutsch Anderen das Brandenburger Tor präsentieren, dürfte deutlich geringer sein, als dass sie über Sehenswürdigkeiten aus der eigenen Stadt auf Deutsch berichten. Die in den Abbildungen 13 und 14 präsentierten Aufgabenbeispiele ${ }^{18}$ (Immers 2017) regen Lernende dazu an, ihre eigene Umgebung für Gäste aus ganz persönlicher Perspektive zu präsentieren.

18 Auch diese Arbeitsblätter wurden im Rahmen des Projektes »Nachbarsprache \& buurcultuur« entwickelt (vgl. Fußnote 15). 


\section{Stadtführung: eine Tour durch die Stadt!}

Bald werden niederländische Schüler eure Schule besuchen. Diese Schüler sind wahrscheinlich noch nie in eurer Stadt gewesen und sind daher sehr gespannt, was eure Stadt alles zu bieten hat; an euch die Aufgabe, ihnen eure Stadt zu zeigen!

Aufgabe: macht eine Stadtführung für die niederländischen Schüler!

Macht dies indem ihr die folgenden Fragen beantwortet. Die Antworten bilden die Route für eure eigene Stadtführung!

1. Was macht diese Stadt einzigartig?

2. Welche Fakten wisst ihr über diese Stadt? (denkt an Einwohneranzahl, Gesamtfläche, Geschichte usw.)

3. Was sind die drei schönsten oder tollsten Orte in der Stadt?

4. Welches Gebäude oder welche Gebăude sind am auffälligsten in der Stadt?

5. Was ist das tollste Geschäft?

6. Wo kann man am besten etwas essen?

7. Welcher Ort ist oder welche Orte sind weniger schön?

8. Welchen Ort findet ihr selbst komisch oder seltsam?

9. Wohin geht ihr am liebsten, wenn ihr in der Stadt seid?

10. Wo kann man die besten Pommes kaufen?

11. Wo kann man das beste Eis essen?

12. Wo kann man touristische Informationen finden?

13. Wo kann man in der Stadt übernachten?

14. Gibt es eine Kirche und wenn ja, kann man diese besuchen?

15. Welche Einrichtungen gibt es? (denkt an Kino, Schwimmbad usw.)

16. Was ist die touristische Attraktion eurer Stadt?

17. Welcher Ort hat die schönste Aussicht?

18. Wo kann am besten einkaufen?

19. Auf welcher Terrasse kann man gemütlich etwas trinken?

20. Wenn man in der Stadt ist, was sollte man dann auf jeden Fall machen?

21. Habt ihr eventuell noch andere Orte, die ihr den niederländischen Schülern noch unbedingt zeigen wollt?

Macht jetzt eine Route, sodass ihr an allen Orten vorbei kommt (die Reihenfolge ist egal!), und sie euren niederländischen Gästen zeigen könnt.

Schreibt oder zeichnet hier unten eure Route: (z.B. Startpunkt - erster Halt - Endpunkt)

Vergesst während der Stadtführung nicht, an jeder Stelle auch etwas zu erzählen! Viel Erfolg und viel Spaß!

Abbildung 13: Kontaktdidaktik: Stadtführung (aus: Immers 2017: 18) 
Schulführung: eine Tour durch die Schule!

Bald werden niederländische Schüler eure Schule besuchen. Für diese Schüler ist eure Schule noch ganz unbekannt; also an euch die Aufgabe, ihnen eure Schule zu zeigen!

Aufgabe: macht eine Schulführung für die niederländischen Schüler!

Macht dies indem ihr die folgenden Fragen beantwortet. Die Antworten bilden die Route für eure eigene Schulführung!

1. Was macht diese Schule einzigartig?

2. Welche Fakten wisst ihr über eure Schule? (denkt an Stundenplan, Pausen, Schulregeln, Schüleranzahl usw.)

3. Welches Schulfach wird wo unterrichtet? Zeigt für eure Schulfächern die drei tollsten Klassenzimmer.

4. Wo ist die Sporthalle?

5. Wo kann man in der Pause am besten sitzen?

6. Wo ist das Lehrerzimmer?

7. Wer ist der tollste Lehrer/die tollste Lehrerin?

8. Wo muss man sich melden, wenn man zu spät kommt?

9. Wo ist das Büro des Direktors/der Direktorin?

10. Welches Essen aus der Schulkantine schmeckt am besten?

11. Was ist der schönste oder tollste Ort der Schule?

12. Wo ist die nächste Bushaltestelle?

13. Wo kann man sein Fahrrad abstellen?

14. Welcher Klassenraum sollte mal renoviert werden?

15. Welche Stelle auf dem Schulhof ist am schönsten?

16. Wohin muss man, wenn man aus dem Unterricht geschickt wird?

17. Wo werden die Feste oder Veranstaltungen organisiert?

18. Wie viele Ein- und Ausgänge hat die Schule?

19. Was ist die höchste Stelle der Schule? Und was die tiefste?

20. Habt ihr noch andere Stellen, die ihr den niederländischen Schülern unbedingt zeigen wollt?

Macht jetzt eine Route, sodass ihr an allen Stellen vorbei kommt (die Reihenfolge ist egal!), und zeigt sie euren niederländischen Gästen!

Schreibt oder zeichnet hier unten eure Route: (z.B. Startpunkt - erster Halt - Endpunkt)

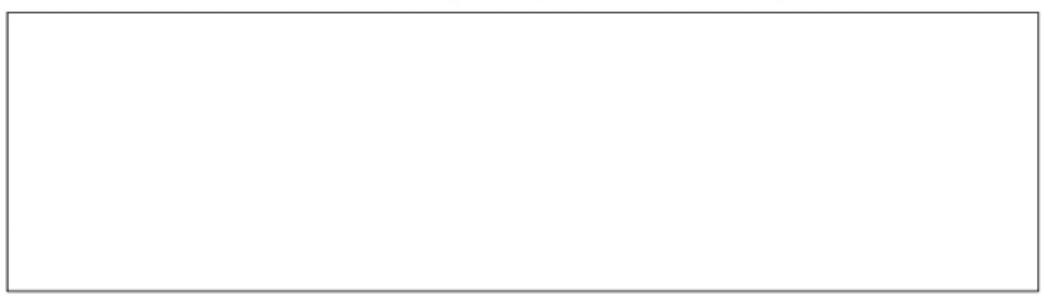

Vergesst während der Schulführung nicht, an jeder Stelle auch etwas zu erzählen!

Viel Erfolg und viel Spaß!

Abbildung 14: Kontaktdidaktik: Tour durch die Schule (aus: Immers 2017: 19) 


\subsection{Ausblick: Landes- und Kulturkunde in DaF-Lehrwerken}

Anders als für die Sprachfertigkeitsbereiche liegen keine oder eher global formulierte curriculare Vorgaben für landes- und kulturkundliches Lernen im Fremdsprachenunterricht vor, so dass Herausgeber und Lehrwerkautoren weitgehend freie Hand bezüglich der Auswahl von landeskundlichen Inhalten und deren Umfang und Position im Lehrwerk haben. Dazu kommt eine Vielzahl unterschiedlicher Positionen, insbesondere bezüglich der Ziele einer Landesoder Kulturkundedidaktik und deren theoretischer Verankerung, wie sich insbesondere bezüglich neuerer Positionen auch in ganz aktuellen einschlägigen wissenschaftlichen Beiträgen zum Thema widerspiegelt, so u.a. in dem von Schiedermair (2018) herausgegebenen Band $\mathrm{zu}$ »Deutsch als Fremd- und Zweitsprache \& Kulturwissenschaft«, hier insbesondere der Beitrag von Koreik (2018), sowie die in Druck bzw. in Vorbereitung befindlichen Beiträge von Fornoff/Koreik zum Thema »Ist der kulturwissenschaftliche und kulturdidaktische Bezug auf die Nation überholt? DACH-Landeskunde, Globalisierung und Erinnerungsorte. Eine Intervention « und deren »Bestandsaufnahme und kritische Positionierung" $\mathrm{zu}$ "Landeskunde/Kulturstudien und kulturellem Lernen im Fach DaF/DaZ«. Übereinstimmend mit einer verstärkten wissenschaftlichen Auseinandersetzung neuerer Landeskunde-Tendenzen im Fremdsprachenunterricht finden sich inzwischen einige Anregungen für die Umsetzung auch dieser neuerer theoretischer Positionen in speziellen Materialsammlungen oder in fachdidaktischen Publikationen, dennoch oder gerade deshalb gilt: Wer die Wahl hat, hat die Qual. Ernstzunehmende konkrete Handreichungen, wann und in welchem Umfang, welche landeskundlichen Themen als auch welche Didaktik der Landeskunde im Laufe eines Fremdsprachen-Lernprozesses einzusetzen wäre, existieren berechtigterweise nicht und können auch nur zielgruppen-, lernziel- und ortsabhängig getroffen werden (vgl. Absatz 3.3). Sowohl für die Konzeption von Lehrmaterialien als auch für die Gestaltung von Lehrplänen bzw. konkreten Unterrichtsentwürfen gilt: Es gibt gerade im Bereich der Landeskunde eine Vielfalt an didaktischen Herangehensweisen, Themen und Positionen, die weniger als einzelne exklusive Ansätze einander ausschließen sollten, sondern vielmehr als sich ergänzende und nicht nur additiv, sondern möglichst als miteinander verknüpft einzusetzende Möglichkeiten gesehen werden sollten, im Sinne einer ausgleichenden Landeskundepräsentation. Für die drei klassischen Ansätze der Landekunde-Didaktik gilt dies in aller Regel für heute auf dem Markt befindliche Lehrmaterialien bereits, aber neuere, v.a. kulturwissenschaftlich geprägte Ansätze sollten stärker und nicht miteinander konkurrierend Einzug in die Unterrichtspraxis erhalten, um mehrperspektivische Zugänge zu der vermeintlich eigenen und vermeintlich fremden Kultur zu erhalten: Faktisches Wissen über die Zielkulturen und kommunikative All- 
tagshandlungsfähigkeit gehört genauso hierzu wie Fähigkeiten, Strategien und Fertigkeiten im Umgang mit verschiedenen Kulturen und Gesellschaften (Handeln) und Zugang zu sowie Verständnis für deren diskursive Aushandlungsprozesse und Versuche der Bedeutungszuschreibungen (Verstehen).

In Kapitel 4 fokussieren wir in ausgewählten gegenwärtig eingesetzten Lehrwerken für Deutsch als Fremdsprache aus den Niederlanden und Polen auf die jeweils getroffenen inhaltlichen und didaktischen Entscheidungen in Bezug auf die Landes- und Kulturkunde und zeigen, welche Vielfalt an landeskundlichen Themen und Herangehensweisen angeboten werden. Die im Folgenden präsentierten Analysen geben Einblicke, wie eine konkrete Umsetzung landesund kulturkundlicher Ansätze für die Unterrichtspraxis gestaltet werden kann. Lehrende kennen in der Regel die gängigen Lehrwerke, die im eigenen Land für den DaF-Unterricht zur Verfügung stehen, ebenso die unterschiedlichen Inhalte und Methoden der Landeskundevermittlung in diesen Lehrwerken. Analysen, welche länderübergreifend die Vielfalt an landeskundlichen Inhalten und didaktischen Vorgehensweisen präsentieren, ermöglichen eine Übersicht über eine breitere landes- und kulturkundliche Landschaft und können entsprechend ein größeres Spektrum an praktisch um- und einsetzbaren Aufgaben-, Übungsund Projektanregungen zur Inspiration liefern.

Bei der Präsentation der Analysen gehen wir zuerst auf den allgemeinen Kontext des jeweiligen Lehrwerks ein. Nur ausgehend von der jeweiligen Zielgruppe und hiermit einhergehend den angestrebten Lernzielen und angenommenen/vorausgesetzten Rahmenbedingungen kann eine Analyse der landeskundlichen Inhalte, ihrer Stellung im Lehrwerk sowie der Art der Landeskundevermittlung - entsprechend aller in diesem Kapitel genannter Kriterien - in angemessener Form erfolgen. Wir beginnen mit einer globalen Beschreibung dieser Inhalte und deren Position im Lehrwerk (beispielsweise implizit vs. explizit) und gehen gesondert auf folgende Aspekte ein:

- Layout/Design

- Inwiefern wird ein Bezug zur eigenen Lebenswelt der Lernenden hergestellt, kann die Auswahl landeskundlicher Inhalte als zielgruppengerecht bezeichnet werden (vgl. Kapitel 2.2 sowie Kapitel 2.3), erfolgt die Präsentation der Inhalte und die Art der Aufgabenstellungen ausgewogen und abwechslungsreich, werden unterschiedliche Aspekte des landeskundlichen Lernens (im Sinne einer ausgleichenden Landeskunde) berücksichtigt? Dies beinhaltet auch die Frage, ob die im Lehrwerk präsentierten Inhalte für die jeweilige Zielgruppe zur Reflexion anregen, Diskurse integrieren und Diskussionen ermöglichen.

- Inwiefern wird auf verschiedenen Ebenen ein vergleichender Ansatz präsentiert? Werden im Sinne einer Kontaktdidaktik Bezüge zum eigenen Lebensumfeld der Lernenden hergestellt? Wird die Heterogenität der Sprach- 
und Kulturlandschaft (plurilinguale/-kulturelle Aspekte) der Zielsprache/kultur berücksichtigt?

- Werden landeskundliche und kulturelle Inhalte additiv und isoliert oder integrativ präsentiert? Integrativ dann im Sinne, dass eine Sprache zu lernen, immer auch bedeutet sich mit den kulturell geprägten Konventionen des Sprachgebrauchs vertraut zu machen und entsprechend eine Verknüpfung von sprachlichem und kulturellem Lernen stattfindet. Darüber hinaus stellt sich auch die Frage, inwiefern unterschiedliche Ansätze und Zielsetzungen landeskundlichen Lernens berücksichtigt werden.

Index/Überblick verwendeter Begriffe (wie diese im Rahmen der Analysen benutzt werden):

Implizite Landeskunde: im Lehrwerk bzw. den einzelnen Lektion enthalten, ohne dass Inhalte gesondert als Landeskunde bezeichnet werden $->$ häufig integrativ

Explizite Landeskunde: Gesonderte, explizit als Landeskunde bezeichnete Teile im Lehrwerk

Integrative Landeskunde: Sprach- und Landeskundeinhalte werden integriert, miteinander verknüpft angeboten $->$ häufig implizit

Kulturreflexives Lernen: Zielsprachen- und Ausgangskultur nicht nur thematisieren und vergleichen, sondern darüber reflektieren und diskutieren.

Diskursive Landeskunde: Lernende werden mit dem »fremden « Diskurs bekannt gemacht, durchschauen ihn und können die eigene und fremde Position bzw. Perspektive im Diskurs kritisch reflektieren

Kontaktdidaktik: Einbezug des eigenen Umfelds, der Kontakte und Bezüge der Lernenden mit der/den Zielsprachenkultur/en

Ausgleichende Landeskunde: Verschiedene Landeskundeansätze werden ergänzend und miteinander verknüpft eingesetzt 
Open-Access-Publikation im Sinne der CC-Lizenz BY-NC-ND 4.0

() 2020, Vandenhoeck \& Ruprecht GmbH \& Co. KG, Göttingen

ISBN Print: 9783847111214 - ISBN E-Lib: 9783737011211 


\section{Lehrmaterial-Analysen}

\subsection{Methodik}

Vor der eigentlichen Analyse wird zunächst darauf eingegangen, vor welchem Hintergrund und unter welchen Rahmenbedingungen die jeweils daran anschließend analysierten Lehrwerke produziert wurden. Dabei wird auch kurz die Position des Schulfaches Deutsch im jeweiligen Landeskontext, den Niederlanden und Polen, erläutert sowie werden die curricularen Voraussetzungen und schulischen Rahmenbedingungen, wie beispielsweise Abschlussprüfungen skizziert.

\subsubsection{Kontext}

Unser Fokus liegt jeweils auf Lehrwerken für Jugendliche im Alter zwischen 12 und 15 Jahren. In diesem Alter besuchen Jugendliche in Polen und den Niederlanden und auch im Vergleich zu Deutschland unterschiedliche Schultypen. In Deutschland und in den Niederlanden gehen die Schüler dann bereits $\mathrm{zu}$ weiterführenden Schulen; in Polen, zumindest seit der Reform aus dem Jahr 2017, besuchen die Schüler in diesem Alter noch die Grundschule. Abbildung 15 zeigt den gymnasialen Bildungsweg in den drei Ländern und markiert für das Alter 13-14 Jahre die Jahrgänge, in denen die Schüler mit dem Deutschunterricht (in Polen meistens erst als 2. Fremdsprache) anfangen. Für Polen sind das die 7. und 8. Klasse der Grundschule und für die Niederlande die 2. und 3. Klasse des niederländischen Gymnasiums, was im deutschen Schulsystem der 8. und 9. Jahrgangsstufe entspricht. Die niederländischen Schulen können sich allerdings für einen früheren Start entscheiden, nämlich in der 1. Klasse (7. Jahrgangsstufe in Deutschland). 


\section{Der gymnasiale Bildungsweg}

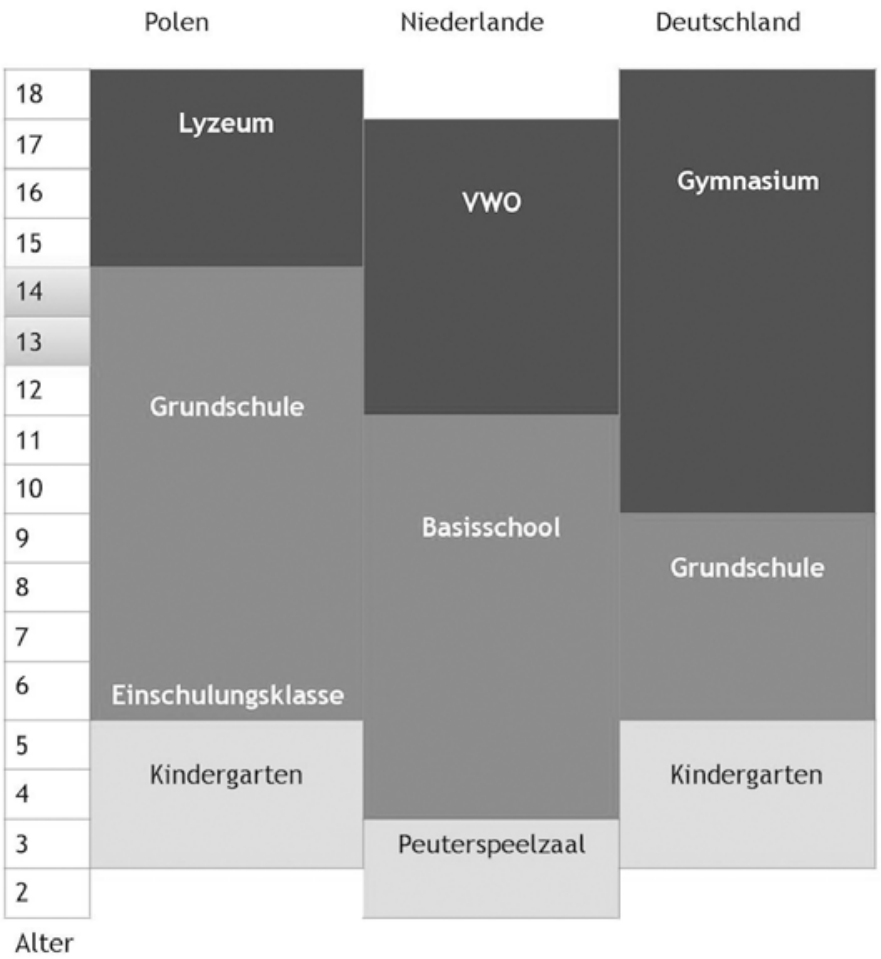

Abbildung 15: Der gymnasiale Bildungsweg in Polen, den Niederlanden und Deutschland ${ }^{19}$

\subsubsection{Analyseverfahren}

Für die Analyse wurden für beide Länder drei Lehrwerke ausgewertet, d.h. drei Lehrwerke aus den Niederlanden und drei aus Polen. Es handelt sich um die folgenden polnischen und niederländischen DaF-Lehrwerke: Mit links!, Kompass Team, Meine Deutschtour bzw. Neue Kontakte, Na klar! und TrabiTour. Wir haben uns für die $\mathrm{HAVO}^{20}$-Ausgabe der niederländischen Lehrwerke entschie-

19 Unter den niederländischen Begriffen in der Abbildung kann Folgendes verstanden werden:

- Peuterspeelzaal $=$ Kindertagestätte

- Basisschool = Grundschule

- VWO = voorbereidend wetenschappelijk onderwijs = weiterführender Bildungsgang, vergleichbar mit dem Gymnasium in Deutschland.

20 HAVO steht für hoger algemeen voortgezet onderwijs und bereitet die Schüler auf die höhere Berufsbildung vor, etwa vergleichbar mit der Real- oder Gesamtschule in Deutschland. 
den, da diese sich am ehesten mit den polnischen Lehrwerken, die ohne Angabe des jeweiligen Bildungsniveaus erscheinen, vergleichen lässt. In 4.2.1 werden die niederländischen weiterführenden Schultypen erläutert.

Im Rahmen der hier vorliegenden Analyse wurden die in den jeweils ersten zwei Lernjahren eingesetzten Lehrwerke betrachtet. Konkret heißt das für Polen, dass wir Band 1 (für die 7. Klasse) und Band 2 (für die 8. Klasse der Grundschule) der polnischen Lehrwerke analysieren. In den Niederlanden sind es der Band 1-2 (für die 2. Klasse, optional mit Start in der 1. Klasse, vgl. 4.1.1) und Band 3 (für die 3. Klasse); für die Niederlande handelt es sich um die Niveaustufen A1 und A2 nach GER, für Polen um die Niveaustufe A1. Analysiert wurden Texte und Aufgaben in den jeweiligen Kursbüchern.

In der Analyse haben wir uns auf die gedruckten Lehrwerke beschränkt. Das digitale Angebot wurde nicht gesichtet, weil das multimediale Angebot in den Niederlanden und Polen sehr unterschiedlich entwickelt ist und den Lehrenden und Lernenden nicht in gleichem Maße zur Verfügung steht. So liegt in den Niederlanden ein umfangreiches digitales Angebot vor, das auch im Unterricht benutzt wird und von Lehrenden und Lernenden als selbstverständlich empfunden wird, während dies in Polen im schulischen DaF-Unterricht bisher nicht der Fall ist.

Die einzelnen Lehrwerksanalysen starten jeweils mit einer Beschreibung des Lehrwerkes, in der der Aufbau des Kursbuches und die Thematik und Struktur der einzelnen Einheiten vorgestellt wird. Bei der Analyse der landeskundlichen Inhalte werden zuerst allgemeine Fragen gestellt, um ein Bild von der Position, die Landeskunde im Lehrwerk hat, zu bekommen. So wird zunächst evaluiert, welche Stellung Landeskunde im Buch überhaupt hat. Dabei geht es um die konkrete Position von landeskundlichen Themen im Lehrwerk und in einzelnen Einheiten und Lektionen und deren dortigen Stellenwert. Darüber hinaus gehen wir der Frage nach, welche Art der Landeskundevermittlung das Lehrwerk anbietet. Dabei wird angegeben, ob das Lehrwerk die landeskundlichen Inhalte als solche vermerkt, d.h. explizit oder vielmehr implizit anbietet. Auch wird festgehalten, ob die Landeskunde additiv, isoliert bzw. integrativ angeboten wird.

Landeskunde wird als integrativ bezeichnet, wenn Sprach- und Landeskundeinhalte integriert, d.h. miteinander verknüpft angeboten werden, wobei in den Aufgaben sprachliche und kulturelle Aspekte ‘gleichberechtigt ` vertreten sind. Wird Landeskunde isoliert angeboten, dann passt ein vorgestelltes landeskundliches Phänomen weder zum Thema der Einheit noch wird es integriert, d.h. mit Sprachtraining verknüpft, präsentiert. Landeskunde wird dagegen additiv angeboten, wenn ein vorgestelltes landeskundliches Phänomen zum Thema der Einheit passt, aber nicht mit sprachlichen Inhalten verbunden ist.

Außerdem wird beschrieben, wie Landeskunde sichtbar gemacht wird. Dabei wird u. a. auf die Verwendung bzw. die Auswahl von angebotenen Fotos, Grafiken 
usw. eingegangen. Abschließend wird analysiert, inwiefern sich die Konzepte und Ziele, wie sie in den in Kapitel 3 vorgestellten Landeskundeansätzen präsentiert wurden, in den einzelnen Lehrwerken widerspiegeln. Dabei gehen wir der Frage nach, inwiefern das DACH-Prinzip befolgt und dementsprechend die kulturelle und sprachliche Vielfalt des deutschen Sprachraums behandelt wird (siehe Kapitel 3.2). Auch wird festgehalten, inwiefern ein Lehrwerk einen Bezug zur Lebenswelt der Lernenden, konkret der Zielgruppe der 12-15 Jährigen, herstellt, wobei nach den Einsichten der Kontaktdidaktik (siehe 3.5.4) ermittelt wird, ob die Aufgaben die Möglichkeit bieten, die ausgangs- und zielsprachige Umgebung zu entdecken und das Herstellen von Verbindungen zwischen beiden ermöglichen. Entsprechend wird eingestuft, inwiefern ein Lehrwerk die landeskundlichen Inhalte zielgruppengerecht, d.h. nach dem Prinzip der Zielgruppenorientierung (siehe 3.3) anbietet. Außerdem wird herausgearbeitet, ob ein Lehrwerk eine kulturreflexive Haltung fördert, wobei die vermeintlich eigene und fremde Kultur verglichen werden und eine kritische Haltung gegenüber der eigenen (Anfangs)perspektive initiiert wird (siehe 3.5.1). Auch wird bestimmt, ob ein Lehrwerk Aktivitäten anbietet, die den Anforderungen der diskursiven Landeskunde entsprechen und es den Lernenden ermöglichen, am zielsprachigen Diskurs teilzunehmen (siehe 3.5.2). Des Weiteren versuchen wir, zu ermitteln, inwiefern ein Lehrwerk die Lernenden Landeskunde und deren Inhalte nach den Prinzipien der Erlebten Landeskunde (siehe 3.4.2) direkt und affektiv erfahren lässt und mit Wahrnehmungen verbindet (siehe 3.5), also beispielsweise, ob ein Lehrwerk Aufgaben enthält, die die Schüler kulturelle oder sprachliche Spuren suchen lassen. Dabei kann die sogenannte Spurensuche sowohl im Zielsprachenland als auch an außerschulischen Lernorten in der eigenen Umgebung der Lernenden mit authentischen Objekten oder Personen aus dem Zielsprachenland stattfinden.

\subsection{Landeskunde in niederländischen Lehrwerken}

Aus deutscher Sicht fallen zwei Aspekte im niederländischen Schulsystem auf. Erstens ist das frühe schulische Eintrittsalter von niederländischen Kindern festzuhalten. Eingeschult werden die meisten Kinder mit vier Jahren, wobei anzumerken ist, dass die ersten zwei sogenannten Schuljahre eher KindergartenCharakter haben. Schulpflichtig sind die Kinder allerdings erst mit fünf Jahren. Zweitens wird in den Niederlanden länger gemeinsam gelernt; der Übergang zur weiterführenden Schule erfolgt im Alter von ca. 13 Jahren. Die nach deutschem System in aller Regel als Jahrgangsstufen 5 und 6 bezeichneten ersten beiden Schuljahre an weiterführenden Schulen werden in den Niederlanden noch an der Grundschule verbracht. Die drei weiterführenden Schultypen in den Nieder- 
landen sind: VMBO (Voorbereidend Middelbaar Beroepsonderwijs), in etwa mit der deutschen Hauptschule vergleichbar, HAVO (Hoger Algemeen Voortgezet Onderwijs), vom Niveau her eher etwas über der deutschen Realschule anzusiedeln, und VWO (Voorbereidend Wetenschappelijk Onderwijs) entspricht dem deutschen Gymnasium. Verlage entwickeln einzelne Ausgaben eines Lehrwerkes für jeden dieser Bildungswege. Für das erste Lernjahr Deutsch bieten einige Verlage ein Buch, das sowohl für HAVO als auch VWO zu benutzen sei, an; ab dem zweiten Lernjahr differenzieren alle Verlage nach HAVO und VWO und entwickeln verschiedene Bände für Lernende an den beiden Schultypen.

Ein formales, präskriptives Curriculum gibt es in den Niederlanden nicht. Es gibt nur globale Abschlussqualifikationen, die für die jeweiligen Schulfächer aufgestellt wurden (CVE 2012: 24f.), wobei für die Fremdsprachen sechs Lernbereiche unterschieden werden (Sprechen, Hören, Lesen, Schreiben, Literatur, Orientierung auf Studium und Beruf). Seit 2007 sind die Sprachfertigkeitsziele an den GER gekoppelt. Für Landeskunde, Kulturvermittlung oder Interkulturalität bzw. Kulturreflexivität sind in diesem Dokument keine Abschlussqualifikationen formuliert und sie werden auch nicht in den anderen Qualifikationsbereichen erwähnt (vgl. Maijala et al. 2016; Tammenga-Helmantel et al. 2017). Die zentrale Abschlussprüfung für Deutsch, wie auch für andere moderne Fremdsprachen besteht ausschließlich aus Leseverstehen und macht $50 \%$ der Abschlussnote aus; die anderen $50 \%$ werden schulintern geprüft und können aus (Teilen) der genannten sechs Prüfungsbereichen bestehen.

In aller Regel kann Deutsch nach Englisch und neben Französisch als Pflichtfremdsprache in den Niederlanden gesehen werden, wobei niederländische weiterführende Schulen selber entscheiden können, ab wann sie Deutsch anbieten. Üblicherweise wird Deutsch ab der zweiten Klasse (Alter: 13-14 Jahre) unterrichtet, aber Schulen können Deutsch auch bereits in der 1. Klasse anbieten. Nach dem deutschen Schulsystem wären das die 7. bzw. 8. Jahrgangsstufe. Verlage bieten in der Regel ein Anfängerbuch mit der Bezeichnung »1-2« an, das für die ersten beiden Klassen der weiterführenden Schulen gedacht ist (siehe auch unten). Für HAVO und VWO-Schüler gilt in aller Regel, dass sie mindestens zwei Jahre Deutsch haben und es dann als Prüfungsfach in der Sekundarstufe II wählen können. Auch VMBO-Schüler können sich bei der Abschlussprüfung für das Wahlfach Deutsch entscheiden. Der Trend für das Schulfach Deutsch ist steigend mit 411.895 Deutschlernenden, davon $380.000 \mathrm{im}$ Schulbereich (Stand 2015; Datenerhebung Goethe-Institut).

In den Niederlanden gibt es für Lehrwerke keine Zulassungsverfahren. Verlage und Autorenteams haben in der Zusammenstellung der Lehrwerke freie Hand und orientieren sich dabei stark an den Wünschen der Lehrer, ihren Kunden. Die Autorenteams der DaF-Lehrwerke bestehen oft aus Deutschleh- 
rern; manchmal ist auch ein Fachdidaktiker aus der Lehrpersonenausbildung an Hochschulen oder Universitäten an der Arbeit beteiligt. So ungefähr alle vier Jahre erscheint eine neue Ausgabe eines Lehrwerkes. Für das Schulfach Deutsch gibt es momentan fünf niederländische Lehrwerke: Neue Kontakte, Na klar!, TrabiTour, Salzgitter heute, Zugspitze wobei letzteres Salzgitter heute ablöst. Dazu kommen zwei regionalisierte globale Lehrwerke, die für den niederländischen Markt adaptiert wurden: Team Deutsch und Fantastisch! DeutschFachgruppen an Schulen entscheiden sich in aller Regel für die Arbeit mit einem dieser Lehrwerke. Der Einsatz von auf dem deutschsprachigen Markt produzierten DaF-Lehrwerken erfolgt in der Praxis nur sehr vereinzelt. In der vorliegenden Analyse werden die drei Lehrwerke mit dem größten Marktanteil berücksichtigt: Neue Kontakte, Na klar! und TrabiTour (vgl. Hoch et al. 2016).

Eine - zumindest aus deutscher oder polnischer Sicht - Besonderheit bei niederländischen Lehrwerken sei noch vorab erwähnt: Ein niederländisches Lehrwerk besteht in der Regel aus einem Text- und einem Aufgabenbuch, die beide im Unterricht benutzt werden. Das Textbuch bietet Texte, Wörterlisten und eine Grammatikübersicht, aber keine Aufgaben. Aufgaben finden sich im Arbeitsbuch, das somit nicht als optionaler Teil angesehen werden kann, sondern in vielen Fällen als Ausgangspunkt gilt. Das heißt konkret, dass die niederländischen Schüler mit dem Arbeitsbuch beschäftigt sind und darin Verweise auf Texte und Grammatik im Textbuch finden. Ein Textbuch hat dann eher die Funktion eines Nachschlagewerks, welches in Polen und Deutschland dagegen Lesetexte, Wortschatz- und Grammatikübersichten und Aufgaben anbietet.

\subsubsection{Neue Kontakte}

Das Lehrwerk Neue Kontakte bietet für die ersten zwei Jahre Deutsch zwei Bände an. Für das erste Lernjahr wird Neue Kontakte 1-2 t/havo/vwo (2013; GER A1A2) eingesetzt. Es besteht aus einem Textbuch mit sechs Einheiten. $\mathrm{Zu}$ den Einheiten 1-3 gibt es Arbeitsbuch 1; Arbeitsbuch 2 ergänzt die Einheiten 4-6. Mit einem Aktivierungscode haben die Lernenden Zugang zu Online-Materialien (Extra-Aufgaben, Hörfragmente und Links). Die Einheiten sind thematisch wie folgt betitelt: Sprich mit!; Freizeit!; Schmeckt's?; Im Kaufrausch; Wie wohnst du? und Unterwegs. Das Textbuch bietet Texte zu den Aufgaben im Arbeitsbuch, Lerntipps, ein kontrastives Wörterverzeichnis, eine Grammatikübersicht und eine Landkarte von Deutschland, Österreich und der Schweiz an. Liechtenstein steht ebenfalls auf der Karte, wird aber in der Überschrift der Karte nicht genannt. Neue Kontakte 3 havo/vwo (2014; GER A2-B1) - für das zweite Lernjahr Deutsch - hat einen vergleichbaren Aufbau wie der erste Band; die Struktur dieses Bandes ist mit der im Band 1-2 identisch. Die Thematik der 
einzelnen Einheiten ist: Hilfe! (Dienstleistung); An die Arbeit (Studium und Beruf); Abschalten (Freizeit); Gesund und glücklich (Körper); Lebenswelten (Politik und Gesellschaft) und Fernweh (Reisen).

Das Arbeitsbuch ist leitend; die Lernenden benutzen es als Ausgangspunkt und werden regelmäßig dazu aufgefordert, einen Text oder Grammatik im Textbuch nachzuschlagen.

Im Arbeitsbuch wird zu jeder Aufgabe angegeben, welcher Kategorie die Aufgabe angehört. Dabei werden folgende Kategorien unterschieden: Sehen, Lesen, Grammatik, Sprechen, Wörterbuch, In der Klasse, Landeskunde, Hören, Sprechen, Schreiben, Praxis, Extra Hören und Extra Lesen. Neue Kontakte 3 bietet zusätzlich noch Sprache und Jugendliteratur. Die Aufgaben werden pro Kategorie angeboten. Das heißt zum Beispiel, dass alle Schreibübungen einer Einheit zusammen als Block präsentiert werden. Zur Hälfte und am Ende einer Einheit wird den Lernenden die Möglichkeit geboten, einen diagnostischen Test zu machen. In der sogenannten Nachbereitung können die Lernenden prüfen, ob sie die Lernziele der Einheit erreicht haben. Die Lernziele beziehen sich alle auf den Spracherwerb, und das GER-Niveau wird dabei angegeben, z. B. "A1 Du lernst die langen Vokale richtig auszusprechen« (NeKo 1-2, Einheit 5). Jede Einheit schließt mit einer praxisorientierten Aufgabe mit GER-Angabe, z. B. das Erstellen eines Blogs, und mit mehreren Seiten an Wiederholungsmaterialien ab.

\subsubsection{Stellung und Art der Vermittlung von Landeskunde in Neue Kontakte}

Landeskunde scheint in Neue Kontakte eine ambivalente Position einzunehmen. Sie erscheint zwar mit z. B. Sprechen, Lesen und Grammatik in der allgemeinen Übersicht zum Lehrwerk Neue Kontakte, wird aber genauso wie auch Praxis und Extra Hören/Lesen farblich von den Fertigkeiten abgehoben. Die Lehrerhinweise geben hierzu keine weiteren Erläuterungen, allerdings scheint die farbliche Abhebung als Abgrenzung gedacht zu sein. So werden in den Lernzielen, die am Anfang einer Einheit formuliert werden, keine landeskundlichen Themen erwähnt, und ebenfalls in den im Lehrwerk angebotenen Tests wird Landeskunde nicht berücksichtigt. Auch in der Lernübersicht, die das Lehrwerk am Ende jeder Einheit bietet, finden sich keinerlei landeskundliche Inhalte. Hinzukommt, dass ein Teil der Landeskundeaufgaben mit einem $v$ (= extra Lehrstoff) markiert ist oder in >Extra-Lesen ^oder `Extra-Hören` aufgenommen worden ist. Das Signal, das hiermit für den Deutschunterricht vermittelt wird, dürfte sein: Landeskunde ist fakultativer Teil und gehört nicht zum Kern des Fremdsprachenunterrichts, der sich auf Spracherwerb richtet.

Neue Kontakte bietet Landeskunde in der Regel explizit an. So enthält jede Einheit einen Text, der explizit als Landeskunde bezeichnet wird und dazu zwei bis drei Aufgaben im Arbeitsbuch. Fast immer betrifft es einen einseitigen Text 
mit Foto, zu dem die Lernenden Fragen zum Bild und Textverständnis beantworten sollen. Die landeskundlichen Themen, die besprochen werden, schließen in der Regel an das Thema der Einheit an, beispielsweise wird in der Einheit >Wie wohnst du? ? präsentiert, wie ein deutsches Mehrfamilienhaus aussieht (NeKo 12: 79). Und im Fertigkeitsteil der Einheit findet sich noch ein Text zum Leben auf einer Alm (NeKo 1-2: 81). In der Einheit `Im Kaufrausch ‘ wird ein Text mit Foto vom CentrO Oberhausen im Landeskundeteil präsentiert (NeKo 1-2: 63). Anschließend folgt ein Lesetext zu einem Tauschladen in Kreuzberg (NeKo 1-2:65).

Für verschiedene Aufgaben, die auf das Fördern der einzelnen Fertigkeiten abzielen, gilt, dass sie implizit landeskundliche Inhalte vorstellen. Oft werden dabei kulturtragende Texte für Leseverständnistraining eingesetzt, ohne auf die kulturellen Inhalte einzugehen. Landeskunde wird mithin präsentiert, aber nicht integrativ vermittelt. So erstellen die Lernenden im Abschnitt >Praxis` ein Kochvideo und bereiten Kaiserschmarrn zu (NeKo 1-2/1: 198f.), aber darüber hinaus fehlen Hinweise auf beispielsweise die Herkunft oder die Region, aus der Kaiserschmarrn stammt. Andere Beispiele wären eine Aufgabe, in der die Lernenden zu zweit eine Wegbeschreibung üben und dabei authentische Stadtpläne von Ulm und Neubrandenburg (NeKo 1-2/2: 181f.) und den U-Bahn-Plan von Berlin (NeKo 3/B: 201) verwenden, oder während des Hörens die Modalverben im Liedtext Müssen nur wollen der Band Wir Sind Helden ergänzen sollen (NeKo 1-2/2: 147 f.). Landeskunde dient hier sozusagen zur Einübung von morphosyntaktischen Formen. Auf diese Weise arbeiten die Lernenden an ihrer sprachlichen Kompetenz, und Landeskunde beinhaltendes Material wird als Mittel dazu eingesetzt.

Auch mittels name dropping wird Landeskunde implizit angeboten, wie etwa in einer E-Mail von Thomas, in der er eine Ferienwohnung reserviert und im P.S. fragt, ob die Inhaberin doch bitte noch mal eine so tolle Sachertorte backen könnte (NeKo 3: 110). Des Weiteren werden insbesondere in den Grammatikübungen gelegentlich geographische Namen genannt, wie etwa Rügen, Leipzig, Salzburg, Hannover und Stuttgart. Auch mittels Logos vermittelt Neue Kontakte implizit landeskundliche Information, beispielsweise: Deutsche Post (NeKo 3: 20), Deutsche Bahn und ADAC (NeKo 3:21).

Das Lehrwerk bietet Landeskunde oft in Form eines Textes, der mit Farbfotos oder einer Zeichnung illustriert wird, an. Sie bilden den Gegenstand des Textes ab, wie zum Beispiel das Kopfüber-Haus auf Usedom (NeKo 1-2: 84) oder verschiedene Brötchensorten in einem Text zu der großen Brotvielfalt in Deutschland. Neben Fotos werden gelegentlich auch Grafiken eingesetzt, z. B. um zu zeigen, wo man Deutsch spricht (NeKo 1-2:15). Für die Texte, die nicht unter Landeskunde fallen, gilt, dass sie fast immer auch visuell unterstützt werden, meist mit Bildern von Personen oder Gegenständen, jedoch ohne zielsprachenkulturelle landeskundliche Inhalte zu präsentieren. 


\subsubsection{Landeskundeansätze und Prinzipien in Neue Kontakte}

Vielfalt der deutschen Kultur- und Sprachlandschaft

Das Lehrwerk zeigt die Vielfalt des deutschen Kulturraums, indem es Traditionen, Essgewohnheiten, Feiertage und Gebiete aus den DACH-Ländern in Aufgaben, Bildern und Texten vorstellt. Von den DACH-Ländern steht Deutschland in Neue Kontakte deutlich an erster Stelle; Österreich und der Schweiz wird in etwa gleich wenig Aufmerksamkeit gewidmet. Inhalte zu diesen beiden Ländern beschränken sich in der Regel auf touristische Sehenswürdigkeiten, d.h. Alpenlandschaften und regionale Speisen (siehe auch Maijala et al. 2016).

Positiv fällt der plurilinguale Ansatz in Neue Kontakte auf. Die Vielfalt der deutschen Sprache wird ab Neue Kontakte 3 thematisiert. Jede Einheit enthält einen Abschnitt zur Sprache, in dem in einem kurzen Sachtext z. B. ein Dialekt oder eine Standardvariante mit einigen Besonderheiten und Beispielen vorgestellt wird, wie Plattdeutsch, Schwyzerdütsch, Österreichisch, Berlinerisch und deutsche Lehnwörter in anderen Sprachen. Die Aufgaben dazu fokussieren auf die rezeptiven Fertigkeiten. Die Lernenden lesen zunächst einige Wörter; anschließend hören sie ein Fragment, in der Regel einen Liedtext einer regionalen Band, und übersetzen zum Beispiel Wörter aus einem schwyzerdütschen Song, Mani Matter von Dr Alpeflug, dann beantworten sie dazu Textverständnisfragen (NeKo 3/A: 175ff, vgl. Abbildung 16a und b) oder sie hören ein Lied in österreichischem Dialekt, beispielsweise Liedermacher von 2erBeziehung (NeKo 3/B: 26). Das Verständnis des jeweiligen Dialektes wird auch explizit in den Lernzielen, die am Anfang der Einheit formuliert werden, genannt. 
c Vul in elke zin de passende vertaling van 'blad' in.
10 Hast du ein anderes
Papier für mich?
11 Schau mal, auf dem sind viele Läuse!
12 Mein Vater, der gerne bastelt, hat für den Tisch eine Eichenholz gemacht.
13 Stelle bitte die Tassen auf das ich bringe sie dann in die Küche.

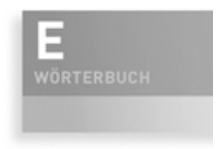

\title{
26 Schwyzerdütsch
}

\section{a Beantworte die Frage.}

1 Ben je wel eens in Zwitserland geweest? Welke taal/talen heb je daar gehoord?

b Lies Text F im TB auf Seite 48.

c Du hörst die Wortpaare aus dem TB. Sprich sie in den Pausen nach.

\section{Schwyzerdütsch II}

a Du hörst einige Sätze auf Schwyzerdütsch, dem Schweizer Dialekt des Deutschen. Kreuze an, was die Sätze auf Hochdeutsch bedeuten.

1

A Der Test ist einfach!

B Der Text ist einfach crazy!

A Stefan, geht es dir gut?

B Stefan, sei so gut und mach das Fenster zu.

3

A Ich habe das Buch, von dem er mir erzählt hat.

B Ich habe ein Buch, das er mir gegeben hat.

4

A Einen trinken gehen.

D E Eis essen gehen.

5

A Zeit ist am wertvollsten, wo immer man sie gerade hat.

B Zeit ist am wertvollsten, wenn man sie nicht hat.

\author{
b Du hörst die Sätze noch mal. Versuche sie in den Pausen \\ nachzusprechen. \\ 1 Dä tescht isch eifach gsi! \\ 2 Stefan, gang bis so guet s Faischter go zuemache. \\ 3 I han es Buech, won ăr mir ggäă het. \\ 4 Eis go zieh. \\ 5 Ziit isch am wertvollschtă, wämmer si net hăt.
}

Abbildung 16a: Schwyzerdütsch (Neue Kontakte 3/A: 175) 


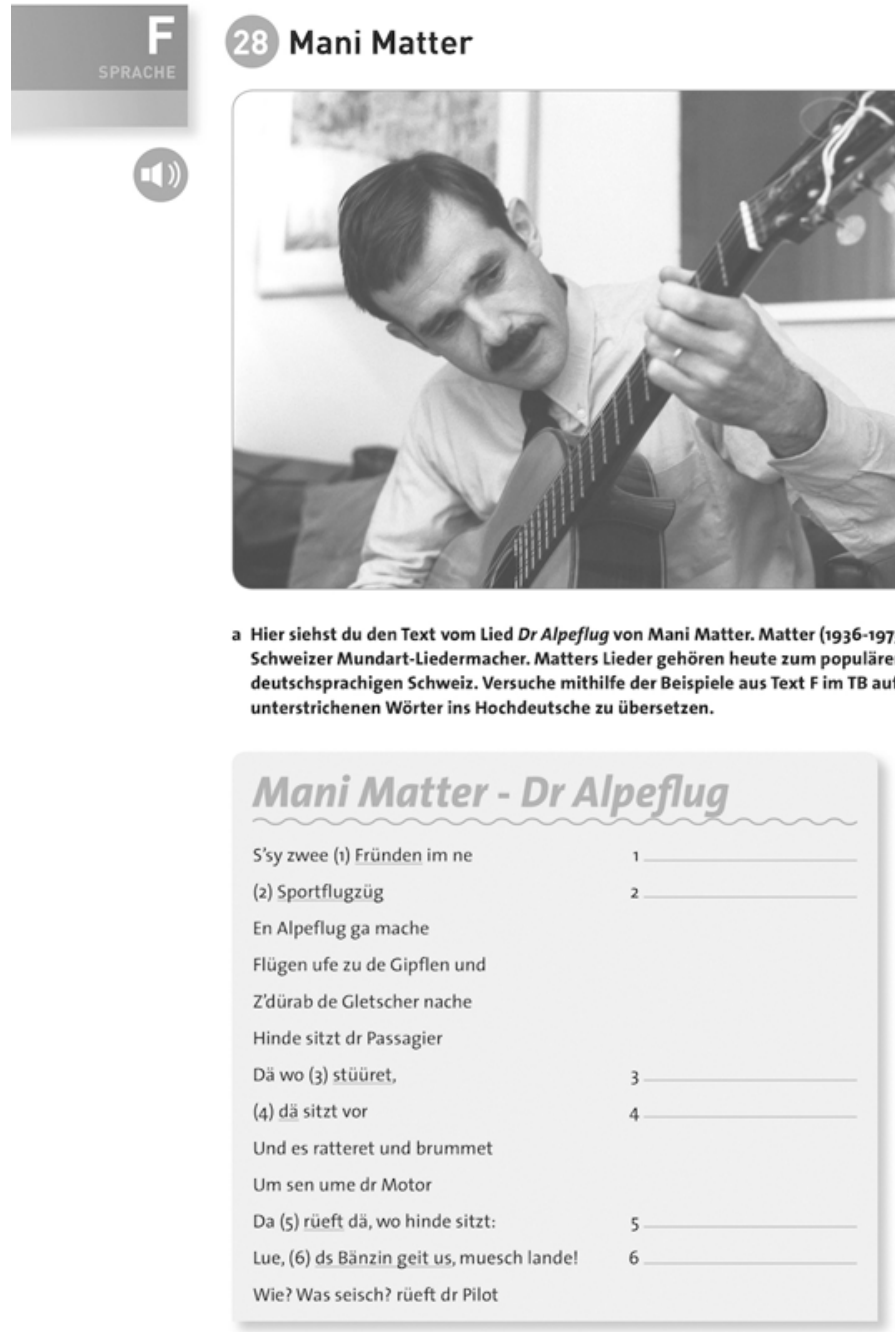

Abbildung 16b: Schwyzerdütsch (Neue Kontakte 3/A: 176) 


\section{Bezug zur Lebenswelt der Zielgruppe}

Offensichtlich ist, dass versucht wurde, das im Lehrwerk präsentierte Material auch inhaltlich mit der Lebenswelt von Jugendlichen zu verbinden. Erstens bietet Neue Kontakte Alltagskultur an. Auffällig ist dabei die relativ große Anzahl von touristischen Attraktionen (CentrO, Kopfüber-Haus, U-Bahn-Cabrio-Tour in Berlin und Alpeniglu in Tirol), bei denen es sich für die Zielgruppe um potentiell interessante touristische Sehenswürdigkeiten handeln dürfte, d.h. solche Attraktionen, die (auch) junge Besucher anziehen. Es geht dabei nicht um die großen Highlights auf der UNESCO-Weltkulturerbe-Liste, sondern vor allem erheiternde Besonderheiten, die man im deutschsprachigen Raum findet und die zum Thema der Einheit passen. Zweitens wird bei der Beantwortung von Textverständnisfragen $\mathrm{zu}$ Texten mit landeskundlichen Inhalten fast immer versucht, den präsentierten zielsprachigen Inhalt an die persönliche Lebenswelt der Lernenden anzuschließen. Als Beispiel hierfür kann ein Text dienen, in dem deutsche Haustypen besprochen werden (NeKo 1-2/2: 91f.) und diese anschließend mit der eigenen Wohnsituation verglichen werden, indem die Lernenden gefragt werden, in welchem Haustyp sie gerne leben würden und wie sie den Keller, falls vorhanden, nutzen würden. Themen, die in Text- und Arbeitsbuch als Landeskunde vorgestellt werden und bei denen auch die persönliche Situation der Lernenden mit einbezogen wird, sind z. B.: deutsche Schultypen (Hauptschule, Realschule, Gymnasium), die Schulkantine, AGs in der Schule, deutsches Brot, Berlinale, die Jugendfeuerwehr, CentrO Oberhausen und Freitag-Taschen. Drittens werden die Lernenden manchmal eingeladen, aktiv zu recherchieren und dabei moderne Kommunikationsmittel und Internetsuchmaschinen, die in der Regel in den Alltag der Jugendlichen fest integriert sind, einzusetzen, wie zum Beispiel in den Aufgaben zu einem Lesetext über den deutschen Spiele-Autor Reiner Knizia. Die Lernenden sollen im Internet recherchieren, wie das Spiel Keltis in den Niederlanden heißt und ob es auch in den Niederlanden für Spielepreise nominiert wurde (NeKo 3/A: 107).

\section{Stimulierung einer kulturreflexiven Haltung}

Vereinzelt gibt es Aufgaben, in denen Lernende die vermeintlich eigene und fremde Kultur vergleichen sollen, beispielsweise nach dem Lesen einer Broschüre und dem Betrachten eines Videos über die deutsche und niederländische Jugendfeuerwehr (NeKo 3/A: 26f.), woran anschließend beide Jugendfeuerwehren verglichen werden sollen. Ein expliziter Vergleich mit ausdrücklich eingefordertem Urteil der Lernenden dazu wird allerdings nur zum Thema Mülltrennung angeregt (NeKo 3/B: 104f.). Hier sollen die Lernenden nach Lektüre eines Textes in Form eines Posters angeben, welche Unterschiede es bei der Mülltrennung in Deutschland und am eigenen Wohnort gibt. Abgeschlossen wird diese Aufgabensequenz mit der Frage, wo die Mülltrennung nach Ansicht 
der Lernenden besser organisiert ist. Diese multiperspektivische Aufgabe mit authentischem Text kann eine kulturreflexive Haltung fördern. Meist findet allerdings kein so ausführlicher Vergleich statt, sondern sollen die Lernenden sich lediglich überlegen, wie es wäre, wenn es x oder y in den Niederlanden gäbe. So wird z. B. zu einem Text über Wölfe in Deutschland gefragt, welche Probleme die Lernenden erwarten, sollten Wölfe in Zukunft auch in den Niederlanden auftauchen (NeKo 1-2/2: 164f.).

Ein gelungenes Beispiel, in dem ein sprachlicher Vergleich gemacht wird, zeigt eine Aufgabe, bei der Berlinerisch in einem Lesetext vorgestellt und der Zusammenhang zwischen dieser regionalen Variante und dem Niederländischen befragt wird, vgl. Abbildung 17.

In einer weiteren Aufgabe werden einige berlinerische Ausdrücke dem Hochdeutschen gegenübergestellt. Abschließend wird gefragt, welche Wörter fast identisch mit dem Niederländischen sind (NeKo 3/B: 101). Aufgaben wie diese dürften eine sprachreflexive Haltung fördern und zum plurilingualen Sprachbewusstsein der Lernenden beitragen.

\section{Landeskunde erfahren}

Neue Kontakte bietet keine Aufgaben oder Projekte, die vor Ort, z. B. im Rahmen eines Besuches im Zielsprachenland oder in der eigenen Umgebung, die deutsche Kultur oder Sprache direkt erfahren lassen. Die Lernenden sollen zwar selber ein Sprachdorf im Klassenzimmer einrichten, aber nicht deutsche Muttersprachler, die es in den Niederlanden genügend gibt, sondern sie selbst übernehmen - verkleidet - beispielsweise die Rolle eines Polizisten oder eines ADAC-Mitarbeiters (NeKo 3/A: 53f.).

\section{Fazit}

Bei den in diesem Lehrwerk angebotenen Landeskundeaufgaben ist das Aufgabenschema fast immer gleich: Sachkenntnisse werden in einem Lesetext präsentiert, anschließend abgefragt und abschließend personalisiert, indem der landeskundliche Inhalt mit der Lebenswelt der Lernenden in Bezug zu setzen versucht wird. Der Ansatz ist damit überwiegend faktenorientiert. Ein kritischer Ansatz zur kulturellen Reflexion von vermeintlich fremder und eigener Kultur bleibt weitestgehend aus. Die Niederlande selbst sind kein Thema in diesem Lehrwerk. Die Lernenden müssen zwar des Öfteren ein DACH-Phänomen mit der persönlichen Lage oder mit der Situation in den Niederlanden vergleichen, dies wird aber nicht in konkreten Anwendungsaufgaben umgesetzt, wie beispielsweise der Präsentation eines niederländischen Phänomens oder der eigenen Umgebung in der Zielsprache Deutsch; entsprechend finden sich auch keine Bilder oder Infotexte zu den Niederlanden im Lehrwerk. 


\section{So quatscht Berlin}

Lies Text F im TB auf Seite 84 und beantworte folgende Fragen.

1 Welke drie voorbeelden uit de tekst lijken sterk op Nederlands?

2 In welke twee lange klinkers zijn overeenkomsten tussen Nederlands en Berlinerisch te herkennen?

3 Welke verklaring wordt er gegeven voor de diverse invloeden waaruit het Berlinerisch is ontstaan?

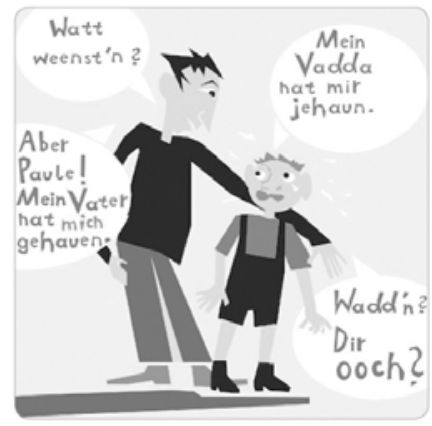

4 Bekijk de cartoon. Het Berlijns staat er ook om bekend dat dat de derde en de vierde naamval door elkaar worden gebruikt. Om welke verwisseling gaat het in deze grap en kun je uitleggen wat de grap is?

\section{Berlinerisch}

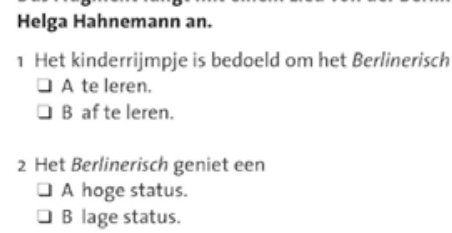

1 Het kinderrijmpje is bedoeld om het Berlinerisch

a te leren.

D a te leren.

2 Het Berlinerisch geniet een

A hoge status.

B lage status.

Abbildung 17: So quatscht Berlin (Neue Kontakte 3/B: 99) 
Inhaltlich richtet sich die Landeskunde auf die Alltagskultur und die deutsche Sprachenlandschaft, sie wird entsprechend oft additiv angeboten. Es fehlen kulturelle oder sprachliche Ausarbeitungen, Landeskunde steht vielmehr im Dienste des Sprachfertigkeitstrainings, meistens betrifft es Leseverständnisaufgaben, d.h. Textverständnisfragen zu kulturgeprägten Texten, vereinzelt wird Landeskunde in Hör- und Sprechübungen angewendet. Regelmäßig wird Landeskunde implizit angeboten, z. B. in Bildern oder mittels sogenanntem name dropping.

\subsubsection{Na klar!}

Das Lehrwerk Na klar! bietet für die ersten zwei Jahre Deutschunterricht pro Jahr einen A- und einen B-Band an, der anders als es in den Niederlanden üblich ist, Text- und Arbeitsbuch integriert. Zu jedem Band zeigt die Einleitung eine Übersicht, wo welche mittels GER-Niveaus spezifizierten Fertigkeitsziele, Grammatik und Aussprache eingeübt werden. Jeder Band enthält drei Einheiten, die als Titel den Namen einer Stadt im deutschen Sprachraum tragen und die jene Stadt mit dem Thema der Einheit verbinden. So werden in Na klar! 1-2 havo/vwo A + B (2017) für das erste Lernjahr folgende Städte und Themen präsentiert: Hamburg (sich vorstellen); Berlin (Film, Musik); Wien (Essen); Köln (Mode, Geld); München (Sport, Schule); Zürich (Reisen). Für das zweite Jahr werden in Na klar! 3 havo A + B (2018) Salzburg (Sommerferien); Stuttgart (Natur, Umwelt); Bern (Gesundheit, Ernährung); Leipzig/Dresden (Wohnumgebung); Frankfurt (Musik, Film, Bücher); Bremen (Erfindungen, Marken) präsentiert. Jede Einheit besteht aus sechs Lektionen; die Lektionen 1-3 fokussieren auf die rezeptiven Fertigkeiten; in den letzten drei Lektionen wird auf Sprachproduktion in Form von Sprechen hingearbeitet. Jede Einheit beginnt mit einer Übersicht der Lernziele - auch hier wiederum untergliedert in GERSprachfertigkeits- und Grammatikziele - und wird mit einer Übersicht der in der Einheit präsentierten Redemittel und Wortschatz abgeschlossen. Jeder Band schließt mit acht Seiten >Differenzierung` (Wiederholung; kreative Sprachübungen; Literatur) und einer >Toolbox` (eine DACH-Karte und Wortschatz) ab.

Die Lernenden arbeiten mit einem Kursbuch, das Texte, Grammatik, Wortschatz und Aufgaben enthält. Dazu gibt es noch eine digitale Lernumgebung mit Grammatik- und Wortschatztrainer, auf die die Lernenden sowohl in der Schule als auch zu Hause Zugriff haben. Mit deren Hilfe können die Lernenden adaptiv Grammatik und Wortschatz üben, abhängig davon, wie sie abschneiden, bieten die Software-Programme den Schülern passende neue Aufgaben an: entweder sollen die Schüler bestimmte grammatische Phänomene oder Vokabeln wiederholen, oder das Programm bietet neue Lerninhalte. Sie enthält ebenfalls 
Hörmaterialien, Videos und diagnostische Tests, $\mathrm{zu}$ denen die Lernenden Feedback erhalten können. Zusätzlich zu den vier Bänden bietet Na klar! ein sogenanntes Handbuch mit Grammatikübersicht, Fertigkeitstraining, Wortschatz und Beispielbriefen an. Im Kursbuch wird regelmäßig auf das Handbuch und das digitale Lernangebot verwiesen, vor allem auf den Grammatiktrainer und die Hörmaterialien mit zusätzlichen Aufgaben. Eigene Blogeinträge, Videos usw. können die Lernenden in einem digitalen Portfolio speichern.

\subsubsection{Stellung und Art der Vermittlung von Landeskunde in Na klar!}

Landeskunde nimmt eine zentrale Position im Lehrwerk ein. So tragen die Einheiten nicht nur den Namen einer Stadt aus Deutschland, Österreich oder der Schweiz und beginnen jeweils mit einer Collage jener Stadt samt folgender Orientierung auf die Stadt und auf das damit verbundene landeskundliche Thema, sondern auch innerhalb der Einheiten nimmt Landeskunde bis zu einem Viertel der Aufgaben, verteilt über die gesamte Einheit, ein.

Am Ende jeder Einheit findet sich ein Quiz, das Faktenwissen zu der präsentierten Stadt abfragt. Des Weiteren bieten die kreativen Aufgaben (in >Differenzierung`) ein bis zwei Aufgaben, die explizit landeskundliche Inhalte zum Thema haben. So erstellen die Lernenden in Gruppen eine Broschüre oder ein Poster zu Hamburg, Köln oder Wien (NK1-2 A: 146), ein Brettspiel zu Dresden/ Leipzig, Frankfurt oder Bremen (NK3 B: 168) oder basteln einen Kalender mit Feiertagen aus den DACH-Ländern und den Niederlanden, in dem sie auch kurze Erläuterungen und Bilder ergänzen (NK3 A: 169). Landeskundliche Lernziele werden im Lehrwerk erstaunlicherweise nicht formuliert.

Auffallend in Na klar! ist, dass das Lehrwerk bereits auf Anfängerniveau (A1) literarische Texte, wie Kurzgeschichten und Gedichte, aufnimmt, meist im Teil >Differenzierung . Beispielsweise finden sich in dieser Kategorie ein Lesetext und ein You Tube-Trailer zum Wunder von Bern mit Verständnisfragen und ein Lautgedicht von Jandl, das die Lernenden sich online anhören und dazu Verständnisfragen beantworten können (NK3 A: 170f., vgl. Abbildung 18a und b). 


\section{Literatur |}

\section{Das Wunder von Bern}

\section{Familie Lubanski}

Unsere Familie wohnt in Essen. Aber Essen ist nicht irgendeine Stadt. Essen liegt nämlich mitten im Ruhrpott. Eigentlich heißt es „Ruhrgebiet", weil es hier einen Fluss gibt. Und das ist die Ruhr. Aber „Ruhrgebiet” sagen nur die vornehmen Leute. Doch wir sind nicht so vornehm. Wir sagen nicht Ruhrgebiet, sondern „Ruhrpott“ oder einfach nur „Pott”. Pott kommt von

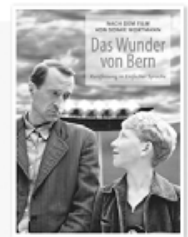
„Pütt“. So nennt man das Bergwerk, in dem die Bergleute arbeiten. Im Pütt ist die Kohle, Ganz tief unter der Erde, Vornehm ist nichts bei uns im Ruhrpott. Hier sind die Zechen. Dreck und Ruß. Rauch und Staub. Blauer Himmel, was ist das? Unsere Häuser sind schwarz vom Staub der Kohle. Ruß klebt auf den Straßen, an Türen und Wänden. Und sogar auf dem Weißkohl im Gemüsegarten. Bei uns im Pott sagt man statt „arbeiten“" „malochen“. Das ist nämlich viel härter als nur arbeiten. Wir sagen auch nicht wie die feinen Leute „das“ und „was“. Wir sagen „dat“ und „wat". Und wir essen, weil wir Kohldampf haben. „Kohldampf“ sagen wir, wenn wir Hunger haben. Hunger kennen wir noch vom Krieg. Aber jetzt haben wir nur noch Hunger vom vielen Malochen. Der Krieg ist zum Glück schon neun Jahre vorbei. Unser Ruhrpott geht von Duisburg bis Dortmund, und mittendrin liegt Essen. Genau da wohnt unsere Familie. Wir, die Familie Lubanski. Aber wir sind nicht irgendeine Familie. Wir sind keine normale Familie mit Vater, Mutter, Kind. Bei uns fehlt nämlich das Wichtigste: der Mann, der Vater, das Oberhaupt der Familie. Normal sitzt der Vater beim Essen immer an derselben Stelle. Am Kopf des Tisches. Da, wo man alles kontrollieren kann. Da, wo kein anderer sitzen darf. Nur das Oberhaupt der Familie darf da sitzen. Und das Oberhaupt ist immer der Vater. Bei uns ist das anders: Bei uns sitzt Benno am Kopf des Tisches. Benno ist der Älteste von uns drei Kindern. Benno ist schon 18. Er ist fast schon fast ein Mann. Benno lässt sich nicht mehr alles sagen. Auch nicht von unserer Mutter Christa, die ihm am Tisch gegenüber sitzt. Am anderen Ende des Tisches sitzt sie. Da hätte sich unser Vater Richard niemals hingesetzt. Damals, als er noch nicht verschwunden war. Damals, als Richard noch das Oberhaupt der Familie war. Jetzt sitzt sein Sohn Benno auf seinem Platz. Unsere Schwester Ingrid hört noch auf unsere Mutter. Obwohl Ingrid auch schon fast 17 ist. Ingrid ist verdammt hübsch. Sie sieht überhaupt nicht mehr aus wie ein Kind. Ingrid Lubanski sieht so klasse aus, dass die Kerle sich nach ihr umdrehen. Und dann ist da noch der Kleinste: Matthias Lubanski. Bei allen heißt er nur Matthes. Mit seinen elf Jahren sieht er die Welt noch mit Kinderaugen. Anders als sein Bruder Benno oder seine Schwester Ingrid. Und ganz anders als seine Mutter Christa. Matthes hat noch Träume. Matthes liebt Fußball. Rot-Weiß Essen. Das ist seine Mannschaft. Bei Rot-Weiß spielt Helmut Rahn. Für Matthes ist der ein Fußballheld. Matthes darf ihm die Tasche mit seinem Sportzeug tragen. Dann, wenn es zum Training geht. Für Matthes ist Fußball alles! Und wenn Rot-Weiß Essen verliert, darf keiner von uns eine blöde Bemerkung machen. So wie heute beim Abendessen: Mama betet mit uns das Tischgebet, und wir fangen an zu futtern. Nur Matthes nicht. Der reibt mit dem Finger auf dem Tisch herum. „Matthes, du musst was essen“, sagt Mama. „Hab keinen Hunger“, flüstert Matthes. „RotWeiß hat verloren.“ Mama lacht: „Die verlieren doch immer.“ Das ist zu viel für Matthes. Er steht auf, geht hinaus. Hinaus in den kleinen Garten, zum Kaninchenstall. Da krabbelt er hinein und erzählt seinen beiden Kaninchen von seinem Kummer: „1:o. Wir haben verloren. So werden wir nie deutscher Meister." Die beiden Kaninchen, Atze und Blacky, gehören auch zu unserer Familie. Für Matthes sind sie wie Seelentröster. Wenn er traurig ist, kriecht er zu ihnen in den Stall. Dann futtert er mit ihnen die alten Möhren. Und wenn er mit ihnen spricht, geht es ihm gleich viel besser, Bron: www.spassamlesenverlag.de (bewerkt)

$1 \rightarrow$ Höre dir das Fragment Das Wunder von Berm an. Lies den Text mit. Beantworte danach die Fragen.

1 Vul de ontbrekende woorden in.

Name der Familie:

Vater: ; Mutter: $\quad$; Kinder:

Wohnort: Fluss, der durch Essen fließt:

Fußballmannschaft des Sohnes: Haustiere:

Abbildung 18a: Literatur: Das Wunder von Bern (Na klar 3 A: 170) 
2 Bekijk het boekomslag. Wie zijn de twee personen?

3 Waarover gaat het verhaal?

4 Wanneer speelt het verhaal? Markeer de zin waar je het antwoord hebt gevonden.

5 Er wordt niet gezegd waar de vader is. Waar is hij nog niet terug van, denk je?

6 Das Wunder von Bern. Over wat voor wonder gaat het? Zoek het op internet op.

7 Bekijk de trailer van Das Wunder von Bern op www.youtube.com. Controleer of je antwoord op vraag 3 met de trailer overeenkomt.

\section{Ernst Jandl: ottos mops}

ottos mops trotzt otto: fort mops fort ottos mops hopst fort otto: soso otto holt koks otto holt obst otto horcht otto: mops mops otto hofft ottos mops klopft otto: komm mops komm ottos mops kommt ottos mops kotzt otto: ogottogott

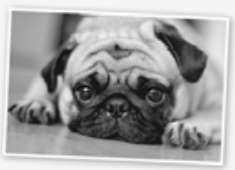

\section{Worthilfe}

trotzt is opstandig

hopst huppelt

horcht luistert

\begin{tabular}{l|l} 
kotzt spuugt, geeft over &
\end{tabular}

$2 \rightarrow$ Dieses Gedicht von Ernst Jandl ist ein Lautgedicht. Das bedeutet, dass man es hören muss, damit man es richtig versteht. Gehe auf die Site www.lyrikonline.org. Suche bei Autoren: Ernst Jandl. Höre dir das Gedicht ottos mops an. Ernst Jandl liest es selbst vor. Lies den Text mit.

$\rightarrow$ Arbeitet zu zweit. Beantwortet zuerst die Fragen. Lest das Gedicht danach abwechselnd laut vor.

1 Wat valt je op in dit gedicht?

2 Waarover gaat het gedicht? Geef de inhoud weer in het Nederlands.

3 Wat vond Ernst Jandl bij dit gedicht belangrijk, denk je?

Abbildung 18b: Literatur: Ernst Jandl (Na klar 3 A: 171) 
Landeskunde bietet das Lehrwerk implizit, d.h. ohne expliziten Vermerk `Landeskunde`, an. Meistens geht es um Texte mit Wissen über Land, Leute und Sprache in Form von bis zu einseitigen Lesetexten und Videos mit Spracharbeit mit anschließenden Lese- oder Hörverständnisfragen, z. B. über die zwei ehemaligen deutschen Staaten (NK3 A: 44), Frankfurt am Main (NK3 B: 63) oder die Wartburg und Martin Luther (NK3 B: 34). Auch gibt es verschiedene Beispiele, in denen Landeskunde mit Grammatik verbunden wird. Lückentexte zu touristischen Sehenswürdigkeiten in Bern (NK3 A: 118) und Bremen (NK3 B:122) werden beispielsweise als Grammatikübungen für Dativ und Akkusativ-Präpositionen bzw. Konjunktiv II verwendet. Auch werden Ampelmännchen zur visuellen Unterstützung bei der Grammatikvermittlung von Wechselpräpositionen eingesetzt (NK3 B: 14 und 17). Die Herkunft der Ampelmännchen wird allerdings nicht besprochen und so bleiben sie ein implizites Symbol aus Deutschland. Dies gilt ebenfalls für das Gemälde Der arme Poet von Carl Spitzweg; es dient ausschließlich dazu, die Wechselpräpositionen in einer gelenkten Schreibaufgabe (Bildbeschreibung) einzuüben (NK3 B: 39).

Landeskunde im Dienste einer Ausspracheübung ist zumindest ein Mal zu finden: ein Infotext zur Sächsischen Schweiz wird für das Training von ZischLauten eingesetzt, siehe Abbildung 19.

\footnotetext{
$\rightarrow$ Höre dir das Fragment Die Sächsische Schweiz an und lies den Text mit. Arbeitet zu zweit. Lest einander den Text vor und achtet auf die Aussprache.
}

\begin{abstract}
Bizarre Felsen - Wilde Schluchten: so sieht die Sächsische Schweiz aus. Die Sächsische Schweiz ist ein Gebirge aus Sandstein, das südlich von Dresden in Deutschland liegt und teilweise auch in Tschechien. In der Sächsischen Schweiz gibt es fantastische Felsen, die teilweise sehr hoch sind. Auch gibt es Schluchten, Felswände und Tafelberge. Daher wird die Sächsische Schweiz manchmal auch „der deutsche Grand Canyon“ genannt. Dieses Gebirge ist weltbekannt als Klettergebiet. Auch zum Wandern kommen viele Menschen in dieses Gebiet. In der Sächsischen Schweiz gibt es zahlreiche Tiere und Pflanzen: Es wachsen Buchen auf den Steinböden und im Wald wohnen Füchse und Dachse.
\end{abstract}

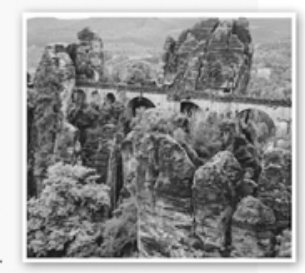

Abbildung 19: Die Sächsische Schweiz und Aussprache (Na klar 3B: 18)

Auch in Wortschatzübungen werden landeskundliche Phänomene für Sprachtraining eingesetzt, ohne die landeskulturellen Inhalte weiter zu thematisieren oder in Aufgaben aufzugreifen, z. B. in einem Text mit touristischen Infos zu Salzburg (NK3 A: 10) oder bei der Einübung der Monatsnamen: »Am 26. Oktober wird der österreichische Nationalfeiertag gefeiert." (NK3 A: 44). Landeskunde wird in $\mathrm{Na}$ klar! gelegentlich auch isoliert angeboten, wie in einem kurzen Informationstext über Köln, zu dem anschließend einzelne Fakten abgefragt werden (NK1-2 A: 57) und kognitives landeskundliches Wissen Ziel an sich zu sein scheint. 
Auch mittels name dropping erscheinen landeskundliche Inhalte implizit in $\mathrm{Na}$ klar!. So werden in E-Mails, Steckbriefen oder Chats, aber vor allem in Grammatikübungen Namen deutscher Städte (u.a. Potsdam, Lübeck, Gießen, Marburg), deutscher Fußballvereine (u. a. HSV, RB Leipzig), regionaler Gerichte und Getränke (u.a. Kaiserschmarrn, Almdudler, Apfelstrudel) und bekannter deutscher Gebäude (u. a. Sony-Center, Ulmer Münster) verwendet. Auch werden touristische Sehenswürdigkeiten wie der Bodensee oder Feste wie Züri Fäscht nebenbei in einer E-Mail aus dem Urlaub erwähnt (NK1-2: 146), und als Lösung eines Wortschatzrätsels erscheint der Name des Berliner Filmfestivals (NK1-2 B: 56). Ebenfalls erscheint der deutschsprachige Kulturraum manchmal implizit in Bildern, beispielsweise illustrieren beim Thema `Schweiz` und >Reisen`Fotos einer Swiss-Air-Maschine, eines Zugs der SBB und einer schweizerischen Straßenbahn eine Wortschatzübung, in der Konjunktionen ergänzt werden sollen (NK1-2 B: 141).

Mittels Abbildungen werden die landeskundlichen Themen für die Zielgruppe ansprechend visualisiert. Zeichnungen und Fotos von touristischen Sehenswürdigkeiten und meist jungen bekannten Deutschen, Österreichern und Schweizern, wie dem Sänger Johannes Strate (NK3 B: 125) oder dem Musiker Wincent Weiss (NK3 B: 70), erscheinen neben Texten und Aufgaben mit landeskundlichem Inhalt. Ebenfalls zu Aufgaben, in denen durch Nennung eines Ortsnamens (wie Graz, NK3 A: 23) implizit Landeskunde erscheint, wird manchmal beispielsweise durch ein Foto jenes Ortes Landeskunde auch implizit abgebildet. Gleiches erfolgt gelegentlich an Stellen, wo keine landeskundlichen Inhalte in Texten oder Aufgaben vermittelt werden, und vereinzelt ein Foto erscheint, das wiedererkennbar Deutschland, Österreich oder die Schweiz zeigt.

\subsubsection{Landeskundeansätze und Prinzipien in Na klar!}

\section{Vielfalt der deutschen Kultur- und Sprachlandschaft}

$\mathrm{Na}$ klar! zeigt die kulturelle und sprachliche Vielfalt des deutschsprachigen Raums, indem das Lehrwerk die einzelnen Einheiten thematisch mit verschiedenen Städten aus den DACH-Ländern verbindet. Schwerpunkt ist dabei das Nachbarland der Niederlande, Deutschland, aus dem acht der im Lehrwerk insgesamt zwölf vorgestellten Städte präsentiert werden. Inhaltlich fokussiert die Präsentation der einzelnen Städte auf die kulturellen Eigenheiten (z. B. Essen, Gebäude, Musiker). Die Plurilingualität der deutschen Sprache wird beispielsweise in regionalen Begrüßungen (Griass di, Servus, Grüß Gott, Pfiat di, Grüessechi, Uf widerluege, Ade) und mittels Speisen (halver Hahn, Schlagobers) sichtbar gemacht. Letzteres wird u. a. in einer Aufgabe thematisiert, in der die Bezeichnungen von Gerichten aus Deutschland und Österreich miteinander 
verbunden werden sollen (NK1-2 A: 110). Des Weiteren bietet das Lehrwerk drei Liedtexte, die in der Standardvariante der Schweiz (Palmen am Balkon von Granada; NK3 A: 18), in der österreichischen Standardvariante (Heimweh von Plüsch; NK3 A: 147) und in einem bayrischen Dialekt (Dorfkind von der Band Die Dorfrocker; NK3 B: 21) verfasst sind.

\section{Bezug zur Lebenswelt der Zielgruppe}

Sowohl in Design, Thematik als sehr wohl auch in den gewählten landeskundlichen Inhalten passt das Lehrwerk gut zu Jugendlichen in der Unterstufe niederländischer weiterführender Schulen (12-15 Jahre). Na klar! bietet Alltagskultur und fokussiert dabei stark auf Themen, die die Zielgruppe sehr wahrscheinlich interessieren. Es werden nicht nur die klassischen touristischen Sehenswürdigkeiten einer Stadt (Hamburg: Hafen, Brücken), sondern auch der Fußballverein HSV und die Musicalschule in Hamburg (NK1-2 A: 17), das Universum in Bremen (NK3 B: 126f.) und das Einkaufszentrum Arcaden in Köln (NK1-2 A: 70) präsentiert. Auch werden mittels Interviewtexten, Steckbriefen und Fotos vor allem junge bekannte Deutsche, Österreicher und Schweizer, die die jugendlichen Lernenden ansprechen sollten, wie Musiker (u.a. Cro), DJs (u.a. Felix Jaehn), Schauspieler und Sportler (u.a. Basketballspieler Dennis Schröder und Schauspieler Jannis Niewöhner und Elyias M’Barek) (NK3 B: 105 und NK1-2 B: 41) vorgestellt. Des Weiteren setzt Na klar! regelmäßig unter deutschen Jugendlichen populäre Fernsehsendungen wie Neuneinhalb, Die Mädchen-WG (in Band 1-2AB) und Die Jungs-WG (in Band 3AB) und ein Jugendbuch (Tschick) als (Hör)material in der digitalen Lernumgebung ein. Auch zeigt Na klar! den Lernenden, was ihre deutschen Altersgenossen bewegt: es werden deutsche Jugendliche in Steckbriefen, E-Mails und Blogs vorgestellt, in denen diese beispielsweise über ihre Hobbys, Sommerferien, ihr Taschengeld, ihren Schulweg berichten oder darüber, was sie persönlich für eine bessere Umwelt tun. Des Weiteren präsentiert jede Einheit zwei bis drei zum Thema passende Songtexte von bekannten Musikern oder Bands wie Gebt alles von Cassandra Steen (NK3 B: 141) und Wie schön bist du von Sarah Connor (NK3 B: 75) mit Foto und ein bis zwei Aufgaben (meist Wortschatz und Grammatik), die sich Lernende als Lied auf You Tube anhören können.

Darüber hinaus werden digitale Medien, die fest zum alltäglichen Leben niederländischer Jugendlicher gehören, regelmäßig eingesetzt, z. B. wenn die Lernenden aktiv im Internet recherchieren. So sollen sie beispielsweise gezielt Informationen zu Mode-Designern (NK1-2 A: 56), Erfindern (NK3 B: 144) und Festen und Feiertagen im deutschen Sprachraum (NK3 A: 143f.) suchen. Ein gelungenes Beispiel für integrative Landeskunde ist eine Aufgabe, in der Schüler selbstrecherchierte landeskundliche Informationen sprachlich weiterverwenden; die Lernenden recherchieren geographische Infos, tragen diese in eine 
Landkarte ein und wenden sie schließlich in Komparativsätzen an (NK1-2 B: 84f.).

Das Lehrwerk fördert die explizite Verknüpfung von angebotener Landeskunde mit der persönlichen Lebenswelt der Lernenden, wobei sich folgender Aufbau über die Lehrwerksbände hinweg feststellen lässt: In Band 1-2 wird ausschließlich persönliches Vorwissen über landeskundliche Inhalte aktiviert und nur in der ersten Aufgabe einer Einheit (`Orientierung`) Anschluss an die eigene Lebenswelt gesucht, z.B. »Warst du schon mal in einem deutschsprachigen Land? Wie viele Millionen Menschen sprechen Deutsch in Europa?« (NK1-2 A: 8) oder »Warst du schon mal in Salzburg? Was hast du da unternommen?« (NK3 A: 10). Nur vereinzelt wird auch nach dem persönlichen Geschmack gefragt, beispielsweise danach, ob die Lernenden ein Musikfragment mögen oder ob sie Zecki (aus dem Film Fack ju Göhte) gerne als Lehrer hätten und warum (NK1-2 B: 41). Ab Band NK3 A wird das landeskundliche Angebot deutlich persönlicher und vertiefender gestaltet: Lernende beschreiben ihre eigene Einstellung in Bezug auf ein präsentiertes Thema und müssen Stellung beziehen zu Äußerungen deutschsprachiger Altersgenossen, die das Lehrwerk in Interviews und Chats präsentiert. Die Lernenden werden beispielsweise dazu aufgefordert, zu berichten, ob und falls ja, welche Haustiere sie selbst haben (NK3 A: 87), was sie selber in Frankfurt gerne besuchen würden (NK3 B: 64), in welchen technischen Museen in den Niederlanden sie schon mal waren und welche Museen es am eigenen Wohnort oder in der Nähe gibt (NK3 B: 127) oder welches niederländische Buch sie einem deutschsprachigen Gleichaltrigen, der Niederländisch lernt, empfehlen würden und warum (NK3 B: 62). Obwohl die niederländische Ausgangskultur keine zentrale Position im Lehrwerk hat, wird sie auf diese Weise immer wieder in den Aufgabenstellungen berücksichtigt und ermöglicht somit einen Bezug zur Lebenswirklichkeit der Zielgruppe herzustellen.

\section{Stimulierung einer kulturreflexiven Haltung}

Ein direkter Vergleich zwischen der Ausgangskultur und der des deutschsprachigen Raumes findet sich in den Bänden 1-2 A und B kaum, und falls doch dann in aller Regel als vergleichendes Faktenwissen präsentiert, wie beispielsweise ein Informationstext zum deutschen und niederländischen Schulsystem (NK 1-2 B: 33). Im weiteren Verlauf des Lehrwerks, d. h. ab der zweiten Hälfte von Band 1$2 \mathrm{~B}$, werden die Lernenden häufiger dazu aufgefordert, die Informationen zur DACH-Alltagskultur mit der eigenen Ausgangskultur zu verbinden: »Mit welcher niederländischen Organisation kann man die schweizerische TCS vergleichen?« (NK 1-2 B: 138); "Kennst du niederländische Sänger/Bands, die über ihre eigene Stadt, Gegend oder eigenes Dorf singen? Welche?« (NK3 B: 22); »Kennt ihr solche Projekte [wie das vorgestellte Vivaldi-Projekt] auch in den 
Niederlanden? Werden an eurer Schule Musikprojekte organisiert? Nenne ein Beispiel.« (NK3 B: 88).

Landeskunde, die ausführlich und in mehreren Aufgaben thematisiert und verglichen wird, sind das Schulsystem, die Feiertage und der eigene Wohnort. So zeigt $\mathrm{Na}$ klar! einen kurzen Infotext zum heiligen Niklaus und einen dazu passenden Videobeitrag von Neuneinhalb mit anschließenden Textverständnisfragen und Fragen zur persönlichen Lebensrealität (NK3 A: 138f.). Auch wird das Niklausfest in Österreich, der Schweiz, Deutschland und den Niederlanden in einem Informationstext verglichen. Die Lernenden füllen eine Tabelle dazu aus und schreiben einen Blogeintrag über die eigene Einstellung zum Niklausfest (NK3 A: 142f.), machen Wortschatz- und Höraufgaben zu den christlichen Feiertagen und den Nationalfeiertagen in den Niederlanden und DACH-Ländern (NK3 A: 148f.) und sollen in einem Forumsbericht über das eigene niederländische Lieblingsfest (NK3 A: 158) schreiben. Die eigene Kreativität ist gefragt, wenn die Lernenden zu zweit für die Ausstellung »Wohnen in den Niederlanden« in einem Video oder einer Collage den eigenen Wohnort für Mitschüler präsentieren (NK3 B: 169).

In zwei Aufgaben werden die Lernenden gebeten, Stellung zu nehmen und auch die eigene Lebenswirklichkeit kritisch zu reflektieren: Nachdem die Lernenden in Form von einer E-Mail und einer Grafik über das österreichische Schulsystem informiert worden sind, sollen sie es mit dem niederländischen System vergleichen und für beide Länder zwei Vorteile nennen (NK3 A: $50 \mathrm{f}$.). Und, wie im Lehrwerk Neue Kontakte, vergleichen die Lernenden Mülltrennung in Deutschland und den Niederlanden: "Wie umweltbewusst sind die Niederländer? Wie werden in deinem Wohnort Abfälle getrennt? Was glaubst du, könnte man in den Niederlanden verbessern? « (NK3 A: 73). Aufgaben wie diese hinterfragen die eigene Perspektive und dürften eine offene, kulturreflexive Haltung fördern können. Die Schüler lernen auf diese Weise ein vermeintlich fremdes kulturelles Phänomen kennen. Sie dürften über die eigene Kultur kritisch-reflexiv zum Nachdenken angeregt werden und so auch auf ziel- und ausgangskulturelle Diskurse vorbereitet werden.

\section{Landeskunde erfahren}

$\mathrm{Na}$ klar! enthält keine Aufgaben, in denen Lernende Deutschland, Österreich oder die Schweiz direkt anhand von realen deutschsprachigen Personen oder Gegenständen aus dem deutschen Sprachraum erleben können. Es gibt allerdings vereinzelt Beispiele, in denen der eigene Raum zielsprachig aufgearbeitet wird, wie in der oben erwähnten Buchempfehlung. 


\section{Fazit}

Die deutschsprachige Alltagskultur nimmt eine zentrale Position in Na klar! ein. Jede Einheit hat eine Großstadt aus dem deutschsprachigen Raum als Bezugspunkt, mit der Themen wie Reisen, Schule und Freizeit verbunden werden, und bietet eine große Auswahl an Aufgaben mit landeskundlichem Inhalt. Touristische Sehenswürdigkeiten, bekannte deutschsprachige Musiker und deren Songtexte, Modedesigner, Schauspieler und das Leben deutschsprachiger Altersgenossen wird den niederländischen Jugendlichen in verschiedenen Formen vorgestellt. Meist wird Landeskunde in einem Hör- und Lesetext präsentiert, und es folgen Aufgaben mit Verständnisfragen, die in der Regel mit Training von Grammatik, Aussprache oder den produktiven Fertigkeiten verbunden werden.

Das Lehrwerk bietet ein ausgewogenes landeskundliches Angebot an Informationen aus verschiedenen DACH-Ländern. Die Ausgangskultur dagegen wird in $\mathrm{Na}$ klar! in Informationstexten und Bildern kaum thematisiert. In den Aufgaben werden die Lernenden allerdings regelmäßig dazu aufgefordert, ein DACH-Phänomen mit der eigenen Lebenswelt zu vergleichen. Festzuhalten ist, dass dabei ein kritischer Vergleich im Sinne eines kulturreflexiven Ansatzes zwar vereinzelt angeregt wird, aber letztlich doch eher Ausnahme bleibt.

\subsubsection{TrabiTour}

Das Lehrwerk TrabiTour besteht für die ersten zwei Jahre DaF-Unterricht aus zwei Textbüchern (TB1: A1-A2; TB2: A2-B1) mit jeweils drei Arbeitsbüchern: A, B, C und D, E, F. Insgesamt gibt es 18 Einheiten, dazu zählen auch drei Projekteinheiten zu Deutschland, Österreich und der Schweiz. Die Textbücher enthalten zu jeder Einheit Lesetexte, Fotos, Grafiken und eine Lernecke mit Grammatik und Wortschatz. Jeder Band bietet im Anhang Lerntipps, eine Grammatikübersicht, Wortschatz und Landkarten von den einzelnen DACHLändern. Ab Band 2 findet sich zusätzlich noch ein Abschnitt zu Jugendliteratur in den einzelnen Bänden (D, E, F). Leitend im Unterricht ist das Arbeitsbuch, das Aufgaben zu jeweils drei Einheiten enthält, mit einem Wiederholungsteil endet und auf Texte und Bilder im Textbuch und auf Hör- und Videomaterialien auf der Webseite von TrabiTour verweist. Auf der abgeschirmten Webseite zum Lehrwerk finden sich des Weiteren ein Grammatik- und Wortschatztrainer, Wimmelbilder, Karaoke-Fassungen zu den angebotenen Liedern, Webquests und Tests zum Üben.

In 18 Einheiten werden die Lernenden auf eine Reise durch den deutschen Sprachraum geführt und lernen dabei die deutsche Sprache und die Besonderheiten und Gewohnheiten der DACH-Länder kennen (TT TB1: 5). Band 1 enthält folgende Titel bzw. Themen: Grüße aus Deutschland (Deutschland); 
Aber bitte mit Sahne (Essen); Ich wünsch' dir was (Feste); Fantastische Freizeit (Hobbys); Bock auf Schule (Schule); Auf Schnäppchenjagd im Schlussverkauf (Geld); Hier bin ich zu Hause (Wohnort); Tierisch tierisch (Tiere); Servus aus Österreich (Österreich). Und Band 2 beinhaltet die Einheiten: Ich verstehe nur Bahnhof (Verkehr); Hals- und Beinbruch (Gesundheit); Hier geht die Post ab (Freizeit); Von App bis Zeitung (Medien); Schneegestöber und Hüttenzauber (Wintersport); Rein ins Rampenlicht (Film); Kleine Meister ganz groß (deutsche Marken); Leg dich in die Sommersonne (Sommerferien); Grüezi aus der Schweiz (Schweiz).

\subsubsection{Stellung und Art der Vermittlung von Landeskunde in TrabiTour}

Die Bedeutung, die TrabiTour der Landeskunde beimisst, erscheint auf den ersten Blick widersprüchlich. Einerseits ist die Kultur der deutschsprachigen Länder im Lehrwerk deutlich präsent. Das zeigen nicht nur der Name des Lehrwerks und das Buchcover, mit Collagen aus Fotos vom Prater, der Berliner Mauer, dem Brandenburger Tor, einem Fußball mit PUMA-Logo, Brezeln, Fachwerkhäusern und einem Trabant, sondern auch die Einführungen zu den einzelnen Einheiten. Mit bunten Bildern und werbendem Text wird den Lernenden zu Beginn jeder Einheit deutlich gemacht, was sie in der jeweiligen Einheit über Deutschland, Österreich und in geringerem Maße auch über die Schweiz erfahren werden.

Auch in der Lernstoffübersicht steht Landeskunde an erster Stelle, also vor Grammatik und den Fertigkeiten (TT TB1: 2f.). Des Weiteren werden darin Landeskunde und auch Hörverstehen mit landeskundlichem Inhalt farblich markiert ( $a b$ Band 2). Andererseits werden explizite landeskundliche Lernziele nur für drei Projekt-Einheiten (zu Deutschland, Österreich und zur Schweiz) formuliert.

In den Aufgaben ist Landeskunde immer prominent anwesend. So stehen am Anfang jeder Einheit drei Aufgaben mit landeskundlichem Inhalt, die auch als solche, also explizit, gekennzeichnet werden. Sie präsentieren Wissen über die DACH-Länder in Form von Hör- und vor allem Lesetexten mit Textverständnisfragen. Dann folgt eine Höraufgabe mit landeskundlichem Inhalt zum Thema der Einheit. Verteilt über eine Einheit gibt es noch zwei bis drei weitere Aufgaben, die Landeskunde implizit anbieten. Am Ende einer Einheit steht jeweils ein längerer Lesetext, der explizit als Landeskunde markiert wird. Der Text hat einen Bezug zum Thema der Einheit und präsentiert ein Phänomen aus den deutschsprachigen Ländern, das man in den Niederlanden in dieser Form nicht kennt. Insgesamt enthalten deutlich weniger als die Hälfte der Aufgaben landeskundliche Inhalte. In den Projekt-Einheiten, in denen Deutschland, Österreich und die Schweiz und deren Geschichte, Geographie, Sprache, Musik, Se- 
henswürdigkeiten, Nationalhymnen und Gewohnheiten zentral stehen, sind nahezu alle Aufgaben und Bilder mit landeskundlichem Inhalt verbunden. Auch der Wiederholungsteil enthält Landeskunde, und zwar in Form eines Quiz und eines Lesetextes mit Verständnisfragen.

Darüber hinaus findet sich implizite Landeskunde auch in Aufgaben, in denen Landeskunde in Fertigkeitsaufgaben erscheint, z. B. in denen Lernende die eigene Schule und das niederländische Schulsystem in einem selbsterstellten Video für deutschsprachige Gleichaltrige erläutern sollen (TT AB-B: 72). Oder sie recherchieren und sprechen über deutsche Marken (TT AB-F: 29ff.) und erstellen ein Poster zu einer selbstausgewählten deutschen Erfindung oder Marke (TT AB-F: 45).

Implizit erscheint Landeskunde auch, nämlich dann, wenn z. B. Ortsnamen, Flussnamen oder Namen von Fußballvereinen in Lesetexten, Interviews, EMails, Grammatikaufgaben oder in einer Ausspracheübung (Gelsenkirchen, Gießen, Goslar, Gronau, TT AB-B: 96) auftauchen, oder wenn Farbnamen anhand der Farben der österreichischen Staatsflagge geübt werden (TT AB-B: 91). Auch in Logos zeigt sich implizit der deutschsprachige Alltag, z.B. Lufthansa (TT AB-F: 68), Manner (TT AB-C: 82), Tagesschau (TT TB2: 55), Deutsche Bahn (TT TB2: 13) und Kamps Backstube (TT TB2: 36) sowie in Bildern von Warenhäusern und Supermärkten, z. B. KaDeWe (TT AB-B: 79), Lidl (TT AB-B: 80) und Edeka (TT AB-B: 87).

Es finden sich zahlreiche Illustrationen im Lehrwerk, von denen ein Teil landeskundliche Inhalte abbildet. Fotos und Grafiken präsentieren mit dazugehörigen Aufgaben den deutschsprachigen Raum, wie Abbildung 20 zeigt. Sie passen zu Aufgaben mit landeskundlichem Inhalt oder zu Grammatikübungen, in denen z.B. ein deutschsprachiger Ortsname oder Begriff genannt wird. Die Mehrzahl der Fotos im Arbeitsbuch weist keinen landeskundlichen Bezug auf. Es betrifft dann nichtssagende Bilder mit oft jungen Menschen, was wohl zur Zielgruppe passt. 


\section{LANDESKUNDE}

2 Deutsch auf Bildern I
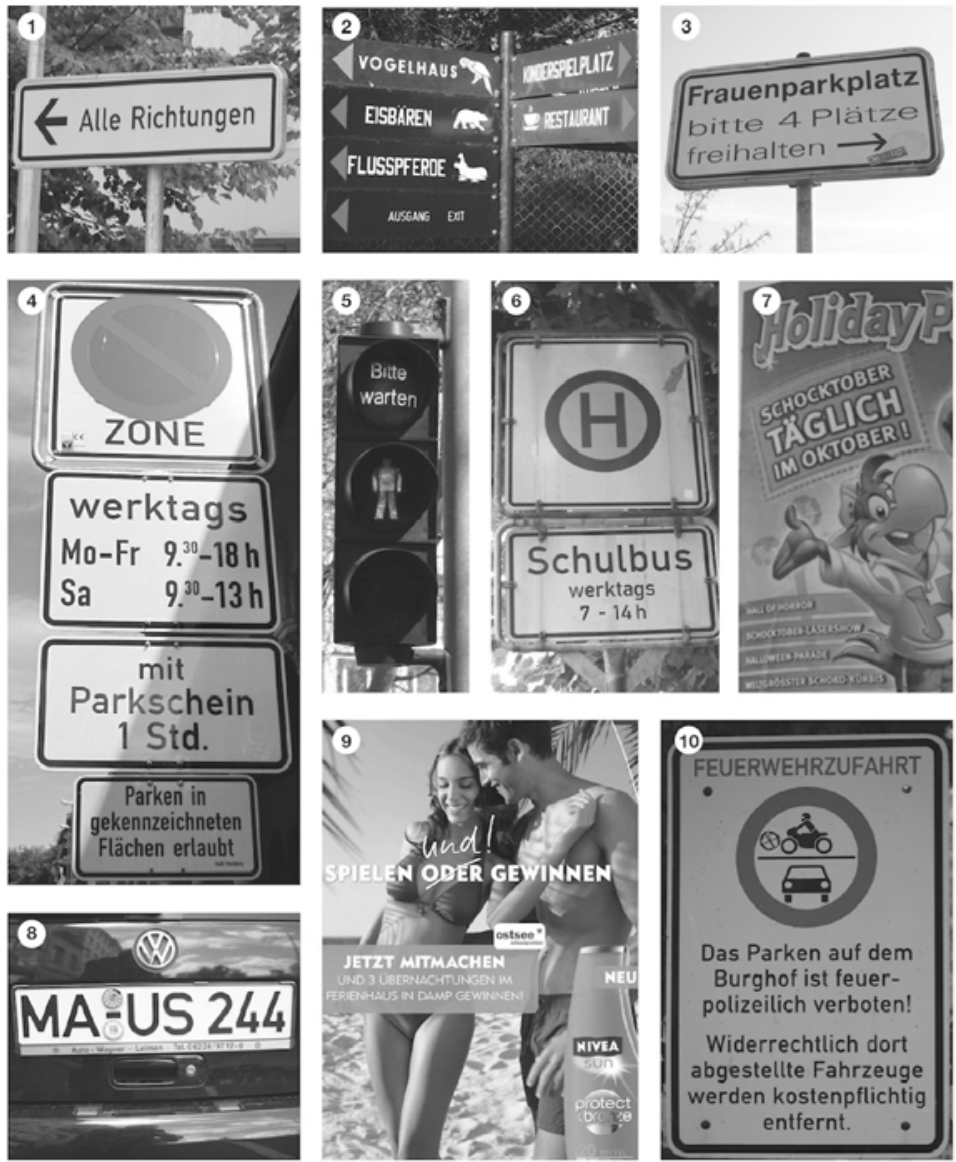

(2)

Abbildung 20: Deutsch auf Bildern (TrabiTour TB1: 6) 


\subsubsection{Landeskundeansätze und Prinzipien in TrabiTour}

Vielfalt der deutschen Kultur- und Sprachlandschaft

Das Lehrwerk zeigt eine breite Skala an kulturellen Phänomenen; es präsentiert Deutschland, Österreich und die Schweiz durch deren Musik, Produkte, Sportler, Sportarten, Erfinder und Geschäfte. Obwohl TrabiTour dabei auf die Alltagskultur fokussiert, fällt auf, dass auch viele Informationen und Aufgaben zur Geschichte und Geographie des deutschsprachigen Raumes angeboten werden. Die Mehrzahl der Beiträge mit kulturellem Inhalt bezieht sich auf Deutschland, obwohl auch Österreich und die Schweiz vertreten sind, sei es auch vereinzelt klischeehaft (Sissi, Sachertorte). Die sprachliche Vielfalt des deutschsprachigen Raumes wird in zwei Projekt-Einheiten erkennbar; eine zu Österreich (in Band C) und eine zu der Schweiz (in Band F). Beide erwähnen Begrüßungen (Servus, Grüezi) und bieten eine Aufgabe, die Wörter und Redewendungen aus Österreich bzw. der Schweiz mit dem Hochdeutschen vergleicht (Erdäpfel, heuer, Feber; Goht's no? Frage chostet nüt, Velo). Bei einem Lied aus Österreich (I sing a liad für di von Andreas Gabalier) und zwei aus der Schweiz (Samichlaus-Spruch und Nationalhymne) werden die Lernenden gebeten, sich diese anzuhören und Teile ins Niederländische zu übersetzen.

\section{Bezug zur Lebenswelt der Zielgruppe}

Die allgemeinen Themen in TrabiTour beschreiben, wie auch in Neue Kontakte und Na klar!, mehrheitlich die Alltagskultur und passen zur Lebenswelt der Zielgruppe. Die präsentierten landeskundlichen Inhalte lassen sich grob in drei Bereiche aufteilen: (i) für die meisten wahrscheinlich unbekannte Sehenswürdigkeiten oder Phänomene mit Kuriositätscharakter, wie das Currywurstmuseum (TT TB1: 23) und der Hüttenpalast in Berlin (TT AB-F: 57) oder das Faltrad-Abo in Münster (TT TB2: 10); (ii) kulturelle Phänomene, die es so in den Niederlanden nicht gibt, wie Polterabend (TT TB1: 39), Jugendschutzgesetz (TT TB2: 43), die mündliche Note (TT TB1: 59), Sternsinger (TT TB1: 68) oder Umweltplaketten (TT AB-D:38) und (iii) was die deutsche Jugend bezüglich z. B. Mediennutzung, Taschengeld und Wohnen beschäftigt, übermittelt mittels Grafiken, Interviews, E-Mails und Dialogtexten. Vor allem diese letzte Kategorie bezieht sich direkt auf die Lebenswelt der niederländischen Schüler; bei den anderen Bereichen ist der Bezug weniger einleuchtend, und die Themen sind nicht immer altersgerecht.

Sehr wohl passend zur Zielgruppe sind die Teile mit explizit angebotener Landeskunde; darin erhalten die niederländischen Lernenden Informationen über das Leben ihrer Altersgenossen in Deutschland, Österreich und der Schweiz. Das Lehrwerk bietet dazu Hör- und Lesetexte zu für Jugendliche potentiell interessanten Events und Phänomenen im deutschen Sprachraum, wie 
etwa dem Europa Park in Rust (TT AB-F: 50), dem CentrO (TT TB1: 77), der Gamescom in Köln (TT TB2: 57), dem Freien Radio (TT AB-E: 7 f.) oder deutschen Filmen (TT TB2: 87) an. Als Quellen werden regelmäßig unter deutschen Jugendlichen bekannte Zeitschriften wie GEOlino, Dein Spiegel und Yuno verwendet, auch wird auf deutsche Fernsehsendungen wie Elefanten, Tiger \& Co. verwiesen.

Eine weitere Möglichkeit, einen Bezug zur Lebenswelt der Jugendlichen herzustellen, nutzt TrabiTour, indem gelegentlich versucht wird, zu den landeskundlichen Inhalten einen persönlichen Zugang zu ermöglichen. So werden die Lernenden beispielsweise dazu angeregt, die präsentierten landeskundlichen Inhalte mit ihrer eigenen (persönlichen) Situation in Zusammenhang zu bringen oder sollen diese miteinander vergleichen oder dazu Stellung nehmen: »Würdest du auch gerne an einem `Musikcamp $\prec$ bei den Salzburger Festspielen teilnehmen?" (TT AB-C: 88), "Was möchtest du [in Zürich] unbedingt sehen und warum?» (TT AB-F: 96) oder »Welche Erfindung findest du am wichtigsten?« (TT AB-F: 38). Was in TrabiTour des Weiteren auffällt, sind zahlreiche kreative Aufgaben und Rätsel mit landeskundlichem Inhalt, die zum affektiven Lernen beitragen dürften. Landeskunde wird für die Lernenden zugänglich und attraktiv präsentiert, indem sie - wenn auch vereinzelt stark klischeehaft belastet - z. B. eine Sachertorte backen (TT AB-C: 94f.), ein Hundertwassergebäude oder einen neuen Slogan mit Logo für eine Österreich-Werbekampagne entwerfen (TT AB-C: 96f.), Sissi-Szenen nachspielen (TT AB-C: 92f.) oder einen eigenen Berliner Bären basteln sollen (TT AB-A: 27).

Ein gelungenes Beispiel für ein Rätsel bietet Abbildung 21, bei dem Lernende mit acht Hinweisen herausfinden sollen, wer welchen Kuchen gebacken hat (TT AB-A: 54), oder ein Bundesligalogo-Sudoku lösen können (TT AB-B: 23). Sehr oft werden diese kreativen Aufgaben allerdings als Extra-Stoff gekennzeichnet, der nur, wenn die Unterrichtszeit es zulässt, zum Einsatz kommen dürften.

Regelmäßig recherchieren die Lernenden selbst und zielgruppengerecht, im Internet oder - zumindest auf den ersten Blick, sicher für diese Zielgruppe, etwas altertümlich anmutend - im Atlas. So suchen sie beispielsweise die Namen von deutschen Mobilanbietern (TT AB-E: 8), Infos zur Geschichte und Geographie von Österreich (TT AB-C: 77) oder erkundigen sich über deutsche Bands und deren Hits (TT AB-E: 95f.).

TrabiTour versucht somit zweifelsohne, einen Bezug zur Lebenswelt der niederländischen Schüler herzustellen. Zu konstatieren ist jedoch, dass dies sowohl in Bezug auf die gewählten landeskundlichen Themen (wie Sissi) als auch bezüglich der vorgeschlagenen Recherche-Möglichkeiten (mit Atlas) nicht immer zielgruppengerecht geschehen dürfte. Gleichzeitig kann aber davon ausgegangen werden, dass vor allem die vielen Puzzles, Rätsel und Bastelaufgaben kreative Lernprozesse stimulieren. 


\subsection{Rätsel}

Op het Einstein-Gymnasium wordt een fancy fair gehouden. Alle leerlingen doen mee. De opbrengst gaat naar een kindertehuis in Roemeniē. Katja, Laura, Ariane en Kübra uit klas $8 \mathrm{a}$ hebben alle vier een verschillende taart gebakken. De taartpunten verkopen ze alle vier op de fancy fair voor een verschillende prijs. Probeer met behulp van de volgende aanwijzingen uit te vinden wie van de meiden welke taart heeft gebakken en wat een stukje van de taart kost. Viel Spaß und viel Erfolg!

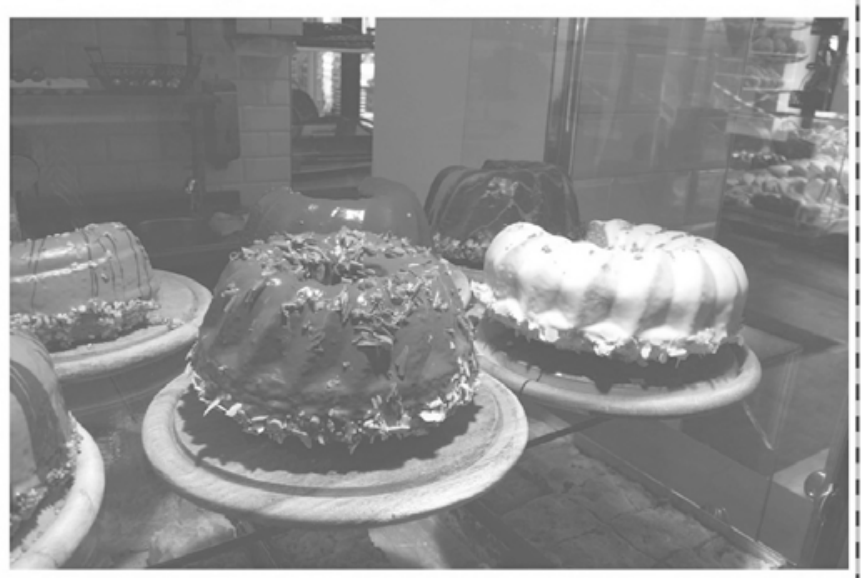

Streep met behulp van de aanwijzingen steeds foute antwoordmogelijkheden weg, totdat alleen het goede antwoord overblijtt.

1. Katjas Kuchen kostet nicht $€ 2,00$.

2. Ariane hat keinen Apfelstrudel gebacken.

3. Ein Stück Schwarzwălder Kirschtorte kostet $€ 2,00$.

4. Kübra hat nicht den billigsten (goedkoopste) und auch nicht den teuersten (duurste) Kuchen gebacken.

5. Laura hat den Schokoladenkuchen gebacken.

6. Ein Stück Apfelstrudel kostet $€ 2,50$.

7. Kübra hat nicht den Erdbeerkuchen gebacken.

8. Der Kuchen von Laura ist teurer (duurder) als der Kuchen von Ariane.

\begin{tabular}{|c|c|c|c|}
\hline Katja & Laura & Ariane & Kübra \\
\hline Schwarzwalider Kirschtorte & Schwarzwalider Kirschtorte & Schwarzwalder Kirschtorte & Schwarzwalider Kirschtorte \\
\hline Erdbeerkuchen & Erdbeerkuchen & Erdbeerkuchen & Erdbeerkuchen \\
\hline Apfelstrudel & Apfelstrudel & Apfeistrudel & Apfelstrudel \\
\hline Schokoladenkuchen & Schokoladenkuchen & Schokoladenkuchen & Schokoladenkuchen \\
\hline$€ 1,75$ & $€ 1,75$ & $€ 1,75$ & $€ 1.75$ \\
\hline$€ 2,00$ & $€ 2,00$ & $€ 2,00$ & $€ 2,00$ \\
\hline$€ 2,25$ & $€ 2,25$ & $€ 2,25$ & $€ 2,25$ \\
\hline$€ 2,50$ & $€ 2,50$ & $€ 2,50$ & $€ 2,50$ \\
\hline
\end{tabular}

Abbildung 21: Rätsel Kuchen (TrabiTour AB-A: 54) 


\section{Stimulierung einer kulturreflexiven Haltung}

Regelmäßig wird in den landeskundlichen Informationstexten auf Unterschiede zwischen den Niederlanden und dem deutschen Sprachraum hingewiesen, wobei Phänomene aus dem deutschsprachigen Raum vorgestellt werden. So werden die Lernenden über für sie unbekannte Institutionen oder anders tradierte Gewohnheiten und Gepflogenheiten wie etwa Kaffee und Kuchen, die Schulkantine, Frühstück oder Duzen und Siezen informiert. Explizite Vergleiche gibt es auch in Infotexten, wie etwa: »In Deutschland gibt es auf 1000 Einwohner zweimal so viele Hausärzte wie in den Niederlanden « (TT TB2:29) oder »Anders als in den Niederlanden werden in Deutschland abends meistens die Vorhänge und Rollläden geschlossen« (TT TB1: 91). Explizit verglichen werden auch Einwohnerzahl und Bevölkerungsdichte der Niederlande mit der Schweiz (TT AB-F: 93), deutsche und niederländische Apotheken (TT AB-D: 49), das niederländische Sinterklaas-Fest mit dem Samichlaus in der Schweiz (TT AB-F: 98f.) und die niederländische und deutsche Tastatur (TT AB-E: 8f.). Manchmal wird etwas im deutschsprachigen Raum mit etwas Ähnlichem in den Niederlanden verglichen, wie das Miniaturwunderland in Hamburg mit Madurodam in Den Haag (TT AB-F: 30) oder Lernende werden selbst zu Vergleichen angeregt: "Gibt es in den Niederlanden auch so eine Art Zeitung [wie die BILD-Zeitung]?» (TT AB-E: 5), oder leicht stereotypisierend: »Was sieht man auf dem Bild an der Ostseeküste bei Usedom, was man an den niederländischen Stränden nie sieht?» (TT AB-F: 47) oder »Würdest du einen Strandkorb mieten? Warum?« (TT AB-F: 48).

In den Landeskundeaufgaben werden Lernende regelmäßig dazu aufgefordert, ihre Meinung über das, was sie gerade über die vermeintlich ıfremder Kultur gelernt haben, zu äußern, beispielsweise bei der Frage: »Was meinst du? Ist man mit 16 alt genug zum Wählen [wie in Österreich] oder nicht? Begründe deine Meinung auf Niederländisch.«(TT AB-C: 84). Dass das Lehrwerk sich hier für Niederländisch entscheidet, ist nicht verwunderlich; die Deutschkenntnisse der Lernenden dürften nach knapp einem Jahr Unterricht noch nicht ausreichen, ihre Ansichten zu komplexeren Themen wie diesen schriftlich oder mündlich auf Deutsch zu erläutern.

In einigen Fällen, wie z.B. zum Thema mündliche Note (TT AB-B: 56) oder festes Klassenzimmer (TT AB-B: 39), werden die Lernenden aufgefordert, auch Vor- und Nachteile zu benennen und somit unterschiedliche kulturelle Phänomene zu reflektieren. Solche Aufgaben dürften die Lernenden inhaltlich (aber nicht unbedingt auch sprachlich) auf den fremden Diskurs vorbereiten.

\section{Landeskunde erfahren}

In zwei Mini-Projekten werden die Lernenden mit realen Gegenständen aus dem deutschsprachigen Gebiet konfrontiert. In einem davon sollen sie deutsche 
Zeitungen, die am Bahnhofskiosk größerer niederländischer Städte erhältlich sind, kaufen und miteinander vergleichen (TT AB-E: 15). Im zweiten Projekt sehen sich die Lernenden zu zweit einen deutschen Film mit deutschen Untertiteln an und berichten darüber anschließend in der Klasse (TT AB-E: 107).

\section{Fazit}

TrabiTour bietet den Lernenden ein breites Spektrum an Alltagskultur und fokussiert dabei auf Faktenwissen, das in Lese- und Hörtexten vermittelt wird. So wird z. B. das deutsche Notensystem erläutert, anschließend folgen Reproduktionsfragen, wie etwa: was ist die beste deutsche Note? Das Lehrwerk folgt dabei einem kontrastiv-orientierten Ansatz und betont die Unterschiede zwischen Ausgangs- und Zielkultur. Kulturreflexives Lernen wird dabei nur gelegentlich initiiert. Dabei ist anzumerken, dass des Öfteren recht klischeehafte landeskundliche bzw. klassisch touristische Inhalte präsentiert werden (Sachertorte, Sissi usw.), die zumindest nicht unbedingt etwas mit der Lebenswelt von DACHJugendlichen zu tun haben dürften. Auch auf sprachlicher Ebene wirken zumindest Einheitsüberschriften oft gewollt cool bzw. jugendsprachlich, dürften aber häufig nicht mehr aktuell (wie >Bock auf Schule « und `Hier geht die Post ab`) und entsprechend für Jugendliche nicht mehr ansprechend sein. Neben dem Fokus auf den Alltag im deutschsprachigen Raum fallen in TrabiTour zwei weitere Besonderheiten auf: Beiträge in Texten und Aufgaben im Sinne eines faktischen Landeskundeansatzes mit Bezug auf Geschichte und Geographie sowie ideenreiche Lernimpulse in Form von Rätseln sowie zahlreichen kreativen Aufgaben mit landeskundlichem Inhalt, wobei zu diesen anzumerken ist, dass sie im Lehrwerk selbst oft (nur) als optional eingestuft werden.

Explizites Wissen über die Niederlande oder Bilder und Grafiken der Ausgangskultur spielen keine Rolle im Lehrwerk: nur ein einziges Mal taucht eine Ansichtskarte mit Grachtenbild auf, dies dann mit der Aufgabenstellung, einem deutschen Bekannten die eigene Wohnlage zu beschreiben (TT AB-C: 32).

\subsubsection{Fazit: Niederländische Lehrwerke}

Die drei analysierten niederländischen Lehrwerke fokussieren auf den Alltag im deutschen Sprachraum, wobei jedes Lehrwerk unterschiedliche Konzepte und Ansätze zur Landeskunde anbietet und Schwerpunkte setzt (z.B. TrabiTour: Geschichte und Geographie; Neue Kontakte: Sprachenlandschaften im Sinne von plurilingualen Varianten und Na klar! die persönliche Verbindung zwischen der vermeintlich fremden und der eigenen Kultur). Letztlich lässt sich für die niederländischen Lehrwerke festhalten, dass sich in der Präsentation von landesund kulturkundlichen Inhalten kaum ein Aufbau feststellen lässt. Vermutlich ist 
dies der Fall, da in den Niederlanden curriculare Vorgaben zu Landeskunde fehlen und keine Richtlinien zu den landes- und kulturkundlichen Inhalten und deren Progression existieren. Nur so lässt sich erklären, wie innerhalb einer Einstiegslektion in einem Lehrwerk nach der Vorstellung des Brandenburger Tors plötzlich deutsche KFZ-Kennzeichen zum Thema gemacht werden, gefolgt von einem Songtext, nämlich Schnappi (dem Krokodil), abgeschlossen durch Sachertorte aus Österreich (vgl. TrabiTour Einheit 1), deren Auswahl von Autoreninteressen abhängig zu sein scheint und eine Progression landes- und kulturkundlicher Inhalte nicht erkennen lässt.

Darüber hinaus zeigen die vorliegenden Analysen, dass landeskundliche Inhalte mehrheitlich in Lesetexten mit Textverständnisfragen vermittelt werden. Auch dies dürfte ebenfalls auf den Kontext des niederländischen Fremdsprachenunterrichts zurückzuführen sein, und zwar vor allem auf den Einfluss der niederlandeweit verbindlich zentral durchgeführten Abschlussprüfungen, die zu mindestens 50 \% rezeptives Leseverständnis für das Prüfungsfach Deutsch beinhalten, zurückzuführen sein (vgl. Tammenga-Helmantel/Jentges 2016; Jentges/ Sars 2017).

\subsection{Landeskunde in polnischen Lehrwerken}

Die polnische Bildungslandschaft ${ }^{21}$ ist zurzeit in großem Umbruch. In Polen trat 2017 eine weitgehende Bildungsreform in Kraft, die u. a. die zuletzt im Jahr 1999 eingeführten Veränderungen im Bildungssystem rückgängig machte. Infolge der Reform wurde die 6-jährige Grundschule wieder um zwei Jahre verlängert, die dreijährige Mittelschule (gimnazjum) wurde abgeschafft und das dreijährige allgemeinbildende Lyzeum (eine Entsprechung des deutschen Gymnasiums) wieder von drei auf vier Jahre verlängert. Das polnische Bildungssystem ist in drei aufeinander aufbauende Stufen unterteilt. Die vorschulische Erziehung der Kinder im Alter zwischen drei und sechs Jahren erfolgt in Einrichtungen wie Kindergärten oder Vorschulabteilungen an den Grundschulen. Für 6-Jährige gilt eine Schulvorbereitungspflicht in der sogenannten Nullklasse (zerówka). Die eigentliche Bildungspflicht beginnt im Schuljahr, in dem das Kind sieben Jahre alt wird und dauert bis zum 18. Lebensjahr. Die reformierte Grundschule umfasst derzeit wieder acht Jahre. In den ersten drei Klassen begleitet meist eine Lehrkraft die Schüler in der Früherziehung. Ab der vierten Klasse setzen dann eigenständige Fächer wie Polnisch, Mathematik oder Geschichte ein. Nach der achten Klasse legen alle Schüler Prüfungen ab, deren Ergebnisse die Wahl der weiterführenden Schule weitgehend bestimmen.

21 Für die Organisation des Schulwesens in Polen ist das Bildungsministerium zuständig. 
Der Übergang in eine weiterführende Schule erfolgt in Polen im Alter von ca. 15 Jahren. Zu weiterführenden Schulen gehören zweistufige Branchenschulen (szkoła branżowa), fünfjährige technische Fachschulen (technikum) und vierjährige allgemeinbildende Lyzeen (liceum ogólnokształcace) ${ }^{22}$.

Die Lernziele und das zu erreichende Niveau bei der Vermittlung der Fremdsprachen sind, ähnlich wie im Falle von anderen Fächern, in den Rahmenlehrplänen fest verankert. Die erste Fremdsprache wird bereits ab der ersten Klasse der Grundschule mit zwei Unterrichtsstunden pro Woche vermittelt, die zweite Fremdsprache als Pflichtfach ab Klasse sieben mit ebenfalls zwei Stunden pro Woche; für die erste Fremdsprache sind ab Klasse sieben drei Stunden pro Woche vorgesehen. Es gibt landesweit auch Schulen, die eine zweite Fremdsprache bereits in der ersten oder vierten Klasse der Grundschule einführen. Solche Fälle sind jedoch sowohl aus finanziellen Gründen ${ }^{23}$, als vor allem auch aufgrund eines Mangels an entsprechend ausgebildeten Lehrkräften eher selten. Als erste Fremdsprache wird nahezu flächendeckend Englisch eingeführt. Deutsch profitiert aber von der Einführung der zweiten Pflichtfremdsprache und nimmt unter den Fremdsprachen mit einem Anteil von $40 \%$ Platz 2 nach Englisch (90\% der Schüler lernen Englisch) ein. Insgesamt lernen in einem Staat von ca. 38 Mio. Einwohnern zurzeit (Stand 2015) ${ }^{24}$ knapp 2,3 Mio. Menschen Deutsch, davon allein 2,1 Mio. an den Schulen.

Die Zulassung der Lehrwerke erfolgt landesweit und wird vom Bildungsministerium über Gutachter geregelt. Im Schuljahr 2017/2018 sind für die 7. Klasse, also für die Stufe, in der die zweite Fremdsprache als Pflichtfach ${ }^{25}$ regulär unterrichtet wird, 12 Titel für DaF zugelassen. Hierbei handelt es sich um Lehr-

22 Die dreijährige Branchenschule entspricht ungefähr der Berufsschule im deutschen Schulsystem. Die Bildung in der Branchenschule ist zweistufig aufgebaut. Das Abitur wird Branchenschülern ermöglicht, die nach drei Jahren (I. Stufe) noch eine zweijährige Branchenschule der II. Stufe absolvieren. In technischen Fachschulen können die Schüler Abitur ablegen, sie erwerben außerdem während ihrer Ausbildung eine Berufsqualifikation. Das Lyzeum entspricht ungefähr einem deutschen Gymnasium und endet mit dem Abitur.

23 Vom regulären Lehrplan abweichende Bestimmungen, die zusätzliche personelle und damit finanzielle Ressourcen erfordern, bedürfen der Genehmigung der zuständigen Behörde. Von dem Etat, der den Schuldirektoren zur Verfügung steht, müssen unterschiedliche zusätzliche Ausgaben finanziert werden. Oft ist es die Betreuung unterschiedlicher fakultativer AGs und sehr selten die Einführung einer zweiten Fremdsprache.

24 https://www.goethe.de/resources/files/pdf37/Bro_Deutschlernerhebung_final2.pdf.

25 Deutsch wird in der Regel in Polen erst als zweite Fremdsprache nach Englisch in Klasse 7 begonnen. $\mathrm{Zu}$ den häufig angebotenen Fremdsprachen gehören außerdem Französisch, Russisch sowie Italienisch. In den letzten Jahren ist der Trend zu Spanisch als zweite Fremdsprache an Schulen sichtbar. (vgl. https://ec.europa.eu/eurostat/statistics-explained/index.php?title=Fi le:Proportion_of_pupils_in_primary_education_learning_foreign_languages,_by_language,_2 016_(\%25)_ET18.png) 
werke für Schüler, die den Deutschunterricht fortsetzen ${ }^{26}$ als auch für diejenigen, die mit Deutsch erst anfangen. Zu den vor Ort produzierten Lehrwerken polnischer Autorenteams gehören Mit links!; www.weiter deutsch; Klasse! Super! Toll!; aha! Neu; Aktion Deutsch; Kompass Team; Das ist deutsch! Kompakt; Meine Deutschtour. Vier Titel stellen regionalisierte Ausgaben globaler Lehrwerke dar: Magnet; Magnet smart; Wir smart; Beste Freunde.

Für die vorliegende Analyse wurden drei gegenwärtig eingesetzte Lehrwerke unterschiedlicher Verlage und Autorenteams gewählt.

\subsubsection{Mit links!}

Das Lehrwerk Mit links! richtet sich an Schüler und Schülerinnen der 7. und 8. Klasse und ist konform mit den seit 2017 geltenden curricularen Richtlinien. Das Lehrwerk setzt sich aus zwei Bänden zusammen, die jeweils in Lehrbuch und Arbeitsbuch unterteilt sind. Dem Lehrbuch ist eine MP3-CD mit Hörtexten beigefügt. Das Anfängerlehrwerk führt nach dem GER zum A1-Niveau. In der vorangestellten Inhaltsübersicht im Lehrbuch werden die Lernziele der Einheiten nach Wortschatz, Grammatik und Kommunikation (Sprachhandlungen) untergliedert angegeben. Sowohl das Kursbuch als auch das Arbeitsbuch haben einen transparenten Lektionsaufbau. Pro Band gibt es 5 Einheiten mit jeweils 7 Lektionen, in denen folgende Themenbereiche aufgegriffen werden:

Band 1: Hallo!; Taschengeld; Schule; Meine Welt; Familie,

Band 2: Alltag; Mein Wohnort; Essen; Gesundheit; Reisen.

Jede Seite entspricht einer Lektion und ist für eine 45-minütige Unterrichtseinheit vorgesehen. Außer Übungen zur Vermittlung von Wortschatz, Redemitteln und Grammatik enthält jede Einheit einen `Schreiblink`, eine Lehrbuchseite mit kurzen, das Schreiben vorbereitenden Übungen und einen `Kommunikationslink mit Wortschatz und Redemitteln (deutsch-polnisch) aus der jeweiligen Einheit. Im Anhang der beiden Bände befinden sich außerdem folgende Seiten: >Grammatik kompaktı (ML KB 1: 63-68, ML KB 2: 61-66) mit Grammatikübersichten, `Kulturlink mit Lesetexten und dazugehörigen Übungen und/oder Aufgaben (ML KB 1:69-74, ML KB 2:67-72), eine Liste der unregelmäßigen Verben (ML KB 1:79, ML KB 2: 77) und eine alphabetisch geordnete (deutsch-polnisch) >Wortschatzlister (ML KB 1: 80-85, ML KB 2: 78-87).

$26 \mathrm{Zu}$ den Lehrwerken für fortgesetzten Unterricht gehören www.weiter.deutsch; Kompass Team Band 2 und Band 3; Wir smart Band 4 und Band 5. 
Beide Arbeitsbücher (zu Band 1 und Band 2) sind integraler Teil des Lehrmaterials und bieten vertiefende und/oder Wiederholungsaufgaben zu den Inhalten im Lehrbuch. Jeder Seite des Lehrbuchs entsprechen hier im Arbeitsbuch zwei Seiten mit Übungen zu Wortschatz und Grammatik. Jede Einheit enthält abschließend zusätzlich eine Doppelseite, die der Wiederholung dient und mit einer »Ich kann-Spalte« zur Selbstevaluation in Bezug auf Spracherwerb endet. Am Ende der Arbeitsbücher gibt es jeweils vier Seiten mit Übungen zu den `Kulturlink«-Seiten im Kursbuch (ML AB 1: 74-77, ML AB 2: 74-77). Im Arbeitsbuch werden auch Grammatikübungen zu den Regeln im Kursbuch (vgl. `Grammatiklink `) angeboten (ML AB 1: 78-86, ML AB 2: 78-87). Band 1 enthält außerdem eine Tabelle mit einundzwanzig unregelmäßigen Verben (ML AB 1: 87).

\subsubsection{Stellung und Art der Vermittlung der Landeskunde in Mit links!}

Die Stellung und Art der landeskundlichen Inhalte ist in diesen beiden Bänden vergleichbar. Sowohl in den Kursbüchern als auch in den Arbeitsbüchern werden die landeskundlichen Inhalte implizit eingebracht. Die Inhalte in Band 1 erschöpfen sich in allgemeinen Informationen wie die Höhe des Taschengeldes oder Preise von kleinen Elektrogeräten in Deutschland. Band 2 bietet weiterführende landeskundliche Informationen an, beispielsweise wird Lena Meyer Landrut als Gewinnerin des Eurovision Song Contest im Jahr 2010 vorgestellt (ML KB 2:6, 7). Daran knüpfen im Arbeitsbuch zwei >Weblinks` an, in denen die Lernenden dazu angeregt werden, zu den angegebenen Musikbands oder Sängern Informationen zu finden (ML AB 2:5) sowie die Auszeichnungen ermitteln sollen, die in der deutschen Musikbranche verliehen werden (ML AB 2: 7). Weitere Informationen bleiben ähnlich wie in Band 1 vor allem auf den Alltag begrenzt: so finden sich im zweiten Kursbuchband noch folgende landeskundlichen Inhalte: Verkehrsmittel im Alltag (ML KB 2: 16), in diesem Zusammenhang die Schwebebahn in Wuppertal (ML KB 2: 17), zur Übung der Wegbeschreibung eine Karte von Berlin (ML KB 2: 18), deutsche kulinarische Spezialitäten auf einer Speisekarte (ML KB 2: 31). Ausgehend von diesen Inhalten sollen die Lernenden selbständig weiterarbeiten und u.a. Preise verschiedener Lebensmittel im deutschsprachigen Raum recherchieren (ML AB 2: 37) oder eine kulinarische Spezialität aus den deutschsprachigen Ländern und ihre Zubereitung beschreiben (ML AB 2: 41).

$\mathrm{Zu}$ aktiver selbständiger Erarbeitung landeskundlicher Inhalte werden die Lernenden unter anderen in Recherche-Aufgaben angeregt. So soll z. B. das Schulnotensystem in der Schweiz und in Österreich ermittelt werden (ML AB 1: 35). Solche Aufgaben befinden sich zum Abschluss einzelner Lektionen sowohl im Lehr-als auch im Arbeitsbuch. Das Arbeitsbuch zu Band 1 bietet noch zwei weitere projektähnliche Aufgaben mit landeskundlichem Fokus: eine Recherche 
und anschließend das Erstellen eines Posters mit beliebten deutschen Vornamen für Mädchen und Jungen (ML AB 1: 7) und eine Recherche zu Möbeln: »Richte dein Zimmer ein. Wähle Möbel für dein Traumzimmer auf den deutschen WWW-Seiten aus.« (ML AB 1: 57).

Weiteren landeskundlichen Inhalten wird Platz auf den sich im Anhang sowohl des Lehrbuches als auch Arbeitsbuches befindenden `Kulturlink«-Seiten eingeräumt. In Band 1 (ML KB 1: 68-72, ML AB 1: 74-77) gehören zu den dort besprochenen Themen: Feste feiern; Zahlen, bitte; Liebesbeweise; Deutschlands schönstes Stadion; Mein Smartphone. Zu den Themen in Band 2 (ML KB 2: 7074, ML AB 2: 74-77) zählen: Projektwoche; Geburtstagswünsche; Kollege Hund; Etwas typisch Deutsches; Ein Sonntag in Deutschland.

Beide Kursbuchbände sind mit farbigen Fotos (nur vereinzelt gibt es auch gezeichnete Illustrationen) unterlegt. Die Fotos sind qualitativ hochwertig, sie wirken aber nur begrenzt authentisch. Wie man den Quellenangaben entnehmen kann (ML KB 1:80, ML KB 2:80), stammen sie - wie heutzutage die Fotos in nahezu allen globalen oder regionalen Lehrwerken - größtenteils aus freien Bilddatenbanken, was zur Folge hat, dass sie letztlich beliebig austauschbar wirken, d.h. sich auch in anderen Lernkontexten oder für das Lernen anderer Fremdsprache zum jeweiligen Thema eignen würden. In Mit links! sind die zahlreichen Portraitfotos von vor allem (gleichaltrigen) Jugendlichen besonders auffallend. Dies gilt jeweils für die Startseite der einzelnen Einheiten, bei denen zumindest noch begrenzt ein thematischer Zusammenhang von Foto und Lektionsinhalt erkannt werden kann. Als Beispiel kann hier Einheit 3 dienen. Beim Thema `Schule`gibt es ein Foto mit zahlreichen Büchern auf dem Tisch, darüber anscheinend lernende Jugendliche, dabei die Lehrerin (bzw. eine erwachsene Frau), die auf etwas im Buch zeigt (ML KB 1: 32). Auch die innerhalb der Lektionen präsentierten Fotos bilden fast ausschließlich Personen ab (z. B. ML KB 1: 8 Ü1 A, 1: 10 Ü1, 1: 20 Ü1, 1: 32 Ü2b), oft dienen sie der Illustration von kurzen Lesetexten (z.B. ML KB 1: 9 Ü1, 1: 11 Ü1, 1: 19 Ü1). Die Fotos von Gleichaltrigen sollen vermutlich Identifikationsmöglichkeiten bieten, sie sagen jedoch insgesamt wenig über den deutschsprachigen Raum aus.

\subsubsection{Landeskundeansätze und Prinzipien in Mit links!}

Vielfalt der deutschen Kultur- und Sprachlandschaft

Die kulturelle und sprachliche Vielfalt des deutschsprachigen Raums wird am Anfang des Kursbuches thematisiert. Das Autorenteam von Mit links! wählt einen Zugang zur deutschen Sprache über landeskundliche Informationen (vgl. Abbildung 22). So beginnt Lektion 1 mit Begrüßungen (ML KB 1:6), wobei nicht nur deutschsprachige bzw. DACH-typische Begrüßungs- und Abschiedsflos- 
keln, sondern auch ein multilingualer Einstieg mit Begrüßungsfloskeln aus verschiedenen Ländern, in denen germanische Sprachen gesprochen werden, gewählt wird.

Diese Visualisierung soll den Lernenden helfen, zu erkennen, dass Deutsch in mehreren Ländern gesprochen wird (DACHL), aber auch Ähnlichkeit mit einer Reihe anderer, in Europa gesprochener Sprachen hat, u. a. mit Englisch, der in den meisten Fällen ersten, und bereits seit mehreren Jahren gelernten Fremdsprache der Lernenden. Die neu zu lernende Fremdsprache Deutsch wird so, auch visuell in einen europäischen Kontext gesetzt.

Auf den Umschlagrückseiten im Kursbuch finden sich farbige Landkarten von Österreich, der Schweiz und Liechtenstein (Vorderumschlag) und Deutschland (Rückumschlag). In den einzelnen Einheiten finden sich nur wenige Aufgaben, die die kulturelle und sprachliche Vielfalt des deutschsprachigen Raums aufgreifen.

\section{Bezug zur Lebenswelt der Zielgruppe}

Bereits die die erste Lektion abschließende projektähnliche Aufgabe (১Projektlinkı): "Sammelt zu Hause originale Verpackungen von deutschen, österreichischen oder schweizerischen Produkten und macht Plakate in Gruppen " (ML KB 1: 6: Ü6) ermöglicht einen persönlichen Bezug aus der eigenen Lebensumgebung zu den DACH-Ländern herzustellen. Auch im weiteren Verlauf der ersten Einheit wird unter der Teilüberschrift `Meine Kursgruppe` indirekt ein europäischer Bezug hergestellt. Es werden z.B. Deutschlerner aus verschiedenen europäischen Ländern (Frankreich, Tschechien, Spanien und Ungarn) präsentiert (ML KB 1: 9 Ü1), so dass deutlich wird, dass Deutsch in verschiedenen Ländern gelernt wird. Dabei wird auch die Möglichkeit, Deutsch in Deutschland zu lernen bzw. dort Deutschkurse zu besuchen, erwähnt (»Jetzt bin ich in Deutschland und besuche den Anfängerkurs.«; »Ich bin jetzt in München. Ich mache hier einen Deutschkurs. «). Diesen Abschnitt abschließend wird ein Bezug zu den Lernenden selbst hergestellt (ML KB 1: 9, ÜB 5a und 5b), indem sie einen kurzen vergleichbaren Text über sich selbst schreiben sollen.

Obwohl die polnische Ausgangskultur im Lehrwerk nicht explizit aufgenommen wird, wird sie in den Aufgabenstellungen immer wieder berücksichtigt. In beiden Bänden gibt es Stellen, an denen der Bezug zur eigenen Situation hergestellt wird: die Lernenden sollen u. a. ein Poster oder eine multimediale Präsentation über beliebte Haustiere (ML KB 1:36) vorbereiten, über die Höhe ihres Taschengeldes sprechen (ML KB 1: 16), Informationen über den Schülern bekannte beliebte Musikbands recherchieren und vorstellen (ML KB 2: 7) oder über Essgewohnheiten in der Klasse sprechen (ML KB 2:28).

Die Lehrwerkautoren haben versucht, einen Bezug zur Lebenswelt der polnischen Schüler herzustellen, nicht nur durch die Wahl von zielgruppenge- 


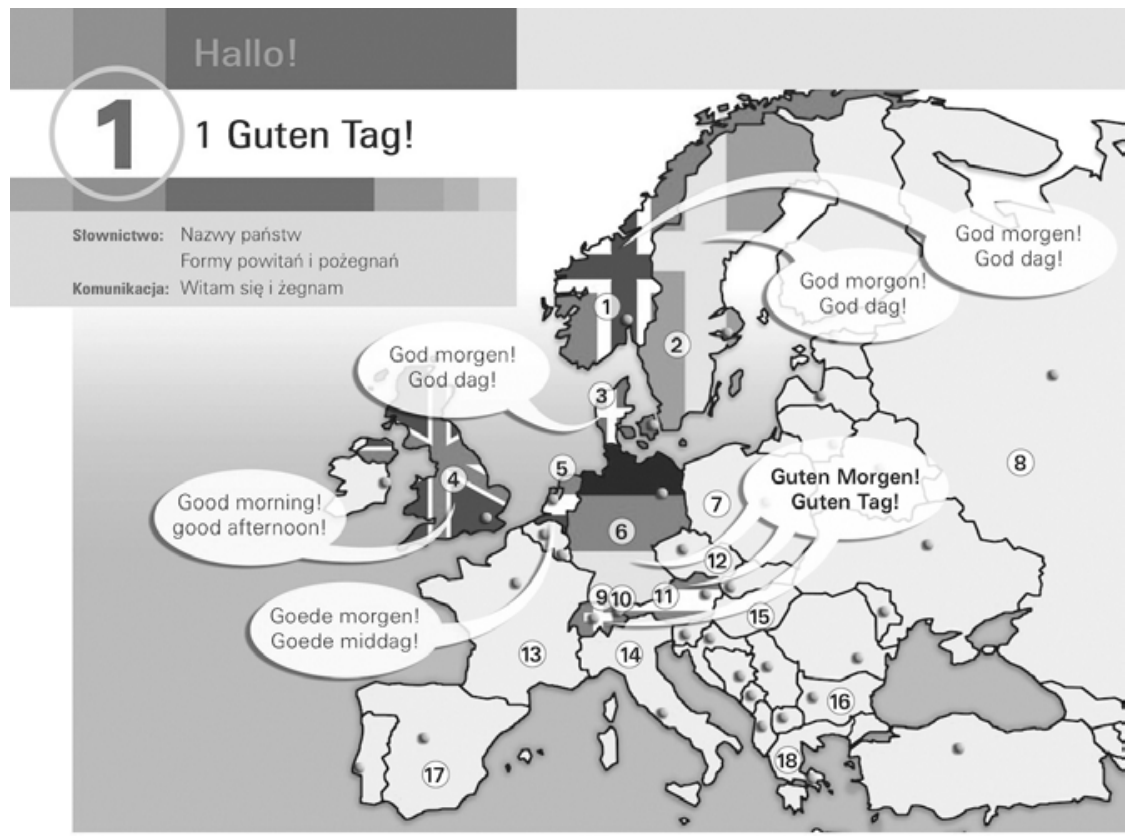

$1 \mathrm{~A}$ Finde auf der Landkarte Ländernamen. Znajiż na mapie ponizsze państwa.

\begin{tabular}{|ll|}
\hline Bulgarien & Liechtenstein \\
Dănemark & Österreich \\
Deutschland & Polen \\
England & Russland \\
Frankreich & Schweden \\
Griechenland & die Schweiz \\
Italien & Spanien \\
Norwegen & Tschechien \\
die Niederlande & Ungarn \\
\hline
\end{tabular}

Przed niektórymi narwami państw w języku angielskim pojawia się przedimek the. W języku niemieckim pojawia się rodzajnik die. Zarówno w języku angielskim, jak i w języku niemieckim nie można tych stówek pominąć.

1 B (i) Höre zu und wiederhole.

Sluchaj i powtarzaj zgodnie z nagraniem.

\section{Schreibe vier Länder ins Heft, in welchen man} Deutsch spricht.

Zanotuj w zescycie narwy ceterech państw, w których mówi się w jęz)ku niemiedkim.

\section{3 (3) Höre zu. Wo grüßt man so?}

Sluchaj pozdrowień z różnych państw. Zgaduj z jakich.
4 (4) Höre die Begrüßungen und wiederhole sie. Postuchaj pozdrowień i pożegnań i powtarzaj zgodnie z nagraniem.

Guten Morgen! Auf Wiedersehen!

Guten Tag! Tschüs!

Guten Abend! Bis dann!

Gute Nacht!

Grüß Gott!

Hallo!

5 (1) Höre zu und ordne im Heft zu. Postuchaj nagrania i do kaźdego dialogu dopasu' odpowiedni rysunek. Rorwięzanie zapisz w zeszycie.
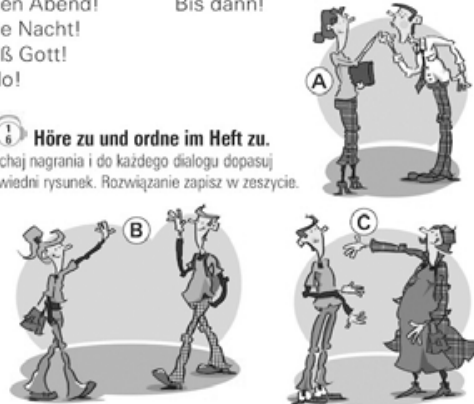

6 Projoktink Sammelt zu Hause originale Verpackungen von deutschen, österreichischen oder schweizerischen Produkten und macht Plakate in Gruppen.

Zbierzcie w domu oryginalne opakowania po niemieckich, szwajcarskich i austriackich produktach i wykorzystajcie je przy wykonywaniu plakatów w grupach na temat Niemiec, Austrii i Srwaicari.

Abbildung 22: Begrüßungsfloskeln in Europa (Mit links! KB 1: 6) 
rechten Themen (z.B. Schule; Meine Welt; Mein Wohnort), sondern auch durch den Einbezug von digitalen Medien, die fest zum alltäglichen Leben polnischer Jugendlicher gehören. Vor allem in Band 2 werden die Schüler dazu aufgefordert, Informationen selbständig im Internet zu recherchieren und die Ergebnisse mithilfe von multimedialen Präsentationen vorzustellen (ML KB 2: 17, ML KB 2: 41) (vgl. Abbildung 23).

\section{Stimulierung einer kulturreflexiven Haltung}

Die einzelnen Inhalte in Mit links! bieten viele Anlässe für internationale Bezüge, bei denen teils direkt zum Vergleich angeregt wird, meistens bleibt es jedoch den Lehrern überlassen, ob und inwieweit die angebotene Kontrastivität Eingang in das Unterrichtsgeschehen findet. In Lektion 3 wird beispielsweise implizit das deutsche Notensystem präsentiert (ML KB 1: 27 Ü2 und Ü3). Hier wird ein relevantes und sicher auch für die Zielgruppe interessantes landeskundliches Thema erwähnt (Notengebung in Deutschland). Dadurch, dass aber nur die versprachlichten Begriffe (sehr gut, gut usw.) genannt werden, kann es ohne die Lehrperson kaum zu einem Vergleich mit dem eigenen Notensystem kommen, und somit auch nicht zu einer Reflexion hierüber. Der nummerische Vergleich wird nicht geboten: sehr gut $=1 \neq 6$ (polnische Bestnote). Die Lektion abschließend sollen die Lernenden im Internet Informationen über die Schulnoten in Österreich und in der Schweiz suchen (ML AB 1: 35).

Gleiches gilt für die Abbildung eines Stundenplans als Einstieg in die Lektion 3 (ML KB 1:26), die wie eine authentische Abbildung eines Stundenplans in einer deutschen Schule (Sek. I) wirkt. Es handelt sich um implizit vermittelten Landeskunde-Inhalt, da nicht explizit verglichen bzw. zum Vergleich angeregt wird (ML KB 1: 26 Ü3b) und somit auch nur mit Hilfe der Lehrperson reflektiert werden kann. Lernende sollen in der anschließenden Übung sagen, welche Fächer sie selbst beispielsweise am Montag oder wie viele Unterrichtsstunden sie am Dienstag haben.

Gelungene Beispiele für eine Übertragung auf die eigene Lebenswirklichkeit der Lernenden, die zum Vergleich oder zur Reflexion einladen, stellen die `Kulturlink`-Seiten dar. In der Lektion »Feste feiern« erhalten die Lernenden Informationen über drei Feste in Deutschland: Weihnachten, Silvester und Karneval. Die anschließende Aufgabe regt zu einem Vergleich mit dem eigenen Land an: "Wählt in Paaren ein Fest aus Aufgabe 1 und stellt es in der Klasse vor. Berichtet: Wie feiern wir dieses Fest in Polen? Verwendet den Wortschatz unten.«(ML KB 1: 70) (vgl. Abbildung 24).

Auch weitere Lektionen enthalten Aufgaben, die es ermöglichen, Bezüge zu der Welt der Lerner zu schaffen und zu einem ggf. auch reflektierten Vergleich anzuregen: »Macht in Gruppen in euren Heften ein Ranking der in eurer Familie am häufigsten genutzten Zahlungsmöglichkeiten. Präsentiert eure Ergebnisse in 


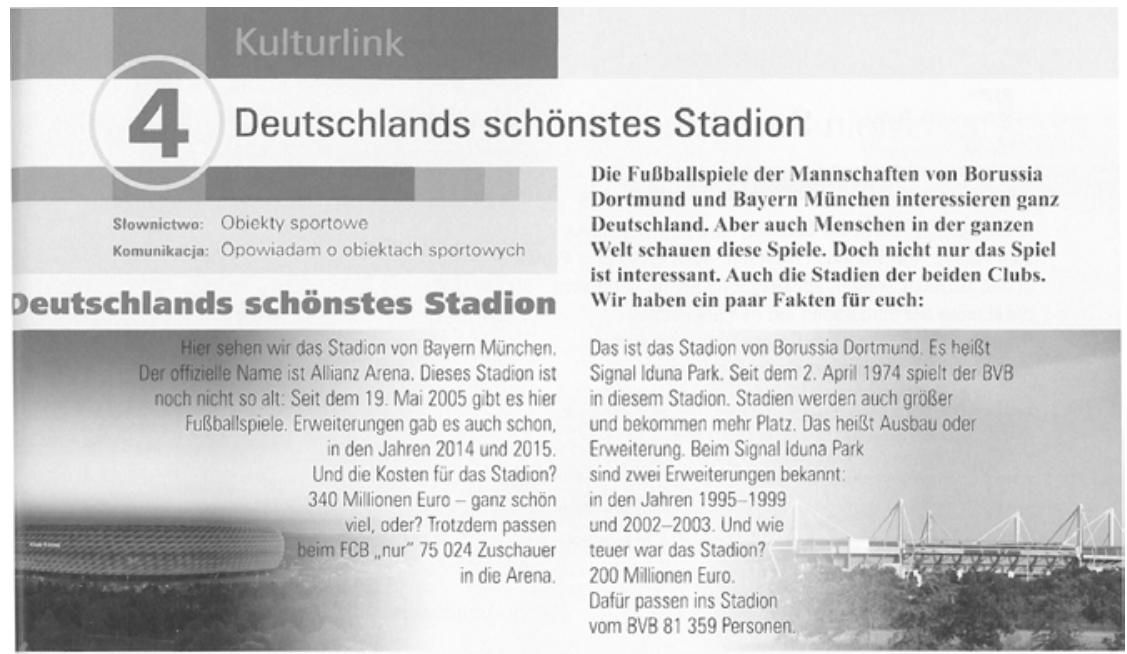

Eins haben aber beide Stadien gemeinsam - die Spielfläche ist $105 \mathrm{~m}$ x $68 \mathrm{~m}$ groß. Nur eine Frage ist noch offen: Welches Stadion findet ihr schöner?

$1 \mathrm{~A}$ Lies den Text und finde die passenden Satzteile. Schreibe die Lösung ins Heft.

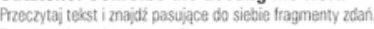
Rocwiązanie zapisz w zesrycie

1 Die beiden Stadien sind nicht

2 Die zwei Stadien sind

3 Die Spielfläche der zwei Stadien ist

A gleich groß.

B gleich alt.

C nicht gleich teuer.

1B Lies den Text noch einmal und notiere im Heft die fehlenden Informationen wie in der Tabelle angegeben.

Precrytaj jesccre raz tekst i zrób notatki w zeszycie zgodnie z informaciami podanymi w tabeli.

\begin{tabular}{l|l|l} 
Kategorie & $\begin{array}{c}\text { Borussia } \\
\text { Dortmund } \\
\text { BVB }\end{array}$ & $\begin{array}{c}\text { Bayern } \\
\text { München } \\
\text { FCB }\end{array}$ \\
Offizieller Name & & \\
\hline Alter & & \\
\hline Erweiterungen & & \\
\hline Kosten & & \\
\hline Maximale Zuschauer & & \\
\hline Spielfläche & &
\end{tabular}

2 Sprecht in der Klasse darüber, welches Stadion in Polen am schönsten ist.

Porozmawiajcie w klasie o tym, który stadion w Polsce jest ną̧adriejszy.

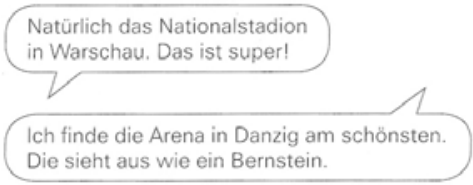

3 Wobink Findet im Internet Informationen über ein Stadion in Polen zu den angegebenen Punkten und bereitet in Paaren eine multimediale Präsentation mit Foto vor.

Pracuigc w parach, zgodnie z podanymi punktami wyszukajcia

w internecie informaci na temat znanego stadionu w Polsce, i w wniki predstawcie w formie prezentaci multimedialnej ze zojpciem.

- Offizieller Name

- Alter

- Kosten

- Maximale Zuschauer

- Spielfläche

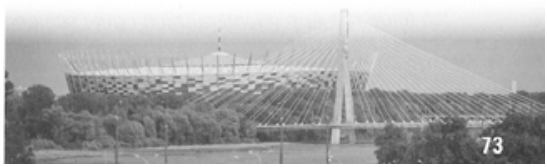

Abbildung 23: Bezug zur Lebenswelt der Jugendlichen am Beispiel des Themas Sport: Eine Recherche-Aufgabe zu Stadien in Polen (Mit links! KB 1: 73) 

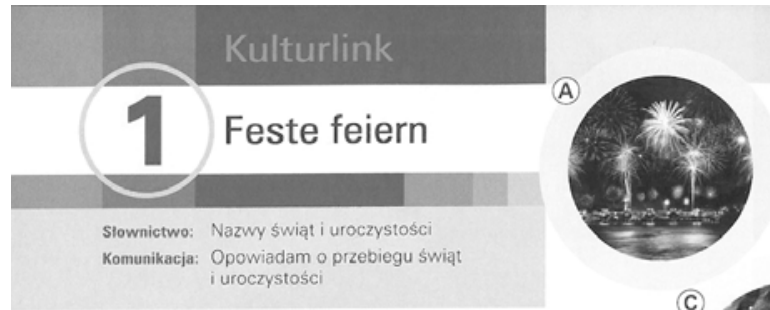

(B)

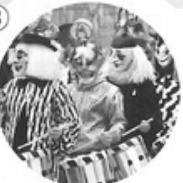

(1)

Weihnachten feiern wir in Deutschland, Österreich und der Schweiz meistens zu Hause. Mit der ganzen Familie. An Heiligabend, das ist der 24. Dezember, bekommen alle Geschenke. Kinder singen Weihnachtslieder und am Weihnachtsbaum brennen die Kerzen.

Am ersten und zweiten Weihnachtsfeiertag essen wir sehr viel. Oft zu viel. Aber alle mögen Weihnachten.

(2)

(C)

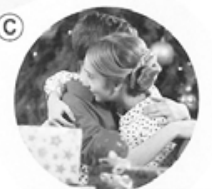

(D)

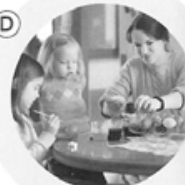

An Silvester feiern die meisten Menschen in Berlin, Zürich und Wien mit der Familie oder mit Freunden. Was ist besonders?

Viele machen Vorsätze, das sind Pläne fürs neue Jahr. Zum Beispiel: „Ich mache mehr Sport." Oder: "Ich esse weniger und gesund."

Am Himmel gibt es ein Feuerwerk, um 24 Uhr stoßen alle an und sagen: "Prost Neujahr." Viele Menschen schenken ein kleines Schwein, als Talisman oder zum Essen aus Marzipan. Sie hoffen, das rosa Schweinchen bringt Glück.

Karneval ist in Deutschland, Österreich und der Schweiz eine regionale Sache. In manchen Regionen und Städten ist das ein wichtiger Feiertag, in anderen nicht.

Die Traditionen sind überall anders, die Namen auch: In Basel, Mainz oder in Schaan sagen wir Fasnacht, in Bayem und Österreich Fasching und in Köln, Düsseldorf oder Bonn Karneval. Die Traditionen sind aber ähnlich. Alle feiern, gehen auf eine Parade, tragen Masken oder ein verrücktes Kostüm und sind fröhlich.

1 Lies die Texte und sage: Welches Foto passt zu welchem Text? Ein Foto passt zu keinem Text. Przeczytaj teksty i powiedz, które zdjęcie pasuje do którego tekstu Jedno adjęcie nie pasuje do zadnego tekstu.

2A Schreibt in Paaren in eure Hefte je vier Fragen zu jedem Text.

W zeszytach zapiszcie w parach po cztery pytania do kaźdego tekstu.

Wo feiern die Deutschen, Österreicher und Schweizer Weihnachten?

Mitwem ...

Wann ...

Was...

Wie...

...

\section{B (3) Stellt die Fragen in der Klasse.}

Die anderen Schüler beantworten die Fragen.

Zadajcie pytania na forum klasy. Pozostali uczniowie odpowiadaia na pytania.

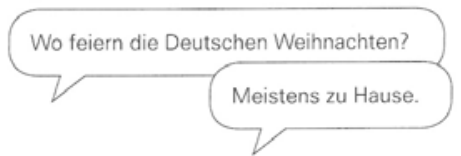

3 91) Sagt: Welche der drei Feste aus Aufgabe 1 gibt es auch in Polen? Welche Feste feiert ihr? Powiedzcie, które z trzech swiąt $z$ zadania 1. są równiez w Polscee. Które święta obchodricie?

In Polen gibt es auch...

Wir feiern immer...

Wir feiern ... nicht.

4 (3) Wählt in Paaren ein Fest aus Aufgabe 1 aus und stellt es in der Klasse vor. Berichtet: Wie feiern wir dieses Fest in Polen? Verwendet den Wortschatz unten.

W parach wybierzcie jedno świeto z zadania 1, i przedistawoie je klasie. Opowiedzcie, jak obchodzimy to swięto w Polsce. Wykorzystajcie poniższe slownictwo.

(nicht) arbeiten - (ganz) lange schlafen * (zu) viel essen * (nicht) in die Schule gehen * stundenlang einen Spaziergang machen * (nicht) in die Schule gehen - mit der (ganzen) Familie feiern $\boldsymbol{*}$ (alle) Freunde treffen • (tolle) Geschenke machen $\cdot$ nichts Besonderes machen * (endlich einmal) relaxen * essen / trinken

An Weihnachten essen wir viel. Wir ... immer ...

An Silvester feiern wir mit der Familie. Wir ... oft ...

Abbildung 24: Stimulierung einer kulturreflexiven Haltung am Beispiel des Themas Feste (Mit links! KB 1: 70) 
der Klasse.« (ML KB 1: 71) oder »Findet im Internet Informationen über ein Stadion in Polen zu den angegebenen Punkten und bereitet in Paaren eine multimediale Präsentation mit Foto vor.« (ML KB 1: 73).

Auch Band 2 enthält auf den `Kulturlink«-Seiten kurze Informationstexte mit landeskundlichem Fokus samt Anregungen für weitere Beschäftigung mit dem jeweiligen Thema. Es werden die Projektwoche, Geburtstagswünsche und das Geburtstagslied »Viel Glück und viel Segen«, ein Hund im Büro und anschließend die Idee eines Klassenhundes, Abschiedsgeschenk für Austauschschüler sowie ein verkaufsoffener Sonntag (ML KB 2: 72) thematisiert. Durch die hierzu angeregte Diskussion über mögliche Vor- und Nachteile, kann kulturreflexives Lernen initiiert werden.

Während die Übungen und Aufgaben in den Kursbüchern überwiegend zur Diskussion in der Zielsprache anregen, liegt der Schwerpunkt in den darauf abgestimmten Übungen im Arbeitsbuch größtenteils auf Wortschatzarbeit.

\section{Landeskunde erfahren}

Bis auf eine Aufgabe in Band 1 enthält keiner der beiden Bände von Mit links! Aufgaben, in denen die Lernenden die Möglichkeit hätten, die deutschsprachigen Länder direkt zu erleben. Die einzige oben genannte projektartige Aufgabe (ML KB 1:6) regt die Schüler in der allerersten Lektion dazu an, „zu Hause originale Verpackungen von deutschen, österreichischen oder schweizerischen Produkten zu sammeln« und dazu Plakate zu gestalten.

\section{Fazit}

Mit links! ist zweifelsohne ein kommunikativ orientiertes Lehrwerk. Die behandelten Themen sind adressatengerecht ausgewählt und entsprechen den Interessen der jungen Lerner. Die landeskundlichen Informationen, die in beiden Bänden geboten werden, werden überwiegend implizit vermittelt. Das Lehrwerk enthält Anregungen zu eigenständigen Recherchen sowie zum kulturreflexiven und multilingualen Vergleich. Die sich im Anhang befindlichen `Kulturlink`-Seiten mit explizitem Landeskundefokus haben einen fakultativen Charakter. Die dort präsentierten Inhalte und die dazugehörigen Übungen sowie projektorientierten Aufgaben sind durchdacht, anregend und sollten deshalb in die Lehrwerkeinheiten integriert werden. Ihre Position im Anhang verleiht ihnen einen Sonderstatus und verbannt sie folglich zu Unrecht aus dem »Pflichtprogramm« für den Unterricht. 


\subsubsection{Kompass Team}

Kompass Team stellt eine überarbeitete Ausgabe des in den späten 2000er Jahren konzipierten Lehrwerkes Kompass dar, das von demselben Autorenteam zunächst zu Kompass neu und schließlich 2017 zu Kompass Team überarbeitet wurde. Das kommunikativ orientierte Lehrwerk kann entweder in Klassen eingesetzt werden, die erst beginnen, Deutsch zu lernen (Band 1 in Klasse 7 und Band 2 in Klasse 8), oder in Klassen, in denen Deutsch fortgesetzt wird (dann entsprechend Band 2 und Band 3). Der besseren Vergleichbarkeit halber werden in der folgenden Analyse Kompass Team Band 1 und Band 2 berücksichtigt, die nach GER zum Sprachniveau A1+ führen.

$\mathrm{Zu}$ den Lehrwerkskomponenten gehören pro Klassenstufe ein Kursbuch mit integrierten Audio-CDs und ein Arbeitsbuch. Den Lehrkräften steht zusätzlich ein online Lehrwerkservice zur Verfügung, der u.a. Zugang zu Modelltests und Kopiervorlagen bietet.

Das Kursbuch besteht pro Band aus 5 Einheiten, die in 5 Lektionen unterteilt sind. Für die Bearbeitung jeder Lektion sind jeweils 2 Unterrichtsstunden vorgesehen. $\mathrm{Zu}$ den Themen, die in diesen zwei Lernjahren angesprochen werden, gehören:

Band 1: Kontakte; Familie; Alltag, Freizeit; Schule; Essen,

Band 2: Der Kalender; Interessen, Pflichten, Träume; Wohnen; Das Leben schreibt Geschichten; Reisen.

In den Einheiten 1, 3 und 5 (jeweils in Band 1 und 2) befinden sich zwei zusätzliche >Interregio-Seiten, die der Förderung der interkulturellen Kompetenz dienen. Am Ende des Kursbuchs (KT KB 1: 128, KT KB 2:133) gibt es jeweils fünf Anregungen für Projekte, die auf die einzelnen Einheiten thematisch abgestimmt sind. Dem folgen Grammatikerläuterungen (KT KB 1: 129-133, KT KB 2: 134-136), eine tabellarische Grammatikübersicht (KT KB 1: 134-137, KT KB 2: 137-142, in Band 2 zusätzlich noch eine Tabelle der unregelmäßigen Verben KT KB 2: 143) und ein alphabetisch geordnetes deutsch-polnisches Wörterbuch (KT KB 1: 138-142, KT KB 2: 144-150). Die Redemittel mit ihrer Übersetzung ins Polnische sind den Einheiten nach gegliedert (KT KB 1: 143, KT KB 2: 150151). Die letzte Seite in jedem der zwei Bände enthält einen Lösungsschlüssel zu den Übungen auf den Kompass-Training-Seiten sowie eine Sammlung von Lernstrategien in Form von kurzen Reimen, die über die einzelnen Lektionen verteilt waren (KT KB 1: 144, KT KB 2: 152).

Das 71-seitige Arbeitsbuch Kompass Team Band 1 und das 84-seitige Arbeitsbuch zu Band 2 bieten vertiefende Übungen zu Wortschatz und Grammatik. Jede Einheit im Arbeitsbuch schließt mit einer `Kompass-Training` Seite ab, 
die eigentlich nichts anderes als eine Lernwortschatzseite ist und eine Sammlung deutscher Vokabeln mit Platz für deren Übersetzung bietet.

\subsubsection{Stellung und Art der Vermittlung von Landeskunde in Kompass Team}

Das Lehrwerk Kompass Team vermittelt kultur- und landeskundliche Informationen weitgehend implizit. Sie sind in die einzelnen Lektionen integriert und bleiben größtenteils auf Faktenwissen beschränkt. Implizite Landeskunde findet sich z.B. in der Lektion >Feiertage (KT KB 2:16), in der Kardinalzahlen und Datumsangaben mithilfe der Termine von Fest- und Feiertagen in den DACHLändern vermittelt werden. An der Stelle erfahren die Schüler nichts über die einzelnen Feiertage, deren Verlauf, Bräuche oder Herkunft. Ähnlich wird bei dem Thema >Haus der Jugend in Band 2 (KT KB 2: 40) vorgegangen, wo in einem Lesetext die Jugendfreizeiteinrichtung mit ihren einzelnen Räumen und den Möglichkeiten der Freizeitbeschäftigung vorgestellt wird, sich aber keine Anregungen für eine vertiefende Auseinandersetzung mit dem (landeskundlich orientieren) Inhalt finden. Der Text bietet zwar einen Ausschnitt kultureller Wirklichkeit, der Schwerpunkt der Beschäftigung mit dem Text liegt aber auf der Vermittlung eines grammatischen Phänomens (Modalverb können).

Auch mittels name dropping erscheinen landeskundliche Inhalte implizit und so werden in Lesetexten und den anschließenden Übungen beispielsweise Städtenamen aus dem deutschsprachigen Raum (u.a. Bonn, Linz, Hartnau, Frankfurt, Wien, Salzburg), Fußballvereine (Bayern München, Werder Bremen), Währung, Namen von Fernsehsendern (DSF und Sat 1) oder ausgewählte Schultypen (Gymnasium, Realschule) erwähnt.

Explizite landeskundliche Informationen haben ihren festen Platz im Lehrwerk; sie werden auf den vorgeschalteten Seiten in Band 1 >Länder in Europa (KT KB 1:6,7) und in Band 2 >DACH-Länder -Seiten (KT KB 2:6, 7) präsentiert. Den expliziten landeskundlichen Informationen sind auch die >Interregio<Seiten (KT KB 1: 28-29, 78-79, 124-125, KT KB 2: 32-33, 82-83, 130-131) gewidmet.

Die Vermittlung der landeskundlichen Kenntnisse wird durch Fotografien und Illustrationen unterstützt. Sie zeigen, worüber Informationstexte berichten, z. B. die Fotografien architektonischer Besonderheiten wie das Paul Klee Zentrum in Bern, das Sony Center in Berlin oder das Hundertwasserhaus in Wien (KT KB 2: 82-83). Die Fotos dienen darüber hinaus dazu, Gegenstände und bestimmte Sachverhalte zu beschreiben, wie z. B. die Fotos von Verkehrsmitteln in Deutschland (KT KB 2:116), hierzu auch eine Abbildung des Liniennetzes der S-Bahn und U-Bahn in Berlin (KT KB 2: 118-119). Es gibt auch Fotos, deren Auswahl eher beliebig scheint, der Zusammenhang mit den präsentierten Inhalten bleibt offen, wie z.B. die Illustration, die ein junges Mädchen mit einem 
Notizbuch in der Hand darstellt (KT KB 2: 97, für weitere Beispiele auch KT KB 1: 85, KT KB 2: 28). Die Funktion dieser Fotos dürfte auf die Auflockerung der Lehrwerkseiten beschränkt sein.

\subsubsection{Landeskundeansätze und Prinzipien in Kompass Team}

\section{Vielfalt der deutschen Kultur- und Sprachlandschaft}

In Band 1 von Kompass Team werden auf zwei vorgeschalteten Seiten mit dem Titel >Länder in Europa in sechs unterschiedlichen Übungen die Namen der europäischen Länder, Begrüßungen und Flaggen der einzelnen Länder präsentiert. In weiteren drei aufeinander folgenden Übungen wird nach den deutschsprachigen Ländern und ihren Hauptstädten gefragt (KT KB 1: 7 Ü4), wobei deren Flaggen und Länderkennzeichen präsentiert werden. Die Lernenden sollen auch die Fragen »Wie viele Menschen sprechen Deutsch?« (KT KB 1: 7 Ü5) und »In welchen Ländern zahlt man in Euro?« (KT KB 1: 7 Ü6) beantworten. Ähnlich sind die in Band 2 vorgeschalteten Seiten mit Informationen zu den DACH-Ländern konzipiert. Auch hier sollen die Lernenden Aufgaben lösen, die Faktenwissen abverlangen, wie z. B. in Übung 2 (KT KB 2: 6), wo die Lerner Fluss-, Gebirgs- und Städtenamen den DACH-Ländern zuordnen sollen. Sie sind im Kursbuch wie folgt aufgelistet: "Vaduz, Donau, Elbe, Wien, Harz, Berlin, Bern, Alpen, Aare, Zürich, München, Salzburg und Rhein«. In Aufgabe 3 sollen die Lernenden in fünf vorgegebenen Sätzen aus den vorgegebenen Informationen über Deutschland (Zahl der Bundesländer, die geographische Lage der einzelnen Bundesländer) die richtigen unterstreichen (z. B. »Deutschland besteht aus 16/12 Bundesländern.«).

Die >Interregio‘-Seiten fokussieren landeskundliche Inhalte, die die Vielfalt der deutschen Kultur- und Sprachlandschaft zeigen. In Band 1 wird vor allem die Plurilingualität der deutschen Sprache in Gruß- und Abschiedsformeln aus den DACH-Ländern (KT KB 1: 28-29) und in landesspezifischen Bezeichnungen bestimmter Lebensmittel, wie z. B. Brötchen, Bonbon oder Tomate (KT KB 1: 124-125) erkennbar. Band 2 bietet den Lernern in drei kurzen Lesetexten Informationen zum Nikolaus in den DACH-Ländern (KT KB 2: 32-33), Einblicke in die Architektur von Paul Klee, Helmut Jahn und Friedensreich Hundertwasser (KT KB 2: 82-83) sowie einen Vergleich der Dienstleistung eines Citybike-Verleihs in der Schweiz, in Österreich und in Deutschland (KT KB 2: 130-131). Den Lernenden wird hier also ein kleiner Einblick in die sprachliche und kulturelle Diversität des deutschsprachigen Kulturraums gewährt.

Durch die separate Präsentation der >Interregio〈-Seiten wirken sie eher wie ein fakultatives Angebot, da die Lehrkräfte auf die Bearbeitung problemlos, d.h. 
ohne Konsequenzen für den weiteren, im Lehrbuch angelegten Lernprozess, verzichten können.

Ein Beispiel für die kreative Vermittlung landeskundlicher Inhalte stellt die Anregung für das erste `Projekt` dar (KT KB 1: 128). Die Lernenden können in dieser projektorientierten Aufgabe selber aktiv werden und in Kleingruppen Informationen $\mathrm{zu}$ einem, von ihnen ausgewählten, deutschsprachigen Land recherchieren und anschließend in Form einer Collage in der Klasse präsentieren. Sie werden dazu angeregt, Illustrationen, Fotos, Texte, Verpackungen und andere Materialien zu gebrauchen.

\section{Bezug zur Lebenswelt der Zielgruppe}

Im Allgemeinen orientieren sich die landeskundlichen Inhalte an Themen, die die Jugendlichen betreffen, u. a. Familie, Freizeit, Schule, Feiern, Essen, Wohnen, Reisen. Die Lernenden erfahren u. a. etwas darüber, was für Interessen (eine Statistik zum Thema "Die Deutschen im Sammelfieber" KT KB 2: 36), Pflichten (KT KB 2: 46) oder Berufswünsche (KT KB 2:55) ihre Altersgenossen in den DACH-Ländern haben oder welche Essgewohnheiten sie haben (»Frühstück in Deutschland« KT KB 1: 108, 109; »Deutsch essen international« KT KB 1: 104). Sie lernen aber auch touristische Sehenswürdigkeiten der einzelnen Städte kennen (»Herzliche Grüße aus Wien!« KT KB 2: 126, 127). Viele der anschließenden Aufgaben erlauben es den Schülern, Bezüge zur eigenen Lebenswelt herzustellen. Sozialformen in den Aufgaben variieren von der Einzelarbeit (»Was nimmst du in die Schule mit?« KT KB 1: 111, „Wie sieht dein Weg zur Schule und von der Schule nach Hause aus? Notiere im Heft und beschreibe ihn.» KT KB 2:111), über Partnerarbeit (»Sprecht über eure Interessen.« KT KB 1:49) bis hin zu Gruppenarbeit (»Erstellt eine Präsentation über euren Lieblingslehrer« KT KB 1: 97, »Was macht ihr in eurer Freizeit?« KT KB 2: 39).

An den Stellen im Lehrwerk, wo Landeskunde explizit dargestellt wird, werden die Lernenden oft dazu angeregt, präsentierte landeskundliche Inhalte mit ihrer eigenen Situation zu vergleichen, wie bei der Präsentation der Feste (»Welches Fest magst du am liebsten?«KT KB 2: 25), der Essgewohnheiten der deutschen Jugendlichen (»Was isst man in Polen in der Schule?« KT KB 1:111) oder im Zusammenhang mit einem tabellarischen Vergleich der Schulnoten in DACH-Ländern (»Wie sieht das Schulnotensystem in Polen aus?« KT KB 1: 83).

\section{Stimulierung einer kulturreflexiven Haltung}

In beiden Bänden überwiegen projektorientierte Aufgaben zu Alltagsthemen, für deren Lösung die Lernenden keine kultur- und landeskundlichen (Hintergrund-)Informationen brauchen und/oder berücksichtigen sollen. Vereinzelt gibt es in Kompass Team größere, die Lernenden stärker aktivierende landeskundlich orientierte Projekte, in denen sie dazu angeregt werden, die ver- 
meintlich eigene und fremde Kultur zu vergleichen. In aller Regel handelt es sich hierbei darum, Faktenwissen zu vergleichen, wie beispielsweise in den folgenden Aufgaben »Erstellt ein Plakat zu Polen (Lage, Nachbarländer, die größten Städte, Flüsse, Gebirge, ...) ( (KT KB 2: 7), »Erstellt ein Plakat mit den Ferienterminen in Polen und in einem der deutschsprachigen Länder« (KT KB 2: 19).

Lediglich einige >Interregio<-Seiten bieten Aufgaben, in denen die Lernenden aufgefordert werden, unterschiedliche kulturelle Phänomene zu reflektieren. Beispielsweise beim Thema Stundenplan in einer schweizerischen Realschule (KT KB 1: 79) sollen nicht nur Bezüge zur eigenen Situation hergestellt werden (»Um wie viel Uhr ist bei euch Schulschluss?«), sondern die Lernenden sollen auch darüber diskutieren, ob ihnen die Idee einer langen Mittagspause und weiterer Unterrichtsstunden am Nachmittag gefällt. Auch beim Thema Nikolaus (KT KB 2: 33) wird auf die kulturellen Eigenheiten eingegangen, mit der anschließenden Aufgabe, über Gemeinsamkeiten zwischen den DACH-Nikolausmännern und dem polnischen Nikolaus zu reflektieren.

Die kontrastive Kulturvermittlung ist auf die Ausgangs- und Zielkultur(en) beschränkt. Die Bezüge zu anderen als der polnischen und deutschen Kultur (selten anderer deutschsprachigen Kulturen) werden in dem Lehrwerk kaum hergestellt. Sie gehen über Begrüßungsformeln (KT KB 1:6) und Herkunft einzelner Speisen (»Pizza und Spaghetti kommen aus ..., Gyros und Tsatsiki kommen aus ... K KT KB 1:104) kaum hinaus.

\section{Landeskunde erfahren}

Kompass Team enthält nur eine Anregung zu einem Projekt, das die Lernenden die deutsche Kultur und Sprache in der eigenen Umgebung direkt erfahren lässt. In dem ersten der im Anhang in Band 1 (KT KB 1:128) befindlichen, insgesamt fünf vorgeschlagenen Projekten werden die Lernenden mit realen Gegenständen, die sie in den Unterricht mitbringen sollen, wie Texte, Verpackungen, Aufkleber aus dem deutschsprachigen Raum konfrontiert.

\section{Fazit}

Kompass Team ist ein zweifelsohne kommunikativ orientiertes Lehrwerk, in dem die Alltagskultur im Vordergrund steht. Der Schwerpunkt der einzelnen Lektionen liegt auf für die Zielgruppe alltagsnahen Themen, die größtenteils ihren Interessen entsprechen dürften. Obwohl die Landeskunde an vielen Stellen in dem analysierten Lehrwerk nicht auf Anhieb erkennbar ist, da die landeskundlichen Informationen in beiden Bänden größtenteils implizit vermittelt werden, gewähren beide Lehrwerkbände den Lernenden einen Einblick in die kulturelle und sprachliche Vielfalt des deutschsprachigen Raums. Dabei ist vor allem ein faktischer Landeskundeansatz erkennbar. Die landeskundlichen Texte liefern Informationen zu Geografie und kulturellen (Feste) oder touristischen 
Besonderheiten (Sehenswürdigkeiten). Die Plurilingualität - wenn auch in begrenztem Umfang - wird ebenfalls thematisiert.

Gelegentlich wird in dem Lehrwerk ein kontrastiv-orientierter Ansatz sichtbar, wenn nach Unterschieden oder Gemeinsamkeiten zwischen der vermeintlich fremden und eigenen Kultur gefragt wird. Kulturreflexives Lernen wird jedoch in Kompass Team kaum initiiert.

Die landeskundlichen Informationen werden in beiden Bänden größtenteils implizit vermittelt. Die einzigen Lehrwerkseiten mit einem deutlichen Landeskundefokus - die ıInterregios-Seiten - haben einen fakultativen Charakter. Beides trägt dazu bei, dass die Entscheidung über die Thematisierung von Landeskunde im Unterricht den Lehrkräften überlassen bleibt.

\subsubsection{Meine Deutschtour}

Meine Deutschtour richtet sich an Schüler der Klassen 7 und 8. Für jede Klassenstufe sind ein Kursbuch und ein Arbeitsbuch vorgesehen. Den Lehrkräften steht zusätzlich ein Lehrerhandbuch zur Verfügung, das außer detaillierten methodisch-didaktischen Hinweisen eine Audio-CD und eine DVD mit Videoclips enthält.

Unterrichtstragend ist das Kursbuch, das pro Band sechs Einheiten enthält, die wiederum jeweils in acht Lektionen unterteilt sind. Den im GER genannten Inhalten auf A1-Niveau entsprechend werden in zwei Lernjahren folgende Themen aufgriffen:

Band 1: Familien in Deutschland; Deutsche Schule; Alltag in Deutschland; Essen in Deutschland; Sport und Rekorde nicht nur in Deutschland; Natur, Sonne und Sommerferien in Deutschland

Band 2: Gesundheit in Deutschland; Häuser in Deutschland; Feiertage und Feste in Deutschland; Stadt und Land in Deutschland; Modewelt in Deutschland; Lernen, Arbeit und Freizeit in Deutschland.

Auf der ersten Doppelseite jeder neuen Einheit ist ein großes, jeweils die Doppelseite ausfüllendes Foto abgebildet, das das Thema der Einheit einleitet. Auf der linken Seite der Doppelseite befindet sich eine Lernzielübersicht. In den acht einzelnen Lektionen der jeweiligen Einheit werden Wortschatz, Redemittel, grammatische Strukturen und landeskundliche Informationen vermittelt. An die einzelnen Einheiten schließen jeweils zwei Seiten mit zusätzlichem Material an: Dialogecke EXTRA und Textecke EXTRA, die der Förderung des Leseverstehens und der mündlichen Sprachkompetenz dienen. Jede Einheit endet mit einer vierseitigen Zusammenfassung. Jeweils zwei Seiten davon sind dem Ak- 
tivwörterbuch gewidmet, das wichtige Sätze, Floskeln und Redemittel aus der Einheit enthält. > Grammatik ist okay! ‘ präsentiert kurze Erläuterungen und eine Grammatikübersicht. Unter dem Abschnitt >Vor der Klassenarbeit! werden die Lernziele der Einheit (geordnet nach Wortschatz und Grammatik) noch einmal aufgelistet. Am Ende der Lehrbücher befinden sich:

- Projektseiten mit sechs thematisch auf die Einheit abgestimmten Vorschlägen zu landeskundlichen Projekten (MD KB 7: 140-145, MD KB 8: 138-143),

- Aussprachetraining (MD KB 7: 146-147, MD KB 8: 144-145),

- alphabetisch geordnetes deutsch-polnisches Wörterbuch (MD 7: 148-155, MD 8: 146-153) und

- grammatische Übersichten (MD KB 7: 156-163, MD KB 8: 154-166).

Die Struktur des Arbeitsbuches ist übersichtlich und auf das Lehrbuch abgestimmt. Im Arbeitsbuch können der Lernwortschatz und die grammatischen Strukturen wiederholt und trainiert werden.

\subsubsection{Stellung und Art der Vermittlung von Landeskunde in Meine Deutschtour}

Das Lehrwerk Meine Deutschtour legt großen Wert auf die Vermittlung von kultur- und landeskundlichen Informationen. In der Inhaltsübersicht sind explizit landeskundliche Inhalte aufgelistet. Die prominente Rolle, die Landeskunde in dem Lehrwerk Meine Deutschtour einnimmt, ist bereits zu Beginn des Lernprozesses sichtbar. Zahlreiche explizite landeskundliche Informationen aus dem deutschsprachigen Raum werden auf den vorgeschalteten Seiten als >Starter` präsentiert. Dort werden die Lernenden auf insgesamt 14 Seiten mit den Besonderheiten der DACH-Länder vertraut gemacht. Auch der sechzehnseitige 'Starter im zweiten Band enthält neben wiederholenden lexikalisch-grammatischen Übungen zu den Inhalten aus dem ersten Band fünf informative Seiten zu den DACH-Ländern, die mit einem DACH-Quiz mit zehn Fragen abgeschlossen werden (MD KB 8: 12-27).

In den einzelnen Lektionen in beiden Bänden ist die wichtige Rolle der Landeskunde zu erkennen. Das Lehrwerk zeigt eine gelungene Umsetzung des Ansatzes der integrativen Landeskunde. Die landeskundlichen Inhalte sind regulär in der jeweiligen Lektion integriert. Als ein Beispiel für die Integration von Spracharbeit und Vermittlung landeskundlicher Inhalte kann der Text »Pionierzy motoryzacji« (Pioniere der Motorindustrie) dienen (MD KB 7: 41). Ein kurzer Lesetext auf Polnisch informiert über die Familie Benz. Die anschließenden Aufgaben (auf Deutsch) schließen thematisch und vom Wortschatz her an die bisherigen Lektionsinhalte an (MD KB 7:41). Auch der Einstieg (MD KB 7: 41: ÜB 1) ist gelungen: dadurch, dass die Lerner darüber sprechen, welche 
Automarken aus Deutschland kommen, wird bereits vorhandenes landeskundliches Wissen aktiviert.

Ein anderes gelungenes Beispiel für integrative Landeskunde stellt die Vermittlung von Jahreszahlen dar (vgl. Abbildung 25). Die Zahlen werden hier am Beispiel von ausgewählten Erfindungen aus dem deutschsprachigen Raum vermittelt (MD KB 8: 57).

Die bewusste Auseinandersetzung mit landeskundlichen Inhalten wird auf den Projekt-Seiten (im Anhang) angeregt. Es handelt sich hierbei immer um projektartige Aufgaben, die die Lernenden dazu auffordern, eigenständig über bestimmte Phänomene der zielsprachigen Kulturen zu recherchieren und die Ergebnisse in Form von (multimedialen) Präsentationen mit den Mitschülern zu diskutieren.

Auch im Arbeitsbuch wird DACH-Landeskunde immer wieder aufgegriffen. Ähnlich wie im Lehrbuch ist sie zwar teils stereotypenhaft-verallgemeinernd (z. B. in der Zuordnungsaufgabe von Ländern und Speisen: "In Polen essen die Schüler Tomatensuppe mit Reis. In Österreich essen die Schüler Wiener Schnitzel.« MD AB 7: 59), was auf diesem Sprachniveau jedoch als akzeptabel gelten kann.

Der Einbezug der landeskundlichen Informationen erfolgt nicht nur durch explizite und implizite Inhalte, sondern auch durch Bilder. Bereits auf dem Cover des ersten Bands (sowohl Lehr-als auch Arbeitsbuch) wird zwar sehr typisch das Brandenburger Tor abgebildet, es handelt sich aber auf jeden Fall um ein ästhetisch sehr ansprechendes Foto (strahlend blauer Himmel, Sonnenstrahlen scheinen durch die Torgänge), das einen klaren Bezug zur Zielsprachen-Kultur aufweist. Genauso sind die in dem Lehrwerk abgebildeten Fotos insgesamt als ästhetisch sehr ansprechend zu bezeichnen. Die Bilder und Fotos transportieren zahlreiche kultur- und landeskundliche Informationen. So bilden die Fotos u. a. auch implizit eine multikulturelle Gesellschaft ab (z.B. auch Portraits von Personen mit asiatischem KB 7: 66-67 oder afroamerikanischem Hintergrund MD KB 7: 139-140, MD AB 7: 41), zeigen touristische Attraktionen wie das Märchenschloss in Neuschwanstein (MD KB 7: 130) oder Sehenswürdigkeiten Berlins (MD KB 7: 77, AB 7:49), Münchens (MD KB 7:113) und Wiens (MD KB 7: 95, $A B$ 7: 65). Die Fotos machen zum Teil auch gleichzeitig indirekt auf kulturelle Besonderheiten der zielsprachigen Länder aufmerksam (Ortsschilder in Deutschland MD KB 7: 33) und gewähren Einblicke unter die Haus- (MD KB 8: 48, 53, AB 8: 36) oder Schuldächer (MD KB 7:27).

Durch die Gestaltung - große Bilder zu Beginn jeder Einheit, Lesetexte mit Bildern und/oder Fotos illustriert - bekommt das Lehrwerk eher einen MagazinCharakter. Das dürfte den Lese- und Formatgewohnheiten der Zielgruppe entgegen kommen und für diese ansprechend wirken. 


\section{Deutsche Erfindungen}

1 Arbeitet in Gruppen. Lest die Texte und ordnet mündlich den beschriebenen Personen die richtigen Erfindungen zu.

Pracujcie w grupach. Przeczytajcie tekstyi przyporządkujcie ustnie opisanym osobom wlaściwe wynalazki.

Wilhelm Conrad Röntgen, ein deutscher Physiker, entdeckt 1895 die .... eine Revolution für die Medizin. Er bekommt dafür 1901 den Nobelpreis für Physik.

Johannes Gutenberg, ein deutscher Drucker, erfindet um 1440 den ... . und druckt als Erster auf der Welt die Bibel.

Levi Strauss (geboren in Deutschland als Löb Strauß), ein deutschamerikanischer Industrieller, erfindet 1873 die .... und verkauft diese Hosen an Goldgrăber im Westen Amerikas.

Carl von Linde, ein deutscher Professor und Ingenieur, erfindet 1876 den ersten .... .

Carl Friedrich Benz, ein deutscher Ingenieur und Automobilpionier, erfindet 1886 das erste moderne ... , das Benz Patent-Motorwagen Nummer 1 heilßt. Vincenz Prießnitz, Naturheiler aus Osterreichisch-Schlesien, ,.Wasserdoktor" genannt, therapiert seit dem Jahr 1826 Kranke nicht mit Medikamenten, sondern mit Wasser. Von seinem Namen Prießnitz kommt das polnische Wort

\section{Jeans}

\section{Dusche}

\section{Automobil}

\section{Buchdruck}

\section{Kühlschrank}

entdecken - odkrywać erfinden - wynajdować $\quad$ Erfinder, der - wynalazca

2 Lies die Texte in Aufgabe 1 noch einmal und ergänze mündlich die Sätze mit den richtigen Informationen. Przeczytaj teksty w zadaniu 1. jeszcze raz i uzupełnij ustnie zdania właściwymi informacjami.

1. Wilhelm Conrad Röntgen bekommt 1901 den Nobelpreis für .... .

\section{A. Medizin}

B. Physik

2. Johannes Gutenberg druckt um 1440 als Erster .... A. die Bibel

B. die Zeitungen

3. Levi Strauss verkauft 1873 ...hosen an Goldgräber. A. Plastik-

B. Jeans-
4. Carl von Linde erfindet 1876 den ersten .... .

A. Kühlschrank

B. Schrank

5. Das erste Automobil von Carl Friedrich Benz heißt .... .

A. Benz Patent-Motorwagen Nummer 10

B. Benz Patent-Motorwagen Nummer 1

6. Vincenz Prießnitz therapiert Kranke mit ... .

A. Wasser

B. Medikamenten

Schreibe die Jahreszahlen wie im Beispiel ins Heft. Napisz w zeszycie słownie określenie roku jak w przykładzie.

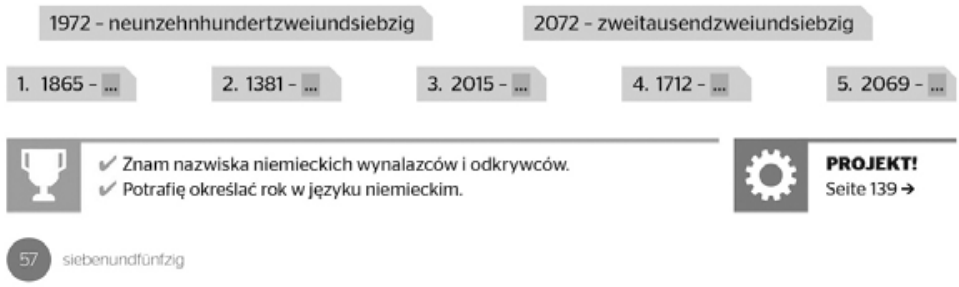

Abbildung 25: Einführung der Jahreszahlen als Beispiel für Sprach- und Landeskundeinhalte integrierende Aufgabe (Meine Deutschtour KB 8: 57) 


\subsubsection{Landeskundeansätze und Prinzipien in Meine Deutschtour}

Vielfalt der deutschen Kultur- und Sprachlandschaft

In dem Lehrwerk Meine Deutschtour wird großer Wert auf die Vermittlung von kultur- und landeskundlichen Informationen aus dem gesamten deutschsprachigen Raum gelegt. Bereits auf den ersten Lehrwerkseiten der beiden Bände in `Starter` wird überwiegend in Zahlen, großformatigen Bildern und kurzen Informationstexten die kulturelle Vielfalt des deutschsprachigen Raums präsentiert. Dadurch, dass der Lernbeginn mit landeskundlichen Informationen gestaltet ist, bekommt die zu lernende Sprache somit ein mehr oder weniger konkretes Gesicht. Auch die Relevanz der deutschen Sprache im europäischen bzw. weltweiten Kontext wird hier bereits am Anfang betont: »Für $24 \%$ der Bürger der Europäischen Union ist Deutsch die Muttersprache, 101 Millionen Menschen benutzen weltweit Deutsch als ihre Muttersprache, was den 12. Platz unter allen Sprachen der Welt ergibt, 14 Millionen Menschen lernen weltweit Deutsch als Fremdsprache« (vgl. Abbildung 26).

An diese Informationen anschließend folgen Seiten, auf denen eine DACHLLandkarte und die vier Hauptstädte der DACHL-Länder präsentiert werden. Die weiteren dieser insgesamt 14 landeskundlichen Seiten sind den einzelnen Ländern gewidmet: jeweils zwei Deutschland, Österreich, der Schweiz und eine Liechtenstein.

Sprachlich bleibt es durchweg beim Hochdeutschen. Bis auf Begrüßungen (MD AB 7:11) und typisches Essen (MD AB 7:59) werden keine dialektalen oder landessprachlichen Begriffe präsentiert, was hinsichtlich des Sprachniveaus nachvollziehbar erscheint.

\section{Der Bezug zur Lebenswelt der Zielgruppe}

Der Bezug zur Lebenswelt der Zielgruppe wird vor allem an den im Lehrwerk vermittelten Inhalten sichtbar. Es handelt sich um Themen und Inhalte aus der Alltagskultur: Familie, Schule, Feste oder Sport sind typische Beispiele dafür.

Im Lehrwerk gibt es wenige Aufgaben, bei denen die Lernenden dazu aufgefordert werden, die Inhalte mit der persönlichen Lebenswelt in Verbindung zu setzen. Das erfolgt mittels solcher Fragen, wie z. B. »Hast du ein Haustier? Wie ist es? « (MD KB 7: 127) oder »Was möchtest du in München besichtigen? Berichte.» (MD KB 7: 113).

Ihre persönlichen Erfahrungen oder Meinungen dürfen die Lernenden überwiegend in den Projekten präsentieren, die die Lektionen ergänzen. Da sich die Aufgaben im Anhang befinden, haben sie einen eher fakultativen Charakter. Als Beispiel kann das Thema Schule dienen. In dem die Lektion ergänzenden Projekt sollen die Schulwege in Deutschland recherchiert und in der Klasse präsentiert werden. In zwei darauf folgenden Aufgaben soll zunächst die Frage 


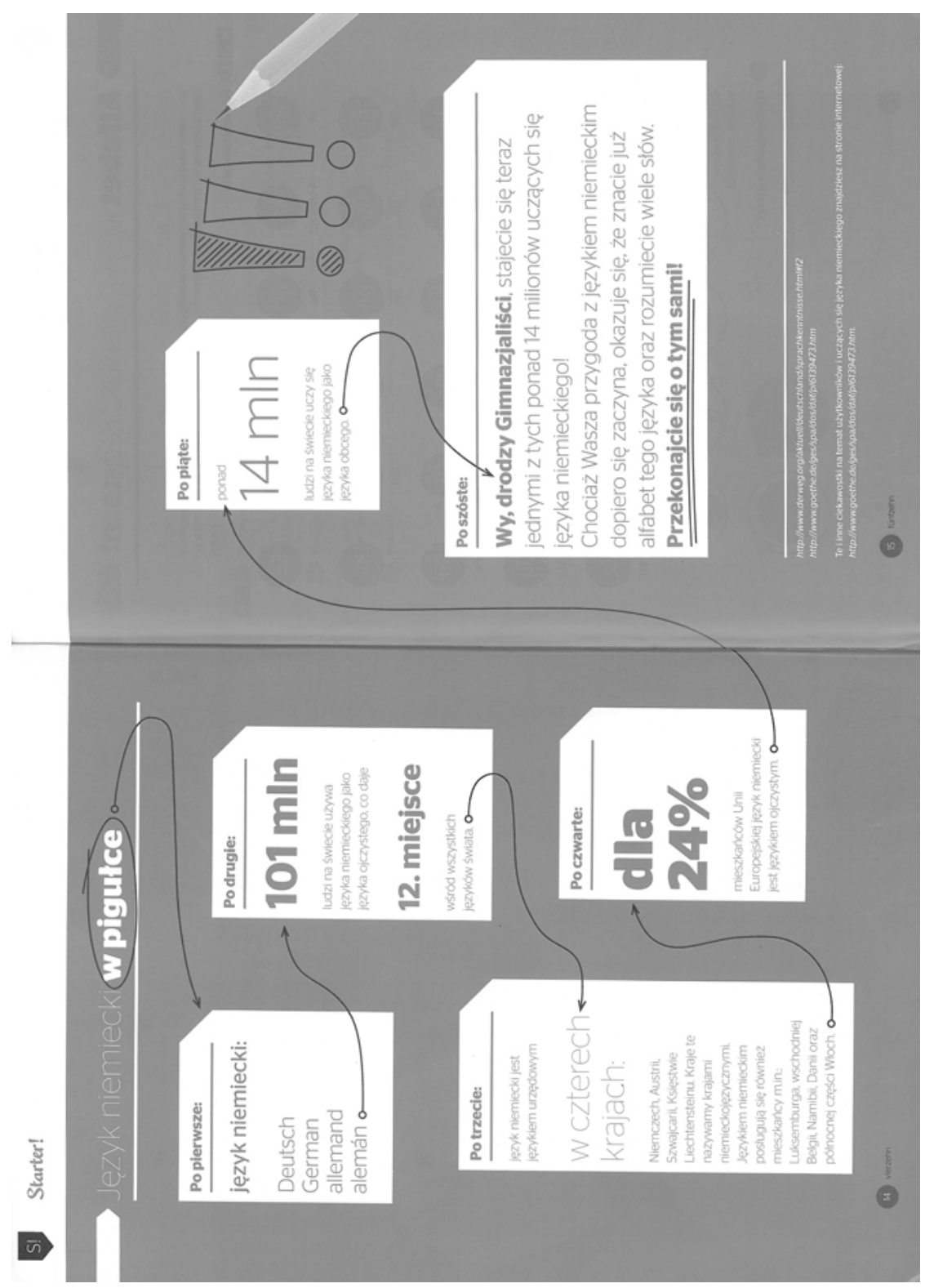

Abbildung 26: Relevanz der deutschen Sprache im europäischen bzw. weltweiten Kontext (Meine Deutschtour KB 7: 14-15) 
beantwortet werden, „Wie kommen Schüler aus eurer Klasse/Schule zur Schule? Womit fahren sie? Sammelt in Gruppen Informationen.« Anschließend sollen die Lernenden darüber berichten (MD KB 8: 141). Zu betonen beim Bezug zur Lebenswelt der Lernenden ist bei der Bewältigung der einzelnen Aufgaben die Anknüpfung an die Erfahrung mit dem Umgang der jungen Menschen mit den neuen Medien. Die Lernenden werden an vielen Stellen aufgefordert, die neuen Medien bei ihren Präsentationen zu nutzen oder sich auf ihre Erfahrung im Umgang mit den Medien zu stützen, wenn sie z. B. einen Blogeintrag zum Thema "Meine Lieblingsjahreszeit« (MD KB 8: 59) schreiben.

Am Ende des Arbeitsbuches befinden sich zusätzlich drei Filmlektionen, zu jedem Film gibt es entsprechende Übungen. Die an die Filmlektionen anschließenden Videoblog-Seiten enthalten Tipps und weiterführende Fragen, die den Lernenden helfen sollen, selbst einen Videoblog zu gestalten. Die einzelnen Kurzfilme knüpfen thematisch an die Inhalte der Einheiten an: In den Videoclips in Band 1 von Meine Deutschtour lernen die Lernenden den Jungen Chris und seine Familie (Film 1), seine Stadt (Film 2) und seine Hobbys (Film 3) kennen. In Band 2 betrifft das Filmmaterial `Marie und ihr Zuhause`, >Blanka und ihre Pflichten`, `Anne und ihre Stadt`. Die Arbeitsbücher werden jeweils von einer Seite mit QR-Codes zu weiteren didaktisierten Videoclips mit dazugehörigen Arbeitsblättern abgeschlossen: In Meine Deutschtour 7 gehören dazu: Besuch in München; Krautsalat - ein Kochrezept; Tour durch Bayern; Tour durch Berlin; Tour durch die Schweiz. Meine Deutschtour 8 bietet sechs weitere Filme und Übungsblätter zu folgenden Themen: Annett Louisan; Briefe an den Weihnachtsmann; Modetrends; Tour durch Norddeutschland; Tour durch Österreich; Tour durch Sachsen.

\section{Stimulierung einer kulturreflexiven Haltung}

Landeskundliche Inhalte stehen sehr oft in einem thematischen Zusammenhang mit der Lektionseinheit. Sie sind nicht nur altersgemäß und entsprechend den Interessen der Jugendlichen gestaltet, sondern regen auch zu einer Reflexion über die Ausgangskultur an. Meistens angeleitet durch kurze Fragen werden die Lernenden dazu aufgefordert, sich über ihre eigene Lebenswelt auszutauschen und anschließend darüber zu berichten. Auch der Einbezug der Ausgangskultur in der Zielsprache kommt in dem Lehrwerk nicht zu kurz. Neben DACH-Personen werden in dem Lehrwerk auch polnische Personen bzw. solche, die bzw. deren Familie aus Polen kommt und in DACH lebt, einbezogen. Z. B. Familienbezeichnungen am Beispiel des Fußballspielers Miroslav Klose (KB 7:38) sind nicht nur ein gutes Beispiel dafür, wie integrative Landeskunde aussehen kann, durch den polnischen Familienhintergrund wird - zumindest indirekt - auch ein Bezug zur Ausgangskultur hergestellt. 
Ein anderes Beispiel für die Stimulierung einer kulturreflexiven Haltung stellt die Lektion zu den Uhrzeiten dar (MD KB 7: 71, vgl. Abbildung 27). Die Einführung der Uhrzeiten wird mit einem internationalen Vergleich zum Thema begonnen: "Wann beginnt die Schule in den einzelnen Ländern?« (Brasilien, Deutschland, China, Großbritannien, Spanien), der mit der Frage abschließt: »Wann beginnt die Schule in Polen?» - Ein sehr einfaches Beispiel dafür, wie sprach- und landeskundliches Lernen integriert werden kann: der simple Vergleich unterschiedlicher Schulbeginn-Zeiten in verschiedenen Ländern mit der anschließenden Frage nach dem Heimatland macht ganz schnell deutlich, dass nicht alles überall gleich ist.

Dies wird nochmals explizit in der Dialogecke Extra aufgegriffen (MD KB 7: 78). Der kurze Dialog »Die Schule beginnt zu früh!« stellt ein Beispiel für einen vergleichenden landeskundlichen Ansatz dar, auch wenn die Lernenden dies hier rein rezeptiv erfahren und nicht explizit zur Auseinandersetzung damit angeregt werden.

Zur Reflexion laden die Aufgaben auf den Projekt-Seiten an. So sollen die Lernenden u. a. beim Thema Schule zunächst eine Präsentation über das Schulsystem und das Notensystem in Deutschland erstellen (MD KB 7: 141, vgl. Abbildung 28), dann eine Präsentation über das Schulsystem und das Notensystem in Polen. Anschließend werden sie dazu aufgefordert, die beiden Systeme zu vergleichen.

An einer anderen Stelle werden die Lernenden im Zusammenhang mit dem Thema Frühstück und Abendessen dazu angeregt, sich über die Essgewohnheiten der Polen auszutauschen (MD KB 7: 87). Bei dem Thema Feste sollen Informationen nicht nur zu Karneval in Deutschland, sondern auch in Polen gesammelt und präsentiert werden (MD KB 8: 140). Beim Thema Wohnen reflektieren die Lernenden darüber, "In was für Häusern wohnen die Deutschen? In was für Häusern wohnen die Schüler aus eurer Klasse/Schule?« (MD KB 8: 143, vgl. Abbildung 29).

\section{Erlebte Landeskunde}

Bei der Arbeit an kleinen projektartigen Aufgaben, die am Ende der beiden Bände vorgeschlagen werden, bekommen die Lernenden zusätzlich die Möglichkeit, selbst aktiv zu werden, d.h. landeskundliche Informationen eigenständig zu recherchieren und daran anschließend mit der von ihnen wahrgenommenen Realität der Ausgangskultur zu vergleichen. Die aktive Informationssuche und das in Beziehung setzen mit der eigenen Lebenswelt lässt die Lernenden nicht nur die einzelnen Länder und deren Bewohner aus dem deutschsprachigen Raum besser kennen lernen, sondern kann sich als wichtiger motivierender Faktor für das Lernen der deutschen Sprache erweisen. 


\section{Wann beginnt die Schule?}

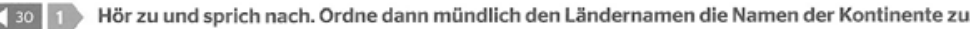
Posłuchaj i powtórz. Nastẹpnie przyporządkuj ustnie panstwom własciwe nazwy kontynentów.

Europa - Asien - Afrika - Nordamerika · Sũdamerika - Australien
1. Großbritannien - ...
2. Brasilien - .
3. China - ...
4. Frankreich - ....
5. Spanien - ...
6. Aggypten - ...
7. die USA - ...
8. Australien - ....

2 Wann beginnt die Schule in den einzelnen Ländern? Sieh dir die Uhrzeiten an und berichte darüber. O której godzinie zaczyna się szkoła / zaczynają się lekcje w poszczególnych krajach? Popatrz na obrazki przedstawiające zegary i opowiedz o tym.

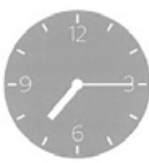

Brasilien

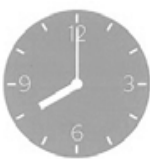

Deutschland

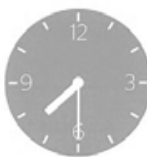

China

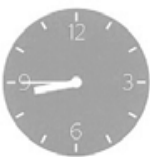

Großbritannien

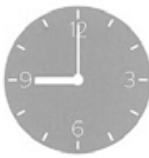

Spanien

- In Brasilien beginnt die Schule um ... .

- In Deutschland ... .

$>\ln \ldots .$.

Wann beginnt die Schule in Polen?

- In Polen beginnt die Schule meist um ....

3 Sieh dir das Foto auf der Titelseite des Kapitels an und beantworte mündlich die Fragen. Popatrz na zdjęcie zamieszczone na stronie tytulowej rozdziału i odpowiedz ustnie na pytania.

Wie spāt ist es in Lisbon? Wie viel Uhr ist es in New York?

Mach die Ausspracheũbung auf Seite 146.

Przejdż na stronę 146 i cwicz wymowę.

5 Ergänze mündlich die Fragen mit den angegebenen Fragepronomen. Uzupełnij ustnie pytania podanymi zaimkami pytającymi.

1. Entschuldigung. ... spăt ist es? - Tut mir leid, ich habe keine Uhr.

2. ... Uhr gehst du in die Schule? - Um halb acht.

3. ... kommst du nach Hause? - Am Nachmittag.

4. Georg. .... Uhr ist es? - Es ist zehn nach sieben.

Abbildung 27: Stimulierung einer kulturreflexiven Haltung am Beispiel des Themas Schulbeginn (Meine Deutschtour KB 7: 71) 


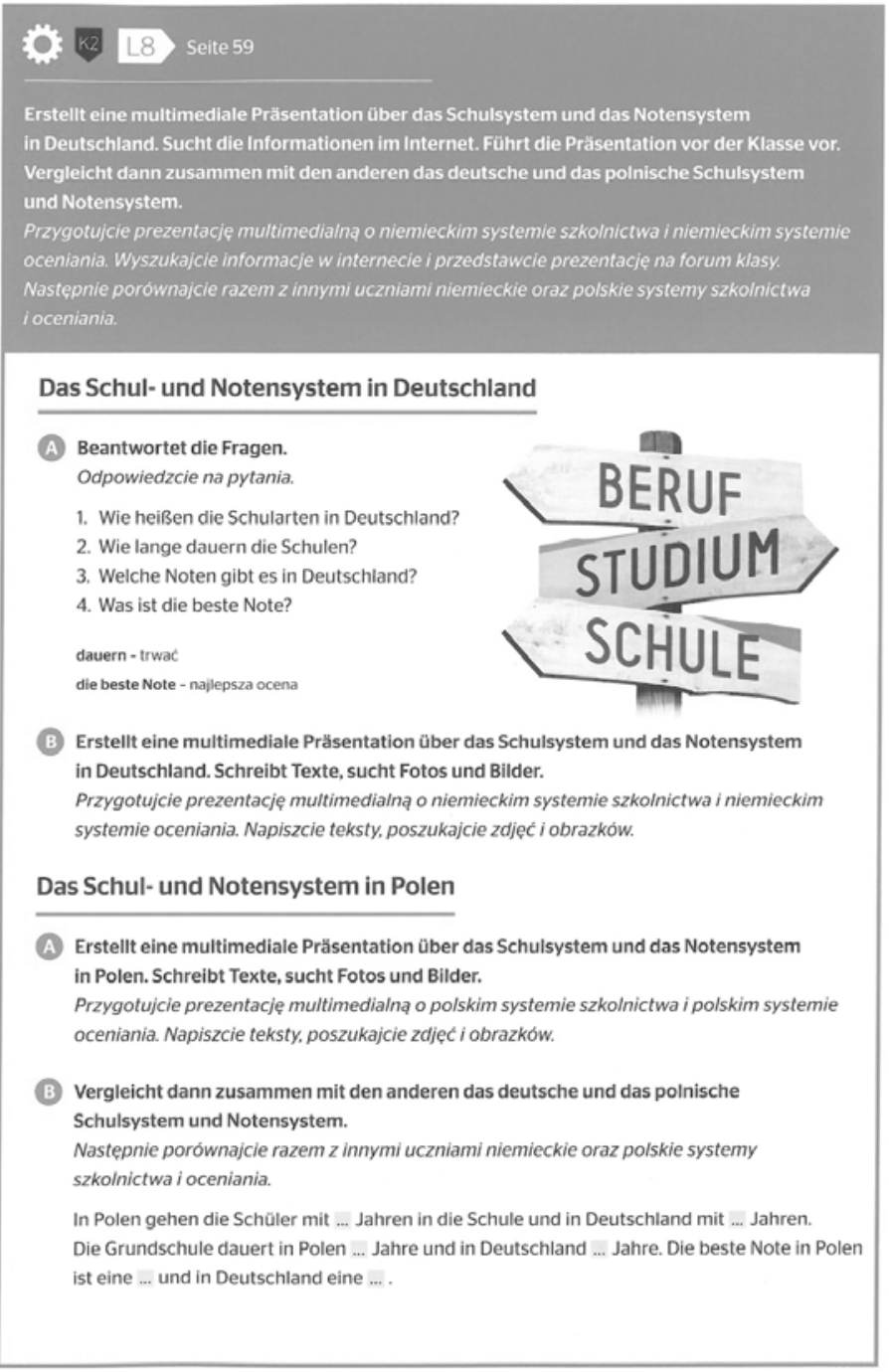

einhunderteinunavierzig

Abbildung 28: Stimulierung einer kulturreflexiven Haltung am Beispiel des Themas Schulsystem und Schulnotensystem (Meine Deutschtour KB 7: 141) 


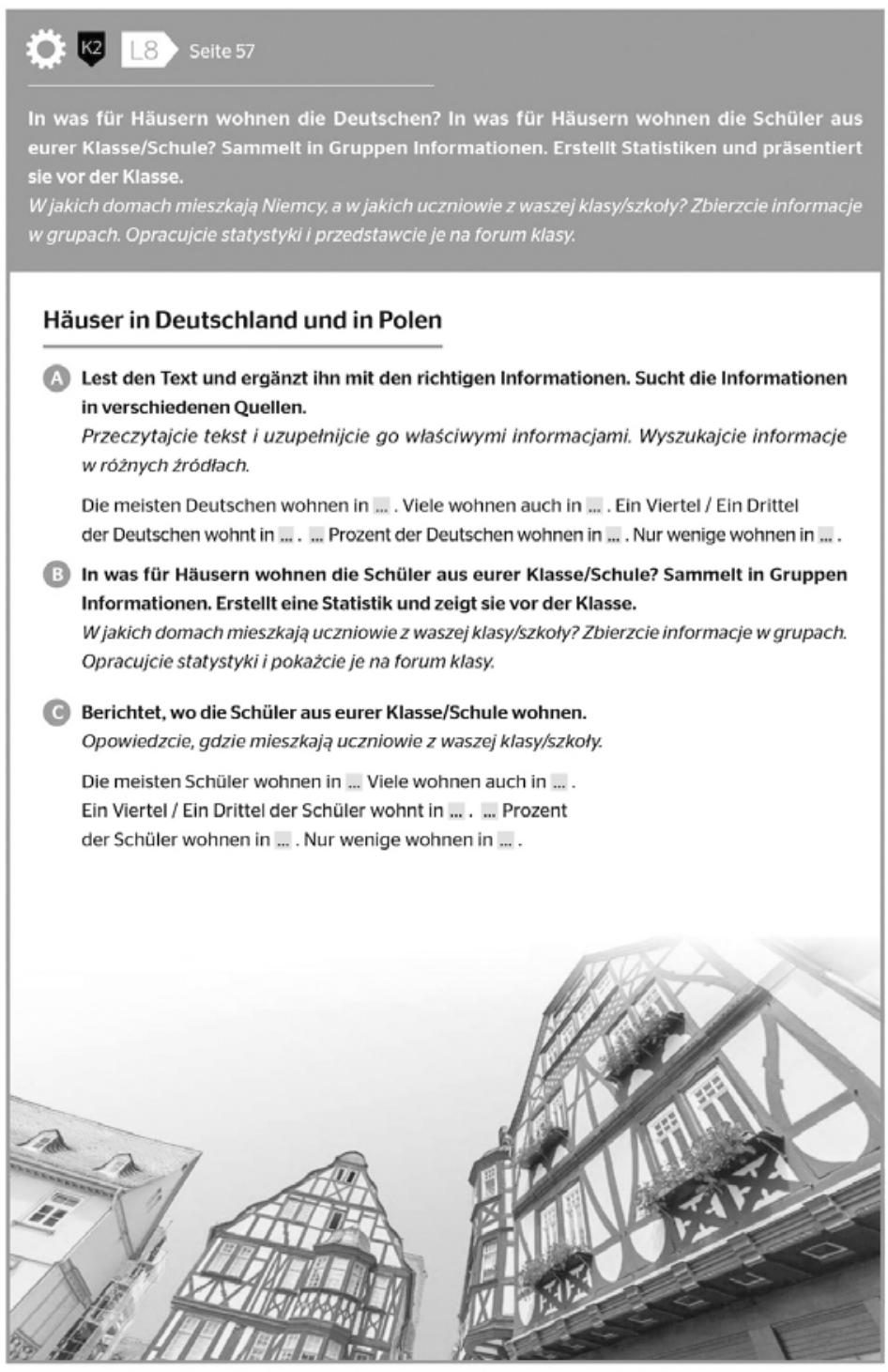

einhundertneununddreißig

Abbildung 29: Stimulierung einer kulturreflexiven Haltung am Beispiel des Themas Häuser in Deutschland und in Polen (Meine Deutschtour KB 8: 139) 


\section{Fazit}

Meine Deutschtour kann als positives Beispiel für ein Lehrwerk gesehen werden, das nicht nur ansprechend gestaltet ist, sondern auch in weiten Teilen gelungen Sprachlernen und Kultur/Landeskunde (und das auf A1-Niveau) zu integrieren weiß. Das Lehrwerk bietet ein ausgewogenes landeskundliches Angebot an Informationen aus verschiedenen DACH-Ländern. Besonders hoch zu werten, ist der gewählte Einstieg in eine fremde Sprache über die Landeskunde. Das Vormodul "Starter« bietet einen Zugang zu der deutschen Sprache. Die Lernenden erfahren zunächst, wo Deutsch gesprochen wird, lernen seine Position im weltweiten Kontext kennen. Die explizite Präsentation der vier deutschsprachigen Länder zu Beginn des Lernprozesses ermöglicht, dass die zu lernende Sprache nicht abstrakt und vor allem nicht kontextunabhängig initiiert wird. Im gesamten Lernprozess kommt der Landeskunde eine klar erkennbare Position zu. Landeskundliche Inhalte werden nicht isoliert dargestellt, sondern sind in das Thema der einzelnen Lektionen integriert. Das sprachliche und das landeskundliche Wissen werden somit nicht isoliert voneinander, sondern integrativ vermittelt. Das führt dazu, dass die Lerner sehr oft die Sprache über konkrete, alters- und interessengerechte Inhalte lernen.

In jeder Einheit wird projektorientiertes Lernen unter dem Titel >Projekte`ermöglicht. Das selbständige Weiterarbeiten und Vertiefen der kultur- und landeskundlichen Kenntnisse kann bestimmt nicht nur Erfolge schaffen, sondern auch zum Vergnügen am Lernen beisteuern. Auch die Anregungen zu eigenen Blogs weisen einen hohen Lernerbezug auf. Der Lernende steht mit seinen Interessen und Erfahrungen im Zentrum.

\subsubsection{Fazit: Polnische Lehrwerke}

Die Analyse der polnischen Lehrwerke für den DaF-Unterricht zeigt, dass obwohl es sich hier um ein elementares (A0/A1) Niveau handelt, landeskundliche Inhalte Einzug in die fremdsprachliche Praxis erhalten. Den Interessen und Bedürfnissen der Lernenden entsprechend gewähren die Lehrwerke Einblicke in die kulturelle und sprachliche Vielfalt des deutschsprachigen Raums. Die Landeskunde wird in den analysierten Lehrwerken überwiegend implizit umgesetzt und weist einen thematischen Zusammenhang mit dem Lektionsinhalt auf, wobei hier faktisches Wissen und die kommunikative Alltagshandlungsfähigkeit als Lernziele leitend sind.

In den von uns analysierten Lehrwerken gibt es auch gesonderte Stellen für Informationen und Aufgaben mit beabsichtigtem Landeskundefokus. Hier werden die Inhalte meist explizit dargestellt und/oder die Lernenden werden dazu angeregt, bestimmte landes- und kulturkundliche Informationen eigen- 
ständig zu recherchieren. Zahlreiche der analysierten Aufgaben stellen ein gutes Beispiel dafür da, wie Landeskunde bereits zu Lernbeginn in den Unterricht integriert werden kann. Die Vermittlung der landeskundlichen Inhalte geht über die reine Informationsvermittlung hinaus und versucht, die Lernenden durch aktive Teilnahme (Fragestellungen, Recherche-Aufgaben, projektartige Aufgaben) in den Lernprozess einzubeziehen. Die Position dieser Inhalte - am Ende jeder zweiten Einheit (in Kompass Team) oder am Ende des Buches (Projektartige Aufgaben in Mit Links! und in Meine Deutschtour) - verleiht dem DACHPrinzip unnötig den Status eines additiven Extras.

Die Themen und die Wahl der Inhalte sind im Allgemeinen in allen drei von uns analysierten Lehrwerken als alters- und interessenentsprechend der Zielgruppe zu bezeichnen. Ebenso ist in allen drei Lehrwerken der kommunikative Ansatz klar erkennbar.

Ein klarer landeskundlicher Fokus macht sich in Meine Deutschtour bemerkbar. Ein ausgewogenes Verhältnis an implizit und explizit vermittelten Inhalten trägt zur kommunikativen Alltagshandlungsfähigkeit unter den Lernenden bei. Mit projektähnlichen Aufgaben werden die Lernenden zu eigenständigen Recherchen angeregt, die dann als Anlass für kritische Auseinandersetzung mit den Inhalten dienen. Mit dem Einsatz von kurzen Filmen über den Alltag von Jugendlichen im deutschsprachigen Raum werden Einblicke in die gegenwärtige zielsprachige Kultur gewährt.

Insgesamt kann als Fazit festgehalten werden, dass die Lernenden trotz des elementaren Sprachniveaus mit der kulturellen und sprachlichen Vielfalt des deutschsprachigen Raums vertraut gemacht werden. Auch wenn dies auf Anfängerniveau nur in einem begrenzten Umfang geschehen kann, stellen die zu vermittelten Inhalte eine gute Grundlage für die weitere Beschäftigung auf höheren Lern- und Niveaustufen dar. 
Open-Access-Publikation im Sinne der CC-Lizenz BY-NC-ND 4.0

() 2020, Vandenhoeck \& Ruprecht GmbH \& Co. KG, Göttingen

ISBN Print: 9783847111214 - ISBN E-Lib: 9783737011211 


\section{Good practice}

Anschließend an die Analysen der Vermittlung landes- und kulturkundlicher Inhalte in in Polen und in den Niederlanden produzierten DaF-Lehrwerken und entsprechend der dabei befolgten Analysekriterien werden in diesem Kapitel einige Beispiele für die Umsetzung unterschiedlicher landes- und kulturkundebezogener Lernziele präsentiert, die als Anregung dienen können, wie solche Lernziele in der Unterrichtspraxis umgesetzt werden könnten. Die Übersicht erhebt keinen Anspruch auf Vollständigkeit.

Vorgestellt werden Aufgaben aus Materialien und Lehrwerken für den Grundstufenbereich (A1-B1), die dem DACH-Prinzip entsprechend die kulturelle und sprachliche Vielfalt des deutschen Sprachraums zeigen und erfahren lassen (Abschnitt 5.1), die entsprechend der Kontaktdidaktik Lernenden die Möglichkeit bieten, Verbindungen zwischen dem vermeintlich fremden und eigenen kulturellen Raum zu entdecken (Abschnitt 5.2) sowie Aufgaben, in denen angestrebt wird, eine kulturreflexive Haltung bei Lernenden zu fördern und es den Lernenden ermöglicht wird, am zielsprachigen Diskurs teilzunehmen (Abschnitt 5.3). Aufgaben, die Erlebte Landeskunde zentral stellen und den Lernenden ermöglichen, die zielsprachige Kultur direkt und affektiv erfahren zu können, werden nicht gesondert präsentiert, da diese - wie auch unsere Analysen gezeigt haben - in Lehrwerken für Deutsch als Fremdsprache, gedacht für den Einsatz außerhalb des deutschsprachigen Raums, kaum Berechtigung haben, und wenn, dann nur mit Hilfe digitaler Projekte oder Rechercheaufgaben umgesetzt werden können. Aktivitäten, die zielsprachige oder -kulturelle Aspekte in der eigenen Umgebung aufgreifen und so Landeskunde erleben lassen, finden sich unter Abschnitt 5.3. 


\subsection{Kulturelle und sprachliche Vielfalt des deutschen Sprachraums}

Zahlreiche DaF-Lehrwerke steigen mit Landkarten bzw. Umrissen der DACHLänder ein oder präsentieren diese in (einer) der ersten Lektion(en). Im Folgenden werden einige der zahlreichen, möglichen Varianten und dazu gehörenden Aktivitäten präsentiert.

Als Einstieg in Band 3 präsentiert Team Deutsch (B1) eine Übersichtskarte der DACHL-Länder, der Flaggen und weitere faktische Informationen zugeordnet werden sollen. Auch wenn Transfer und Anwendung für die Lernenden hier durch die Lehrperson bzw. die Schüler selbst erfolgen muss, handelt es sich im Sinne der faktischen Landeskunde um eine Aufgabe, die plurikulturelle Vielfalt und Unterschiede im deutschsprachigen Raum sichtbar macht (vgl. Abbildung 30).

Eine ähnliche, deutlich einfachere Aufgabe, die einen Einstieg für die Präsentation plurikultureller Vielfalt bieten kann, zeigt Prima Plus (Band A1.1), wobei Fotos typischer Sehenswürdigkeiten aus größeren DACH-Städten einer Umrisskarte der DACH-Länder zugeordnet und mit passendem Stadtnamen beschriftet werden sollen.

Rätsel: Länder und Städte

a) Wie heißen die Länder und die Städte? Die Landkarte vorne im Buch hilft.

b) Welches Foto zeigt welche Stadt? Die Buchstaben helfen.

Beispiel 2: Rätsel: Länder und Städte (aus: Jin/Rohrmann 2016: 11)

Demgegenüber wird in Band 2 von Team Deutsch (A2) versucht, zur DACHKarte auch persönliche Bezüge der Lernenden zu den DACH-Ländern herzustellen, indem u.a. nach Kontakten zu den DACH-Ländern, eigenen Aufenthalten oder Wunschzielen gefragt wird (vgl. Abbildung 31).

Auch in Entdeckungsreise D-A-CH, einem Kursbuch zur Landeskunde (A2B1) wird ein Einstieg mittels Landkarte gewählt, hier jedoch unter Einbezug auch anderer deutschsprachiger Regionen über die DACH-Länder hinaus, wie Luxemburg, Belgien und Südtirol. Pro Land bzw. Region wird hier in Informationskästen neben einer Abbildung der Flagge und des Länderkennzeichens angegeben, welche Sprachen Amtssprachen sind.

Die deutschsprachigen Länder und Regionen

a) Sehen Sie sich die Karte und die Informationskästen an.

b) Sprechen Sie miteinander über die deutschsprachigen Länder und Regionen.

Beispiel 3: Die deutschsprachigen Länder und Regionen (aus: Pilaski et al. 2013: 6) 


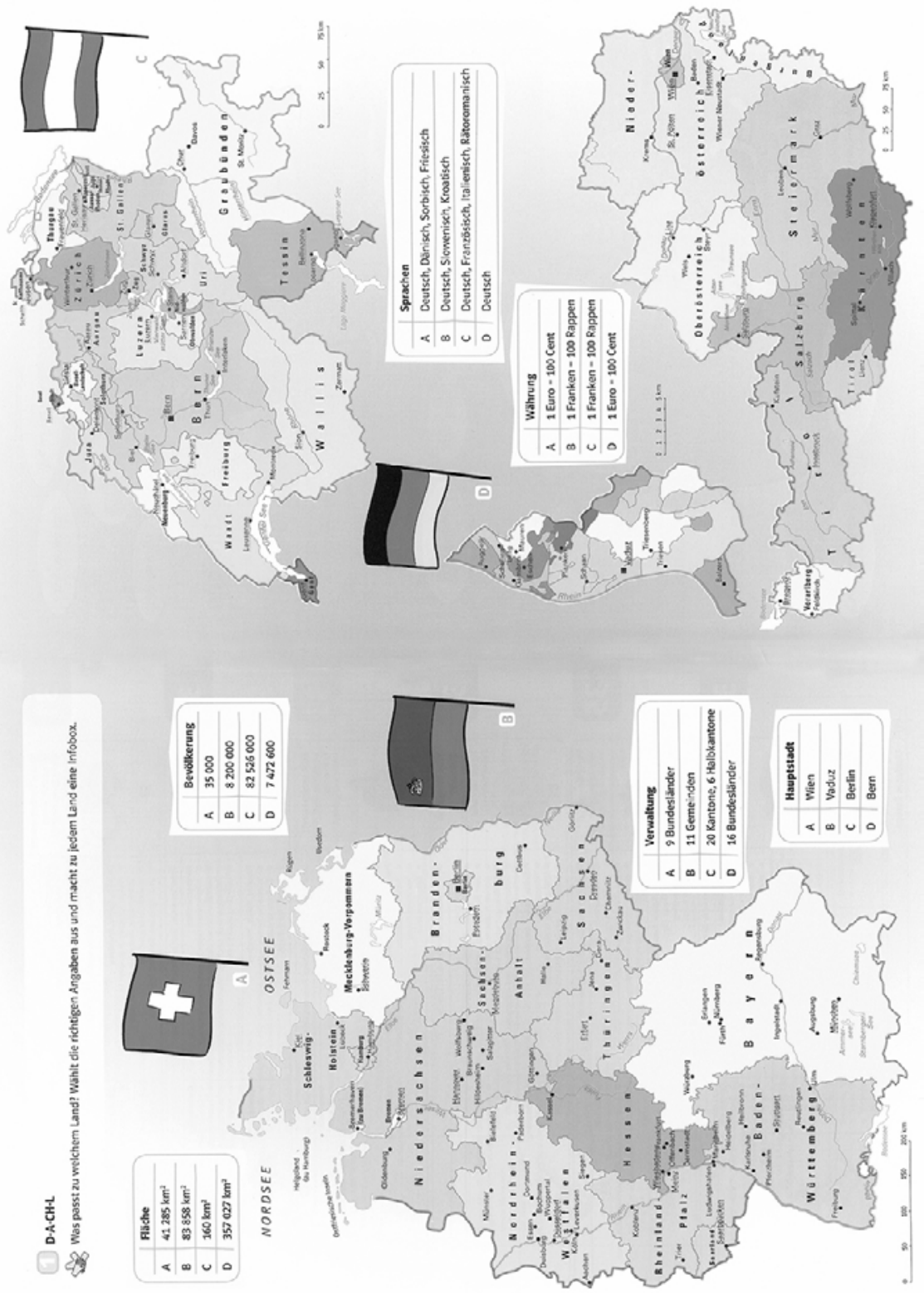

Abbildung 30: D-A-CH-L (aus: Esterl et al. 2016: 6-7) 


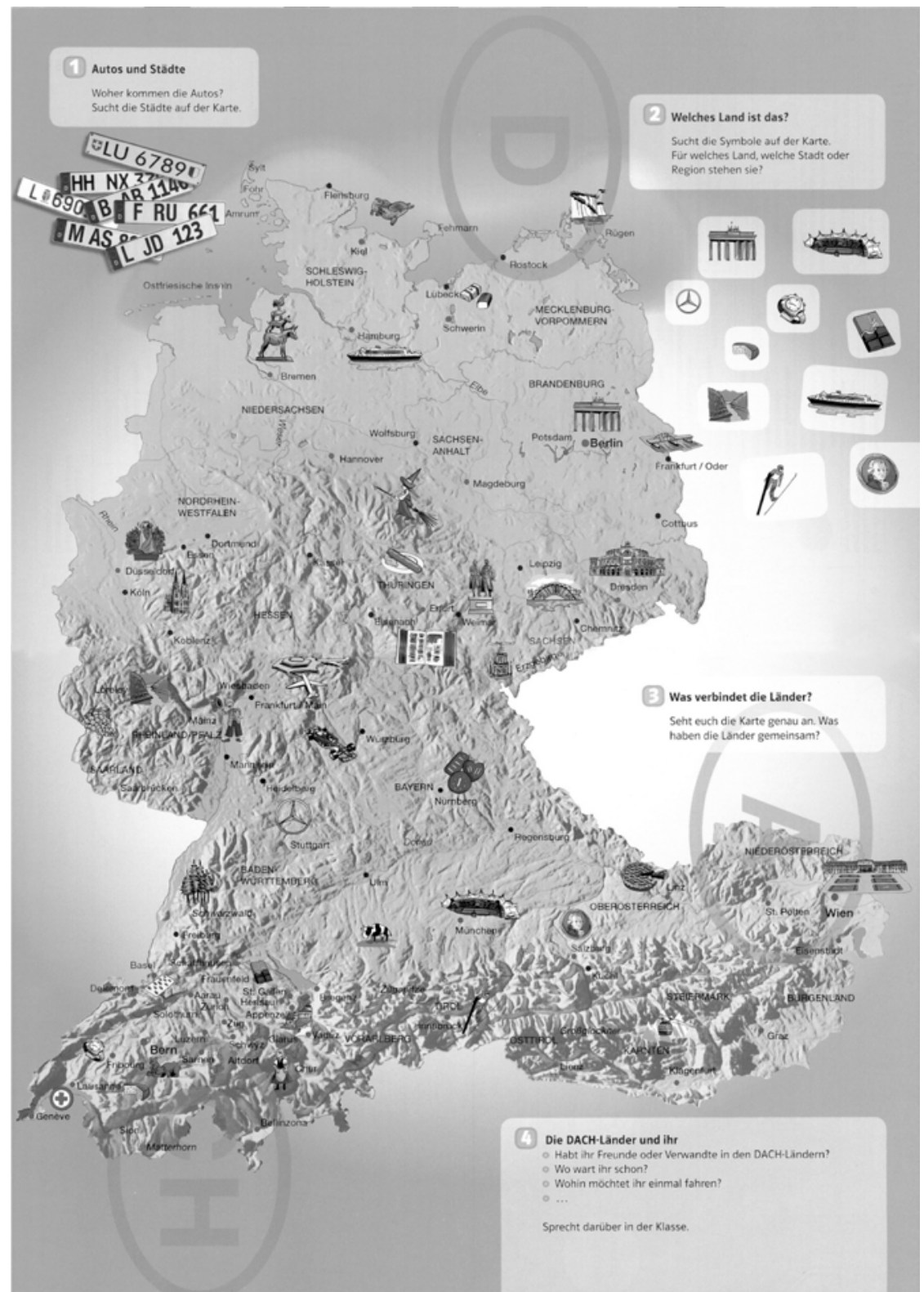

Abbildung 31: DACH-Länder (aus: Esterl et al. 2015: 6-7) 
An diese Karte schließen sich weiterführende Informationen zu Deutsch als Amtssprache in verschiedenen Ländern an. Nach einem Lesetext zu diesem Thema wird auf Gemeinsamkeiten und Unterschiede der deutschen Sprache in DACH fokussiert (vgl. Abbildung 32). Hierüber hinaus wird in Entdeckungsreise $D-A-C H$ der Blick für die DACH-Länder weiter geöffnet, indem auch Bezug auf sprachenpolitische Aspekte (u. a. Verankerung der Sprache im Grundgesetz (vgl. Abbildung 33) sowie Integrationspolitik (vgl. Pilaski et al. 2013: 16) genommen wird. Im Gegensatz zu den stark faktisch orientierten Seiten zur deutschen Sprache, kann hier durch die abschließenden Diskussions- und Debattieraufgaben auch eine Reflexion bei Lernenden initiiert werden, die jedoch teils viel Vorwissen erfordert - z.B. aktuelle sprachenpolitische Themen im eigenen Land - und ohne weiteren Input bei Schülern nicht als selbstverständlich vorausgesetzt werden kann.

Die sprachliche Vielfalt der DACH-Länder wird häufig mit Hilfe von Songtexten in verschiedenen Standardvarianten oder Dialekten in Lehrwerke integriert, wie beim folgenden Beispiel aus Zugspitze (Band 4 vwo). In diesem Beispiel wird den Lernenden auf A2-Niveau ein authentischer Text in liechtensteinischem Deutsch präsentiert und anschließend in einer Übersetzungsaufgabe mit der deutschen Standardsprache, d.h. kontrastiv, verglichen (vgl. Abbildung 34). Auf diese Weise können die Schüler entdecken, dass Deutsch mehr ist als die Hochsprache, die sie im Deutschunterricht erlernen.

In Lehrwerken finden sich zum Thema Musik auch Beispiele für Aufgaben mit offenem Charakter, wie in Klasse! A2:

Musik

Kennt ihr deutschsprachige Lieder oder Bands und Sänger/Sängerinnen aus

Deutschland, Österreich und der Schweiz? Erzählt.

Beispiel 4: Musik (aus: Fleer et al. 2019: 50)

In Deutsch echt einfach A1 wird sprachliche Vielfalt im deutschsprachigen Raum bereits auf absolutem Anfängerniveau über einen kleinen Lesetext präsentiert. Interessant ist die abschließende Aufgabe (Projektecke) zu Sprachen in den Nachbarländern der Lernenden, die verdeutlichen kann, dass sprachliche Vielfalt überall zugegen ist (vgl. Abbildung 35). 


\section{Die deutsche Sprache in Deutschland, Österreich und der Schweiz}

1 a Wie verstehen Sie diesen Spruch?

b Was wissen oder vermuten Sie über Gemeinsamkeiten und Unterschiede der deutschen Sprache in (DACH)? Sprechen Sie miteinander und machen Sie sich Notizen.

„Deutsche, Österreicher und Deutschschweizer sind getrennt durch ihre gemeinsame Sprache."

c ordnen Sie zu.

die gesprochene Sprache • das Hochdeutsch • die Mundart • das Schriftdeutsch * die Schulsprache • die Umgangssprache

1. das Hochdeutsch
$\begin{aligned} & \text { 2. } \\ & \text { 3. }\end{aligned}$
$\begin{array}{ll}\text { 4. } & \text { das Standarddeutsch } \\ \text { 5. } & \text { : die Sprache, die man in der Schule lernt } \\ \text { 6. } & \text { : die informelle Sprache, die man mit Freunden benutzt }\end{array}$
: der Dialekt

d Lesen Sie den Text. Welche Aspekte, über die Sie gerade gesprochen haben, finden Sie im Text wieder? Welche nicht? Was überrascht Sie?

$$
\text { -Deutsch }=\text {-Deutsch }=\text { 다-Deutsch? }
$$

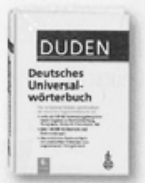

Deutschland, Österreich und deutsch oder Standarddeutsch. die Deutschschweiz haben eine Zwar gibt es landestypische Varigroße Gemeinsamkeit: die anten des Standarddeutschen, vor deutsche Sprache. Aber ist das allem im Wortschatz und in einiDeutsch, auf das man in den gen Besonderheiten der Grammadrei Ländern trifft, auch tat- tik, des Satzbaus und der Orthosächlich ein und dieselbe Spra- grafie, aber diese Unterschiede che? Eine seltsame Frage? behindern die schriftliche Kom-

Überhaupt nicht! Denn warum gibt es in (DACH) munikation über die Ländergrenzen kaum. unterschiedliche deutsche Wörterbücher und

Sprachführer? Und warum werden wohl in der Schweiz spezielle Kurse und Lehrwerke für Schweizerdeutsch angeboten?

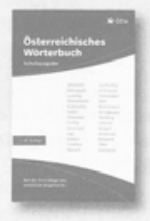
Um diese Fragen zu beantwor- Findruck ist gar nicht so falseh, denn es handelt ten, muss man zuerst eine wichti- sich beim gesprochenen Umgangsdeutsch in den ge Unterscheidung machen: zwi- verschiedenen deutschsprachigen Regionen sehr schen dem geschriebenen und oft nicht um Hochdeutsch, sondern um unterdem gesprochenen Deutsch. Die schiedliche deutsche Dialekte oder Regionalspra. deutsche Schriftsprache, die man chen, die sich in sehr vielen Aspekten voneinander in (DACH) in der Schule lernt, der und vom Standarddeutschen unterscheiden. Desman in der Presse und Literatur halb muss man sich auch nicht wundern, dass selbst der drei Länder begegnet und die deutsche Muttersprachler an fremden Orten im auch im politischen und wirt- deutschsprachigen Raum mit der mündlichen Allschaftlichen Leben aktiv verwendet wird, ist in der tagskommunikation auf Deutsch häufig große Tat im Wesentlichen ein und dasselbe Hoch- Schwierigkeiten haben!

das Schweizerdeutsch (auch Schwizerdütsch oder Schwiizertüütsch) = Name für die Dialekte in der Deutschschweiz die Variante $=$ eine andere Form

8

Abbildung 32: Die deutsche Sprache in Deutschland, Österreich und der Schweiz (aus: Pilaski et al. 2013: 8) 


\section{Sprachenpolitik}

1 a Was verbinden Sie mit dem Wort "Sprachenpolitik"? Sammeln Sie.

b Welche sprachlichen Themen könnte oder sollte ein Staat politisch regeln? Ergänzen Sie die Liste.

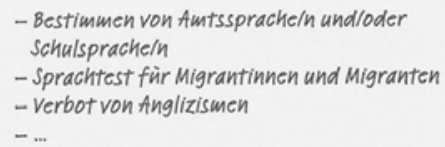

Ablehnung und Unverständnis für Initiative rov rziehungsministerin

Neue vorschläge der österreichischen Boundern:

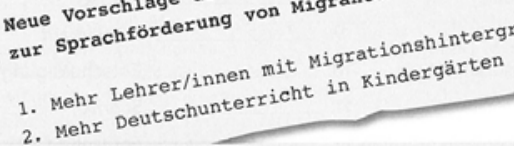

Die Bundesversammlung - Das Schweizer Parlament

Kampf gegen die Verwendung von Anglizismen

Fragen:

1. Was meint der Bundesrat generell zur Verwendung von Anglizismen in der Bundesverwaltung und in den vom Bund kontrollierten Firmen?

2. Hat der Bundesrat eine Meinung zu der Bezeichnung „Postmail” für die Briefkästen der Schweizer Post?

c Führen Sie eine parlamentarische Debatte über ein sprachenpolitisches Thema von Ihrer Liste.

A Wählen Sie ein Thema aus und formulieren Sie es genau.

B Bilden Sie mindestens zwei Parteien.

$C$ Legen Sie in jeder Partei die gemeinsame Meinung zum Thema fest und sammeln Sie passende Argumente.

D Führen Sie eine parlamentarische Debatte.

E Stimmen Sie am Ende ab.

d Welche aktuellen sprachenpolitischen Themen gibt es in Ihrem Land und was meinen Sie dazu? Sprechen Sie miteinander.

Bei uns sollen jetzt alle Kinder in der ersten Klasse der Grundschule Englisch als Fremdsprache lernen. Ich bin total dagegen. Die Kinder müssen doch erst ihre Muttersprache richtig lernen.
$\mathrm{Ja}$, findest du? Das sehe ich nicht so problematisch. Bei uns ist das große Thema der neue Sprachtest, den alle Einwanderer machen müssen. Da gibt es Proteste von allen Seiten. Ich selbst...

14

Abbildung 33: Sprachenpolitik (aus: Pilaski et al. 2013: 14) 


\section{Aufgabe 5 Musik aus Liechtenstein}

Natürlich mögen die Liechtensteiner auch gerne Musik. Rääs ist zum Beispiel eine bekannte Gruppe. Sie singt im Liechtensteiner Dialekt. Dieser Dialekt hat Ähnlichkeiten (gelijkenissen) mit dem Dialekt, der im Westen von Österreich gesprochen wird und dem Schwyzerdütsch aus der Schweiz.

\begin{tabular}{|c|}
\hline Weder dahaam \\
\hline $\begin{array}{l}\text { 1. Strophe: Müad uf dr Haamfahrt noch ma stränga Tag } \\
\text { Spür mine Knocha spür jeden Schlag } \\
\text { Jeder Atemzog bräännt wia Füưr } \\
\text { ha hōt alles ge und nüt isch mehr z tūür }\end{array}$ \\
\hline $\begin{array}{l}\text { Refrain } 1 \text { bi haam ko } \\
\text { haam i mils Land } \\
\text { döt wohne zfreda bi } \\
\text { bil weder dahaam } \\
1 \text { bi hasm ko } \\
\text { haam i miss Land } \\
\text { döt wohne zfreda bi } \\
\text { bil weder, weder dahaam }\end{array}$ \\
\hline $\begin{array}{l}\text { 2. Strophe: a Umaarmeg inneg söll se si } \\
\text { ganz tüüf dinna för immer im Härz dri } \\
\text { andleg bin } 1 \text { zrock i minna aagna Wänd } \\
\text { noch ra langa Reis wia schö miars zämma Händ }\end{array}$ \\
\hline Refrain \\
\hline $\begin{array}{l}\text { 3. Strophe: } 1 \text { well me fühla, wia du di fühlscht } \\
\text { trockne dine Trääna, wännd mol brüülscht } \\
\text { wänn mol down bisch, recht i de weder uff } \\
\text { ufs zämma lacha, i freu me ganz fescht druuf. }\end{array}$ \\
\hline Refrain \\
\hline
\end{tabular}

A Hör dir im Internet das Lied 'Weder dahaam' von Rääs an, lies mit und kreuze die richtige Zusammenfassung an.

\begin{tabular}{|l|l|l|l|l|}
\hline Zusammenfassung & 1. Strophe & Refrain & 2. Strophe & 3. Strophe \\
\hline $\begin{array}{l}\text { A Ich bin nach Hause gekommen, in } \\
\text { mein Land, wo ich zufrieden bin und } \\
\text { wo ich daheim bin. }\end{array}$ & & & & \\
\hline $\begin{array}{l}\text { B Nach einem anstrengenden Tag } \\
\text { komme ich müde nach Hause. Alles } \\
\text { tut mir weh. }\end{array}$ & & & & \\
\hline $\begin{array}{l}\text { C Eine feste Umarmung, endlich bin } \\
\text { ich wieder in meinen eigenen vier } \\
\text { Wänden nach einer langen Reise. }\end{array}$ & & & & \\
\hline $\begin{array}{l}\text { D Ich möchte wieder fühlen können und } \\
\text { deine Tränen trocknen, wenn du dich } \\
\text { schlecht fühlst. }\end{array}$ & & & & \\
\hline
\end{tabular}

B Such im Liedtext die liechtensteinische Übersetzung der folgenden deutschen Wörter und schreib sie auf.

\section{Strophe}

1. müde:

2. Feuer:

3. nichts:

\section{Strophe}

1. für:

2. endlich:

3. Reise:

\section{Refrain}

1. zufrieden:

2. wieder:

3. daheim:

\section{Strophe}

1. fühlen:

2. Tränen:

3. auf:

Abbildung 34: Musik aus Liechtenstein (Blok et al. 2017: 7-8) 


\section{Landeskunde}

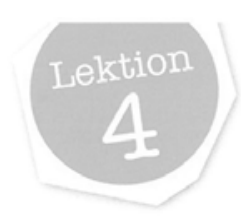

1 Richtig (R) oder falsch (F)? Lies den Text und kreuze an.

\section{Die deutschen Dialekte}

In Deutschland, Osterreich und der Schweiz spricht man nicht immer dasselbe. Es gibt auch sehr, sehr viele Dialekte. Und die sind manchmal gar nicht leicht zu verstehen. Hessen hat allein über 600 Variationen von Hessisch! In Frankfurt sagt man nicht: „Guten Tag!” Man sagt: „Guude!" In Hamburg heißt das: „Moin!" In der Schweiz hört man meistens: „Grüezi!" Und in Österreich: „Servus!” Rund um Wiesbaden sagt man nicht „sprechen”, sondern „babbele”. Die Hamburger sagen dafür aber „klönen” oder „schnacken”. Das „Handy" heißt in der Schweiz „Natel” und statt "Fahrrad" sagt man „Velo". Ganz schön schwer, oder? Keine Angst: Die meisten Menschen in den deutschsprachigen Ländern können nicht nur einen Dialekt, sondern auch Hochdeutsch. Das ist ein Deutsch ohne Dialekt.

1. In Hessen gibt es 600 Sprachen.

2. Es gibt viele Variationen für "Guten Tag!"

3. Die Schweizer haben andere Wörter als die Deutschen.

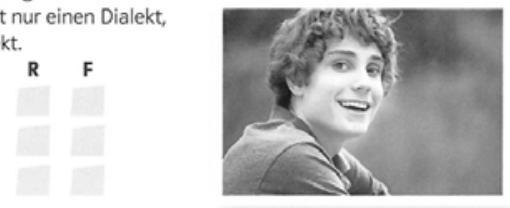

2 Woher kommen die Personen? Finde Informationen im Text und antworte.

Zeig auf der Landkarte, wo die Personen wohnen.

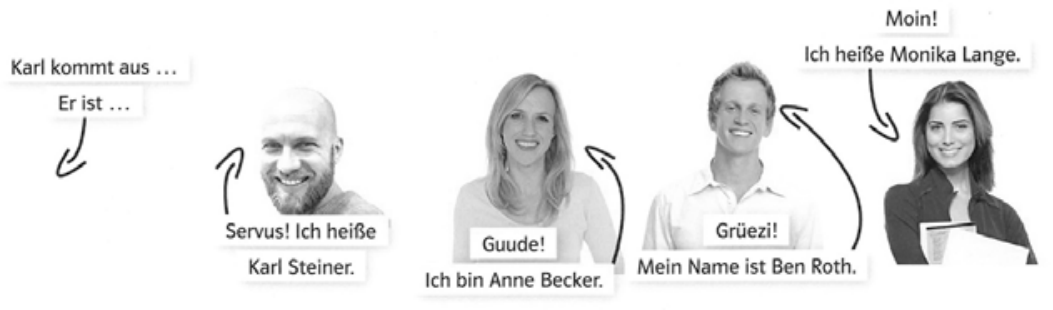

\section{Projektecke Sprachen in meinen Nachbarländern}

An welche Länder grenzt dein Land? Welche Sprachen sprechen die Menschen dort? Wie begrüßen sie sich? Was sagen sie zum Abschied? Recherchiert und sammelt Informationen.

\begin{tabular}{l|l|l|l} 
Die Nachbarländer & Die Menschen sprechen dort: ... & Ich begrüBe sie: ... & Zum Abschied sage ich: ... \\
\hline & & &
\end{tabular}

57

siebenundfünfzig

Abbildung 35: Landeskunde: Die deutschen Dialekte (aus: Motta 2016: 57) 
Eine andere Möglichkeit, die Vielfalt der DACH-Länder zu vermitteln und dabei DACH visuell zu erfassen, zeigt folgendes DACH-Memo-Spiel aus dem Materialpaket DACH-Erkundungen: Die deutschsprachigen Länder kennenlernen, das im Rahmen des Interregprojektes »Nachbarsprache \& buurcultuur « entstanden ist (vgl. Abbildung 36a und 36b).

DACH-Memo-Spiel
Aufgabenbeschreibung:
Das Memory beinhaltet Objektkarten aus Deutschland, Österreich und der Schweiz. Pro
Objekt gibt es also drei verschiedene Abbildungen, also beispielsweise ein Briefkasten
aus Deutschland, einer aus Österreich und einer aus der Schweiz.
Gespielt wird wie beim bekannten Memory, allerdings werden jeweils 3 (und nicht wie
üblich zwei) Karten aufgedeckt, gültig sind auch nicht Pärchen, sondern Drillinge. Wer
am Ende die meisten Drillinge hat, hat gewonnen.
Es ist wichtig, dass die Schüler und Schülerinnen sich während des Spielens über die
Bilder und ihre Eindrücke hierzu austauschen; sie sollen sich darüber unterhalten,
welches Bild aus welchem Land stammt und warum sie das denken. Dies gern nach Ende
des Spiels auch im Plenum auflösen.
Weiterführende Aufgabe:
Als weiterführende Aufgabe können die Schüler - z. B. via Internet-Recherche oder mit
Hilfe von Partnerschülern aus DACH - ihr eigenes DACH-Memory gestalten, inspiriert
von ihren ganz eigenen DACH-Eindrücken und persönlichen Interessen.

Beispiel 5: DACH-Memo-Spiel: Aufgabe (aus: Jentges/Holtland 2018: 9)

Team Deutsch (Band 3, B1) greift das sicher für den Fremdsprachenunterricht nicht unumstrittene Thema Klischees auf und präsentiert solche über die DACH-Länder. Diese werden im gleichen Moment relativiert, da sie nicht nur benannt, sondern auch in Frage gestellt werden: "Was meint ihr? «. Dass hieran anschließend Klischees zum eigenen Land gesammelt werden sollen, ermöglicht eine Relativierung von Klischees, da davon auszugehen ist, dass die gefragten Autostereotype zum eigenen Land Anlass geben, auch die für die DACH-Länder vorgegebenen $\mathrm{zu}$ hinterfragen. Die im Weiteren präsentierten, von (zukünftigen) Austauschschülern an ihre Partnerschüler im deutschsprachigen Raum geschriebenen kurzen Texte mit Fragen ermöglichen es, dass auch die Lernenden ermutigt werden, ihre eigenen Fragen zum deutschsprachigen Raum zu formulieren (vgl. Abbildung 37).

Auch Dimensionen greift in allen 3 Bänden Typisches aus den DACH-Ländern auf, dann allerdings - wie in Abbildung 38 aus Dimensionen. Magazin 2 (A2) u. a. mit einem Adventskalenderhaus und Schultüten zu sehen - als Rätselbilder, die die Lernenden sich spielerisch entschlüsseln sollen und hierdurch angeregt werden, über Gebrauch und Verwendung oder Nutzen dieser DACHDinge nachzudenken. Dass hierbei automatisch eigenkulturelles Wissen und entsprechende Vergleiche einbezogen werden, liegt auf der Hand. 


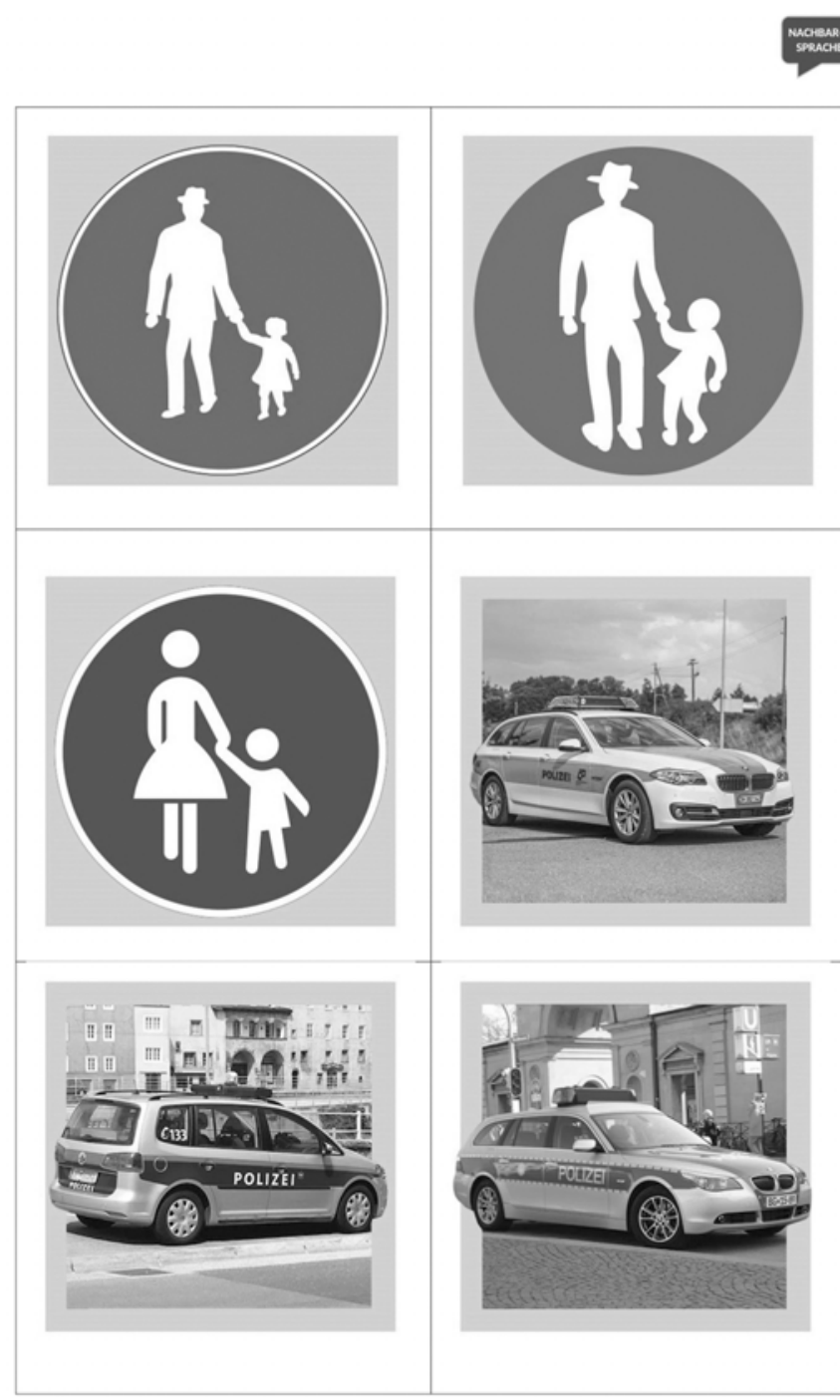

11

Abbildung 36a: DACH-Memo-Spiel: Spielkarten (aus: Jentges/Holtland 2018: 12) 


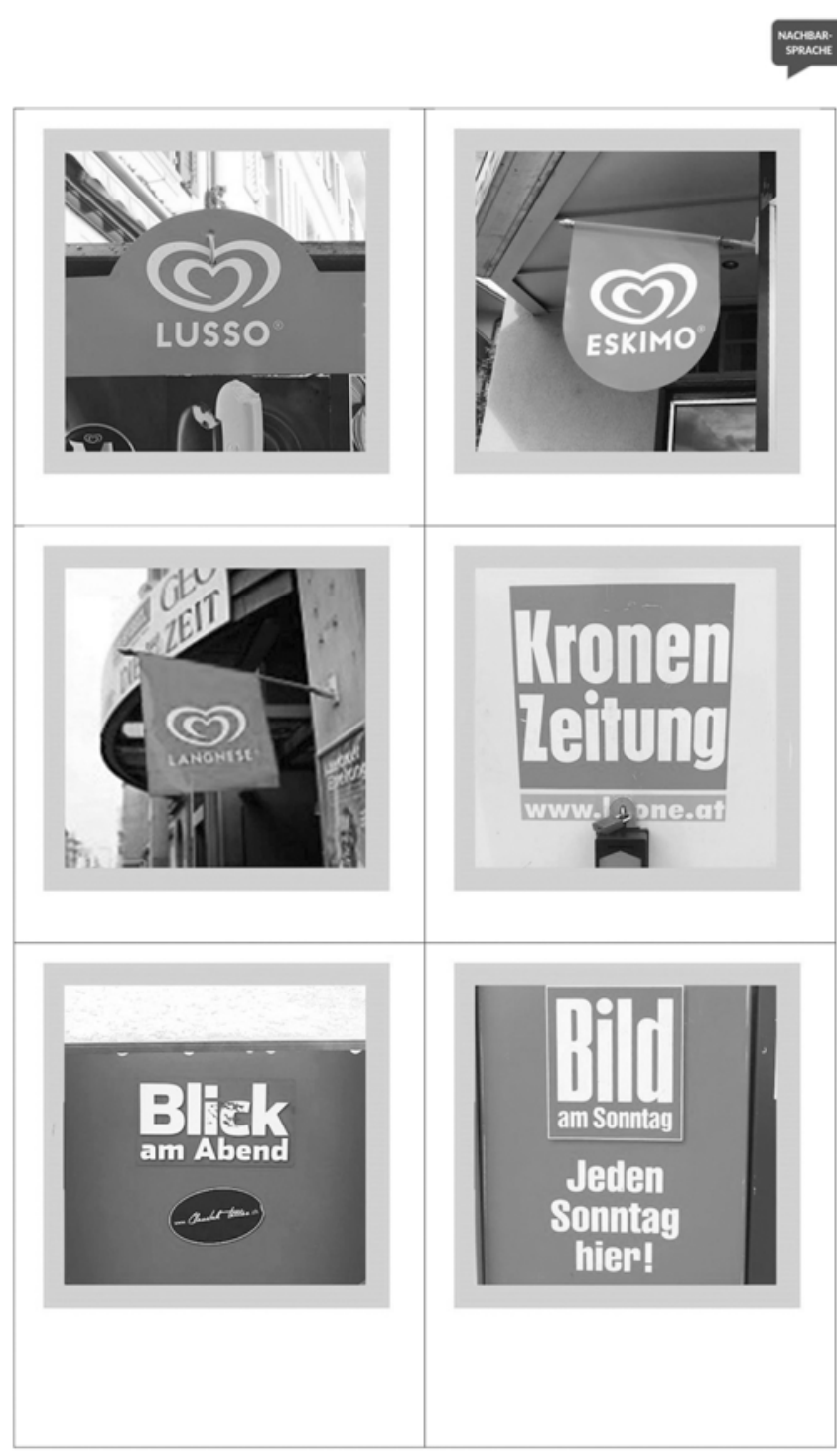

12

Abbildung 36b: DACH-Memo-Spiel: Spielkarten (aus: Jentges/Holtland 2018: 12) 


\section{g: Typisch DACH?}

- Ich glaube nicht, dass die Deutschen so viel...

\section{Ob das stimmt?}

a Im Deutschunterricht haben Jugendliche über Klischees zu DACH diskutiert und ihre Fragen aufgeschrieben. Was meint ihr dazu?
- Ich denke, dass alle Schweizer...

- Ich weiß nicht, ob die Österreicher...

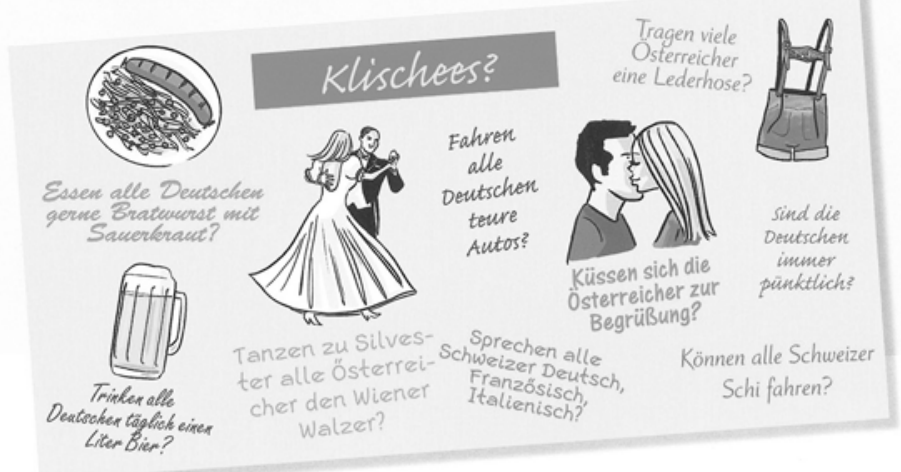

b Gibt es ähnliche Klischees über euer Land? Sammelt sie.

4 Austauschschüler fragen nach

a Lies die E-Mail-Ausschnitte. Zu welchem Problem haben die Austauschschüler Fragen? Nenne das Thema.

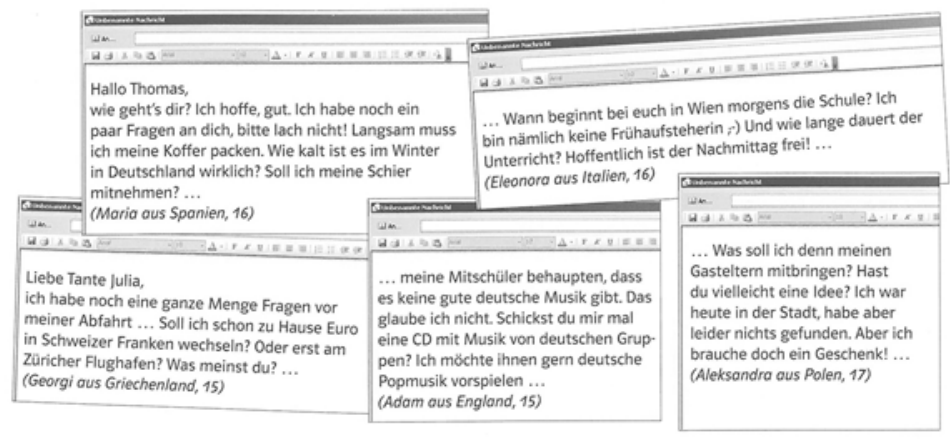

10 zehn

Abbildung 37: Typisch DACH? (aus: Esterl et al. 2016: 10) 


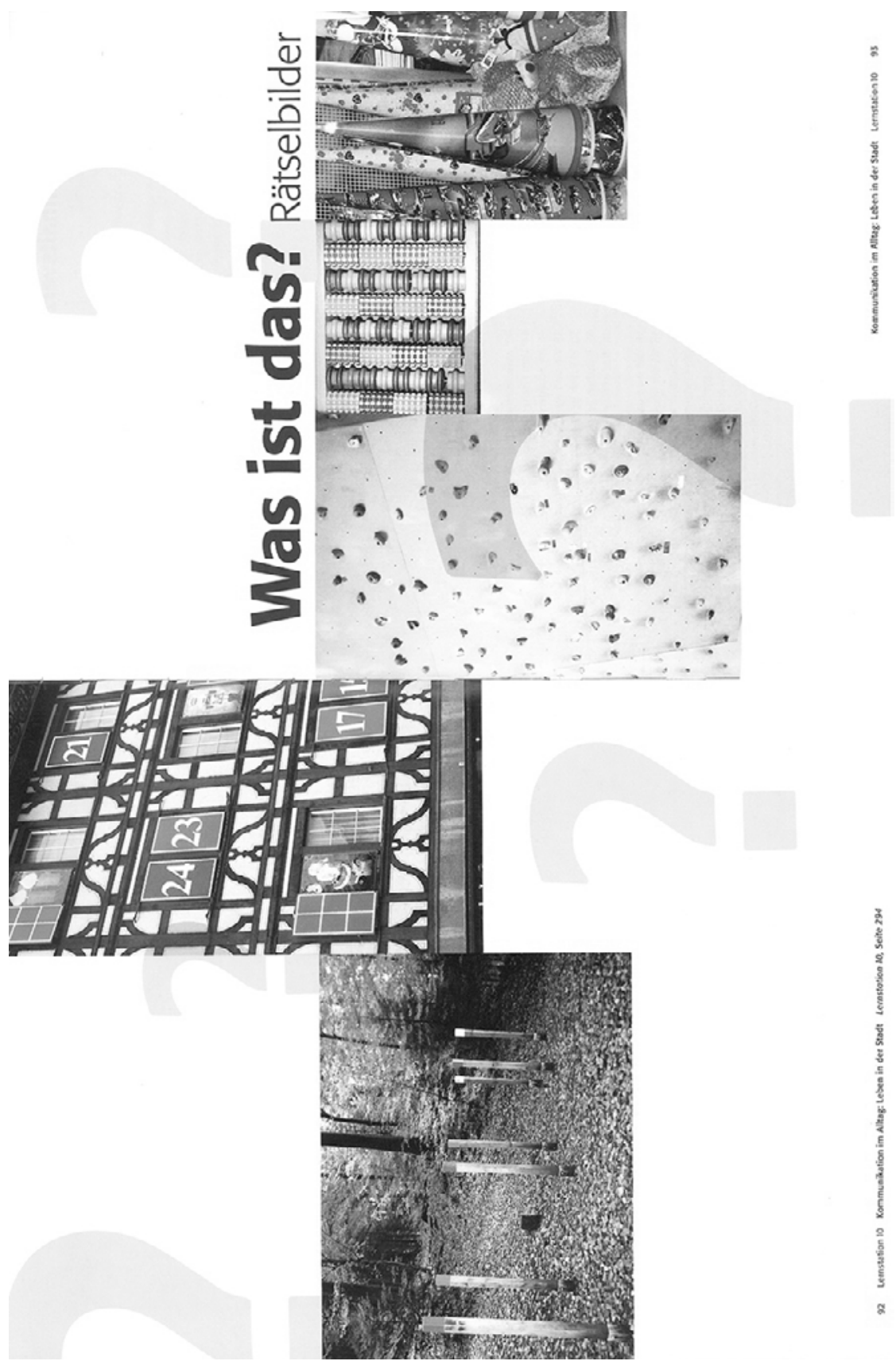

Abbildung 38: Was ist das? Rätselbilder (aus: Jenkins et al. 2003a: 92-93) 
Dass Vielfalt auch auf regionaler Ebene innerhalb eines Landes präsentiert werden kann, zeigt der Lesetext aus Klasse! A1 (vgl. Abbildung 39). Transfer auf die eigene Umgebung wird durch die abschließende Frage: „Was kann man bei euch machen?« ermöglicht.

Regionale und DACH-Vielfalt verknüpft präsentiert Dimensionen. Magazin 2 (A2) unter dem Titel "Städte und Landschaften" mit einer Fotocollage (vgl. Abbildung 40). Die hierzu in den Lernstationen (Dimensionen. Lernstationen 6-10) präsentierten Aufgaben laden Lernende nicht nur dazu ein, neben visuellen auch akustische Eindrücke (1, Beispiel 6) mit den Fotos zu verbinden, sondern auch persönliche Bezüge (2b), d), e), Beispiel 6) einzubeziehen:
1) Bilder und Klänge
Hören Sie Klangbilder: Welches Klangbild passt zu welchem Bild?
2) Bilder aus Deutschland, Österreich, der Schweiz und Liechtenstein Mini-Gruppe: Diskutieren Sie.
a) Welche Bilder passen nicht zu den DACH-Ländern? Warum?
b) Welche Bilder passen auch zu ihrem Heimatland. Warum?
c) Welche Bilder passen nur zu Deutschland? Warum?
d) Ihre Lieblingsbilder. Warum?
e) Austausch in der Klasse: Suchen Sie Personen mit den gleichen Lieblingsbildern.

Beispiel 6: Städte und Landschaften (aus: Jenkins et al. 2003b: 35-36) 


\section{Landeskunde}

7 a Am Meer und in den Bergen - Lest und ergänzt die Texte von Beele und Basti.

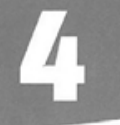

$\downarrow$ lecker $\downarrow$ duschen $\downarrow$ Berg $\downarrow$ Winter $\uparrow$ allein $\$$ kaputt $\$$ so sü $\beta \uparrow$ gefährlich $\downarrow$
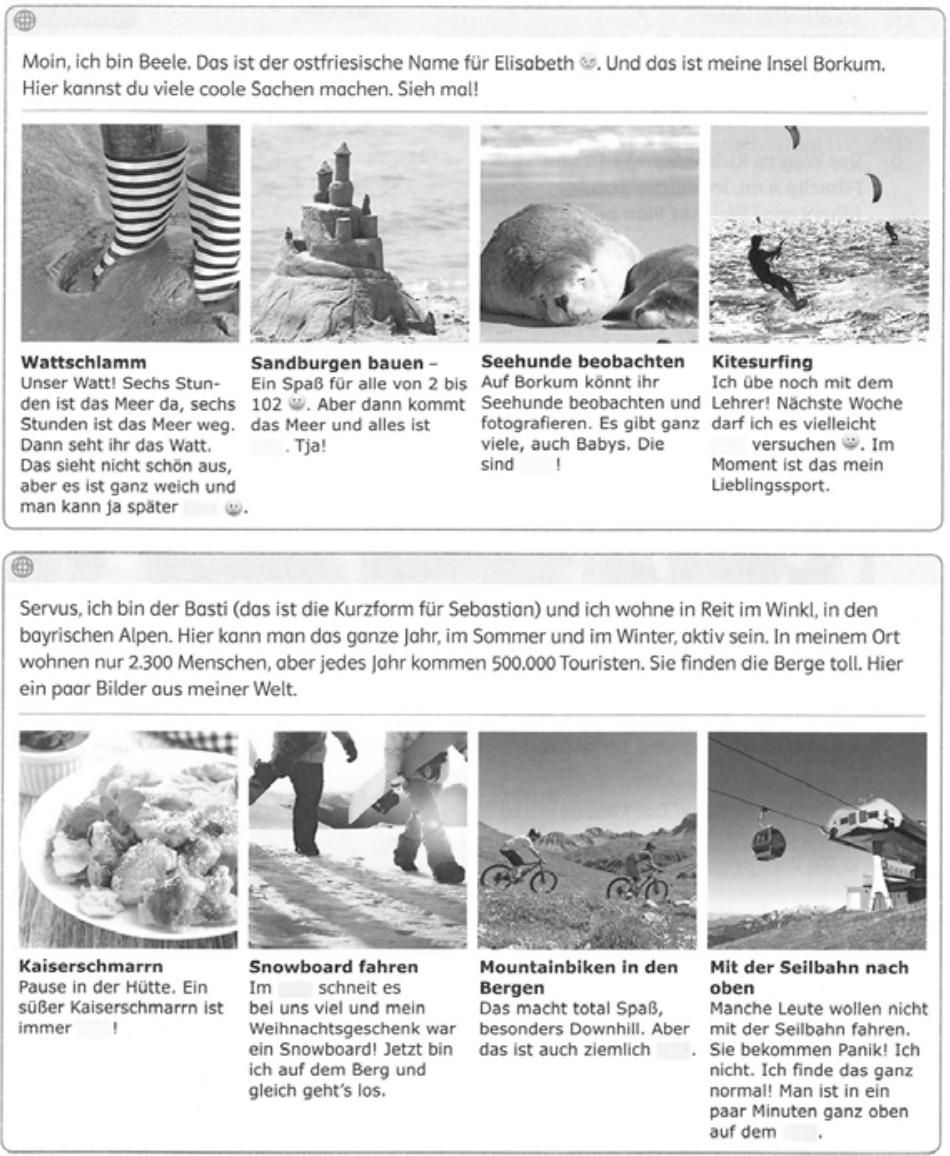

b Wohin wollt ihr lieber fahren: in die Berge oder ans Meer? Was wollt ihr dort machen? Sprecht zu zweit.

c Was kann man bei euch machen (Essen, Sport, Tiere, ...)? Sprecht in der Klasse.

Abbildung 39: Landeskunde: Am Meer und in den Bergen (aus: Fleer et al. 2018: 125) 


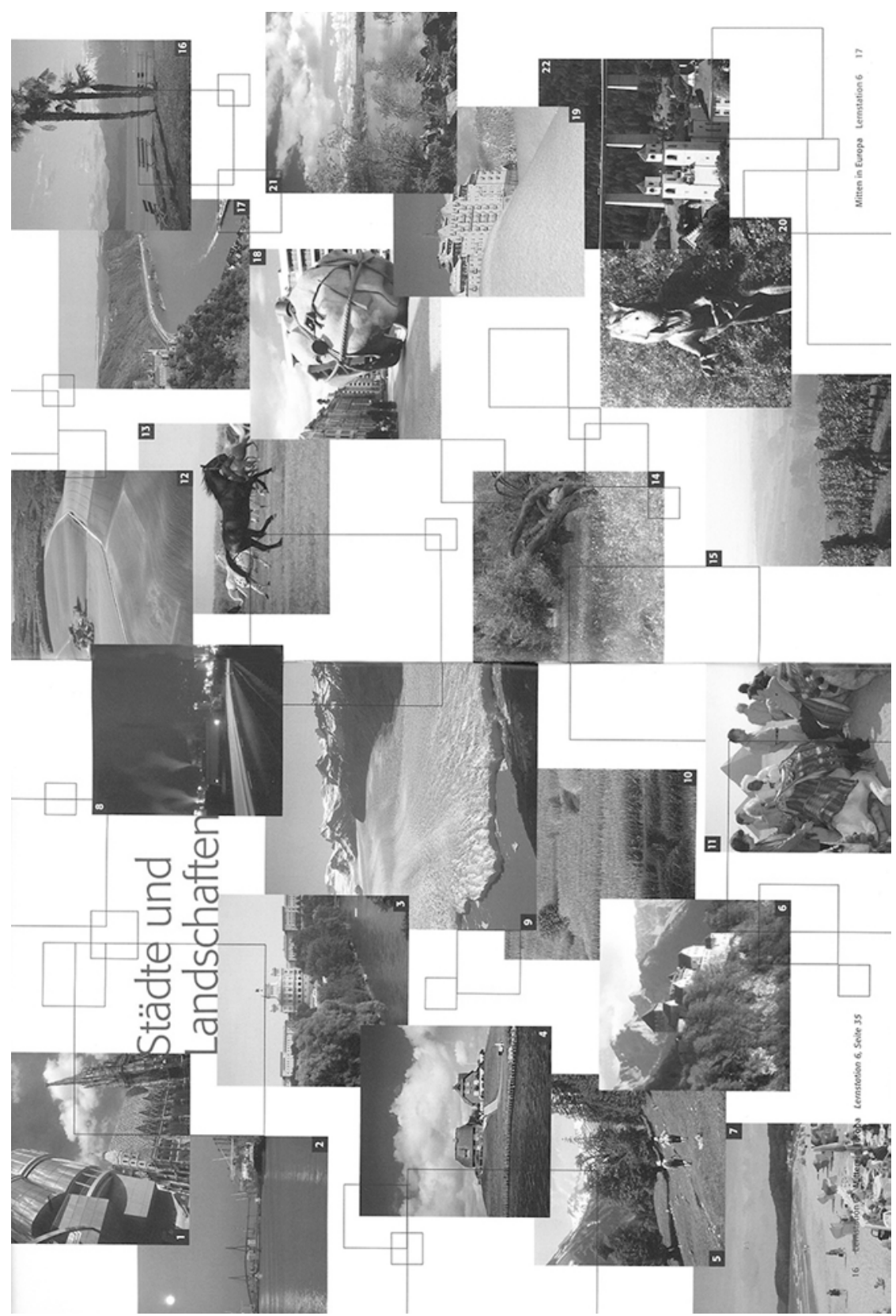

Abbildung 40: Städte und Landschaften (aus: Jenkins et al. 2003a: 16-17) 


\title{
5.2 Kontaktdidaktik: Verbindungen zwischen dem vermeintlich fremden und eigenen kulturellen und sprachlichen Raum entdecken
}

Einen einfachen, wenn auch nicht ausschließlich als landeskundlich zu bezeichnenden Einstieg ins Deutschlernen bietet Deutsch echt einfach A1 gleich zu Beginn des Deutschlernens.

\author{
Warum lernt ihr Deutsch? \\ Arbeitet in Gruppen. Schreibt in der Muttersprache die Gründe. Dann sammelt die \\ Ergebnisse und macht eine Klassenstatistik.
}

Beispiel 7: Warum lernt ihr Deutsch? (aus: Motta 2016: 17)

Beste Freunde A1.1 steigt mit dem eigenen Land ein, wodurch beim Deutschlernen direkt Bezüge zur eigenen Umgebung hergestellt werden. In diesem Beispiel ist die Aufgabenstellung sprachlich so vorstrukturiert, dass ein Plakat vom eigenen Land bereits auf absolutem Anfängerniveau in der Zielsprache angefertigt werden kann (vgl. Abbildung 41).

Zahlreiche Lehrwerke bieten bereits auf Grundstufenniveau Aufgaben an, die dazu anregen, den eigenen Wohnort zu erkunden oder zu präsentieren, wie etwa in Band A1.1 von Prima Plus, wenn anschließend an ein Video über Sehenswürdigkeiten in Berlin nach Sehenswertem in der eigenen Stadt gefragt wird (Beispiel 8) oder in Band A2.2 dieses Lehrwerks, wenn weiterführend nach einer Recherche zu Flüssen im deutschsprachigen Raum (Elbe, Donau, Mosel, Main) eine Sehenswürdigkeit aus der eigenen Umgebung für deutschsprachige Touristen beworben werden soll (Beispiel 9).

Sammelt in der Klasse. Was zeigt ihr in einem Video über eure Stadt?

Beispiel 8: Video über eure Stadt (aus: Jin/Rohrmann 2016: 34)

Projekt: Wählt eine Sehenswürdigkeit bei euch aus und macht dafür ein Werbeplakat oder eine Werbepräsentation für deutschsprachige Touristen.

Beispiel 9: Werbeplakat (aus: Jin/Rohrmann 2015: 58)

Eine Variante, die eigene Stadterkundungen mit sprachlichen Lernzielen verknüpft, bietet Prima Plus in Band A1.2 an (vgl. Abbildung 42).

Auch Beste Freunde (A1.2 und A2.2) greift Varianten der eigenen Stadterkundung auf, im Beispiel aus Band A2.2 dann stärker mit Fokus auf die Auseinandersetzung mit der eigenen Umgebung aus persönlicher Perspektive, und weniger mit touristisch-werbendem Blick (vgl. Abbildung 43 und Abbildung 44).

Team Deutsch 3 (B1) schlägt nicht nur eine Stadtführung der eigenen Umgebung für eine deutschsprachige Gruppe vor, sondern bezieht weitere The- 


\section{Projekt}

\section{Das ist mein Land!}

1a Macht ein Plakat über euch und euer Land. Zeichnet eine Karte und tragt einige Städte ein. Ihr könnt auch die deutschen Namen für die Städte dazuschreiben.

b Zeichnet oder klebt Bilder mit typischen Dingen auf die Karte.

2a Schreibt kurze Texte über euch. Antwortet auf die Fragen.

- Wie heißt du?

- Woher kommst du?

- Wo wohnst du?

- Was machst du gern?

- Was ist typisch für dein Land oder für deine Stadt?

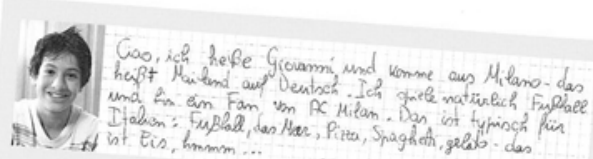

Schneidet die Texte aus und klebt sie zusammen mit einem Foto auf die Karte.

Präsentiert euer Plakat.

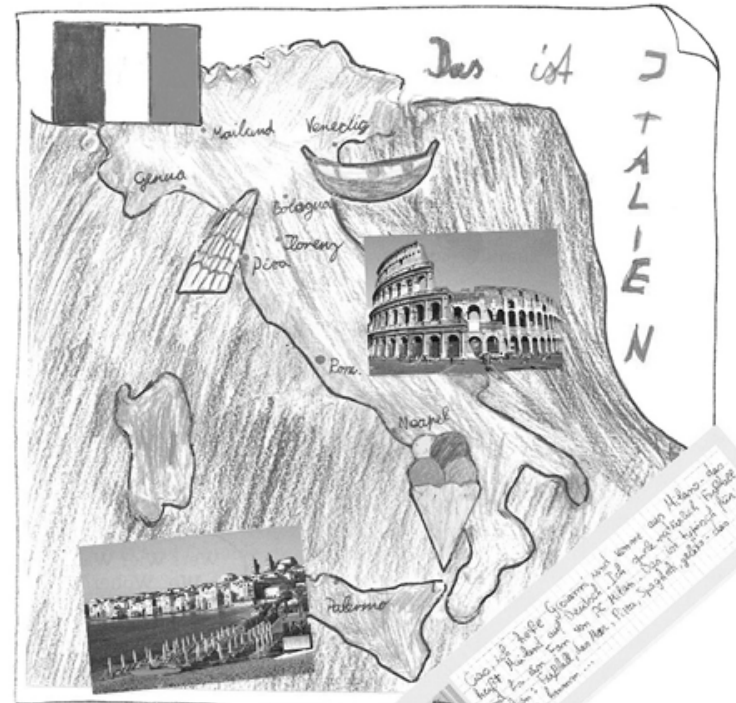

Abbildung 41: Das ist mein Land! (aus: Georgiakaki 2013: 25) 
Blick aus dem Fenster zu Hause / in der Schule

a Was siehst du?

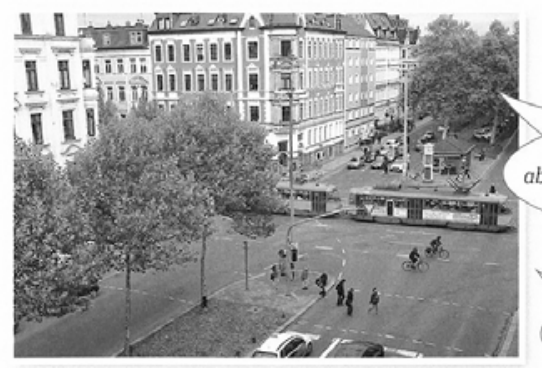

es gibt + Akkusativ

In Frankfurt gibt es einen

Fluss.

Bei uns in ... gibt es einen Fluss, aber man kann ihn von hier aus dem

Fenster nicht sehen.

Es gibt einige Autos.

Manchmal fährt eine Straßen-

Die Häuer sind alt.

rats. ther sind alt.

b Mach eine Präsentation zu deiner Stadt oder deinem Dorf.
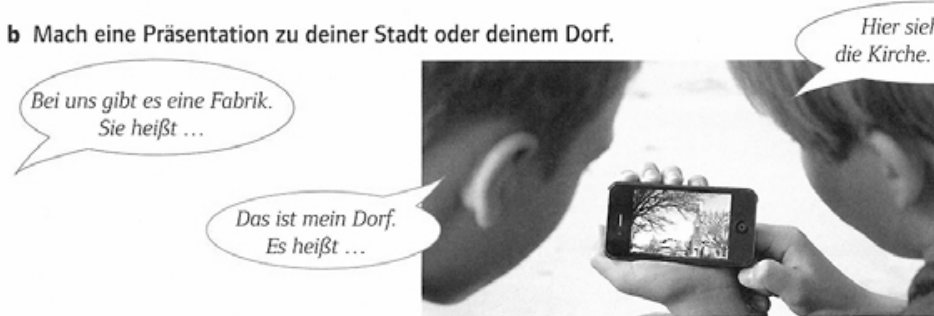

Abbildung 42: Blick aus dem Fenster (aus: Jin/Rohrmann 2014: 52) 


\section{Projekt}

das Kino: Filme anschauen, der Park: ?

der Stadtplatz: interessant für die Besucher? Was kann man dort machen? Sammelt Ideen und macht Notizen.

(2) Plant einen Spaziergang durch eure Stadt für eure Gäste.

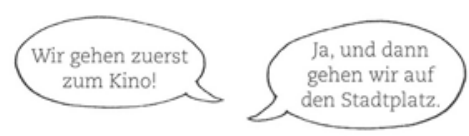

Macht nun ein Plakat mit Bildern und Texten.

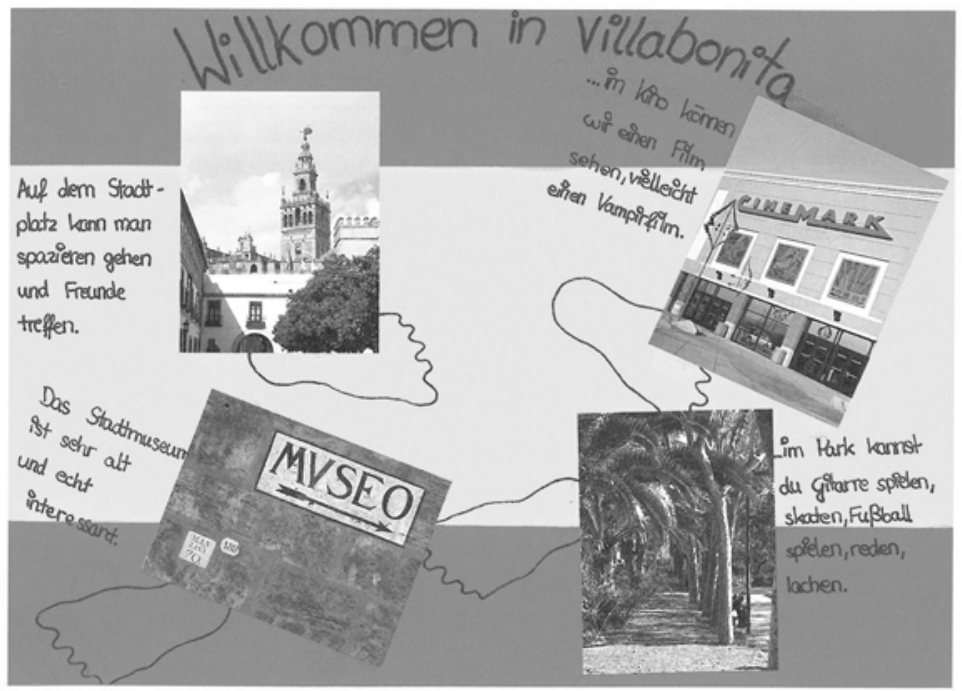

4a Präsentiert euren Spaziergang in der Klasse.

- Das ist unser Spaziergang durch ...

- Das ist der ...platz. Hier gibt es ...

" Im ...park könnt ihr ...

b Welcher Spaziergang gefällt euch am besten?

Abbildung 43: Das ist unsere Stadt: Herzlich willkommen (aus: Georgiakaki et al. 2014: 39) 


\section{Das ist unser Lieblingsort!}

1) Habt ihr einen Lieblingsort in der Stadt? Sammelt Ideen und macht Notizen.

- Was kann man dort alles machen?

-Was gefällt euch dort?

-Wie fühlt ihr euch dort?

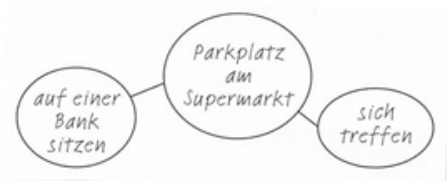

2a Macht Fotos von eurem Lieblingsort.

Ihr könnt auch einen kleinen Film mit dem Handy drehen.

b Wählt die besten Fotos aus und schreibt einen Text über euren Ort.

C Stellt eure Fotos und Texte auf einem Plakat zusammen.

\section{Unser Lieblingsort}

In der Stadt ist ein grober Parkplatz. Er gehört zu einem Supermarkt und ist sicher nicht besonders schön. Aber wir finden inn super. Wir treffen uns manchmal nach der Schule dort. Dann reden wir, erzählen von nervigen Lehrern oder doofen Prüfungen, holen uns eine Limo oder Cola im Supermarkt und manchmal eine Packung Chips. Hinter dem Parkplatz gibt es einen kleinen Park. Leute gehen wit ihren Hunden dort spazieren, man kann auf einer Bank sitzen, miteinander reden und ein bisschen Unsinn machen.

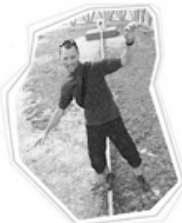

Einmal hat jemand eine Slackline zwischen zwei Bäumen aufgehängt, das war sehr lustig! Am Sonntag gibt es immer einen Flohmarkt auf unserem Parkplatz. Einmal haben wir auch schon selbst Sachen verkauft, Kleider, Bücher, CDs und Spiele.

Wir finden unseren Parkplatz sehr schön. Nur die skateboarder wünschen sich, dass sie hier Skateboard fahren und trainieren dürfen. Aber das will der supermarkt nicht und das können wir sogar verstehen. ;-)

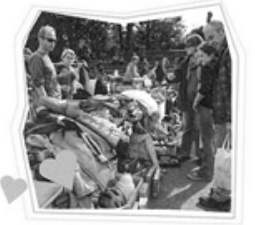

3 Präsentiert euer Plakat oder den Film in der Klasse. Welcher Lieblingsort gefällt euch am besten?

Abbildung 44: Das ist unser Lieblingsort (aus: Georgiakaki et al. 2015: 21) 
men ein, die für deutschsprachige Austauschschüler oder Freunde aufbereitet werden sollen, u. a. die Frage danach, welche Souvenirs sich aus dem eigenen Ort oder Land gut vermarkten lassen könnten und weitere Dokumentationsaufgaben mit Bezug zur Ausgangskultur. Im Sinne der Kontaktdidaktik wird hier das eigene Umfeld der Lernenden für Vertreter der Zielsprachenkulturen erarbeitet, dabei wird - durch den Blick für Fremde - Reflexion auch über das eigene Lebensumfeld angeregt und verhindert, dass Deutschlernende zu potentiellen Touristen im deutschsprachigen Raum reduziert werden.

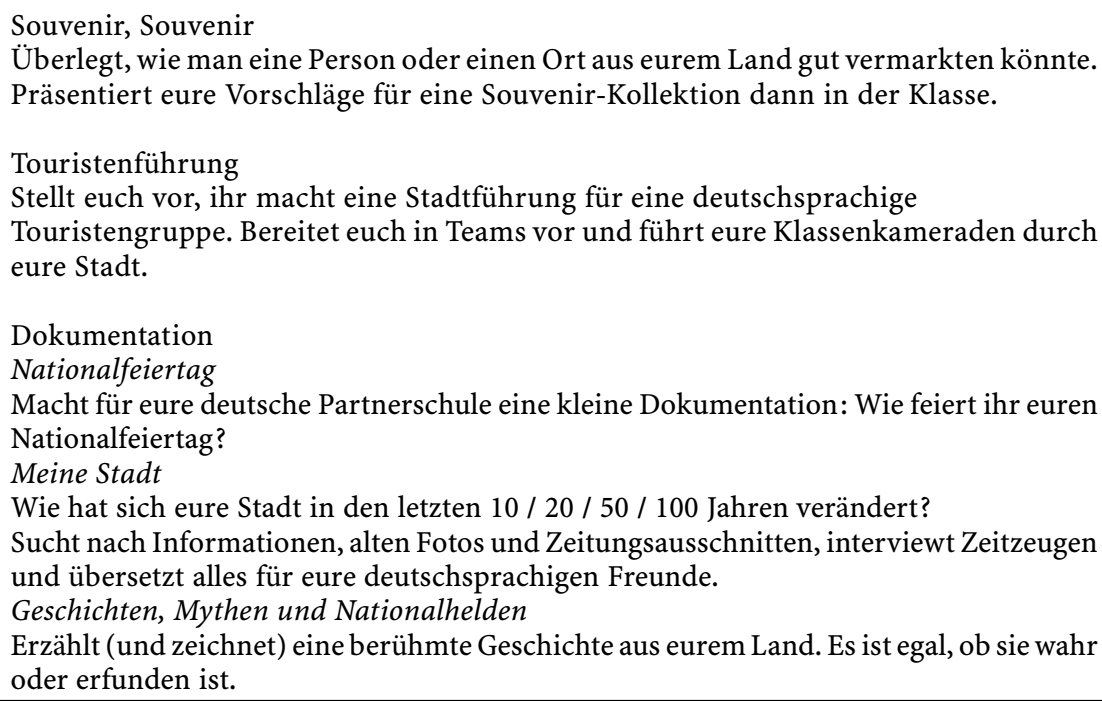

Beispiel 10: Souvenir, Souvenir; Touristenführung; Dokumentation (aus: Esterl et al. 2016: 80)

Eine weitere Variante der Erkundung der eigenen Umgebung zeigt die folgende Aufgabenreihenfolge aus Team Deutsch 3 (B1). Sie lässt Schüler zunächst über Sprachen in ihrem eigenen Land reflektieren und schließt so gut an Ziele eines kulturreflexiven Lernprozesses an. Anschließend gehen die Schüler auf Spurensuche nach "Deutsch " im eigenen Land und denken über eigene "Lieblingswörter im Deutschen « nach. In der Aufgabe werden so in reflektierter Weise Verbindungen und bewusste oder unbewusste Bezüge von Lernenden zur Zielsprache Deutsch (sowie anderen Sprachen) sichtbar sowie nutzbar gemacht (vgl. Abbildung 45).

Eine andere Form, persönliche Bezüge auf sprachlicher Ebene - nicht nur zur Zielsprache - herzustellen, zeigt Dimensionen. Magazin 3 (B1). Hier werden die Lernenden im Sinne einer Mehrsprachigkeitsdidaktik gebeten, Sprachenportraits anzufertigen und somit die Zielsprache Deutsch in Bezug zu setzen zu ihrem gesamten plurilingualen Repertoire (vgl. Abbildung 46). Durch die kur- 


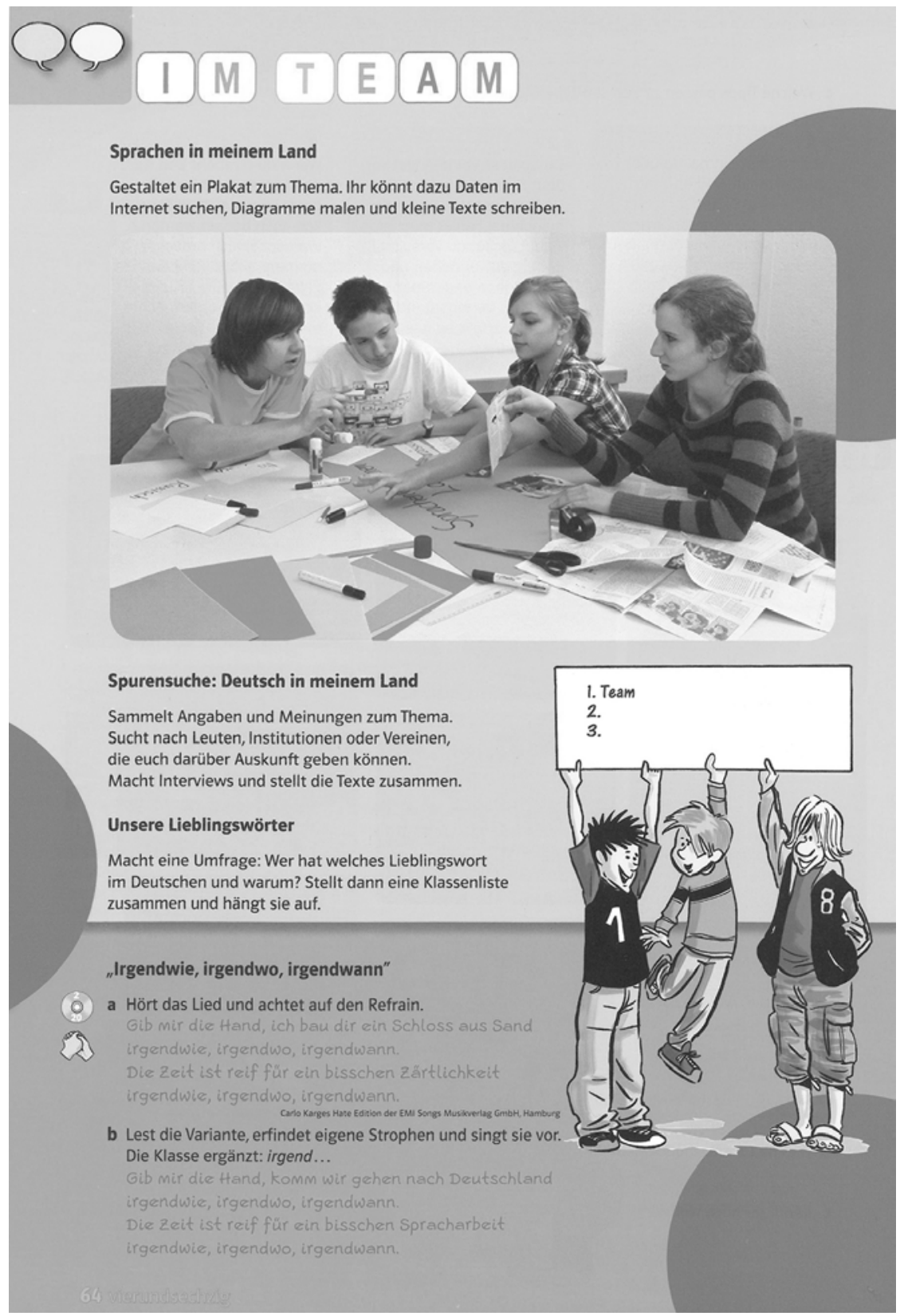

Abbildung 45: Sprachen in meinem Land; Spurensuche; Lieblingswörter (aus: Esterl et al. 2016: 64) 
zen zu schreibenden Texte in Kombination mit Zeichnungen werden ihnen auch affektive Zugänge zum Thema ermöglicht.

Bezüge zur eigenen Lebenswelt mit Verbindungen zum Deutschlernen können auch über eigene Interessen hergestellt werden. So findet sich in Fantastisch A1 der Vorschlag, dass eigene Lieblingsbuch zu präsentieren, wobei ausdrücklich betont wird, dass das Buch nicht auf Deutsch sein muss. Anstelle das Buch für deutschsprachige Schüler zu präsentieren, die vielleicht die eigene Mutteroder Ausgangssprache lernen, z. B. im Rahmen von Austauschpartnerschaften, wird in der Aufgabenreihenfolge vorgeschlagen, das Buch einem Mitschüler zu empfehlen. Dies dürfte insofern vertretbar sein, als dass die Aufgabe so real durchgeführt werden kann und - sollte kein Austausch bestehen - nicht nur für fiktive Personen geschrieben wird.

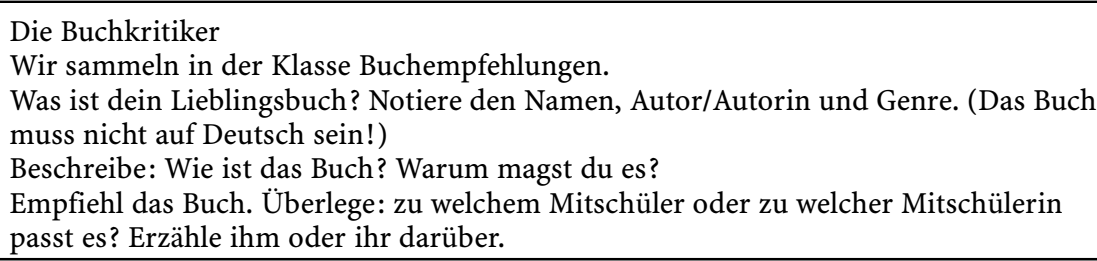

Beispiel 11: Die Buchkritiker (aus: Maccarini et al. 2018: 98)

Zum Thema "Schule international« findet sich eine ähnliche Aufgabenstellung in Fantastisch A1, die durch den Einbezug der eigenen Lernumgebung persönliche Bezüge der Lernenden zum Deutsch lernen aktivieren kann.

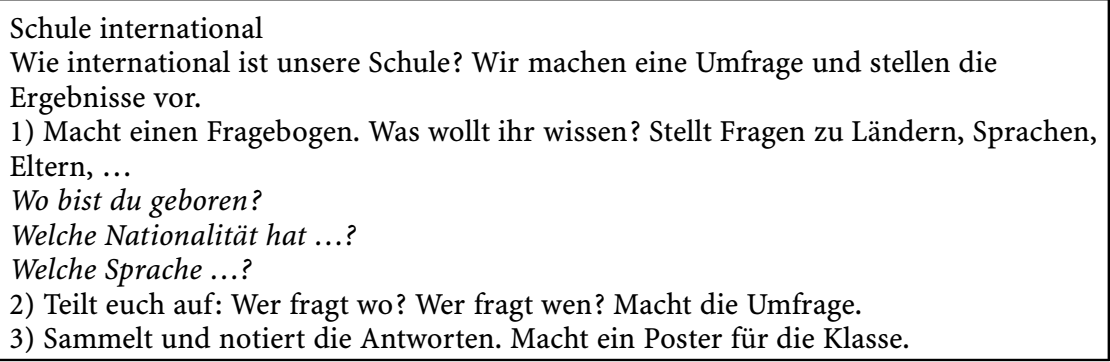

Beispiel 12: Die Schule international (aus: Maccarini et al. 2018: 112)

Eine andere Form Aktivitäten, die Bezug zur eigenen Lebensumgebung herstellen kann, zeigt das folgende Aufgabenbeispiel aus Klasse! A1 zum Thema Nahrungsmittel. Essen und Trinken mal anders, nämlich nicht mit den häufig abgebildeten, typisch-traditionellen Gerichten aus deutschsprachigen Ländern, sondern mit Informationen zum Obstverzehr der Deutschen (vgl. Abbildung 47). Die anschließend zu erstellende Hitliste $\mathrm{zu} » O b s t$ für euer Land oder eure Klasse« ermöglicht es den Lernenden, Bezüge zur eigenen Lebensrealität 


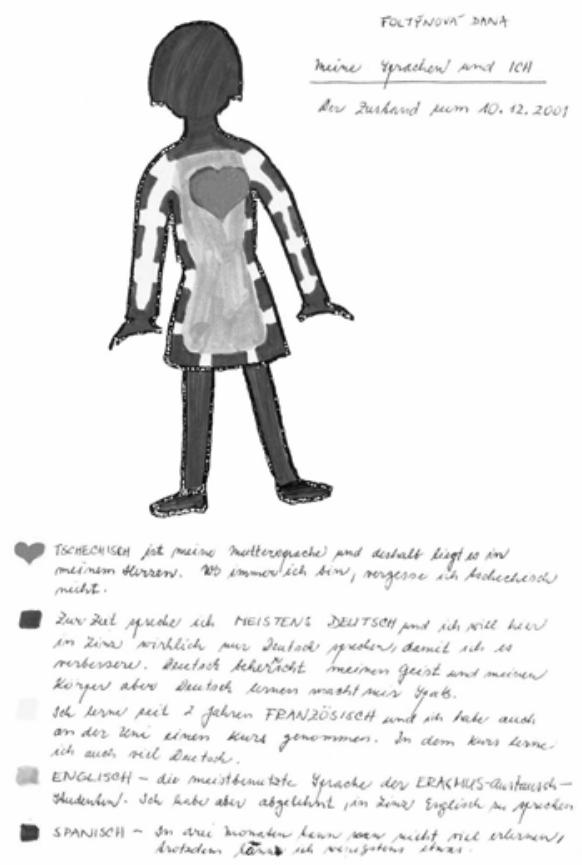

MARINA

Russisch ist in munem Horz. Das ist meine Nuthersprache. Toh lide Rusrich Estrisch-meine Hainde. Estrusath Brauche ich wie die Hande. Jch wohne in extland und ich mu $\beta$ diese Bontake konner. Estrisch is grin weil un Estond schóne listur Yrun weib in clest. Das ist meine Leeblingsprache. las int meine weil becu int fir lie ist blaw, weit becu ioh hoff, much Traumsprache und lon hope,

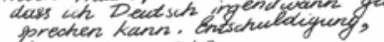
hier gobt es Fehler.

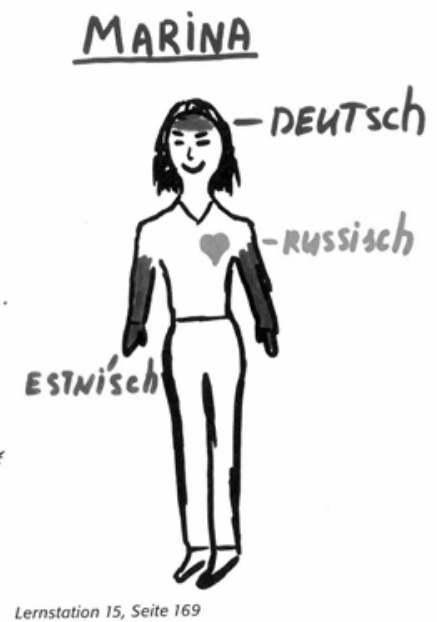

72 Lernstation 15 Die Welt der Sprachen - Die Sprachen der Welt Lernstation 15, Seite 169

Abbildung 46a: Sprachenportraits (aus: Jenkins et al. 2006: 72) 

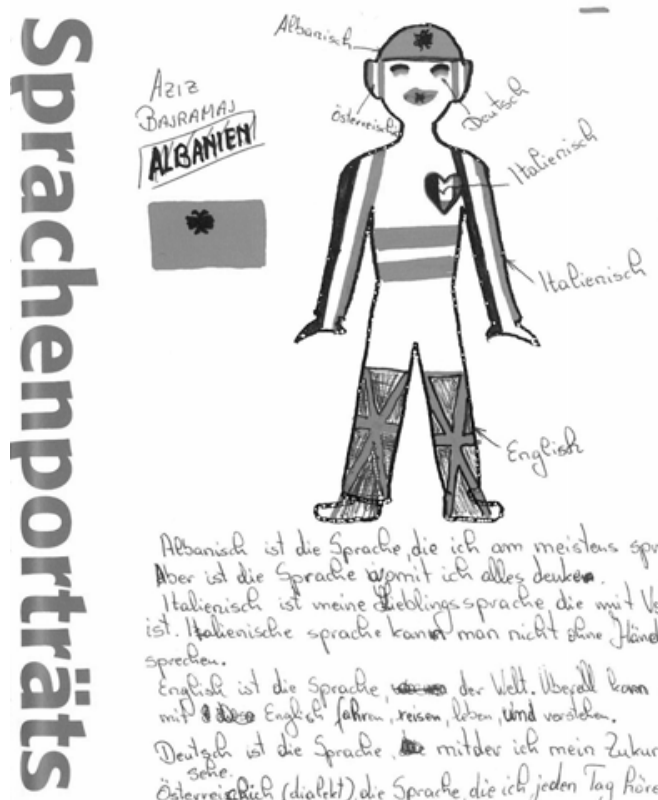

Albanish ist die Sprache die ich am meistens spreche.

Aber ist die Spracke bomit ich alles denke.

Italienisch ist meine Deblings sprache die mit Vibleliebe

ist. Itakenishe sprache kann man nicht ahne flande

sprechen. is ist die Sprache der Welt. Uberall kom man

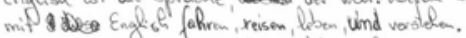

Deutsgh ist die Sprache mitder ich mein Zukunft

Österveischich (dialelt), die Sprache, die ich jeden Tag höre.

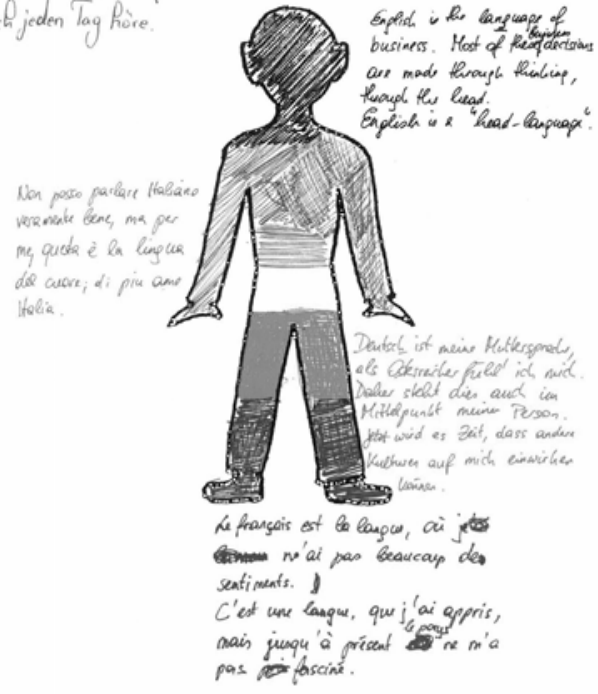

Die Welt der Sprachen - Die Sprachen der Welt Lernstation 15

Abbildung 46b: Sprachenportraits (aus: Jenkins et al. 2006: 73) 


\section{Landeskunde}

11 a Obst in Deutschland - Was essen die Deutschen am liebsten? Wie ist die Hitliste? Ratet und vergleicht in der Klasse. Hört dann die Lösung.

178 Im Durchschnitt essen die Deutschen 62,5 Kilo Obst im Jahr. Hier sind die Plätze 1 bis 5 .

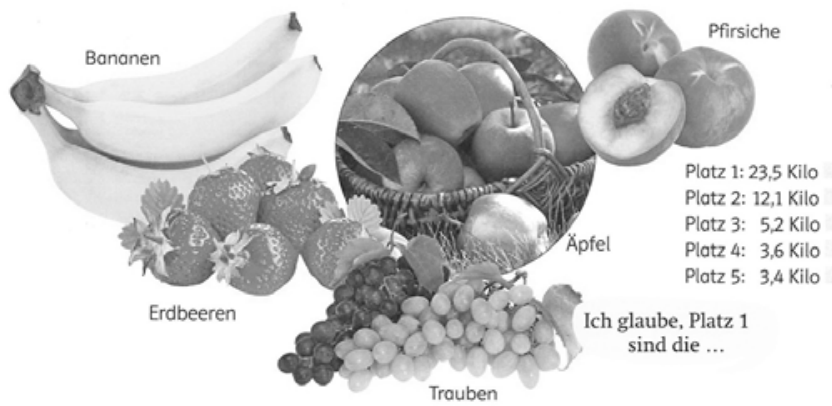

b Macht eine Hitliste „Obst “ für euer Land oder eure Klasse. Esst ihr gerne Obst? Was ist euer Lieblingsobst? Sprecht in der Klasse und erstellt eine Statistik.

$$
\text { Ich mag (keine) ... B Bei uns essen die }
$$

$$
\text { Leute oft ... Ich esse jeden Tag ... }
$$

a DAS essen die Deutschen?! - Lest die Meldung, seht die Fotos an und lest die Dialoge. Wie heißen die Lebensmittel? Die Dialoge helfen.

$$
\text { +++ Aktuell +++ Aktuell +++ Aktuell +++ Aktuell +++ }
$$

\section{Die Deutschen sind „Kannibalen“. Sie essen Amerikaner, Berliner,} Wiener und Hamburger.
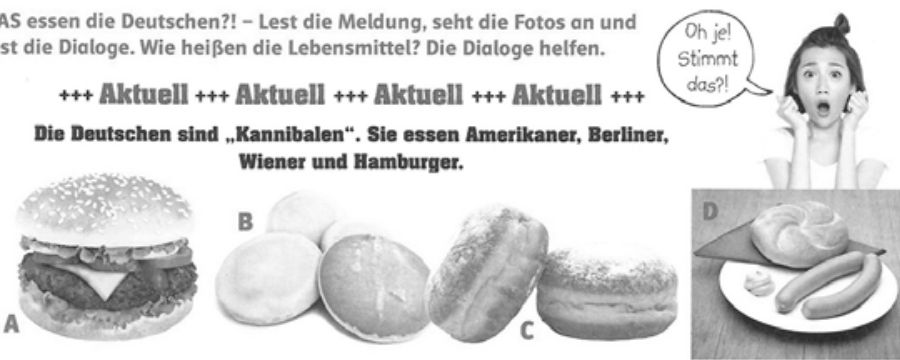

$1 \mid \begin{aligned} & \text { Nimmst du auch einen Hamburger? } \\ & \text { O Ja, mit viel Salat, Tomaten und Ketchup, bitte }\end{aligned}$

2 Möchtest du einen Berliner?

2 O Hm, ja, gerne. Mit Marmelade, lecker!

$3 \mid \begin{aligned} & \text { Was ist das? } \\ & \text { O Das sind zwei Wiener mit Brötchen. } \\ & \text { Wiener? Ist das Fleisch? } \\ & \text { O Ja, das sind Würstchen. }\end{aligned}$

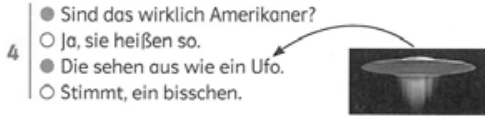

b Sprecht über die Speisen. Was ist süß? Was ist salzig? Was wollt ihr gern essen? Was nicht?

Abbildung 47: Landeskunde: Obst in Deutschland (aus: Fleer et al. 2018: 65) 
herzustellen und lässt sich leicht auf andere Themenbereiche übertragen. Auch wenn es sich auf den ersten Blick lediglich um einen Vergleich zwischen Zielund Herkunftssprachenland handelt, ist die Aufgabenstellung so konzipiert, dass persönliche Bezüge und kein Fakten gegenüberstellender Vergleich im Mittelpunkt stehen.

\subsection{Kulturreflexivität und Diskursivität}

Eine Reihe Beispiele für Aufgabenimpulse, die Diskurs anregen können, finden sich naheliegenderweise im Band Mitreden. Diskursive Landeskunde für Deutsch als Fremd- und Zweitsprache. Beispiele hierfür wären die Arbeitsblätter zum Thema »Schilderwelten« oder zum Thema »Karten, Karten, Karten« (vgl. Abbildung 48 und 49).

Prima Plus stellt in Band A2.2 in der Lektion "Austausch" Berichte von Austauschschülern, die einige Zeit im deutschsprachigen Raum verbringen, vor. Benannt werden sowohl positive Erfahrungen als auch Aspekte, die schwierig oder aus der Sicht der Austauschschüler ungewöhnlich sind (vgl. Abbildung 50). Dies kann zu einem relativierenden Bild des Zielsprachenlandes beitragen, gleichzeitig aber auch einen ersten Schritt zu einer Reflexion darüber, was für die Lernenden selbst selbstverständlich und was ungewöhnlich ist, bedeuten; vorausgesetzt dies wird im Unterricht aufgegriffen.

Auch Dimensionen. Magazin 3 (B1) eröffnet den "Fremden Blick von außen" und lädt zu Reflexion und Relativierung von Wahrgenommenen ein, indem Aussagen von Wissenschaftlern und Wissenschaftlerinnen aus aller Welt präsentiert werden, die berichten, was ihnen an Deutschen aufgefallen ist (vgl. Beispiel 13). 


\section{Schilderwelten}

1a. Sehen Sie sich die alte und die neue Version des Straßenschildes, Fußgängerüberweg' an. Was ist gleich, was ist unterschiedlich?
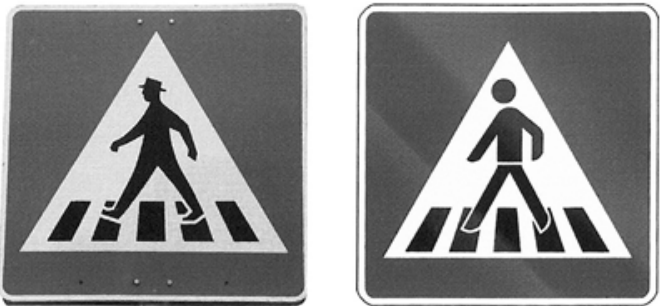

1b. Sehen Sie sich nun das aktuelle Schild ,Fußgängerzone' an. Was sehen Sie? Was ist anders? Sammeln Sie Merkmale und diskutieren Sie.

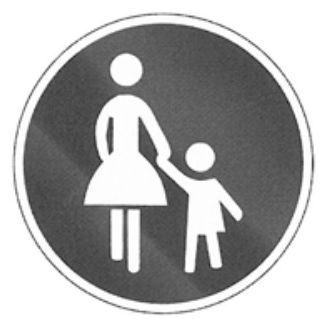

1c. Sie sind in einer Kommission, die ein neues Schild ,Fußgängerzone' entwerfen soll. Wie könnte so ein neues Schild aussehen? Warum? Zeichnen Sie und vergleichen Sie Ihre Schilder im Kurs.

Abbildung 48: Schilderwelten (aus: Altmayer et al. 2018: 32 (KV8)) 


\section{Karten, Karten, Karten}

2a. Schauen Sie sich folgende "Karten" an. Was zeigen sie? Wie wird das dargestellt?

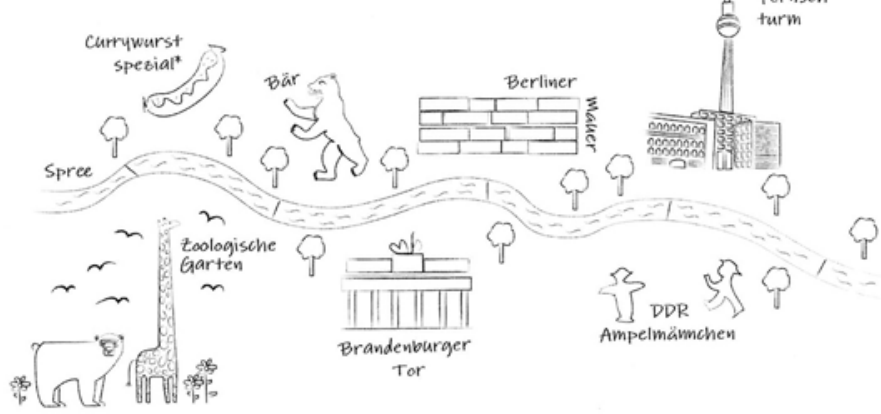

\section{Karte 1}

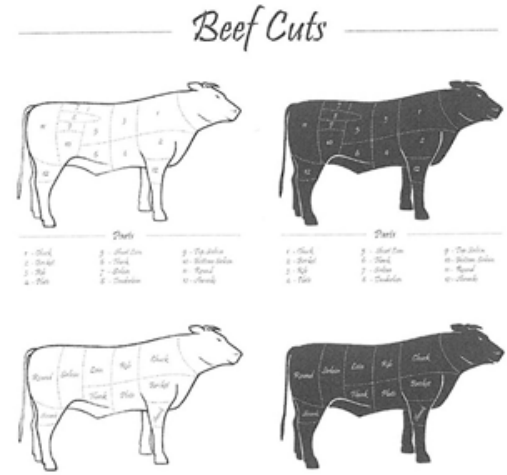

Karte 2

2b. Erstellen Sie in der Gruppe eine Karte zum Thema Mobilität. Bestimmen Sie zusammen, was Sie darstellen wollen. Und entscheiden Sie sich für eine Form der Darstellung auf der Grundlage der bisher diskutierten Karten.

2c. Galerie-Rundgang: Bitten Sie die Besucher, Ihre Karte zu beschreiben und diskutieren Sie mit ihnen.

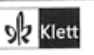

Abbildung 49: Karten, Karten, Karten (aus: Altmayer et al. 2018: 100 (KV50)) 
Austauschberichte

a Pedro, Maria und Luis sind Austauschschüler aus Santa Cruz de la Sierra. Nach der Rückkehr aus Deutschland müssen sie einen Bericht schreiben. In ihren Tagebüchern haben sie Notizen für ihren Bericht gemacht. Lies die Textteile. Was passt zusammen?

Heute war ein normaler Tag und ich bin zum ersten Mal in die Schule gegangen. Die ersten zwei Stunden (Deutsch) waren schrecklich Ich habe nicht viel verstanden und konnte mit niemandem sprechen. Aber später in der Pause war es besser und ich konnte einige Schüler kennenlernen. lem war ich allein mit dem Bus nach Hause fahren musste.

Gestern sind wir zum Dreiländereck gefahren (Belgien, Deutschland und Holland). Es war wunderschön. Wir sind auf einen Fernsehturm gestiegen.

Die Tage werden immer dunkler. Alles ist nur grau, nicht lebendig. Ich bin joggen gegangen und um 5 Uhr nachmittags war es schon total dunkel! Von November bis Februar leben die Deutschen bei Dunkelheit. Ich vermisse die Sonne von Santa Cruz.
Ich möchte so gerne für eine Woche zurückfahren, mit meiner Familie zusammen sein und in der Sonne Fußball spielen. Aber das kann ich nur trä̉umen! Luis

Ich hatte keine Ahnung, wo die Haltestelle war und musste jemanden fragen. Ich war total nervös und hatte Angst, dass ich im falschen Bus war. Alle haben mich komisch angesehen. Aber es war der richtige Bus! Pedro

Es war komisch, weil man gleichzeitig in drei Ländern war. Am letzten Wochenende waren wir dann Ski fahren in der Skihalle mit Gerd, Robert und Alexander. Das war super. Erst habe ich viel auf dem Boden gelegen, aber dann konnte ich zusammen mit den anderen fahren.

Maria

b Welche Überschrift passt zu welchem Tagebucheintrag?

\section{Geschafft! \\ Die besten Momente! \\ Heimweh!}

c Positive Erfahrungen und Probleme. Sammelt und macht einen Tabelle.

\begin{tabular}{l|l} 
Positive Enfahrungen & Probleme \\
\hline $\begin{array}{l}\text { bei den ersten Kontakten } \\
\text { mit Klassenkameraden }\end{array}$ & in den ersten Deutschstunden
\end{tabular}

Abbildung 50: Austauschberichte (aus: Jin/Rohrmann 2015: 27) 


\begin{abstract}
Gesundheit
Um es nett zu formulieren: Die Deutschen sind sehr gesundheitsbewusst. In keinem anderen zivilisierten Land ist das Phänomen "Kur» so verbreitet wie in Deutschland, ebenso die »Kreislaufstörung«. Und nirgendwo sonst unterhält man sich so gern über seine Befindlichkeit. Die Folge ist ein hervorragendes Gesundheitswesen. Qualität und Status des medizinischen Personals sind unbestreitbar. Zudem hat kein Land in Europa, mit Ausnahme Frankreichs, so viele gut sortierte Apotheken. Der hohe Arzneimittelverbrauch und der einfache Zugang zu medizinischer Versorgung führen zu den höchsten Pro-Kopf-Ausgaben im Gesundheitswesen der OECD-Länder nach der Schweiz, Norwegen und den USA. Entsprechend aufmerksam achtet der Deutsche auf sein Wohl.

(Lily Sprangers, Geschäftsführende Direktorin am Duitsland-Institut Amsterdam)

\section{Flohmarkt}

Ich habe mich oft gewundert, warum reiche Deutsche auf den Flohmarkt gehen. Dort sah ich, dass Leute gebrauchte Sachen für ein paar Euro verkaufen, während in der Nähe ihres Standes ein Volvo parkte. Für uns Indonesier ist ein Volvo ein Minister-Auto. Der Flohmarkt heißt bei uns „pasar miskin«, Markt der Armen. Er heißt so, weil die verkauften Waren abgenutzt sind oder Diebesgut. Geht jemand dorthin, wird er als arm stigmatisiert. Ein Besuch dort kann den sozialen Status verringern.

(Aziz Damsar, Soziologe, Indonesien)
\end{abstract}

Beispiel 13: Der fremde Blick von außen (aus: Jenkins et al. 2006: 106-107)

Unter dem Titel "Sprachen im DACH-Alltag« sollen in Band 3 von Team Deutsch (B1) auf einer Collage verschiedene Sprachen aus Prospekten, Anzeigen oder ggf. dem urbanen Raum identifiziert werden, inkl. der Bereiche, in denen diese Sprachen im deutschsprachigen Raum genutzt werden (vgl. Abbildung 51). Während eine explizite Thematisierung von Sprachenvielfalt in den einzelnen DACH-Ländern - wohl auch berechtigterweise - ausbleibt, regt die anschließende Aufgabe »Welche Sprachen spielen in eurem Alltag eine Rolle. Sammelt Beispiele.« $\mathrm{zu}$ einem Vergleich mit dem Zielsprachengebiet an und somit auch zu einer Auseinandersetzung damit, welche Sprachen wo frequent ssichtbar sind. Zwangsläufig dürften auf diese Weise Gründe hierfür zu hinterfragen sein. Gleichzeitig erlaubt diese Aufgabensequenz auch - im Sinne der Kontaktdidaktik - Bezüge zwischen vermeintlicher Ziel- und Ausgangskultur im eigenen Lebensraum herzustellen, so dass die vermeintlichen Zielsprachenkulturen nicht nur thematisiert und kontrastiert, sondern auch diskutiert werden können.

Zwischen den Kulturen bietet zahlreiche Arbeitsblätter, die unterschiedliche Verhaltensnormen und Gewohnheiten zur Diskussion stellen und somit darüber reflektieren lassen, was für den einen üblich, den anderen aber unüblich ist. Die folgenden Aufgabenbeispiele (vgl. Abbildung 52 und 53) zeigen solche Aktivitäten für die Themen `Höflichkeit: Begrüßungen`sowie `Tabuthemen`. In beiden Fällen geht es nicht um angeblich im Zielsprachengebiet angemessenes Verhalten, sondern um eine eigene Einschätzung, was für einen selbst zutrifft und dann ggf. Erwartungen, was in den DACH-Ländern üblich sein könnte. 


\section{LEE KIIION $)^{-1}$}

\section{Meine Sprachenwelt}

\section{1) Sprachen im DACH-Alltag}

a Seht euch die Collage an. Findet heraus, welche Sprachen in welchen Bereichen vorkommen.

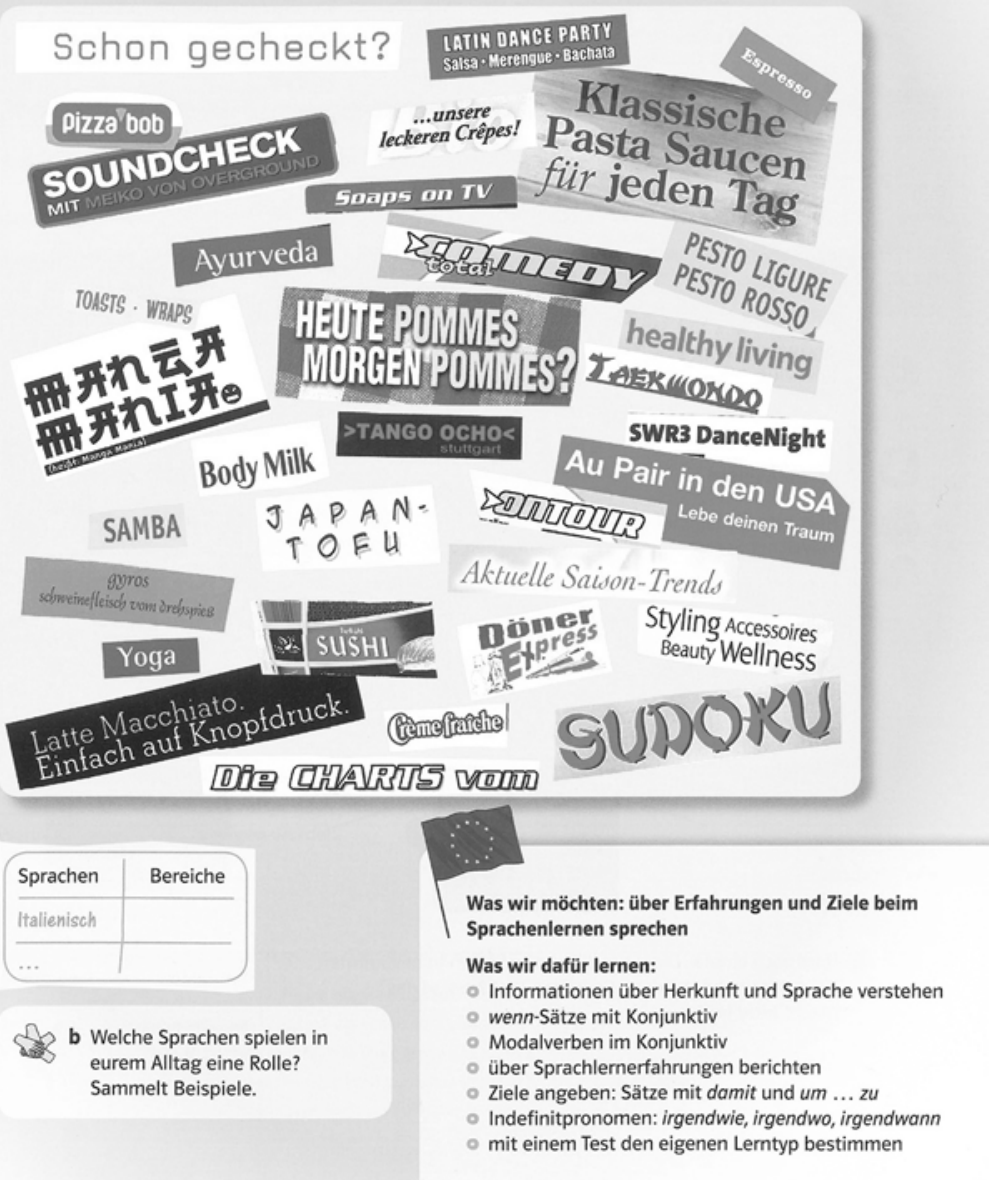

Abbildung 51: Sprachen im DACH-Alltag (aus: Esterl et al. 2016: 57) 


\section{Kïß die Hand}

Lesen Sie folgende Aussagen und beantworten Sie in der Gruppe folgende Fragen.

Tragen Sie ein:

- Welche Verhaltensweisen finden Sie angenehm, positiv?

- Welche mögen Sie nicht?

- Welche sind thnen fremd?

- Welche sind threr Meinung nach in Deutschland, Österreich oder der Schweiz üblich?

1. Zu jeder richtigen Begrüßung gehört ein Handschlag/Händedruck.

2. Der Händedruck muß fest sein.

3. Es gibt Begrüßungsformulierungen mit religiöser Bedeutung.

4. Nur sozial Gleichgestellte geben sich die Hand

5. Mănner, die sich z.B. aus Kneipen, Sportvereinen und ähnlichem kennen, kłopfen sich oft auf die Schulter.

6. Die Menschen vermeiden jeden Körperkontakt.

7. Gute Bekannte oder Freunde begrüßen sich auch mit einem $\mathrm{Ku} ß$ oder einer $\mathrm{Um}$ armung.

8. Nach der ersten Bekanntmachung oder Begrüßung ist es höflich, sich nach Beruf, Ausbildung, Kinderzahl und Einkommen zu erkundigen.

9. Beim Betreten von kleineren Läden, öffentlichen Büros und ähnlichem sagt man einen kurzen Gruß und verabschiedet sich auch.

10. Der Mann gibt der Frau einen Handkuß bei Begrüßung und Abschied.

11. Sozial Niedrigstehende begrüßen sozial Hōherstehende zuerst.

12. Eine lange Begrüßung und viele Fragen nach dem Wohlbefinden sind eine gute Voraussetzung für ein geschäftliches Gespräch.

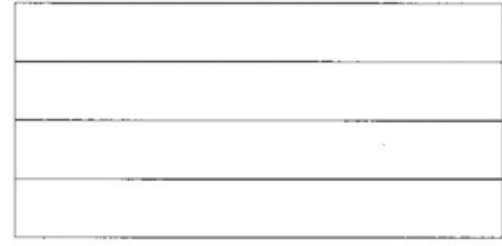

13. Beim Betreten eines Zugabteils oder einer Arztpraxis ist es üblich, jeden einzelnen zu begrüßen.

14. Zur Begrüßung reicht es, die Augenbrauen hochzuziehen und zu lächein.

15. Es ist höflich, daß man sich bei der Begrüßung direkt in die Augen sieht.

16. Es ist höflich, Angestellte/Beamte im Amt bei der ersten Begegnung zu fragen: „Wie geht es Ihnen?"

17. Hundebesitzer freuen sich immer sehr, wenn man auch ihren Hund begrüßt.

18. Zur Begrüßung gehört, daß man den Hut abnimmt.

19. Eine sitzende Person steht zur BegrüBung auf.

20. Die Nennung des Titels oder des Berufs gehört unbedingt zur Begrüßung, z.B. "Guten Morgen, Herr Doktor.“, „Guten Tag, Frau Kommerzienrat."
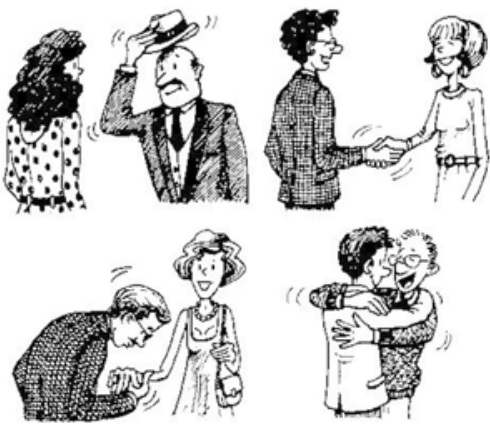

Abbildung 52: Küß die Hand (aus Hansen/Zuber 1996: 45) 


\section{„Wie geht's?}

- Lösen Sie die Aufgaben in Partnerarbeit.

- Entscheiden Sie gemeinsam, ob und wem gegenüber (in welcher Situation) die folgenden Fragen oder Bemerkungen in den deutschsprachigen Ländern angebracht sind. Tragen Sie jeweils den oder die passenden Buchstaben ein $(a-e)$.

- Vergleichen Sie anschließend ithre Antworten mit denen aus der Gesamtgruppe und diskutieren Sie Ihre Überiegungen untereinander.
a) Unbekannter
b) Chet/in
c) gute/r Bekannte/r
d) enge Freundin/enger Freund
e) nicht angebracht

\section{1. „Wie geht's?"}

2. "Darf ich mir mal den Stift leihen?"

3. "So etwas Blödes!"

4. „Wieso haben Sie keine Kinder?"

5. „Sind Sie religiős? Bist du religiös?"

6. "Gehen wir zusammen Kaffee trinken?"

7. "Woher kommen Sie gerade?"

8. „Wie alt sind Sie?"

9. „Oh, ich dachte, Sie sind älter."

10. „Sie sehen heute aber schlecht aus."

11. „Können Sie mir Geld leihen?“

12. "Liebst du mich?"

13. "Haben Sie eine Freundin / einen Freund?"

14. „Wieviel verdienen Sie im Monat?"

15. „Ich finde es hier furchtbar langweilig."

16. „Gestern hatte ich den ganzen Tag Magenschmerzen."

17. "Oh, dieser schreckliche Regen!"

18. "Das steht ihnen aber gut."

19. „Unsere Regierung ist wirklich das Letzte!

20. „Sind Sie verheiratet?“

21. „Welche Partei haben Sie / hast du gewählt?“
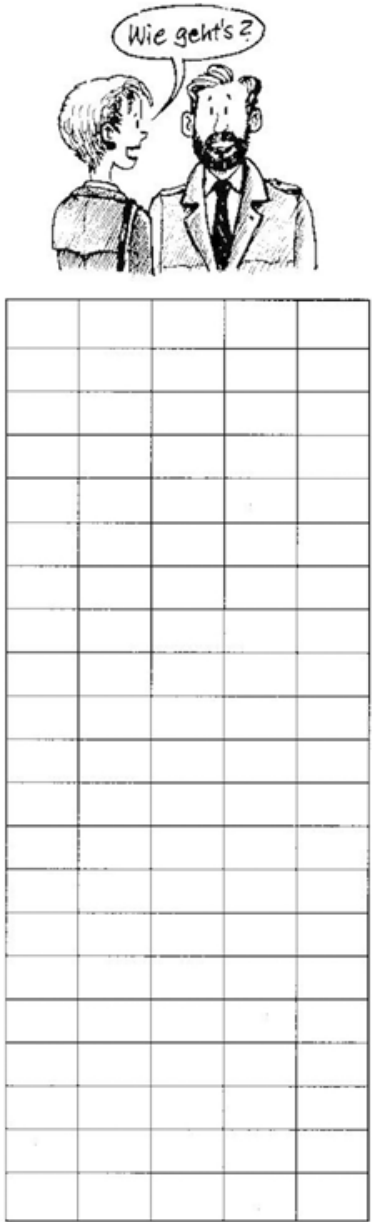

Abbildung 53: Wie geht's? (aus Hansen/Zuber 1996: 45) 
Obige Beispiele haben die eigene Situation als Startpunkt und sind mehr oder weniger zielsprachenunabhängig einsetzbar. Die Lernenden erhalten vorab keine Informationen über den Umgang mit dem jeweiligen Phänomen im Zielsprachenland, die vermeintlich fremde Kultur ist nicht - wie häufig insbesondere in kontrastiv angelegten Lehrwerken - Ausgangspunkt. Eine kritische Reflexion erfolgt erst durch den Vergleich in der Gruppe oder Klasse und mit Hilfe des Inputs der Lehrperson dann auch in Bezug zur Zielkultur; entsprechende Hintergrundinformationen zum Umgang mit den einzelnen Themen in DACH bietet Zwischen den Kultur jeweils als Informationen für Lehrende bzw. Lösungsblätter an.

Dimensionen. Magazin 3 (B1) geht ähnlich vor, allerdings mit Fotocollagen. Gezeigt werden beispielsweise Kühlschrankinhalte (vgl. Abbildung 54) oder Frühstücksutensilien (vgl. Jenkins et al. 2006: 4-5), in diesem Fall zwar aus $\mathrm{DACH}$, ermöglicht wird den Lernenden aber auch hier, zunächst eigene Gewohnheiten als Ausgangspunkt zu nehmen, diese in der Gruppe zu vergleichen und reflektieren und erst abschließend in Bezug zu zielkulturellen Eigenarten zu setzen. 


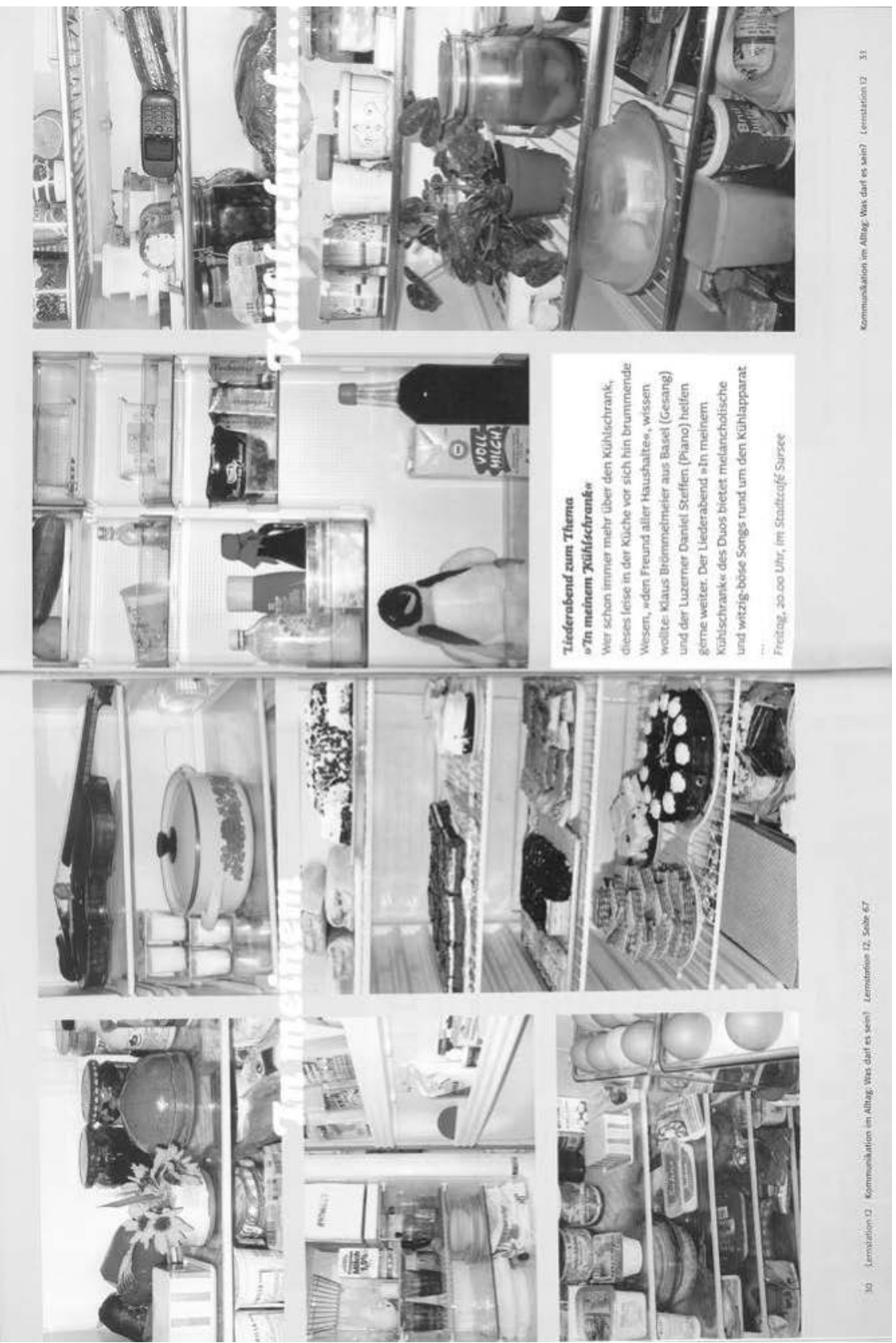

Abbildung 54: In meinem Kühlschrank (aus Jenkins et al. 2006: 30-31) 


\section{Ausblick}

Lehrwerke - digital oder als Print eingesetzt - spielen nach wie vor im schulischen Alltag eine unterrichtssteuernde Rolle. Die große Nachfrage wird u.a. sichtbar an der Anzahl der jährlich neu herausgegebenen Lehrwerke und zusätzlichen Lehr- und Lernmaterialien. Die gegenwärtige fachdidaktische Diskussion um die Rolle von Lehrwerken für den und im Fremdsprachenunterricht konzentriert sich stärker auf die Frage eines optimalen Einsatzes im Unterricht als auf eine Grundsatzdebatte des Lehrens und Lernens mit oder ohne Lehrwerk (siehe Kapitel 2). Eine der größten Herausforderungen der Didaktik und im Unterrichtsalltag scheint es zu sein, in der nicht selten »aufgeblähten« »Materialschwemme" eine optimale Lehrwerksverwendung zu finden, oder mit anderen Worten: Was bieten das jeweils im Einsatz befindliche Lehrwerk und weitere, zur Verfügung stehende Zusatzmaterialien an und wie kann ich mit diesem Angebot optimal - entsprechend der jeweiligen Zielgruppe, Rahmenbedingungen und Lernzielen - unterrichten. Denn, es handelt sich um eine Wechselwirkung, bei der unterstrichen werden muss, dass sowohl die Unterrichtsgestaltung als auch die Lehrwerkgestaltung von unterschiedlichen externen und schulinternen Faktoren geprägt ist: überregionale curriculare Vorgaben wie der GER oder regionale Rahmenlehrpläne können die Lehrwerke genauso determinieren wie Schultyp und das Lernniveau der Lernenden. Der (curriculare) Kontext, in dem ein Lehrwerk erscheint, hat einen großen Einfluss auf die Inhalte und Schwerpunkte sowie die jeweils im Lehrwerk getroffenen didaktischen Entscheidungen, dies gilt ebenso für landeskundliche Inhalte. 30 Jahre nach Veröffentlichung der ABCD-Thesen dürfte die Landeskunde ein legitimer Teil der im Rahmen des fremdsprachlichen Unterrichts vermittelten Inhalte sein. Lehrwerkautoren bzw. Verlage treffen jedoch unterschiedliche Entscheidungen bezüglich landeskundlicher Inhalte, räumen der Landeskunde unterschiedlich viel Platz ein und entscheiden sich für unterschiedliche didaktische Konzepte und Ansätze, die für Landeskunde und deren Didaktik entwickelt wurden (siehe Kapitel 3). Es lässt sich in etwa eine Entwicklung von faktenorientiert, über handlungsorientiert zu kulturreflexiv feststellen, wobei heut- 
zutage eine so deutliche Trennung in aller Regel in Lehrwerken nicht mehr wieder $\mathrm{zu}$ finden ist und eher Mischformen verschiedener landeskundlicher Ansätze in Lehrwerken erscheinen.

Die Analysen in Kapitel 4 dieses Bandes zeigen, dass die Umsetzung der didaktischen Ansätze und Konzepte von Landeskunde in polnischen und niederländischen DaF-Lehrwerken nicht nur auf der Makroebene (von Land zu Land), sondern auch auf der Mesoebene (von Lehrwerk zu Lehrwerk) unterschiedlich ausfällt. Zum Teil unterschiedliche curriculare Vorgaben in Polen und den Niederlanden führen direkt oder indirekt $\mathrm{zu}$ anderen didaktischen Entscheidungen und Schwerpunkten bezüglich Landeskunde. Aber auch für die verschiedenen Lehrwerke aus einem der beiden Länder konnte festgestellt werden, dass sie in der Thematik und Didaktik der Landeskundevermittlung unterschiedlich vorgehen.

Die Lehrwerkanalyse ergab, dass die analysierten polnischen und niederländischen DaF-Lehrwerke inhaltlich viele Ähnlichkeiten aufweisen; die Lehrwerke spiegeln die kulturelle und sprachliche Vielfalt der DACH-Länder wider und bieten einen Einblick in die Alltagskultur. Dabei wurde festgestellt, dass jedes Lehrwerk unterschiedliche Konzepte und Ansätze zur Landeskunde anbietet und andere Schwerpunkte setzt. Die angebotenen landeskundlichen Inhalte passen zum Lektionsthema, wobei der GER indirekt seinen Einfluss gelten lässt. In den niederländischen Lehrwerken fällt auf, dass sie die sprachliche und kulturelle Nähe zum Zielsprachengebiet mittels kontrastiven Ansatzes nutzen. Für die polnischen Lehrwerke lässt sich festhalten, dass jedes Lehrwerk einem anderen Ansatz folgt: Kompass Team bleibt überwiegend faktenorientiert, Mit links! ist dem kommunikativen Ansatz verpflichtet und akzentuiert die kommunikative Handlungsfähigkeit in Alltagssituationen, Meine Deutschtour bietet Aufgaben, die beide Ansätze umsetzen, versucht vereinzelt aber auch kulturreflexive Haltung zu stimulieren.

Hinsichtlich der Präsentation bzw. Integration des landeskundlichen Angebots ist festzustellen, dass sowohl die polnischen als auch die niederländischen Lehrwerke implizit und explizit vorgehen. Dabei fällt auf, dass die polnischen Lehrwerke und teilweise auch das niederländische TrabiTour das explizite Angebot als additives Extra anbieten, während Neue Kontakte und Na klar! Landeskunde in die Lehrwerksprogression eingebunden haben, und zwar mehrheitlich in Lesetexten mit Textverständnisfragen. Dies dürfte auf den Kontext des niederländischen Fremdsprachenunterrichts zurückzuführen sein: die verbindlichen niederlandeweiten Abschlussprüfungen beinhalten $\mathrm{zu}$ mindestens 50 \% rezeptives Leseverständnis für das Prüfungsfach Deutsch.

Insgesamt kann auch festgehalten werden, dass das landeskundliche Angebot inhaltlich und konzeptuell adressatengerecht, d.h. passend zur Zielgruppe, gestaltet wird. 
Der Einfluss des Kontextes zeigt sich ebenfalls in der Themenauswahl, nämlich im Sinne der geografischen Nähe, die insbesondere die niederländischen Lehrwerke sausnutzen k können, indem sie nicht nur die bekanntesten Sehenswürdigkeiten im deutschsprachigen Raum, sondern auch kulturelle Phänomene, die sich beispielsweise in der Grenzregion finden, aufnehmen und behandeln, wie z. B. das Einkaufszentrum CentrO in Oberhausen im niederländischen Lehrwerk Neue Kontakte. Nähe spielt im niederländischen Kontext eine bedeutende Rolle; nicht nur in sprachlicher, sondern auch in kultureller Hinsicht sind sich die Nachbarländer oft ähnlich. Niederländische Lehrwerke tendieren dann oft dazu, gerade solche Themen, die in den Niederlanden und den DACH-Ländern auffällig anders sind, zu behandeln, wobei in der Auswahl eine niederländische Brille erkennbar ist. So gibt es einen Beitrag zum Mittagessen in der Schulkantine, was es in den Niederlanden nicht gibt, und werden deutlich anders als in den Niederlanden aussehende Phänomene im urbanen Raum, wie deutsche PKW-Nummernschilder und Straßenschilder thematisiert und abgebildet. Inhaltlich passen die vorgestellten Phänomene zum Thema des jeweiligen Kapitels.

In den polnischen Lehrwerken werden die landeskundlichen Kenntnisse systematisch - im Zusammenhang mit den thematisch-inhaltlichen Schwerpunkten der jeweiligen Lektion - angeboten. Dabei sind Prinzipien der Schülerund Kommunikationsorientierung ${ }^{27}$ klar erkennbar.

Der vorliegende Band hat gezeigt, wie entscheidend der Kontext in der Lehrwerkgestaltung ist, will aber gleichzeitig auch dafür sensibilisieren, dass Kontextbewusstsein und der Blick über den eigenen Kontext hinaus sehr aufschlussreich und inspirierend sein kann. Die angebotene Auswahl an Lehrwerkinhalten zur Vermittlung von landeskundlichen Informationen kann zur Reflexion anregen und sowohl praktizierende als auch angehende Lehrkräfte, Lehrwerkautoren und Verlage dazu inspirieren, die im eigenen Kontext bekannten und eingesetzten Lehrwerke aus einer anderen Perspektive - hier Landeskunde - neu zu entdecken. Aus diesem Grund bietet Kapitel 5 eine rgood practice`-Sammlung, die im Gegensatz zu `best practice` nicht beansprucht, die einzige beste Lösung gefunden zu haben. Unser Ziel war es, eine Auswahl an Lehrwerkinhalten aus der internationalen Praxis zu liefern. Die Sammlung bietet einen Einblick in eine Praxis über die eigenen Landesgrenzen hinweg und somit Möglichkeiten für eine Übertragung unterschiedlicher Aufgabenbeispiele und ihrer Didaktisierung für die eigene Unterrichtspraxis.

Es sind nämlich die Lehrkräfte, die das Angebot an Inhalten auf die Bedürfnisse der Lernenden vor Ort ausgestalten. In ihrem schulischen Kontext

27 Kommunikationsorientiert hier im Sinne von Entwicklung und Förderung der produktiven mündlichen Fertigkeit. 
- inklusive curricularen Rahmenbedingungen, Prüfungsvorgaben usw. - tragen sie und werden wohl auch in Zukunft die Verantwortung für die thematischinhaltliche Ausgestaltung des Unterrichts sowie die methodisch-didaktischen Schritte tragen, wie auch von Koreik betont wird:

In einem modernen, in den Sprachunterricht integrierten Landeskundeunterricht wird auch in Zukunft folgendermaßen unterrichtet werden müssen: adressatenspezifisch und in Abhängigkeit von Lehrzielen und den jeweiligen Curricula sowie den damit verbundenen Prüfungen und Tests [...]. Immer entsteht eine Gratwanderung zwischen informierendem, kommunikativem und zur Lernerautonomie hinführendem, auf Interaktion setzendem und auf interkulturelle Lernziele hinarbeitenden Unterricht, dessen Schwerpunktsetzung (im Rahmen von institutionellen Vorgaben) maßgeblich von der jeweiligen Lehrkraft vorgenommen werden muss. (Koreik 2013: 182)

Wie und inwiefern sich der curriculare Kontext auch auf Mikroebene - nämlich in der tatsächlichen schulischen Unterrichtspraxis - auswirkt, stellt bis heute ein Forschungsdesiderat dar. Die Zahl der Projekte, die erforschen, was mit Lehrmaterialien in der Unterrichtspraxis, also im Klassenraum passiert, wie die Studien von Röttger (2004), Maijala (2018) und Marcos Miguel (2015), ist bis heute als äußerst gering zu bezeichnen. Dabei wäre gerade ein solches Herangehen äußerst interessant, nicht nur um beschreiben zu können, wie groß der Einfluss von Kontext auf die Lehrwerkrezeption ist, sondern um überhaupt eine annähernde Vorstellung davon zu bekommen, welche Entscheidungen Lehrkräfte in ihrem schulischen Kontext, d.h. in ihrem Klassenzimmer, mit einer bestimmten Klasse, mit auf Schulebene inhaltlich und zeitlich festgelegten Prüfungen und mit ihren persönlichen Kompetenzen (nicht nur) im Bereich Landeskunde, treffen. 


\section{Literaturverzeichnis}

\section{Lehrwerke}

Altmayer, Claus (Hg.) (2016): Mitreden. Diskursive Landeskunde für Deutsch als Fremdund Zweitsprache. Von: Hamann, Eva / Magosch, Christine / Mempel, Caterina / Vondran, Björn / Zabel, Rebecca. Stuttgart: Klett.

Baas, Gert / Eunen, Kees van / Harshagen, Linda / Heidrich, Karoline / Hollander, Marcel den / Kreel, Willemijn van / Krijgsman, Arjan / Maser, Caroline / Mitzschke, Matthias / Schyns, Yolande / Vinzelberg, Anke / Vlak, Nienke (2013): TrabiTour 1 havo/vwo A t/m C. Groningen, Houten: Noordhoff.

Baas, Gert / Eunen, Kees van / Harshagen, Linda / Heidrich, Karoline / Hollander, Marcel den / Kreel, Willemijn van / Krijgsman, Arjan / Maser, Caroline / Mitzschke, Matthias / Schyns, Yolande / Vinzelberg, Anke / Vlak, Nienke (2014): TrabiTour 1 havo/vwo D t/m F. Groningen, Houten: Noordhoff.

Bachmann, Saskia / Gerhold, Sebastian / Müller, Bernd-Dietrich (1995): Sichtwechsel neu 1 - Mittelstufe Deutsch als Fremdsprache. Stuttgart: Klett.

Bayerlein, Oliver (2013): Landeskunde aktiv: Praktische Orientierungen für Deutschland, Österreich und die Schweiz. München: Verlag für Deutsch.

Behal-Thomsen, Heinke / Lundquist-Mog, Angelika / Mog, Paul (1993): Typisch deutsch? Arbeitsbuch zu Aspekten deutscher Mentalität. München: Langenscheidt.

Blok, Helen / Dekker, Marijke / Divendal, Christa / Ettema, Carine / Hassemer, Mireille / Hollander, Marcel den / Hollander, Tim den / Keeris, Manuala / Kreel, Willemijn van / Meuwissen, Renier / Schyns, Yolande / Veen, Zdenka ten (2017): Zugspitze. Textarbeitsbuch 4 vwo. Amersfoort: ThiemeMeulenhoff.

Borawitz, Monika / IJssel-Groffen, Clarije van / Meerman, Karin / Stek-de Bruin, Alie / Verhoeven, Stef / Verkerk-Schneider, Jessica (2013): Neue Kontakte 1-2 t/havo/vwo. Textbuch \& Arbeitsbuch A+B (havo/vwo). Groningen: Noordhoff.

Esterl, Ursula / Körner, Elke / Einhorn, Agnes/ Kubicka, Aleksandra / Jenkins-Krumm, Eva-Maria (2016): Team Deutsch. Deutsch für Jugendliche. Kursbuch 3. Stuttgart: Klett.

Esterl, Ursula / Körner, Elke / Einhorn, Agnes / Kubicka, Aleksandra / Jenkins-Krumm, Eva-Maria (2015): Team Deutsch. Deutsch für Jugendliche. Kursbuch 2. Stuttgart: Klett.

Fleer, Sarah / Koenig, Michael / Koithan, Ute / Sieber, Tanja (2018): Klasse! Deutsch für Jugendliche. Kursbuch A1. Stuttgart: Klett. 
Fleer, Sarah / Koithan, Ute / Schwieger, Bettina / Sieber, Tanja (2019): Klasse! Deutsch für Jugendliche. Kursbuch A2. Stuttgart: Klett.

Georgiakaki, Manuela / Bovermann, Monika / Graf-Riemann, Elisabeth / Seuthe, Christiane (2013): Beste Freunde. Deutsch für Jugendliche. Kursbuch A 1.1. München: Hueber.

Georgiakaki, Manuela / Graf-Riemann, Elisabeth / Schürmann, Anja / Seuthe, Christiane (2015): Beste Freunde. Deutsch für Jugendliche. Kursbuch A 2.2. München: Hueber.

Georgiakaki, Manuela / Graf-Riemann, Elisabeth / Seuthe, Christiane (2014): Beste Freunde. Deutsch für Jugendliche. Kursbuch A 1.2. München: Hueber.

Hahnfeld, Andrea / Heyse, Jochem / van IJssel-Groffen, Clarije / Meerman, Karin / Verkerk-Schneider, Jessica / Wegdam, Rob (2014): Neue Kontakte 3. havo/vwo. Textbuch \& Arbeitsbuch A + B. Groningen: Noordhoff.

Hansen, Margarete / Zuber, Barbara (1996): Zwischen den Kulturen. Strategien und Aktivitäten für landeskundliches Lehren und Lernen. Berlin/München: Langenscheidt.

Hunfeld, Hans / Piepho, Hans-Eberhard (1996): Elemente 1 - Das Lehrwerk für Deutsch als Fremdsprache. Köln: Dürr+Kessler.

Immers, Suzanne (2017): Unterrichtsmaterial Stadt- und Schulerkundungen. Nijmegen/ Duisburg-Essen: Nachbarsprache \& buurcultuur. Online: https://www.ru.nl/nachbar sprache/schulen/unterrichtsmaterial/ [Zugriff am 10.01.2019].

Jenkins, Eva-Maria / Clalüna, Monika / Fischer, Roland / Hirschfeld, Ursula (2006): Dimensionen. Magazin 3. Lernstationen 11-18. Ismaning: Hueber.

Jenkins, Eva-Maria / Fischer, Roland / Hirschfeld, Ursula / Hirtenlehner, Maria / Clalüna, Monika (2003a): Dimensionen. Magazin 2. Lernstationen 6-10. Ismaning: Hueber.

Jenkins, Eva-Maria / Fischer, Roland / Hirschfeld, Ursula / Hirtenlehner, Maria / Clalüna, Monika (2003b): Dimensionen. Lernstationen 6-10. Ismaning: Hueber.

Jentges, Sabine / Holtland, Laura (2018): DACH-Erkundungen: Die deutschsprachigen Länder kennenlernen. Nijmegen: Radboud Universiteit. Online: https://www.ru.nl/ nachbarsprache/schulen/unterrichtsmaterial/ [Zugriff am 20.09.2019].

Jin, Friederike / Rohrmann, Lutz (2014): prima plus+. Deutsch für Jugendliche A 1.2. Berlin: Cornelsen.

Jin, Friederike / Rohrmann, Lutz (2015): prima plus+. Deutsch für Jugendliche A 2.2. Berlin: Cornelsen.

Jin, Friederike / Rohrmann, Lutz (2016): prima plus+. Deutsch für Jugendliche A 1.1. Berlin: Cornelsen.

Kosacka, Małgorzata (2017): Meine Deutschtour. Zeszyt ćwiczeń do języka niemieckiego dla klasy siódmej szkoły podstawowej. Warszawa: Nowa Era.

Kosacka, Małgorzata (2018): Meine Deutschtour. Zeszyt ćwiczeń do języka niemieckiego dla klasy ósmej szkoły podstawowej. Warszawa: Nowa Era.

Kościelniak-Walewska, Ewa (2017): Meine Deutschtour. Podręcznik do języka niemieckiego dla klasy siódmej szkoły podstawowej. Warszawa: Nowa Era.

Kościelniak-Walewska, Ewa (2018): Meine Deutschtour. Podręcznik do języka niemieckiego dla klasy ósmej szkoły podstawowej. Warszawa: Nowa Era.

Kręciejewska, Elżbieta / Lisowska, Danuta / Serzysko, Cezary (2017): Mit links! 1. Podręcznik wieloletni dla klasy siódmej. Warszawa: Pearson Central Europe/WSiP.

Kręciejewska, Elżbieta / Lisowska, Danuta / Serzysko, Cezary (2017): Mit links! 1. Zeszyt ćwiczeń. Warszawa: Pearson Central Europe/WSiP. 
Lehrner-te Lindert, Elisabeth / Harshagen, Linda / Hoegen, Anne-Christine (2017): Na klar! Havo/vwo 1-2. Deel A \& B. s-Hertogenbosch: Malmberg.

Lehrner-te Lindert, Elisabeth / Gelissen, Gabrie / Schwill, Anna / Trommelen, Danique (2018): Na klar! Havo/vwo 3. Deel A \& B. s-Hertogenbosch: Malmberg.

Lundquist-Mog, Angelika (1996): Spielarten. Arbeitsbuch zur deutschen Landeskunde. Berlin/München: Langenscheidt.

Maccarini, Jocelyne / Boullot, Florian / Déchalotte, Aurélie / Hass, Nolwenn / Lowin Kropf, Bente (2018): Fantastisch! Deutsch für Jugendliche. Kursbuch A1. Stuttgart: Klett.

Matecki, Uta (2017): Dreimal Deutsch. In Deutschland - In Österreich - In der Schweiz. Lesebuch. Stuttgart: Klett.

Motta, Giorgio (2016): Deutsch echt einfach für Jugendliche. Kursbuch A1. Stuttgart: Klett. Pilaski, Anne / Fröhlich, Brigitta / Behal-Thomsen, Heinke (2011): Entdeckungsreise D-A$\mathrm{CH}$. Kursbuch zur Landeskunde. Berlin: Langenscheidt.

Pilaski, Anna / Fröhlich, Brigitta / Bolte-Costabiei, Christiane / Behal-Thomsen, Heinke (2013): Entdeckungsreise D-A-CH. Kursbuch zur Landeskunde. Stuttgart: Klett.

Reymont, Elżbieta / Sibiga, Agnieszka / Jezierska-Wiejak, Małgorzata (2017): Kompass Team 1. Podręcznik do języka niemieckiego dla klas VII-VIII. Warszawa: PWN.

Reymont, Elżbieta / Sibiga, Agnieszka / Jezierska-Wiejak, Małgorzata (2017): Kompass Team 1. Materiały ćwiczeniowe do języka niemieckiego dla klas VII-VIII. Warszawa: PWN.

Reymont, Elżbieta / Sibiga, Agnieszka / Jezierska-Wiejak, Małgorzata (2017): Kompass Team 2. Podręcznik do języka niemieckiego dla klas VII-VIII. Warszawa: PWN.

Reymont, Elżbieta / Sibiga, Agnieszka / Jezierska-Wiejak, Małgorzata (2017): Kompass Team 2. Materiały ćwiczeniowe do języka niemieckiego dla klas VII-VIII. Warszawa: PWN.

Seiffert, Christian (2009): Treffpunkt D-A-CH. Landeskundeheft (1-3). Berlin/München: Langenscheidt.

Serzysko, Cezary Michał / Kręciejewska, Elżbieta / Sekulski, Birgit (2018): Mit links! 2. Podręcznik wieloletni dla klasy siódmej. Warszawa: Pearson Central Europe/WSiP. Serzysko, Cezary Michał / Kręciejewska, Elżbieta / Sekulski, Birgit (2018): Mit links! 2. Zeszyt ćwiczeń. Warszawa: Pearson Central Europe/WSiP.

Specht, Franz / Heuer, Wiebke / Pasewalck, Silke / Neidlinger, Dieter / Dahmen, Kristine (2012): Zwischendurch mal Landeskunde. Kopiervorlagen. Ismaning: Hueber.

\section{Sekundärliteratur}

ABCD-Thesen zur Rolle der Landeskunde im Deutschunterricht (1990). In: IDV-Rundbrief 45 (1990), 15-18.

Abel, Brigitte (1988): Wie kulturspezifisch sind regionale Lehrwerke? In: Jahrbuch Deutsch als Fremdsprache 14, 238-258.

Adamczak-Krysztofowicz, Sylwia / Jentges, Sabine / Stork, Antje (2015): Fremde und eigene Gewässer: Interkulturelles Lernen im Fremdsprachenunterricht. In: Cerri, Chiara / Jentges, Sabine (Hg.): Raumwahrnehmung, interkulturelles Lernen und Fremdsprachenunterricht. Baltmannsweiler: Schneider Hohengehren, 11-29. 
Altmayer, Claus (2002): Kulturelle Deutungsmuster in Texten. Prinzipien und Verfahren einer kulturwissenschaftlichen Textanalyse im Fach Deutsch als Fremdsprache. In: Zeitschrift für Interkulturellen Fremdsprachenunterricht 6 (3). Online: http://tujour nals.ulb.tu-darmstadt.de/index.php/zif/article/view/585 [Zugriff am 20.04.2019].

Altmayer, Claus (2004): Kultur als Hypertext. Zu Theorie und Praxis der Kulturwissenschaft im Fach Deutsch als Fremdsprache. München: Iudicium.

Altmayer, Claus (2006): Kulturelle Deutungsmuster als Lerngegenstand. Zur kulturwissenschaftlichen Transformation der `Landeskunde`. In: Fremdsprachen lehren und lernen 35, 44-59.

Altmayer, Claus (2007): Von der Landeskunde zur Kulturwissenschaft - Innovation oder Modetrend? In: Germanistische Mitteilungen. Zeitschrift des Belgischen Germanistenund Deutschlehrerverbandes 65, 7-21.

Altmayer, Claus (2008): Von der sinterkulturellen Kompetenz« zum `kulturbezogenen Deutungslernen`. Plädoyer für eine kritische Transformation des interkulturellen Ansatzes` in der Landeskunde. In: Schulz, Renate / Tschirner, Erwin: Interkulturelle Kompetenz. München: Iudicium, 28-41.

Altmayer, Claus (2013): Die DACH-Landeskunde im Spiegel aktueller kulturwissenschaftlicher Ansätze. In: Demmig, Silvia / Hägi, Sara / Schweiger, Hannes (Hg.): DACHLandeskunde. Theorie - Geschichte - Praxis. München: Iudicium, 15-31.

Altmayer, Claus / Hamann, Eva / Magosch, Christine / Mempel, Caterina / Vondran, Björn / Zabel, Rebecca (o. J.): Einführung »Mitreden«. Online: https://www.klett-sprachen.de/ download/9744/675267_Einleitung.pdf [Zugriff am 10.01.2019].

Androutsopoulos, Jannis (2008): Linguistic landscapes: Visuelle Mehrsprachigkeitsforschung als Impuls an die Sprachpolitik. Goethe Institut: Städte - Sprachen - Kulturen. Online: https://jannisandroutsopoulos.files.wordpress.com/2011/05/j-a-2008-linguis tic-landscapes.pdf [Zugriff am 10.01.2019].

Bachmann-Medick, Doris (2006): Cultural Turns. Neuorientierungen in den Kulturwissenschaften. Reinbek: Rowohlt.

Badstübner-Kizik, Camilla (2007): Kontaktdidaktik - ein mögliches Konzept für den DaFUnterricht in Mitteleuropa? In: Schmitz, Walter / Joachimsthaler, Jürgen (Hg.): Zwischeneuropa / Mitteleuropa. Sprache und Literatur in interkultureller Konstellation, Dresden: Thelem, 708-716.

Badstübner-Kizik, Camilla (2010): Kontaktdidaktik und ihre empirischen Implikationen. In: Zeitschrift für Interkulturellen Fremdsprachenunterricht 15/2010/2, 99-112. Online: http://tujournals.ulb.tu-darmstadt.de/index.php/zif/article/view/158/153 [Zugriff am 10.01.2019].

Badstübner-Kizik, Camilla / Hille, Almut (Hg.) (2015): Kulturelles Gedächtnis und Erinnerungsorte im hochschuldidaktischen Kontext. Perspektiven für das Fach Deutsch als Fremdsprache, Frankfurt a.M.: Peter Lang.

Badstübner-Kizik, Camilla / Janíková, Vera (Hg.) (2018): Linguistic landscape und Fremdsprachendidaktik. Posener Beiträge zur Angewandten Linguistik 10. Berlin: Peter Lang.

Barkowski, Hans / Fritsche, Michael / Göbel, Richard (1980): Deutsch für ausländische Arbeiter: Gutachten zu ausgewählten Lehrwerken. Mainz: Werkmeister.

Bausch, Karl-Richard / Christ, Herbert / Krumm, Hans-Jürgen (Hg.) (1994): Interkulturelles Lernen im Fremdsprachenunterricht. Arbeitspapiere der 14. Frühjahrskonferenz zur Erforschung des Fremdsprachenunterrichts. Tübingen: Narr. 
Bausch, Karl-Richard / Krumm, Hans-Jürgen / Burwitz-Melzer, Eva / Mehlhorn, Grit (Hg.) (2016): Handbuch Fremdsprachenunterricht. Tübingen: Francke.

Beier, Rudolf / Möhn, Dietmar (1981): Vorüberlegungen zu einem `Hamburger Gutachten . In: Fachsprache 3/4, 112-50.

Berger, Maria Cr. / Martini, Maddalena (2005): Generation E. Deutschsprachige Landeskunde im europäischen Kontext. Stuttgart: Klett.

Biechele, Markus / Meinert, Roland (2001): Handbuch zur Spracharbeit (Goethe-Institut). Teil 5: Erlebte Landeskunde. Ismaning: Hueber.

Bleyhl, Werner (1994): Das Lernen von Fremdsprachen ist interkulturelles Lernen. In: Bausch, Karl-Richard / Christ, Herbert / Krumm, Hans-Jürgen (Hg.): Interkulturelles Lernen im Fremdsprachenunterricht. Arbeitspapiere der 14. Frühjahrskonferenz zur Erforschung des Fremdsprachenunterrichts. Tübingen: Narr, 9-20.

Bolten, Jürgen (2007): Was heißt interkulturelle Kompetenz? Perspektiven für die internationale Personalentwicklung. In: Kuenzer, Vera / Berninghausen, Jutta (Hg.): Wirtschaft als interkulturelle Herausforderung. Frankfurt: Verlag für interkulturelle Kommunikation, 21-42.

Boonen, Ute K. / Jentges, Sabine / Sars, Paul (2018): Grenzenlos Lernen in der Euregio Rhein-Waal - Das deutsch-niederländische Schulaustauschprojekt Nachbarsprache \& buurcultuur. In: nachbarsprache niederländisch, 97-107.

Börner, Otfried / Edelhoff, Christoph / Rebel, Karlheinz / Schmidt, Torben / Schröder, Konrad (2011): Funktion und Profil von Lehrwerken in der Epoche von Standards und Kompetenzen. In: FLuL - Fremdsprachen Lehren und Lernen 40 (2), 31-48.

Brade, Janine / Krull, Danny (Hg.) (2016): 45 Lern-Orte in Theorie und Praxis. Außerschulisches Lernen in der Grundschule für alle Fächer und Klassen. Baltmannsweiler: Schneider Hohengehren.

Burwitz-Melzer, Eva / Königs, Frank G. / Riemer, Claudia (2015): Lernen an allen Orten?: Die Rolle der Lernorte beim Lehren und Lernen von Fremdsprachen. Arbeitspapiere der 35. Frühjahrskonferenz zur Erforschung des Fremdsprachenunterrichts. Tübingen: Narr.

Butzkamm, Wolfgang (1993): Psycholinguistik des Fremdsprachenunterrichts: Von der Muttersprache zur Fremdsprache. Tübingen, Basel: Francke.

Butzkamm, Wolfgang (2005): Der Lehrer ist unsere Chance: Wie Schüler ihren Fremdsprachenunterricht erleben. Essen: Geissler.

Butzkamm, Wolfgang / Caldwell, John A. W. (2009): The Bilingual Reform: A Paradigm Shift in Foreign Language Teaching. Tübingen: Gunter Narr Verlag.

Cenoz, Jasone / Gorter, Durk (2008): The linguistic landscape as an additional source of input in second language acquisition. In: IRAL 46/2008/3, 267-287.

Cerri, Chiara / Dausend, Henriette (2015): `Raum` - mehrperspektivischer Zugang zu einem vermeintlich eindimensionalen Begriff. In: Cerri, Chiara / Jentges, Sabine (Hg.): Raumwahrnehmung, interkulturelles Lernen und Fremdsprachenunterricht. Baltmannsweiler: Schneider Hohengehren, 33-51.

Cerri, Chiara / Delhey, Yvonne / Jentges, Sabine (2015): Raumdenken: Interkulturelles Lernen im europäischen Kontext. In: Cerri, Chiara / Jentges, Sabine (Hg.): Raumwahrnehmung, interkulturelles Lernen und Fremdsprachenunterricht. Baltmannsweiler: Schneider Hohengehren, 3-10. 
Cerri, Chiara / Jentges, Sabine (2015a): (Urbane) Räume als Schlüssel zum kulturgeprägten Lernen. In: Acharya, Swati u.a. (Hg.), IDT 2013, Band 3.1: Kultur, Literatur, Landeskunde, Bolzano, 147-162.

Cerri, Chiara / Jentges, Sabine (2015b): Erlebter Raum, erlebte Kultur. Räume als Schlüssel zum landeskundlichen Lernen im Fremdsprachenunterricht. In: Cerri, Chiara / Jentges, Sabine (Hg.): Raumwahrnehmung, interkulturelles Lernen und Fremdsprachenunterricht. Baltmannsweiler: Schneider Hohengehren, 97-120.

Ciepielewska-Kaczmarek, Luiza (2016): Möglichkeiten und Grenzen interkulturellen Lernens im institutionalisierten DaF-Unterricht. Poznań: Wydział Neofilologii UAM w Poznaniu. DOI: 10.14746/9788394601799.

Clalüna, Monika / Fischer, Roland / Hirschfeld, Ursula (2007): Alles unter einem D-A-CHL? Oder: Wie viel Plurizentrik verträgt ein Lehrwerk? In: Fremdsprache Deutsch 37, 38-45.

Clalüna, Monika / Langner, Michael (1998): Landeskunde - deutschsprachige Länder: Schweiz. Regensburg: Dürr+Kessler.

CVE (2012): Syllabus moderne vreemde talen vwo centraal examen 2014. Utrecht: College voor Examens. Online: : https://www.examenblad.nl/examenstof/syllabus-2015-moder ne-vreemde-3/2015/vwo/f=/mvt_def_versie_vwo_2015.pdf [Zugriff am 25.11.2018].

Deardoff, Darla K. (2006): Identification and Assessment of Intercultural Competence as a Student Outcome of Internationalization. In: Journal of Studies of International Education 10/2006/3. Online: https://journals.sagepub.com/doi/abs/10.1177/10283153062 87002 [Zugriff am 10.01.2019].

Delhey, Yvonne / Jentges, Sabine (2013): „Von Wahrnehmung zu Wissen: Interkulturelles Lernen im urbanen Raum« Ein Lehrprojekt für den Einsatz im Fremdsprachenunterricht. In: Hille, Almut / Langer, Benjamin (Hg.): Erzählte Städte. Beiträge zur Forschung und Lehre in der europäischen Germanistik. München: Iudicium, 179-194.

Demmig, Silvia / Hägi, Sara / Schweiger, Hannes (Hg.) (2013): DACH-Landeskunde: Theorie - Geschichte - Praxis. München: Iudicium.

DIA (2010): Rapport belevingsonderzoek Duits. Amsterdam: Duitslandinstituut Amsterdam. Online: http://www.duitslandinstituut.nl/art/uploads/files/2011/Rapport\%20 belevingsonderzoek.pdf [Zugriff am 01.09.2017].

Dickel, Mirko / Glasze, Georg (2009): Rethinking Excursions - Konzepte und Praktiken einer konstruktivistisch orientierten Exkursionsdidaktik. In: Dickel, Mirko / Glasze, Georg (Hg.): Vielperspektivität und Teilnehmererziehung - Richtungsweiser einer Exkursionsdidaktik. Wien: LIT, 3-14.

Dresing, Pirkko Friederike / Bechaus, Carina / Möllenkamp, Mareike / Ballweg, Sandra u. a. (2017): Wie nehmen neu zugewanderte Menschen in Deutschland ihre sprachliche Umgebung wahr? Zum Umgang von Deutschlernenden mit Text-Bild-Kombinationen im Alltag. In: Materialien Deutsch als Fremd- und Zweitsprache 98, 117-148.

Engel, Ulrich / Halm, Wolfgang / Krumm, Hans-Jürgen / Ortmann, Wolf-Dieter / Picht, Robert / Rall, Dietrich / Schmidt, Walter / Stickel, Gerhard / Vorderwülbecke, Klaus / Wierlacher, Alois (1977): Mannheimer Gutachten zu ausgewählten Lehrwerken Deutsch als Fremdsprache. Band 1. Heidelberg: Julius Groos.

Engel, Ulrich / Krumm, Hans-Jürgen / Wierlacher, Alois (1979): Mannheimer Gutachten zu ausgewählten Lehrwerken Deutsch als Fremdsprache. Band 2. Heidelberg: Julius Groos. 
Faigle, Iris (2006): Rundum. Einblicke in die deutschsprachige Kultur. Stuttgart: Klett. Faigle, Iris (2007): Willkommen bei uns. Landeskundliche Aspekte der deutschsprachigen Länder. Recanati: Eli.

Fery, Renate / Raddatz, Volker (Hg.) (2000): Lehrwerke und ihre Alternativen. Frankfurt a. M.: Peter Lang.

Fischer, Roland / Hackl, Wolfgang / Hirtenlehner, Maria / Rusch, Paul (1998): Landeskunde - deutschsprachige Länder: Österreich. Regensburg: Dürr+Kessler.

Fornoff, Roger (2016): Landeskunde und kulturwissenschaftliche Gedächtnisforschung. Erinnerungsorte des Nationalsozialismus im Unterricht Deutsch als Fremdsprache. Baltmannsweiler: Schneider Hohengehren.

Fornoff, Roger / Koreik, Uwe: Ist der kulturwissenschaftliche und kulturdidaktische Bezug auf die Nation überholt? DACH-Landeskunde, Globalisierung und Erinnerungsorte. Eine Intervention. In: Materialien Deutsch als Fremdsprache (im Druck).

Fornoff, Roger / Koreik, Uwe: Landeskunde/Kulturstudien und kulturelles Lernen im Fach DaF/DaZ - Eine Bestandsaufnahme und kritische Positionierung. In: ZIF 2020 (Jubiläumsausgabe) (in Vorbereitung).

Freudenstein, Reinhold (2001): Fremdsprachen lernen ohne Lehrbuch. Zur Geschichte, Gegenwart und Zukunft fremdsprachlicher Unterrichtsmaterialien. In: Praxis 48, 8-19.

Funk, Hermann (2004): Qualitätsmerkmale von Lehrwerken prüfen - ein Verfahrensvorschlag. In: Babylonia 3/04, 41-47. Online: www.babylonia-ti.ch [Zugriff am 15.03. 2019].

Funk, Hermann (2010): Lehrwerkforschung. In: Hallet, Wolfgang / Königs, Frank G. (Hg.): Handbuch Fremdsprachendidaktik. Seelze-Velber: Klett Kallmeyer, 364-368.

Funk, Hermann (2016): Lehrwerke. In: Bausch, Karl-Richard / Krumm, Hans-Jürgen / Burwitz-Melzer, Eva / Mehlhorn, Grit (Hg.): Handbuch Fremdsprachenunterricht. Tübingen: Francke, 435-441.

Garton, Sue / Graves, Kathleen (2014): International Perspectives on Materials in ELT. London: Palgrave Macmillan.

Gehring, Wolfgang / Stinshoff, Elisabeth (2010): Außerschulische Lernorte des Fremdsprachenunterrichts. Braunschweig: Diesterweg.

Gemeinsamer europäischer Referenzrahmen für Sprachen: Lehren, lernen, beurteilen. Online: https://www.goethe.de/Z/50/commeuro/i1.htm [Zugriff am 10.01.2019].

Gießing, Jürgen (2003): Fremdsprachen lernen trotz Lehrbuch. Weitere Anmerkungen zur Rolle des Lehrwerks beim Fremdsprachenlernen. In: Praxis des neusprachlichen Unterrichts 1/2003, 91-93.

Gießing, Jürgen (2004): Zankapfel Lehrbuch. Für und wider ein etabliertes Unterrichtsmittel. In: Praxis Fremdsprachenunterricht 2/2004, 82-84.

Gorter, Durk (Hg.) (2006): Linguistic Landscape: A New Approach to Multilingualism. Clevedon: Channel.

Grünewald, Andreas (2017): Community Language Learning. In: Surkamp, Carola (Hg.): Metzler Lexikon Fremdsprachendidaktik. Ansätze - Methoden - Grundbegriffe. Stuttgart, Weimar: J.B. Metzler, 35-36.

Guerrettaz, Anne Marie / Johnston, Bill (2013): Materials in the classroom ecology. In: The Modern Language Journal 97/3, 779-796.

Gürttler, Karin / Steinfeld, Thomas (1990): Landeskunde - ein unmögliches Fach aus Deutschland. In: Informationen Deutsch als Fremdsprache 3, 250-258. 
Hackl, Wolfgang / Langner, Michael / Simon-Pelanda, Hans (1997): Integrierende Landeskunde - ein (gar nicht so neuer) Begriff. In: Theorie und Praxis. Österreichische Beiträge zu Deutsch als Fremdsprache 1, 17-34.

Harwood, Nigel (2013): Content, consumption, and production: Three levels of textbook research. In: Harwood, Nigel (Hg.): English Language Teaching Textbooks: Content, Consumption, Production. Basingstoke, UK: Palgrave Macmillan, 1-41.

Helbig, Gerhard (Hg.): Deutsch als Fremdsprache. Ein internationales Handbuch. Berlin, New York: Walter de Gruyter.

Herrmann-Teubel, Yvonne / Jarząbek, Alina Dorota / Shafer, Naomi (Hg.)(2017): IDVMagazin: Das DACH-Prinzip 92/2017.

Hoch, Barbara (2016): Kulturreflexives Lernen im Deutsch-als-Fremdsprache-Unterricht: Eine Analyse deutscher, französischer und niederländischer DaF-Lehrwerke für Jugendliche. Mannheim: Universität Mannheim. Online: https://ub-madoc.bib.unimannheim.de/40683/ [Zugriff am 10.01.2019].

Hoch, Barbara / Jentges, Sabine / Tammenga-Helmantel, Marjon (2016): Beantworte die Fragen auf Niederländisch. Zielsprachengebrauch in niederländischen DaF-Lehrwerken. In: Info DaF, Jahrgang 43. Themenschwerpunkt: Lehrwerke beim Deutsch lernen weltweit. München: Iudidium, 599-622.

Huisman, Sandra (2017): Germanist voor de klas. De belangstelling voor de universitaire lerarenopleiding Duits in Nederland. In: Jentges, Sabine / Sars, Paul / Wielenga, Friso / Wilp, Markus (Hg.): Grenzüberschreitend forschen. Zusammenfassungen ausgewählter Abschlussarbeiten des Masterstudiengangs Niederlande-Deutschland-Studien. Münster: WWU, 141-160.

IDV (2013): Dach-Prinzip. Was ist das DACH-Prinzip? Online: https://idvnetz.org/dachlonline/dachl-im-fach-dafdaz/dach-prinzip [Zugriff am 7.1.2019].

Jarząbek, Alina Dorota (2017): DACHL - Eindrücke, Erfahrungen, Erinnerungen. In: IDVMagazin 92, 14-19.

Jentges, Sabine (2018): Kennis van land en cultuur - een introductie. In: Nuffic Academie: LinQ- Landeskunde/Civilisation. Online: https://www.internationalisering.nl/wp-con tent/uploads/2018/08/Theoretisch-kader-module-KvTLC.pdf [Zugriff am 7.1.2019].

Jentges, Sabine / Sars, Paul (2017): Deutsch als Fremdsprache in den Niederlanden und in den deutsch-niederländischen Grenzregionen: Position, Herausforderungen und Perspektiven. In: Muttersprache 1/2017, 43-54.

Jentges, Sabine / Sars, Paul (2018): Ich sehe was, was du nicht siehst ... Urbanes Raumerleben und linguistic landscaping in und für deutsch-niederländische/n Schulaustauschprojekte/n. In: Badstübner-Kizik, Camilla / Janíková, Vera (Hg.): Linguistic landscape und Fremdsprachendidaktik. Posener Beiträge zur Angewandten Linguistik 10. Berlin: Peter Lang, 21-54.

Kast, Bernd / Neuner, Gerhard (1994) (Hg.): Zur Analyse, Begutachtung und Entwicklung von Lehrwerken für den fremdsprachlichen Deutschunterricht. Berlin u.a.: Langenscheidt.

Kistermann, Rainer (2008): City portrait - Schüler erstellen einen kurzen Informationsfilm. In: Praxis Englisch 6, 32-25.

Koch, Leo (1999): Landeskunde - deutschsprachige Länder: Begleitband. Anregungen Arbeitsformen - Merkblätter. Regensburg: Dürr+Kessler. 
Koenig, Michael (2010): Lehrwerkarbeit. In: Hallet, Wolfgang / Königs, Frank G. (Hg.): Handbuch Fremdsprachendidaktik. Seelze-Velber: Klett / Kallmeyer, 177-182.

Koreik, Uwe (2013): Landeskunde. In: Ahrenholz, Bernt / Oomen-Welke, Ingelore: Handbuch Deutsch als Fremdsprache (Deutschunterricht in Theorie und Praxis, 10). Baltmannsweiler: Schneider Hohengehren, 178-186.

Koreik, Uwe (2018): Das deutsche `Wirtschaftswunder`. Mythos, Legende oder ein Erinnerungsort - die Relevanz für Deutsch als Fremd- und Zweitsprache? In: Schiedermair, Simone (Hg.): Deutsch als Fremd- und Zweitsprache \& Kulturwissenschaft. Zugänge zu sozialen Wirklichkeiten. München: Iudicium, 27-46.

Koreik, Uwe / Pietzuch, Jan Paul (2010): Entwicklungslinien landeskundlicher Ansätze und Vermittlungskonzepte. In: Krumm, Hans-Jürgen / Fandrych, Christian / Hufeisen, Britta / Riemer, Claudia (Hg.): Deutsch als Fremd- und Zweitsprache. Ein internationales Handbuch. Band 2. Berlin et al.: De Gruyter, 1441-1454.

Krumm, Hans-Jürgen (1985): Stockholmer Kriterienkatalog zur Beurteilung von Lehrwerken des Faches Deutsch als Fremdsprache in den nordischen Ländern. Stockholm: Universität Uppsala / Goethe-Institut.

Krumm, Hans-Jürgen (1998): Landeskunde Deutschland. DACH oder Europa? In: Informationen Deutsch als Fremdsprache 5, 523-544.

Krumm, Hans-Jürgen (2010): Lehrwerke im Deutsch als Fremd- und Deutsch als Zweitsprache-Unterricht. In: Krumm, Hans-Jürgen / Fandrych, Christian / Hufeisen, Britta / Riemer, Claudia: Deutsch als Fremd- und Zweitsprache. Ein internationales Handbuch. Berlin / New York, 1215-1229.

Krumm, Hans-Jürgen (2017): Von ABCD zu DACHL. In: IDV-Magazin: Das DACHPrinzip 92/2017, 6-13.

Krumm, Hans-Jürgen / Fandrych, Christian / Hufeisen, Britta / Riemer, Claudia (Hg.) (2010): Deutsch als Fremd- und Zweitsprache. Ein internationales Handbuch. Berlin, New York: de Gruyter.

Krumm, Hans-Jürgen / Ohms-Duszenko, Maren (2001): Lehrwerkproduktion, Lehrwerkanalyse, Lehrwerkkritik. In: Helbig, Gerhard et al. (Hg.): Deutsch als Fremdsprache. Ein internationales Handbuch. 2. Halbband. Berlin, New York: de Gruyter, 1029-1041.

Kurtz, Jürgen (2011): Zur Einführung in den Themenschwerpunkt. In: Gnutzmann, Claus / Königs, Frank G. / Küster, Lutz (Hg.): Fremdsprachen Lehren und Lernen. Lehrwerkkritik, Lehrwerkverwendung, Lehrwerkentwicklung. Tübingen: Narr Verlag, 3-15.

Landry, Rodrigue / Bourhis, Richard Y. (1997): Linguistic landscape and ethnolinguistic vitality: An empirical study. In: Journal of Language and Social Psychology 16. Sage Publishing: Thousand Oaks, 23-49.

Luke, Carmen / de Castell, Suzanne / Luke, Allan. (1989): Beyond Criticism: The Authority of the School Textbook. In: de Castell, Suzanne / Luke, Allan / Luke, Carmen (Hg.): Language, authority and criticism: Readings on the school textbook. London: Falmer Press, 245-260.

Lütge, Christiane (2016): Lehr-/Lernmaterialien und Medien zum Aufbau interkultureller Kompetenzen. In: Bausch, Karl-Richard / Krumm, Hans-Jürgen / Burwitz-Melzer, Eva / Mehlhorn, Grit (Hg.): Handbuch Fremdsprachenunterricht. Tübingen: Francke, 456459. 
Maijala, Minna (2018): Die Rezeption eines überregionalen Lehrwerks im finnischen DaFUnterricht. InfoDaF. Online: https://doi.org/10.1515/infodaf-2018-0086 [Zugriff am 15.10.2019].

Maijala, Minna / Tammenga-Helmantel, Marjon (2016): Regionalität als Stärke? Eine Analyse von finnischen und niederländischen DaF-Lehrwerken. In: Info DaF 5/2016, 537-565.

Maijala, Minna / Tammenga-Helmantel, Marjon / Donker, Eva (2016): Das DACH-Konzept in finnischen und niederländischen DaF-Lehrwerken. In: Zielsprache Deutsch 43/1, 333.

Marcos Miguel, Nausica (2015): Textbook consumption in the classroom: Analyzing a classroom corpus. In: Procedia - Social and Behavioral Sciences 198, 309-319.

Marten, Heiko F. / Saagpakk, Maris (Hg.) (2017): Linguistic landscapes und Spot German an der Schnittstelle von Sprachwissenschaft und Deutschdidaktik. München: Iudicium.

McIver, Nick (2009): Bookreview: Meddings, Luke / Thornbury, Scott: Teaching Unplugged: Dogme in English Language Teaching: ELT Journal, 4, 419-421. Online: https://doi.org/10.1093/elt/ccp072 [Zugriff am 10.02.2019].

Meddings, Luke / Thornbury, Scott (2009): Teaching Unplugged. Dogme in English Language Teaching. Peaslake: Delta Publishing.

Mitzschke, Volker (1998): Landeskunde - deutschsprachige Länder: Deutschland. Regensburg: Dürr+Kessler.

Mog, Paul (1992): Die Deutschen in ihrer Welt: Tübinger Modell einer integrativen Landeskunde. München: Langenscheidt.

Neuner, Gerhard (1994): Lehrwerkforschung, Lehrwerkkritik. In: Kast, Bernd / Neuner, Gerhard (Hg.): Zur Analyse, Begutachtung und Entwicklung von Lehrwerken für den fremdsprachlichen Deutschunterricht. Berlin u. a.: Langenscheidt, 8-22.

Neuner, Gerhard (2003): Lehrwerke. In: Bausch, Karl-Richard / Christ, Herbert / Krumm, Hans-Jürgen (Hg.): Handbuch Fremdsprachenunterricht. Tübingen, Basel: Francke, 399-402.

Nieweler, Andreas (2010): Lehrwerk. In: Surkamp, Carola (Hg.): Metzler Lexikon Fremdsprachendidaktik. Ansätze - Methoden - Grundbegriffe. Stuttgart: Metzler, 175-176.

Nieweler, Andreas (2017a): Lehrwerke. In: Surkamp, Carola (Hg.): Metzler Lexikon Fremdsprachendidaktik. Ansätze - Methoden - Grundbegriffe. Stuttgart: Metzler, 206-208.

Nieweler, Andreas (2017b): Lehrwerkanalyse. In: Surkamp, Carola (Hg.): Metzler Lexikon Fremdsprachendidaktik. Ansätze - Methoden - Grundbegriffe. Stuttgart: Metzler, 208-209.

Nolda, Sigrid (1995): Grenzen des interkulturellen Lernens - nicht nur im Anfangsunterricht. In: Burger, Günter (Hg.): Fremdsprachenunterricht in der Erwachsenenbildung: Perspektiven und Alternativen für den Anfangsunterricht. Ismaning: Hueber, 93-104.

Nuffic Academie (2017): LinQ- Landeskunde/Civilisation. Online: https://www.internatio nalisering.nl/modules/linq-landeskunde-civilisation/ [Zugriff am 7.1.2019].

Over, Ulf / Mienert, Malte / Grosch, Christiane / Hany, Ernst (2008): Interkulturelle Kompetenz: Begriffsklärung und Methoden der Messung. In: Ringeisen, Tobias / 
Buchwald, Petra / Schwarzer, Christine (Hg.): Interkulturelle Kompetenz in Schule und Weiterbildung. Berlin et al.: LIT, 65-79.

Pasewalck, Silke / Neidlinger, Dieter (2018): Kulturelles Lernen in (historisch) plurikulturellen Regionen. Methodologische Überlegungen zur Arbeit mit Erinnerungsorten und Linguistic Landscapes am Beispiel des Baltikums. In: Schiedermair, Simone (Hg.): Deutsch als Fremd- und Zweitsprache \& Kulturwissenschaft. Zugänge zu sozialen Wirklichkeiten. München: Iudicium, 47-70.

Pauldrach, Andreas (1992): Eine unendliche Geschichte. Anmerkungen zur Situation der Landeskunde in den 90er Jahren. In: Fremdsprache Deutsch 6, 4-15.

Pfeiffer, Waldemar (2012): Wie in Kafkas Schloss. In: Adamczak-Krysztofowicz, Sylwia / Stork, Antje (Hg.): Multikompetent - multimedial - multikulturell? Aktuelle Tendenzen in der DaF-Lehrerausbildung. Frankfurt a. M.: Peter Lang, 15-30.

Pfeiffer, Waldemar (2008): Die Qual der Wahl oder: Was muss ich tun, um ein geeignetes Lehrwerk zu wählen. In: Janoszczyk, Jolanta / Krzysiak, Lucyna / Żmudzki, Jerzy (Hg.): Deutsch lernen und lehren mit Lehrwerken. Vergangenheit. Gegenwart. Zukunft. Lublin: Wydawnictwo UMCS, 59-63.

Picht, Robert (1995): Kultur- und Landeswissenschaft. In: Bausch, Karl-Richard / Christ, Herbert (Hg.): Handbuch Fremdsprachenunterricht. Tübingen: Francke, 66-73.

Pucharski, Astrid (2009): DACH(L) neu: Perspektiven und Chancen eines Neustarts. In: Visionen. Gegenwart und Zukunft von $\mathrm{DaF} / \mathrm{DaZ}$ in Österreich. ÖDaF-Mitteilungen (Sonderheft zur IDT 2009), 110-117.

Purschke, Christopher (2017): Crowdsourcing the linguistic landscape of a multilingual country. Introducing Lingscape in Luxembourg. In: Hundt, Markus / Purschke, Christoph / Ziegler, Evelyn (Hg.): Language areas: configurations, interactions, perceptions. In: Linguistics Online 85/6/17, 181-202. Online: https://bop.unibe.ch/linguistik-online/ article/view/4086/6131 [Zugriff am 20.08.2017].

Pütz, Martin / Mundt, Neele (Hg.) (2018): Expanding the Linguistic Landscape. Linguistic Diversity, Multimodality and the Use of Space as a Semiotic Resource. Bristol, Blue Ridge Summit: Multilingual Matters. Online: https://doi.org/10.21832/978178892216 6-fm [Zugriff am 20.08.2017].

Rathje, Stefanie (2006): Interkulturelle Kompetenz - Zustand und Zukunft eines umstrittenen Konzepts. In: Zeitschrift für Interkulturellen Fremdsprachenunterricht 11/3. Online: https://tujournals.ulb.tu-darmstadt.de/index.php/zif/article/view/396/384 [Zugriff am 10.01.2019].

Richter, Regina (1998): Interkulturelles Lernen im DaF-Unterricht am Beispiel von "Sichtwechsel neu«, »Spielarten« und »Elemente«. In: Deutsch als Fremdsprache 35/1, 45-51.

Roche, Jörg / Röhling, Jürgen (Hg.) (2014): Erinnerungsorte und Erinnerungskulturen. Konzepte und Perspektiven für die Sprach- und Kulturvermittlung. Baltmannsweiler: Schneider Hohengehren.

Rösler, Dietmar (1994): Deutsch als Fremdsprache. Stuttgart: Metzler.

Rösler, Dietmar (2012): Deutsch als Fremdsprache. Eine Einführung. Stuttgart: Metzler. Rösler, Dietmar (2013): Lehrmaterial für Deutsch als Fremdsprache. In: Oomen-Welke, Ingelore / Ahrenholz, Bernt (Hg.): Deutschunterricht in Theorie und Praxis: Deutsch als Fremdsprache. Hohengehren: Schneider, 212-220. 
Rösler, Dietmar / Schart, Michael (2016): Die Perspektivenvielfalt der Lehrwerkanalyse und ihr weißer Fleck. In: Info DaF, 43/2016, 483-493.

Rösler, Dietmar / Würffel, Nicola (2013): Digitale Medien. In: Oomen-Welke, Ingelore / Ahrenholz, Bernt (Hg.): Deutschunterricht in Theorie und Praxis: Deutsch als Fremdsprache. Baltmannsweiler: Schneider Hohengehren, 252-260.

Rösler, Dietmar / Würffel, Nicola (2014): Lernmaterialien und Medien. München: Goethe Institut/Klett.

Rössler, Andrea (2010): Interkulturelle Kompetenz. In: Meißner, Franz-Josef / Tesch, Bernd (Hg.): Spanisch kompetenzorientiert unterrichten. Kallmeyer / Klett: Seelze, 137-149.

Röttger, Evelyn (2004): Interkuturelles Lernen im Fremdsprachenunterricht. Das Beispiel Deutsch als Fremdsprache in Griechenland. Hamburg: Kovac.

Sambanis, Michaela (2009): Stärken und Schwächen des Fremdsprachenunterrichts. Aus der Sicht von Sekundarstufenschüler/innen. In: Praxis Fremdsprachenunterricht 3, 912.

Sars, Paul / Boonen, Ute K. / Jentges, Sabine (2018): Samen elkaars taal en cultuur leren kennen: Het project »Nachbarsprache \& buurcultuur «. In: Levende Talen Magazine 03/ 105, 16-10. Online: http://www.lt-tijdschriften.nl/ojs/index.php/ltm/article/view/177 0/1377 [Zugriff am 15.03.2019].

Schäfer, Werner (2003): Unterrichten ohne Lehrbuch? Einige unzeitgemäße Bemerkungen. In: Praxis 50, 305-311.

Schiedermair, Simone (Hg.) (2018): Deutsch als Fremd- und Zweitsprache \& Kulturwissenschaft. Zugänge zu sozialen Wirklichkeiten. München: Iudicium.

Schmidt, Karin / Schmidt, Sabine (2007): Erinnerungsorte. Deutsche Geschichte im DaFUnterricht. Berlin: Cornelsen.

Schmidt, Siegfried J. (1977): Was ist bei der Selektion landeskundlichen Wissens zu berücksichtigen? In: Jahrbuch Deutsch als Fremdsprache (IDS). Heidelberg: Groos, 2532.

Schmidt-Bernhardt, Angela / Stork, Antje / Adamczak-Krysztofowicz, Sylwia / Rybszleger, Paweł (2011): Deutsch-polnische Entdeckungen. Projektarbeit zur Förderung interkultureller Kompetenz in Schule und Studium. Theoretische Grundlagen und praktische Lernmaterialien. Marburg: Tectum.

Schweiger, Hannes / Hägi, Sara / Döll, Marion (2015): Landeskundliche und (kultur)reflexive Konzepte. Impulse für die Praxis. In: Fremdsprache Deutsch 52, 3-10.

Shafer, Naomi / Baumgartner, Martin (2017): Mehr als Länder- oder Landeskunde: Ansätze eines weitergedachten DACH-Prinzips. In: IDV-Magazin 92, 67-71.

Storch, Günther (1999): Deutsch als Fremdsprache. Eine Didaktik. Theoretische Grundlagen und praktische Unterrichtsgestaltung. München: Wilhelm Fink Verlag.

Studer, Thomas (2013): Braucht es ein neues DACH(L)? In: Demmig, Silvia / Hägi, Sara / Schweiger, Hannes (Hg.): DACH-Landeskunde: Theorie - Geschichte - Praxis. München: Iudicium, 67-96.

Tammenga-Helmantel, Marjon / Jentges, Sabine (2016): Universitäre Deutschlehrerausbildung in den Niederlanden: Grundlagen und Einflussfaktoren. In: ÖDaF-Mitteilungen 1, 73-85.

Tammenga-Helmantel, Marjon / Ciepielewska-Kaczmarek, Luiza / Jentges, Sabine (2017): Kultureller und sprachlicher Vielfalt auf der Spur: $\mathrm{DACH}(\mathrm{L})$-Praxis in polnischen und 
niederländischen DaF-Lehrwerken. In: IDV-Magazin: Das DACH-Prinzip 92/2017, $32-$ 38.

Tammenga-Helmantel, Marjon / Maijala, Minna / Donker, Eva (2017): Betekenisvol en uitdagend vreemdetalenonderwijs door talige en culturele variëteit. Een analyse van Nederlandse en Finse leergangen Duits. In: Levende Talen Magazine 104/1, 16-21.

Thaler, Engelbert (2011): Die Zukunft des Lehrwerks - Das Lehrwerk der Zukunft. In: FLuL - Fremdsprachen Lehren und Lernen 40 (2), 15-30.

Thomas, Alexander (1993): Kulturvergleichende Psychologie - Eine Einführung. Göttingen: Hogrefe.

Thornbury, Scott (2000): A Dogma for EFL. IATEFL Issues 153, 2.

Tomlinson, Brian (2012): Materials development for foreign language learning and teaching. In: Language Teaching 45, 143-179.

Tomlinson, Brian (2013): Materials development. In: Byram, Michael / Hu, Adelheid: Routledge Encyclopedia of Language Teaching and Learning. London, New York: Routledge, 450-456.

Vences, Ursula / Freudenstein, Reinhold (2002): Fremdsprachen lernen ohne Lehrwerk? Anmerkungen zu einer kontroversen These. In: Praxis 49, 295-299.

Vielau, Axel (2005): Lehrwerk, quo vadis? - Einflüsse auf die Lehrwerkentwicklung. In: Burwitz-Melzer, Eva / Solmecke, Gert (Hg.): Niemals zu früh und selten zu spät: Fremdsprachenunterricht in Schule und Erwachsenenbildung. Berlin: Cornelsen, 137147.

Wormer, Jörg (2004): Landeskunde - eine transkulturelle, vergleichende Wissenschaft. In: Zeitschrift für Interkulturellen Fremdsprachenunterricht 9 (3). Online: https://tujour nals.ulb.tu-darmstadt.de/index.php/zif/article/view/503/479 [Zugriff am 10.01.2019].

Wormer, Jörg (2006): Praxis - Forschung - Praxis: Elemente einer zukünftigen Landeskunde. In: Materialien Deutsch als Fremdsprache 76, 207-222.

Wrobel, Dieter / Ott, Christine (2018): Außerschulische Lernorte im Deutschunterricht: Anschlüsse - Zugänge - Kompetenzerwerb. Stuttgart: Klett.

Zeuner, Ulrich (2009): Landeskunde und interkulturelles Lernen. Eine Einführung. Dresden: Technische Universität.

Zeuner, Ulrich (2010): Interkulturelle Landeskunde. In: Krumm, Hans-Jürgen / Fandrych, Christian / Hufeisen, Britta / Riemer, Claudia (Hg.): Deutsch als Fremd- und Zweitsprache. Ein internationales Handbuch. Band 2. Berlin et al.: De Gruyter, 1472-1478.

Zeuner, Ulrich (2017): Interkulturelle Landeskunde - Diskursive Landeskunde: Ein Blick in zwei Lehrwerke. Vortragsskript: Leipzig 10.11.2017. Online: https://uzeuner.word press.com/2017/08/15/interkulturelle-landeskunde-diskursive-landeskunde-wo-sinddie-unterschiede/ [Zugriff am 10.01.2019].

Ziegler, Evelyn / Marten, Heiko F.: Linguistic Landscapes im deutschsprachigen Kontext: Forschungsperspektiven, Methoden und Anwendungsmöglichkeiten im Unterricht und Sprachmarketing. Reihe »Forum Angewandte Linguistik« (GAL). Berlin et al.: Peter Lang (in Vorbereitung). 


\section{Internetquellen}

https://www.goethe.de/resources/files/pdf37/Bro_Deutschlernerhebung_final2.pdf [Zugriff am 10.01.2019].

https://podstawaprogramowa.pl/Szkola-podstawowa-IV-VIII/Jezyk-obcy-nowozytny [Zugriff am 25.05.2019].

https://www.nuffic.nl/onderwerpen/starten-met-linq/ [Zugriff am 10.01.2019].

http://www.ru.nl/nachbarsprache/ [Zugriff am 10.01.2019].

https://ec.europa.eu/eurostat/statistics-explained/index.php?title=File:Proportion_of_pu pils_in_primary_education_learning_foreign_languages,_by_language,_2016_(\%25)_ ET18.png) [Zugriff am 10.09.2019]. 